\title{
Converting SMILES to Stacking Interaction Energies
}

\author{
Andrea N. Bootsma and Steven E. Wheeler* \\ Computational Quantum Chemistry, Department of Chemistry, University of Georgia, Athens, GA 30602 \\ E-mail: swheele2@uga.edu
}

Trends in Error for ESPmean

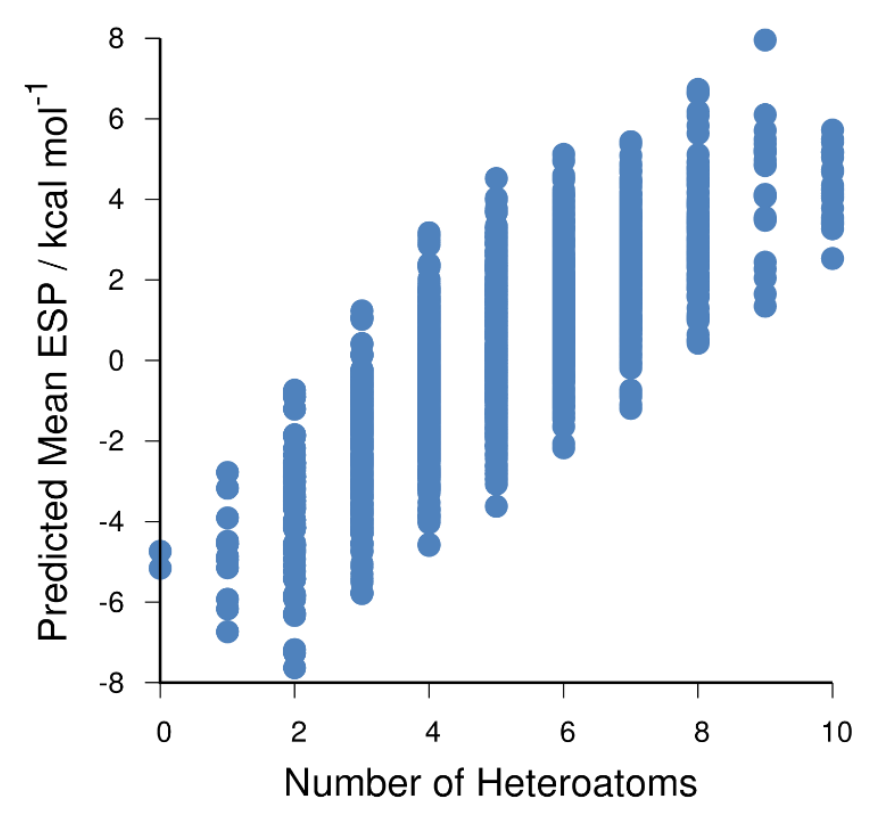

Figure S1. Trends in $\mathrm{ESP}_{\text {mean }}$ based on the number of heteroatoms in the heterocycle $(\mathrm{C}=\mathrm{O}$ is

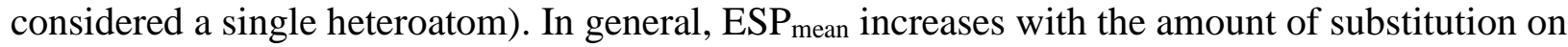
the ring, and a very large amount of substitution is required to achieve a high value of ESP mean. 


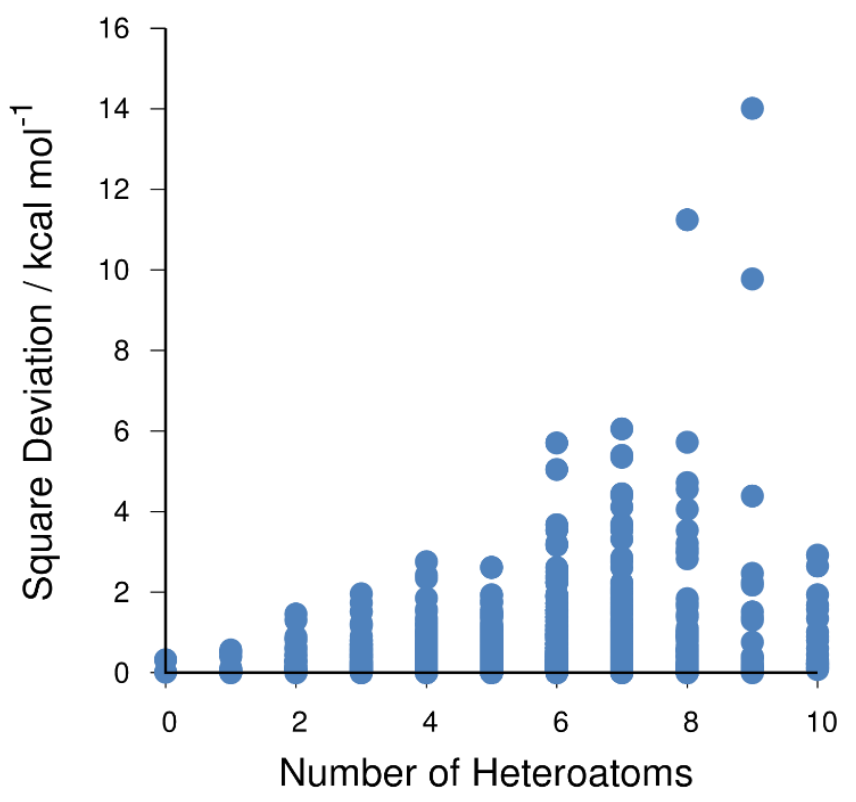

Figure S2. Trends in the error of equation 2 based on the number of heteroatoms in the heterocycle. In general, increasing amounts of substitution lead to increased errors. This is likely a result of a breakdown in the additivity assumed by equation (2) at high levels of substitution. This fact, combined with the trend seen in Figure S1, result in increased errors for large values of $\mathrm{ESP}_{\text {mean. }}$.

\section{Subset Trends}




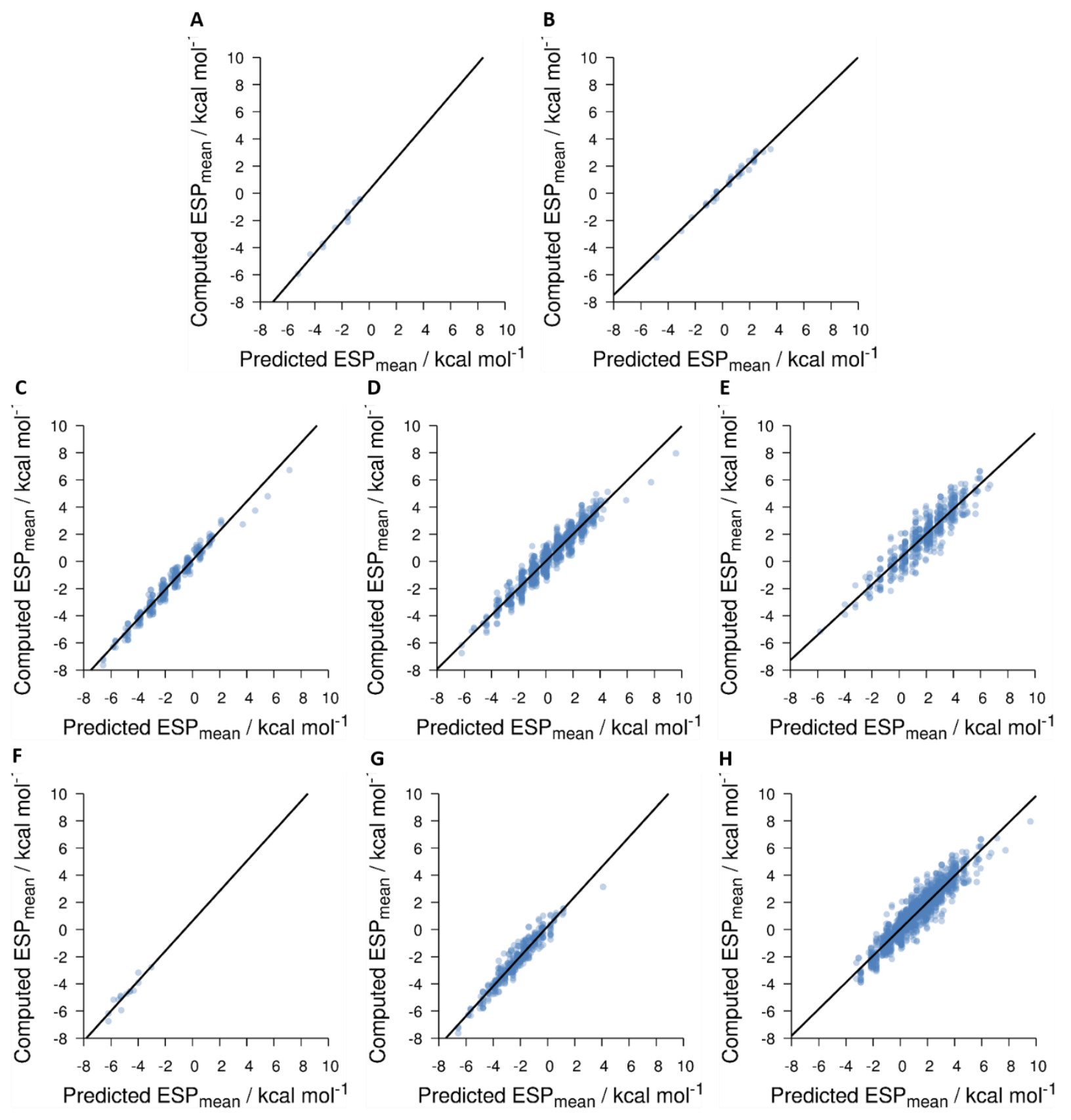

Figure S3. Scatter plots of DFT-computed ESP mean values vs values predicted using equation 2, split into subcategories: A) 5-membered monocycles; B) 6-membered monocycles; C) 5-5 bicycles; D) 5-6 bicycles; E) 6-6 bicycles; F) heterocycles with 0 or 1 heteroatoms; G) heterocycles with 2 or 3 heteroatoms; H) heterocycles with 4 or more heteroatoms. 

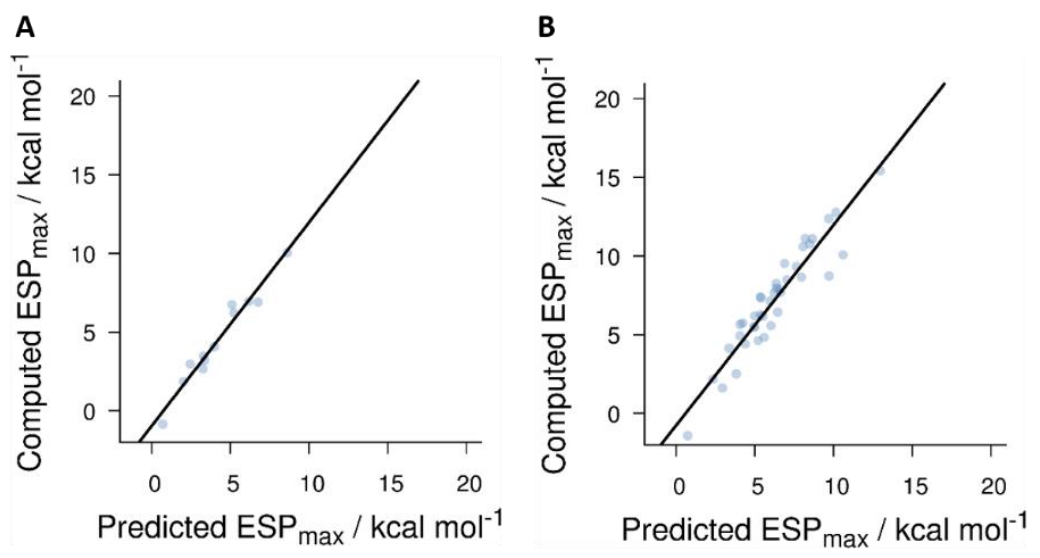

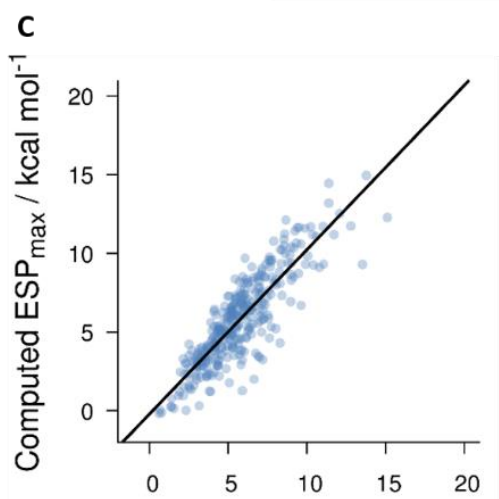

Predicted $\mathrm{ESP}_{\max } / \mathrm{kcal} \mathrm{mol}^{-1}$

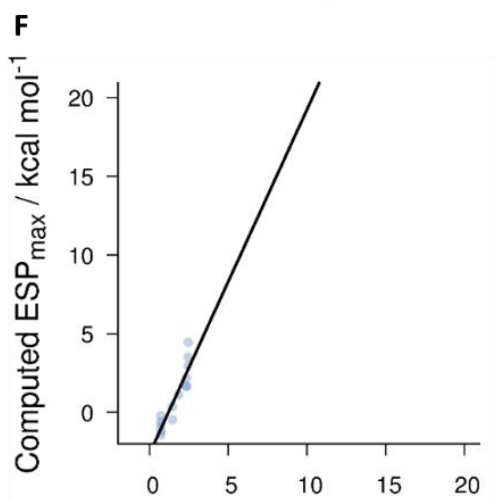

Predicted $\mathrm{ESP}_{\max } / \mathrm{kcal} \mathrm{mol}^{-1}$

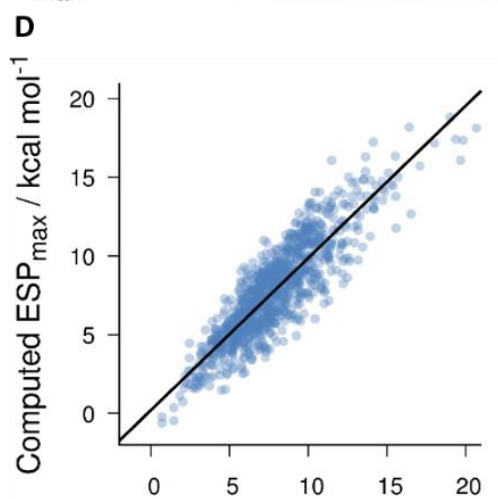

Predicted $\mathrm{ESP}_{\max } / \mathrm{kcal} \mathrm{mol}^{-1}$
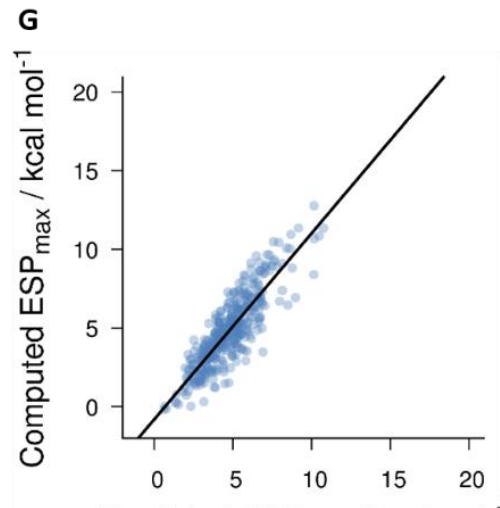

Predicted $\mathrm{ESP}_{\max } / \mathrm{kcal} \mathrm{mol}^{-1}$

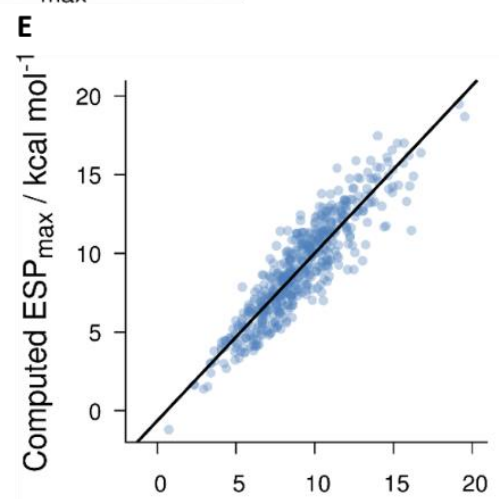

Predicted $\mathrm{ESP}_{\max } / \mathrm{kcal} \mathrm{mol}^{-1}$

H

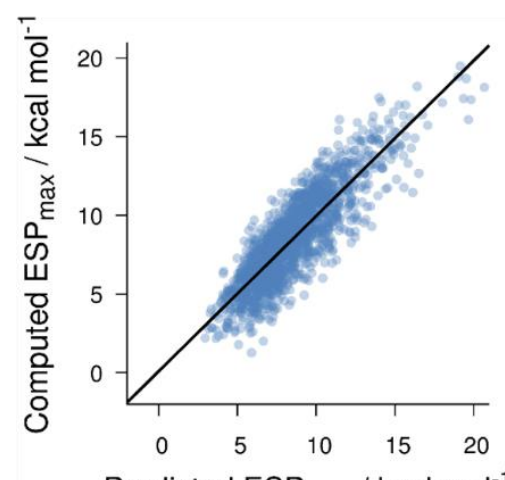

Predicted $\mathrm{ESP}_{\max } / \mathrm{kcal} \mathrm{mol}^{-1}$

Figure S4. Scatter plots of DFT-computed ESP $\max _{\max }$ values vs values predicted using equation (3), split into subcategories: A) 5-membered monocycles; B) 6-membered monocycles; C) 5-5 bicycles; D) 5-6 bicycles; E) 6-6 bicycles; F) heterocycles with 0 or 1 heteroatoms; G) heterocycles with 2 or 3 heteroatoms; H) heterocycles with 4 or more heteroatoms. 


\section{Fitting Other Descriptors}

$\mathrm{ESP}_{\text {range }}$ can be predicted using the same functional form as $\mathrm{ESP}_{\max }$ since it is similarly dependent on the number and arrangement of heteroatoms

$$
E S P_{\text {range }}^{\text {pred }}=e_{0}+\sum_{i}^{\text {hetatm }} e_{i}+\sum_{i<j}^{\text {hetatm }} \frac{\cos \left(\alpha_{i j}\right)}{R_{i j}} f_{i} f_{j}+g
$$

\begin{tabular}{lcc}
\hline Atom types & $\mathrm{e}_{\mathrm{i}}$ & $\mathrm{f}_{\mathrm{i}}$ \\
\hline $\mathrm{C}=\mathrm{O}$ & 3.08 & 2.97 \\
$\mathrm{~N}$ : (imino) & 0.65 & 2.12 \\
$\mathrm{O}$ & -0.29 & 0.37 \\
$\mathrm{~S}$ & -0.92 & 0.12 \\
$\mathrm{NH}$ (amino) & 3.00 & -1.67 \\
$\mathrm{~N}$ (ring fusion) & 1.67 & -0.60 \\
& $\mathrm{e}_{0}=11.22$ & $\mathrm{~g}=-2.15$ \\
\hline
\end{tabular}

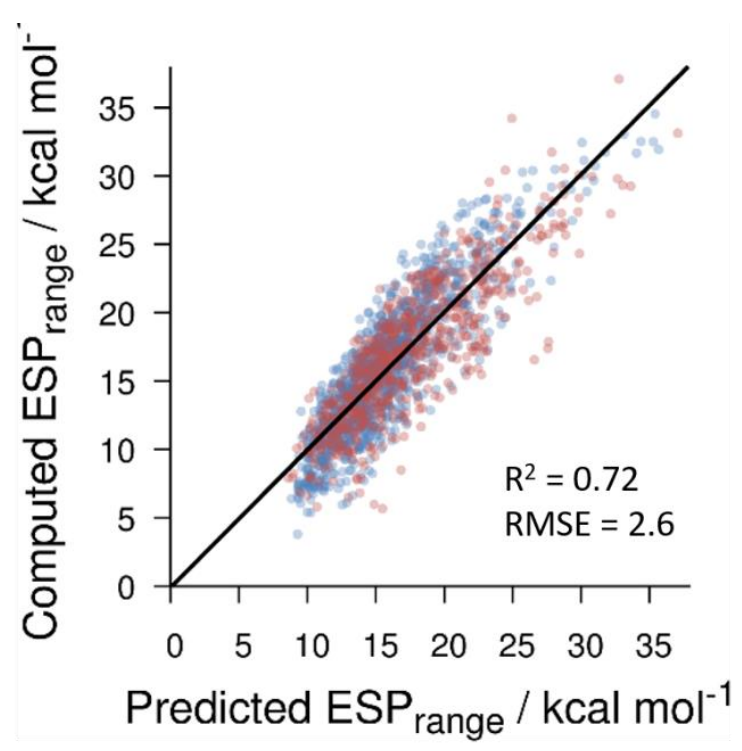

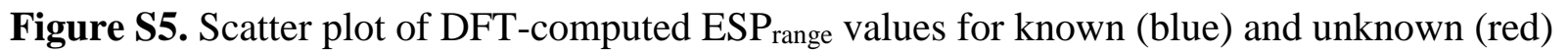
heterocycles vs. those predicted using equation (4) 
$F_{\text {mean }}$ can be predicted using a similar functional form that incorporates a constant shift to the geometry-based scaling factor.

$$
F_{\text {mean }}^{\text {pred }}=h_{0}+\sum_{i}^{\text {hetatm }} h_{i}+\sum_{i<j}^{\text {hetatm }}\left(\frac{\cos \left(\alpha_{i j}\right)}{R_{i j}}+j\right) k_{i} k_{j}+l
$$

\begin{tabular}{lcc}
\hline Atom types & $\mathrm{h}_{\mathrm{i}}$ & $\mathrm{k}_{\mathrm{i}}$ \\
\hline $\mathrm{C}=\mathrm{O}$ & -0.015 & 0.45 \\
$\mathrm{~N}$ ( (imino) & -0.038 & 0.37 \\
$\mathrm{O}$ & -0.004 & 0.13 \\
$\mathrm{~S}$ & -0.026 & 0.05 \\
$\mathrm{NH}$ (amino) & 0.17 & -0.25 \\
$\mathrm{~N}$ (ring fusion) & 0.099 & -0.12 \\
& $\mathrm{~h}_{0}=0.41$ & $1=-0.07$ \\
& $\mathrm{j}=0.29$ & \\
\hline
\end{tabular}

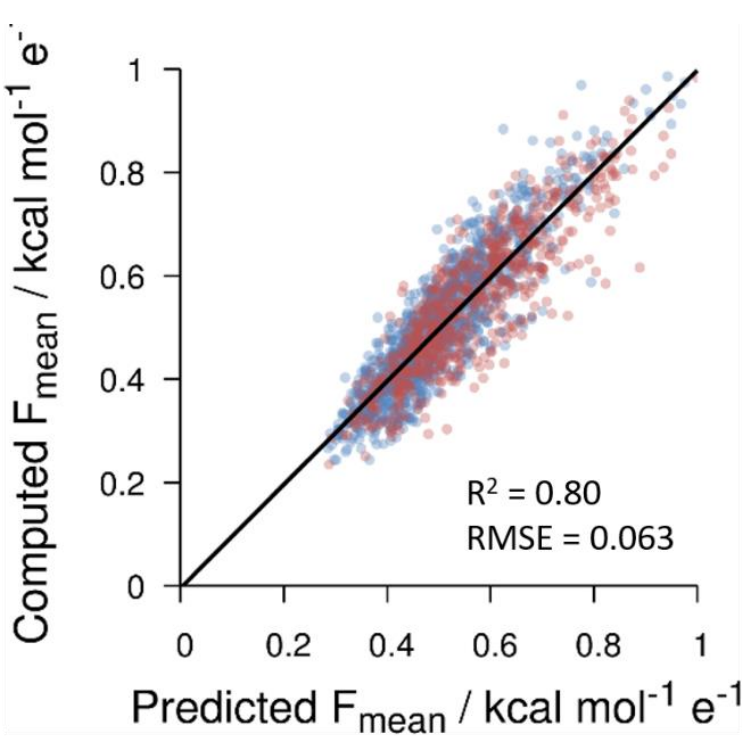

Figure S6. Scatter plot of DFT-computed $\mathrm{F}_{\text {mean }}$ values for known (blue) and unknown (red) heterocycles vs. those predicted using equation (5) 
Table S1. Values for distances and angles used in equation (3)-(5). Distances are based on a side length of 1 , angles in degrees<smiles>B1CCCC1</smiles><smiles>B1CCCOC1</smiles><smiles>B1COCC2BCC12</smiles><smiles></smiles><smiles>FC1CC[Te]CC1COP</smiles>

\begin{tabular}{|c|c|c|c|c|c|}
\hline \multicolumn{3}{|c|}{5 membered rings } & \multicolumn{3}{|c|}{6 membered rings } \\
\hline pair & distance & angle & pair & distance & angle \\
\hline A-B & 1 & 72 & A-B & 1 & 60 \\
\hline \multirow[t]{2}{*}{ A-C } & 1.62 & 144 & A-C & 1.73 & 120 \\
\hline & & & A-D & 2 & 180 \\
\hline \multicolumn{3}{|c|}{ 5-6 bicycles } & \multicolumn{3}{|c|}{ 6-6 bicycles } \\
\hline pair & distance & angle & pair & distance & angle \\
\hline A-C & 2.60 & 90 & A-D & 3.48 & 120 \\
\hline A-D & 3.30 & 150 & A-E & 3.62 & 180 \\
\hline B-C & 1.81 & 18 & B-C & 1.78 & 0 \\
\hline B-D & 2.69 & 78 & B-D & 2.68 & 60 \\
\hline B-E & 2.98 & 138 & B-E & 3.03 & 120 \\
\hline B-F & 2.57 & 162 & B-F & 2.69 & 180 \\
\hline
\end{tabular}


Alternate Energy Predictors

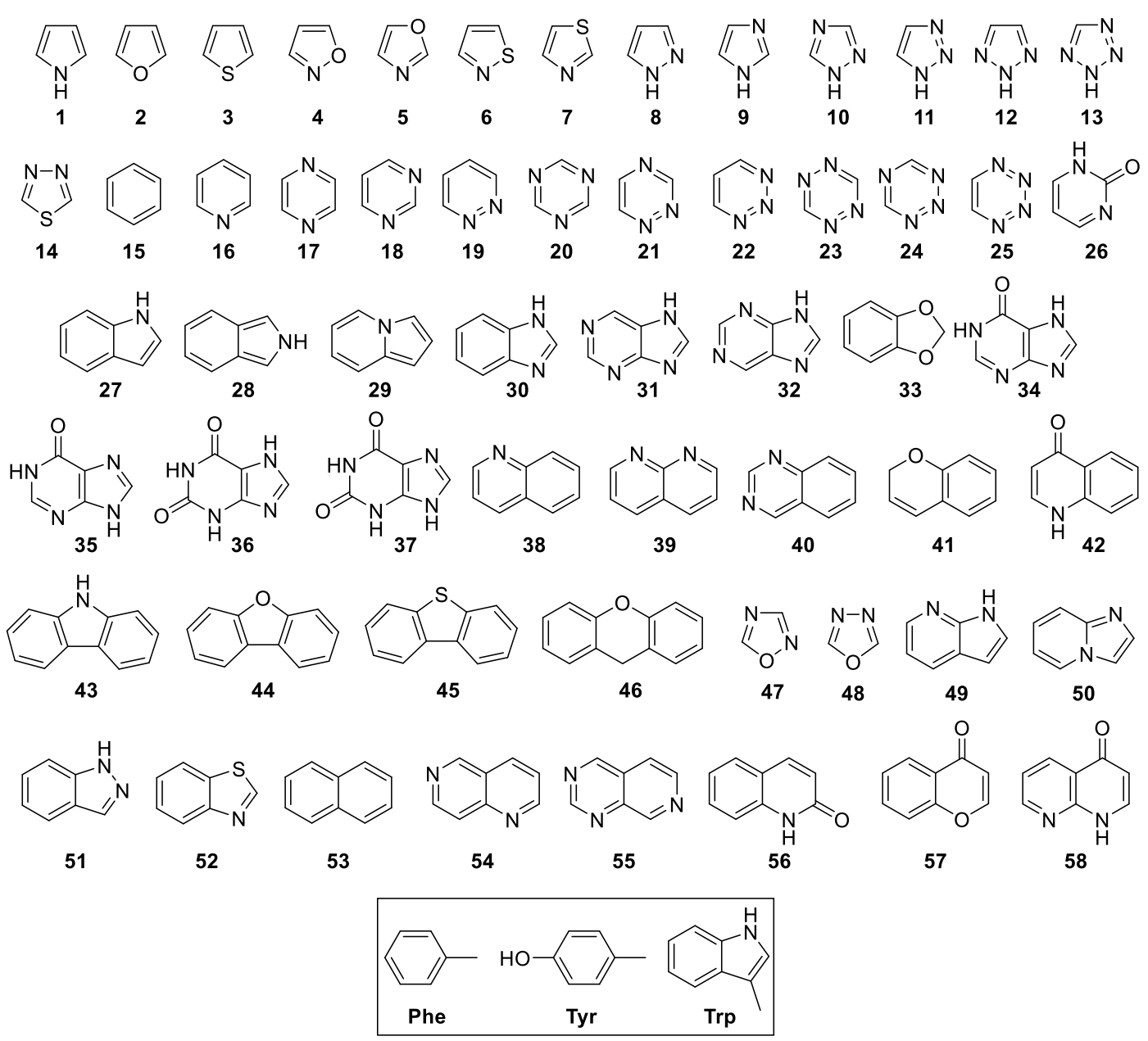

Chart S1. Heterocycles considered when comparing the use of DFT and SMILES-based descriptors for predicting stacking interaction energies. 
These atom connectivity based descriptors can be incorporated into other predictors of stacking energy.

$$
\Delta E_{\text {pred }}=N_{H A}^{A A}\left(-0.032 E S P_{\max }^{H e t}-0.087 N_{H A}^{H e t}\right)-1.46
$$

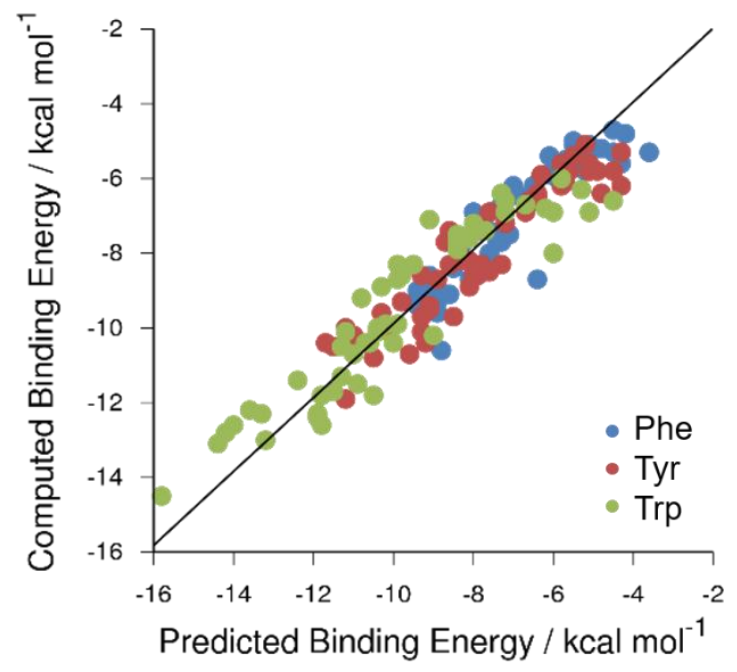

Figure S7. Scatter plot of computed DLPNO-CCSD(T)/cc-pVQZ interaction energies vs predicted interaction energies from equation (6) incorporating descriptors obtained from equation (3) for global minimum energy stacked dimers of heterocycles 1-42 and 47-58 with Phe, Tyr, and Trp side chains.

$$
\Delta E_{\text {pred }}=N_{H A}^{A A}\left(-0.036 E S P_{\text {mean }}^{\mathrm{Het}}-0.013 E S P_{\text {range }}^{\mathrm{Het}}-0.095 N_{H A}^{\mathrm{Het}}\right)-1.36
$$

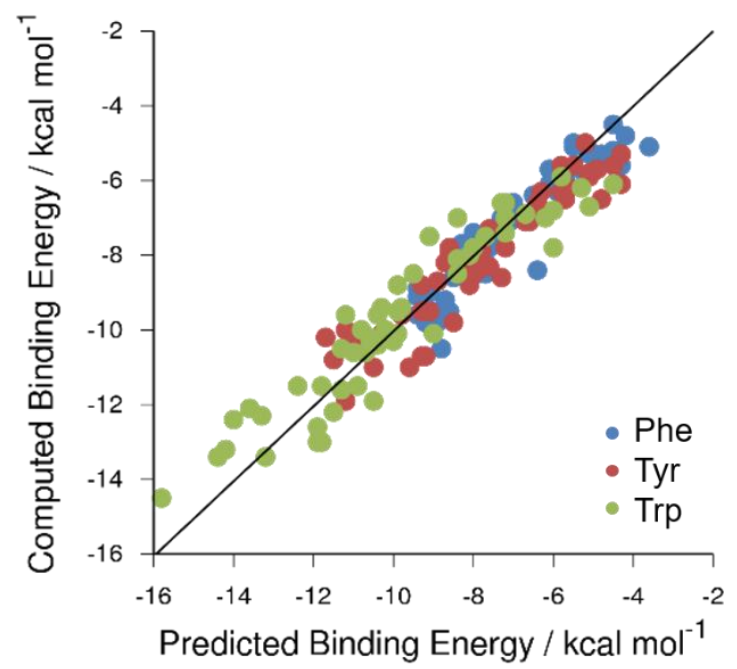

Figure S8. Scatter plot of computed DLPNO-CCSD(T)/cc-pVQZ interaction energies vs predicted interaction energies from equation (7) incorporating descriptors obtained from equations (2) and (4) for global minimum energy stacked dimers of heterocycles 1-42 and 47-58 with Phe, Tyr, and Trp side chains. 


$$
\Delta \mathrm{E}_{\text {pred }}=\mathrm{N}_{\mathrm{HA}}^{\mathrm{AA}}\left(-0.46 \mathrm{~F}_{\text {mean }}^{\mathrm{Het}}-0.042 \mathrm{ESP}_{\text {mean }}^{\mathrm{Het}}-0.098 \mathrm{~N}_{\mathrm{HA}}^{\mathrm{Het}}\right)-0.67
$$

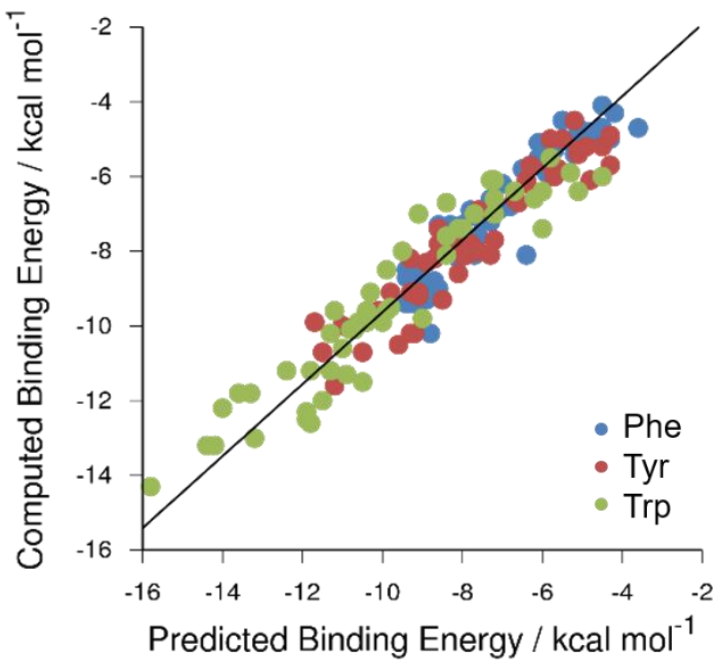

Figure S9. Scatter plot of computed DLPNO-CCSD(T)/cc-pVQZ interaction energies vs predicted interaction energies from equation (8) incorporating descriptors obtained from equations (2) and (5) for global minimum energy stacked dimers of heterocycles 1-42 and 47-58 with Phe, Tyr, and Trp side chains. 


\section{Checking Against Random Data}

Equations (2) and (3) were checked for overfitting by comparing both fits where the true ESP values were fit with random numbers of heteroatoms and heteroatom pairs, and where the true heteroatom and pair counts were used to fit random data. This was done using three different sets of random data

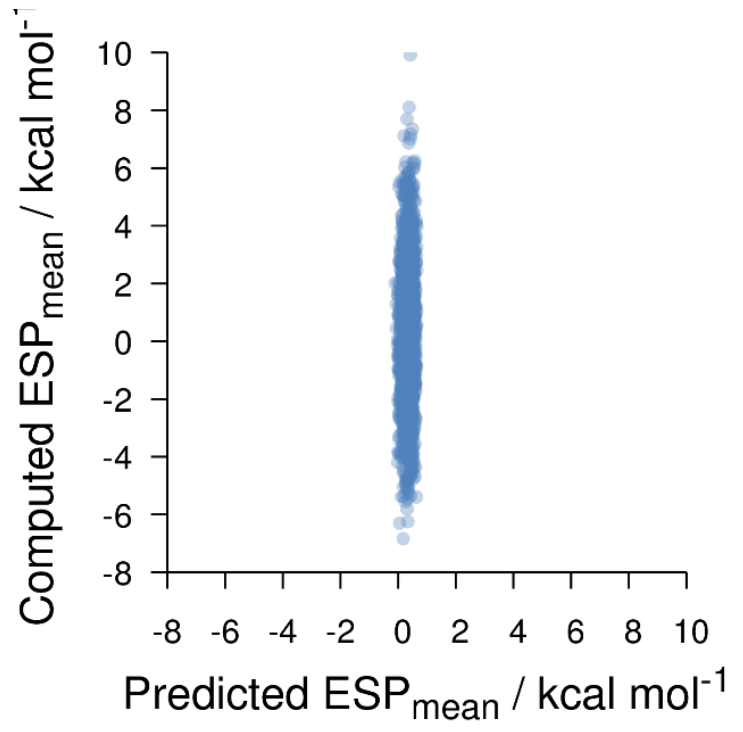

\begin{tabular}{ccc}
\hline & $\mathbf{R}^{\mathbf{2}}$ & $\mathbf{R M S E}$ \\
\hline Trial 1 & 0.00 & 2.35 \\
Trial 2 & 0.00 & 2.31 \\
Trial 3 & 0.00 & 2.37 \\
\hline
\end{tabular}

Figure S10. A representative example of random $\mathrm{ESP}_{\text {mean }}$ values predicted using real heteroatom counts, and the $\mathrm{R}^{2}$ and RMSE values for the three trials

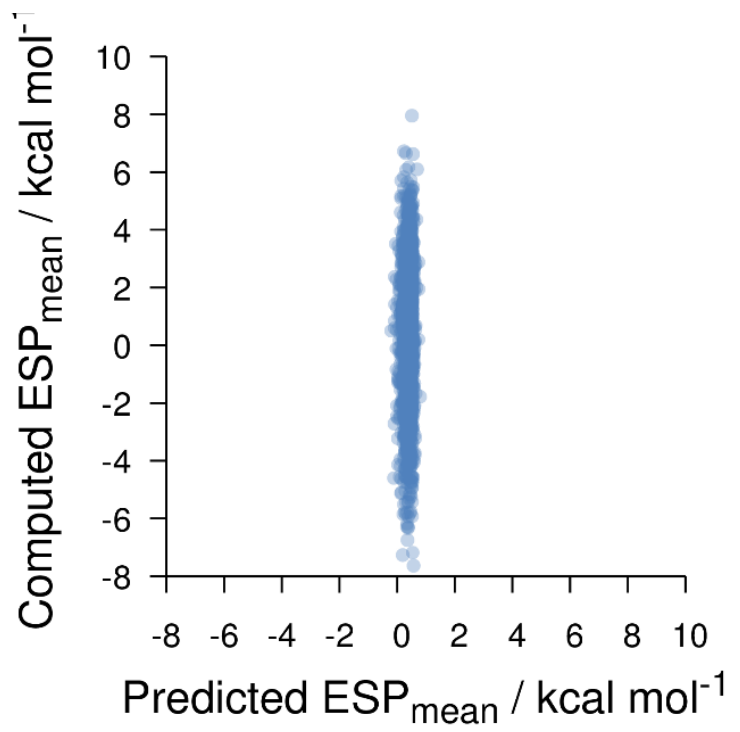

\begin{tabular}{ccc}
\hline & $\mathbf{R}^{\mathbf{2}}$ & $\mathbf{R M S E}$ \\
\hline Trial 1 & 0.00 & 2.33 \\
Trial 2 & 0.00 & 2.33 \\
Trial 3 & 0.00 & 2.33 \\
\hline
\end{tabular}

Figure S11. A representative example of real $\mathrm{ESP}_{\text {mean }}$ values predicted using random heteroatom counts, and the $\mathrm{R}^{2}$ and $\mathrm{RMSE}$ values for the three trials 


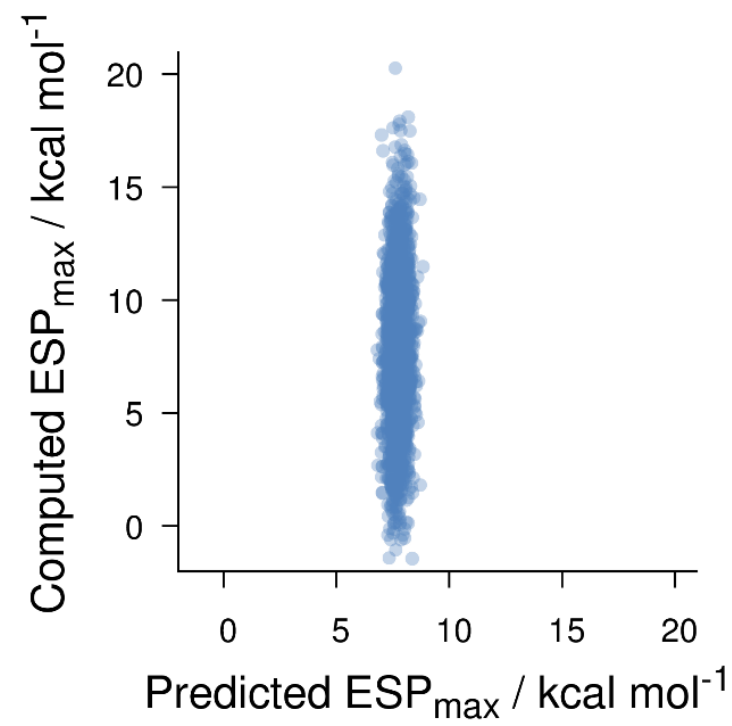

\begin{tabular}{ccc}
\hline & $\mathbf{R}^{\mathbf{2}}$ & $\mathbf{R M S E}$ \\
\hline Trial 1 & 0.01 & 3.33 \\
Trial 2 & 0.01 & 3.28 \\
Trial 3 & 0.01 & 3.25 \\
\hline
\end{tabular}

Figure S12. A representative example of random $\mathrm{ESP}_{\max }$ values predicted using real heteroatom counts, and the $\mathrm{R}^{2}$ and RMSE values for the three trials

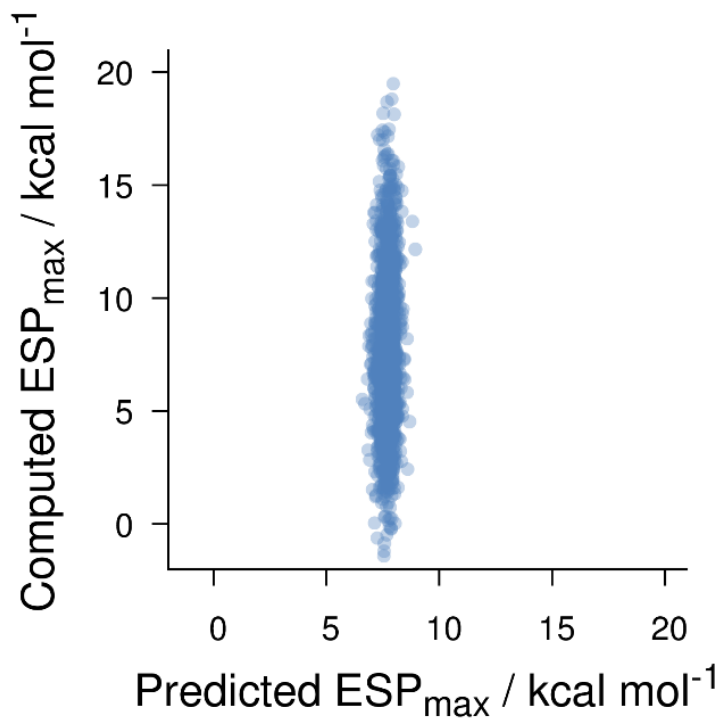

\begin{tabular}{ccc}
\hline & $\mathbf{R}^{\mathbf{2}}$ & $\mathbf{R M S E}$ \\
\hline Trial 1 & 0.01 & 3.27 \\
Trial 2 & 0.01 & 3.27 \\
Trial 3 & 0.00 & 3.27 \\
\hline
\end{tabular}

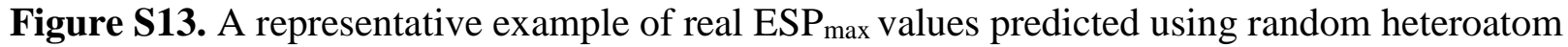
and heteroatom pair counts, and the $\mathrm{R}^{2}$ and RMSE values for the three trials 
Table S2. DLPNO-CCSD(T)/cc-pVQZ interaction energies for heterocycles stacked with amino acids and interaction energies predicted by multivariate fits (1), (6)-(7) using both QM descriptors and those obtained from SMILES via equations (2)-(5)

\begin{tabular}{|c|c|c|c|c|c|c|c|c|c|c|}
\hline \multirow{2}{*}{$\mathbf{A A}$} & \multirow{2}{*}{ Het } & \multicolumn{3}{|c|}{ Equation (1) } & \multicolumn{2}{|c|}{ Equation (6) } & \multicolumn{2}{|c|}{ Equation (7) } & \multicolumn{2}{|c|}{ Equation (8) } \\
\hline & & $\mathbf{E}_{\text {int }}$ & QM & SMILES & QM & SMILES & $\mathbf{Q M}$ & SMILES & $\mathbf{Q M}$ & SMILES \\
\hline Phe & 2 & -4.2 & -4.3 & -4.7 & -4.3 & -4.8 & -4.3 & -4.8 & -4.3 & -4.3 \\
\hline Phe & 3 & -4.5 & -4.2 & -4.4 & -4.3 & -4.7 & -4.2 & -4.5 & -4.3 & -4.1 \\
\hline Phe & 4 & -5.1 & -5.2 & -5.2 & -5.3 & -5.2 & -5.3 & -5.3 & -5.1 & -4.8 \\
\hline Phe & 5 & -5.1 & -5.1 & -5.1 & -5.0 & -5.1 & -5.0 & -5.2 & -4.8 & -4.7 \\
\hline Phe & 6 & -5.5 & -5.0 & -5.0 & -5.1 & -5.1 & -5.0 & -5.1 & -4.7 & -4.5 \\
\hline Phe & 7 & -5.5 & -4.9 & -4.9 & -4.9 & -5.0 & -4.8 & -5.0 & -4.6 & -4.5 \\
\hline Phe & 8 & -3.6 & -4.9 & -5.0 & -5.2 & -5.3 & -4.8 & -5.1 & -4.8 & -4.7 \\
\hline Phe & 10 & -4.3 & -5.8 & -5.5 & -6.0 & -5.6 & -5.5 & -5.6 & -5.3 & -5.0 \\
\hline Phe & 12 & -4.8 & -5.1 & -5.2 & -5.1 & -5.2 & -4.8 & -5.3 & -4.6 & -4.8 \\
\hline Phe & 13 & -5.2 & -6.0 & -5.9 & -6.0 & -5.8 & -5.9 & -5.9 & -5.6 & -5.4 \\
\hline Phe & 14 & -6.1 & -5.6 & -5.5 & -5.4 & -5.4 & -5.7 & -5.7 & -5.6 & -5.1 \\
\hline Phe & 15 & -4.5 & -4.7 & -5.0 & -4.8 & -5.3 & -4.8 & -5.2 & -5.0 & -4.7 \\
\hline Phe & 16 & -5.6 & -5.6 & -5.5 & -5.6 & -5.6 & -5.6 & -5.7 & -5.3 & -5.1 \\
\hline Phe & 17 & -6.0 & -5.8 & -5.9 & -5.5 & -5.8 & -5.6 & -6.0 & -5.5 & -5.4 \\
\hline Phe & 18 & -6.1 & -6.2 & -6.0 & -6.0 & -5.9 & -5.9 & -6.1 & -5.6 & -5.5 \\
\hline Phe & 19 & -6.5 & -6.4 & -6.2 & -6.3 & -6.2 & -6.6 & -6.4 & -6.3 & -5.8 \\
\hline Phe & 20 & -5.9 & -6.2 & -6.3 & -5.7 & -6.0 & -6.0 & -6.3 & -5.9 & -5.9 \\
\hline Phe & 21 & -7.0 & -6.8 & -6.5 & -6.5 & -6.2 & -6.7 & -6.6 & -6.5 & -6.2 \\
\hline Phe & 22 & -7.3 & -7.2 & -6.8 & -7.0 & -6.7 & -7.3 & -7.0 & -7.2 & -6.6 \\
\hline Phe & 23 & -6.8 & -7.0 & -6.9 & -6.4 & -6.5 & -6.7 & -7.0 & -7.1 & -6.8 \\
\hline Phe & 24 & -7.1 & -7.1 & -7.0 & -6.6 & -6.5 & -7.1 & -7.1 & -7.3 & -6.9 \\
\hline Phe & 25 & -8.0 & -7.8 & -7.3 & -7.5 & -6.9 & -7.7 & -7.4 & -7.9 & -7.3 \\
\hline Phe & 26 & -7.3 & -7.8 & -7.4 & -7.8 & -7.4 & -8.1 & -7.7 & -7.7 & -7.2 \\
\hline Phe & 27 & -7.3 & -7.2 & -7.1 & -7.7 & -7.5 & -7.2 & -7.1 & -7.1 & -6.9 \\
\hline Phe & 28 & -7.1 & -7.3 & -7.1 & -7.9 & -7.5 & -7.3 & -7.1 & -7.1 & -6.9 \\
\hline
\end{tabular}




\begin{tabular}{|c|c|c|c|c|c|c|c|c|c|c|}
\hline Phe & 29 & -7.6 & -7.0 & -7.8 & -7.2 & -8.0 & -7.1 & -7.8 & -7.0 & -7.6 \\
\hline Phe & 30 & -6.4 & -7.9 & -8.5 & -8.4 & -8.7 & -7.7 & -8.4 & -7.5 & -8.1 \\
\hline Phe & 31 & -9.4 & -9.3 & -8.9 & -9.5 & -9.0 & -9.2 & -8.9 & -8.8 & -8.5 \\
\hline Phe & 32 & -7.9 & -8.7 & -8.6 & -8.8 & -8.4 & -8.4 & -8.5 & -7.9 & -8.1 \\
\hline Phe & 33 & -7.6 & -7.9 & -7.4 & -8.0 & -7.4 & -7.7 & -7.4 & -7.4 & -7.1 \\
\hline Phe & 34 & -9.4 & -9.2 & -9.3 & -8.9 & -9.3 & -9.3 & -9.1 & -9.3 & -8.7 \\
\hline Phe & 35 & -9.2 & -9.3 & -9.8 & -9.2 & -9.6 & -9.6 & -9.8 & -9.3 & -9.4 \\
\hline Phe & 36 & -9.4 & -10.5 & -9.7 & -10.2 & -9.4 & -10.4 & -9.6 & -10.0 & -9.4 \\
\hline Phe & 37 & -8.8 & -11.4 & -10.5 & -11.5 & -10.6 & -11.4 & -10.5 & -11.0 & -10.2 \\
\hline Phe & 38 & -8.1 & -8.0 & -8.7 & -7.9 & -8.7 & -8.0 & -8.8 & -7.7 & -8.2 \\
\hline Phe & 39 & -8.9 & -8.6 & -9.4 & -8.5 & -9.3 & -8.9 & -9.5 & -8.5 & -9.0 \\
\hline Phe & 40 & -8.6 & -8.5 & -7.8 & -8.2 & -7.7 & -8.4 & -7.8 & -8.0 & -7.3 \\
\hline Phe & 41 & -7.7 & -8.2 & -8.5 & -8.3 & -8.5 & -8.1 & -8.5 & -7.9 & -8.1 \\
\hline Phe & 42 & -9.1 & -9.8 & -8.9 & -10.1 & -9.1 & -10.0 & -9.0 & -9.3 & -8.5 \\
\hline Phe & 48 & -5.7 & -5.7 & -5.7 & -5.6 & -5.5 & -5.9 & -5.9 & -6.0 & -5.3 \\
\hline Phe & 49 & -7.3 & -7.2 & -7.4 & -7.4 & -7.6 & -7.1 & -7.4 & -7.1 & -7.1 \\
\hline Phe & 50 & -8.3 & -7.7 & -7.7 & -7.8 & -7.8 & -7.8 & -7.7 & -7.6 & -7.3 \\
\hline Phe & 51 & -7.4 & -7.3 & -7.5 & -7.5 & -7.7 & -7.2 & -7.5 & -7.3 & -7.1 \\
\hline Phe & 52 & -7.8 & -7.2 & -7.4 & -7.1 & -7.4 & -7.1 & -7.5 & -7.1 & -6.9 \\
\hline Phe & 53 & -7.3 & -7.3 & -7.5 & -7.3 & -7.7 & -7.4 & -7.6 & -7.7 & -7.2 \\
\hline Phe & 54 & -8.5 & -8.6 & -8.5 & -8.4 & -8.4 & -8.5 & -8.6 & -8.2 & -8.0 \\
\hline Phe & 55 & -9.1 & -9.1 & -9.0 & -8.7 & -8.6 & -8.9 & -9.0 & -8.7 & -8.6 \\
\hline Phe & 56 & -8.7 & -9.1 & -9.1 & -8.8 & -9.1 & -9.5 & -9.2 & -9.2 & -8.8 \\
\hline Phe & 57 & -8.6 & -9.2 & -9.4 & -9.0 & -9.1 & -9.5 & -9.5 & -9.1 & -9.0 \\
\hline Phe & 58 & -8.9 & -9.6 & -9.7 & -9.6 & -9.6 & -9.8 & -9.8 & -9.3 & -9.3 \\
\hline Tyr & 2 & -4.3 & -4.6 & -5.1 & -4.8 & -5.3 & -4.7 & -5.3 & -4.8 & -4.9 \\
\hline Tyr & 3 & -5.2 & -4.5 & -4.8 & -4.7 & -5.1 & -4.6 & -5.0 & -4.8 & -4.5 \\
\hline Tyr & 4 & -5.1 & -5.7 & -5.7 & -5.8 & -5.8 & -5.9 & -5.9 & -5.8 & -5.4 \\
\hline Tyr & 5 & -5.1 & -5.6 & -5.6 & -5.5 & -5.6 & -5.6 & -5.8 & -5.3 & -5.3 \\
\hline Tyr & 6 & -5.8 & -5.5 & -5.5 & -5.6 & -5.6 & -5.5 & -5.6 & -5.3 & -5.0 \\
\hline Tyr & 7 & -5.5 & -5.4 & -5.4 & -5.4 & -5.4 & -5.3 & -5.6 & -5.2 & \\
\hline
\end{tabular}




\begin{tabular}{|c|c|c|c|c|c|c|c|c|c|c|}
\hline Tyr & 8 & -4.5 & -5.3 & -5.5 & -5.8 & -5.8 & -5.3 & -5.6 & -5.4 & -5.2 \\
\hline Tyr & 10 & -4.3 & -6.4 & -6.1 & -6.7 & -6.2 & -6.1 & -6.1 & -6.0 & -5.7 \\
\hline Tyr & 12 & -5.1 & -5.6 & -5.8 & -5.6 & -5.8 & -5.3 & -5.9 & -5.2 & -5.4 \\
\hline Tyr & 13 & -4.8 & -6.6 & -6.5 & -6.6 & -6.4 & -6.5 & -6.5 & -6.3 & -6.1 \\
\hline Tyr & 14 & -6.3 & -6.1 & -6.0 & -6.0 & -5.9 & -6.4 & -6.3 & -6.3 & -5.7 \\
\hline Tyr & 15 & -4.9 & -5.1 & -5.5 & -5.3 & -5.8 & -5.3 & -5.7 & -5.6 & -5.2 \\
\hline Tyr & 16 & -5.8 & -6.1 & -6.1 & -6.2 & -6.2 & -6.2 & -6.3 & -6.0 & -5.7 \\
\hline Tyr & 17 & -6.4 & -6.4 & -6.5 & -6.1 & -6.4 & -6.2 & -6.6 & -6.1 & -6.1 \\
\hline Tyr & 18 & -6.4 & -6.8 & -6.6 & -6.7 & -6.5 & -6.6 & -6.7 & -6.3 & -6.2 \\
\hline Tyr & 19 & -6.7 & -7.1 & -6.9 & -7.0 & -6.9 & -7.3 & -7.1 & -7.1 & -6.6 \\
\hline Tyr & 20 & -6.6 & -6.9 & -7.0 & -6.3 & -6.6 & -6.6 & -7.1 & -6.6 & -6.7 \\
\hline Tyr & 21 & -7.6 & -7.5 & -7.2 & -7.2 & -6.9 & -7.5 & -7.3 & -7.3 & -6.9 \\
\hline Tyr & 22 & -8.6 & -7.9 & -7.6 & -7.8 & -7.4 & -8.1 & -7.8 & -8.1 & -7.4 \\
\hline Tyr & 23 & -7.2 & -7.7 & -7.7 & -7.1 & -7.2 & -7.5 & -7.8 & -8.0 & -7.7 \\
\hline Tyr & 24 & -7.8 & -7.9 & -7.8 & -7.3 & -7.3 & -7.9 & -7.9 & -8.2 & -7.8 \\
\hline Tyr & 25 & -8.7 & -8.6 & -8.1 & -8.3 & -7.7 & -8.6 & -8.2 & -9.0 & -8.2 \\
\hline Tyr & 26 & -7.3 & -8.6 & -8.2 & -8.7 & -8.3 & -9.0 & -8.6 & -8.7 & -8.1 \\
\hline Tyr & 27 & -8.6 & -8.0 & -7.9 & -8.6 & -8.3 & -8.0 & -7.9 & -8.0 & -7.8 \\
\hline Tyr & 28 & -7.8 & -8.1 & -7.9 & -8.9 & -8.3 & -8.1 & -7.9 & -8.0 & -7.8 \\
\hline Tyr & 29 & -8.1 & -7.8 & -8.7 & -8.0 & -8.9 & -7.9 & -8.8 & -7.9 & -8.6 \\
\hline Tyr & 30 & -9.3 & -8.8 & -9.4 & -9.4 & -9.7 & -8.7 & -9.5 & -8.5 & -9.1 \\
\hline Tyr & 31 & -11.2 & -10.4 & -10.0 & -10.6 & -10.0 & -10.3 & -10.0 & -9.9 & -9.6 \\
\hline Tyr & 32 & -9.1 & -9.8 & -9.6 & -9.8 & -9.4 & -9.4 & -9.5 & -8.9 & -9.1 \\
\hline Tyr & 33 & -8.3 & -8.8 & -8.2 & -8.9 & -8.2 & -8.6 & -8.3 & -8.4 & -8.0 \\
\hline Tyr & 34 & -11.7 & -10.3 & -10.4 & -10.0 & -10.4 & -10.5 & -10.2 & -10.6 & -9.9 \\
\hline Tyr & 35 & -10.5 & -10.4 & -10.9 & -10.3 & -10.8 & -10.8 & -11.0 & -10.6 & -10.7 \\
\hline Tyr & 36 & -11.5 & -11.8 & -10.8 & -11.4 & -10.5 & -11.7 & -10.8 & -11.3 & -10 . \\
\hline Tyr & 37 & -11.2 & -12.8 & -11.8 & -12.9 & -11.9 & -12.8 & -11.9 & -12.5 & -11 . \\
\hline Tyr & 38 & -8.5 & -8.9 & -9.7 & -8.8 & -9.7 & -8.9 & -9.8 & -8.8 & -9.3 \\
\hline Tyr & 39 & -9.2 & -9.6 & -10.5 & -9.5 & -10.4 & -10.0 & -10.7 & -9.6 & -10 . \\
\hline Tyr & 40 & -9.3 & -9.5 & -8.7 & -9.2 & -8.6 & -9.4 & -8.8 & -9.1 & \\
\hline
\end{tabular}




\begin{tabular}{|c|c|c|c|c|c|c|c|c|c|c|}
\hline Tyr & 41 & -9.1 & -9.1 & -9.4 & -9.3 & -9.5 & -9.1 & -9.5 & -8.9 & -9.2 \\
\hline Tyr & 42 & -10.1 & -11.0 & -9.9 & -11.3 & -10.1 & -11.2 & -10.1 & -10.5 & -9.6 \\
\hline Tyr & 47 & -5.6 & -6.3 & -6.1 & -6.1 & -5.8 & -6.2 & -6.2 & -6.1 & -5.8 \\
\hline Tyr & 48 & -5.7 & -6.3 & -6.3 & -6.2 & -6.1 & -6.6 & -6.5 & -6.8 & -6.0 \\
\hline Tyr & 49 & -7.6 & -8.0 & -8.3 & -8.2 & -8.5 & -7.9 & -8.3 & -8.0 & -8.0 \\
\hline Tyr & 50 & -8.9 & -8.5 & -8.6 & -8.7 & -8.7 & -8.8 & -8.7 & -8.6 & -8.3 \\
\hline Tyr & 51 & -7.9 & -8.2 & -8.3 & -8.3 & -8.6 & -8.1 & -8.4 & -8.2 & -8.1 \\
\hline Tyr & 52 & -8.1 & -8.0 & -8.3 & -7.9 & -8.2 & -8.0 & -8.4 & -8.0 & -7.8 \\
\hline Tyr & 53 & -8.0 & -8.1 & -8.4 & -8.1 & -8.6 & -8.3 & -8.5 & -8.7 & -8.1 \\
\hline Tyr & 54 & -9.8 & -9.6 & -9.5 & -9.4 & -9.3 & -9.5 & -9.6 & -9.3 & -9.1 \\
\hline Tyr & 55 & -10.3 & -10.1 & -10.1 & -9.8 & -9.6 & -10.0 & -10.1 & -9.8 & -9.7 \\
\hline Tyr & 56 & -11.0 & -10.2 & -10.2 & -9.9 & -10.2 & -10.6 & -10.4 & -10.4 & -10.0 \\
\hline Tyr & 57 & -9.3 & -10.3 & -10.5 & -10.1 & -10.1 & -10.6 & -10.7 & -10.3 & -10.2 \\
\hline Tyr & 58 & -9.6 & -10.8 & -10.9 & -10.8 & -10.7 & -11.1 & -11.0 & -10.5 & -10.5 \\
\hline Trp & 1 & -4.5 & -5.8 & -5.8 & -6.8 & -6.6 & -5.9 & -6.1 & -6.3 & -6.0 \\
\hline Trp & 2 & -5.3 & -5.4 & -5.9 & -5.6 & -6.3 & -5.5 & -6.2 & -5.9 & -5.9 \\
\hline Trp & 3 & -5.8 & -5.2 & -5.6 & -5.5 & -6.0 & -5.4 & -5.9 & -5.8 & -5.5 \\
\hline Trp & 4 & -7.2 & -6.8 & -6.7 & -6.9 & -6.9 & -7.0 & -7.0 & -7.0 & -6.6 \\
\hline Trp & 5 & -6.7 & -6.5 & -6.6 & -6.5 & -6.7 & -6.6 & -6.9 & -6.5 & -6.4 \\
\hline Trp & 6 & -7.2 & -6.5 & -6.4 & -6.7 & -6.6 & -6.5 & -6.6 & -6.5 & -6.1 \\
\hline Trp & 7 & -7.3 & -6.3 & -6.3 & -6.4 & -6.4 & -6.3 & -6.6 & -6.3 & -6.1 \\
\hline Trp & 8 & -5.1 & -6.3 & -6.4 & -6.8 & -6.9 & -6.3 & -6.7 & -6.5 & -6.4 \\
\hline Trp & 9 & -8.4 & -7.0 & -6.8 & -7.8 & -7.5 & -7.1 & -7.0 & -7.3 & -6.7 \\
\hline Trp & 11 & -6.0 & -7.5 & -7.5 & -8.0 & -8.0 & -7.8 & -7.8 & -8.0 & -7.4 \\
\hline Trp & 12 & -6.2 & -6.5 & -6.8 & -6.7 & -6.8 & -6.3 & -7.0 & -6.3 & -6.6 \\
\hline Trp & 14 & -9.1 & -7.2 & -7.1 & -7.1 & -7.1 & -7.6 & -7.5 & -7.8 & -7.0 \\
\hline Trp & 15 & -6.0 & -6.0 & -6.5 & -6.2 & -6.9 & -6.3 & -6.8 & -6.9 & -6.4 \\
\hline Trp & 16 & -7.7 & -7.2 & -7.2 & -7.4 & -7.4 & -7.4 & -7.5 & -7.3 & -7.0 \\
\hline Trp & 17 & -8.1 & -7.5 & -7.7 & -7.2 & -7.6 & -7.4 & -8.0 & -7.5 & -7.4 \\
\hline Trp & 18 & -8.4 & -8.1 & -7.8 & -8.0 & -7.7 & -7.9 & -8.1 & -7.7 & -7.6 \\
\hline Trp & 19 & -9.5 & -8.4 & -8.2 & -8.4 & -8.3 & -8.8 & -8.5 & -8.8 & -8.0 \\
\hline
\end{tabular}




\begin{tabular}{|c|c|c|c|c|c|c|c|c|c|c|}
\hline Trp & 20 & -8.4 & -8.2 & -8.3 & -7.5 & -7.9 & -7.9 & -8.5 & -8.1 & -8.1 \\
\hline Trp & 21 & -9.9 & -9.0 & -8.6 & -8.7 & -8.3 & -9.0 & -8.8 & -9.0 & -8.5 \\
\hline $\operatorname{Trp}$ & 22 & -10.3 & -9.5 & -9.0 & -9.4 & -8.9 & -9.8 & -9.4 & -10.0 & -9.1 \\
\hline Trp & 23 & -9.8 & -9.2 & -9.2 & -8.5 & -8.6 & -9.0 & -9.4 & -9.8 & -9.5 \\
\hline Trp & 24 & -9.9 & -9.4 & -9.3 & -8.7 & -8.7 & -9.6 & -9.5 & -10.1 & -9.6 \\
\hline Trp & 25 & -10.8 & -10.4 & -9.7 & -10.1 & -9.2 & -10.3 & -10.0 & -11.1 & -10.1 \\
\hline $\operatorname{Trp}$ & 26 & -10.4 & -10.4 & -9.9 & -10.5 & -10.0 & -11.0 & -10.4 & -10.7 & -9.9 \\
\hline $\operatorname{Trp}$ & 27 & -11.2 & -9.6 & -9.4 & -10.4 & -10.1 & -9.7 & -9.6 & -9.8 & -9.6 \\
\hline $\operatorname{Trp}$ & 28 & -10.4 & -9.7 & -9.4 & -10.7 & -10.1 & -9.8 & -9.6 & -9.8 & -9.6 \\
\hline Trp & 29 & -11.0 & -9.3 & -10.4 & -9.6 & -10.7 & -9.5 & -10.6 & -9.7 & -10.6 \\
\hline $\operatorname{Trp}$ & 30 & -11.8 & -10.6 & -11.4 & -11.3 & -11.8 & -10.5 & -11.5 & -10.4 & -11.2 \\
\hline $\operatorname{Trp}$ & 31 & -13.6 & -12.5 & -12.1 & -12.9 & -12.2 & -12.5 & -12.1 & -12.2 & -11.8 \\
\hline Trp & 32 & -12.4 & -11.8 & -11.5 & -11.9 & -11.4 & -11.5 & -11.5 & -11.0 & -11.2 \\
\hline $\operatorname{Trp}$ & 33 & -10.2 & -10.6 & -9.9 & -10.8 & -9.9 & -10.4 & -10.0 & -10.3 & -9.8 \\
\hline Trp & 34 & -14.0 & -12.4 & -12.5 & -12.1 & -12.6 & -12.7 & -12.4 & -13.1 & -12.2 \\
\hline $\operatorname{Trp}$ & 35 & -14.4 & -12.5 & -13.2 & -12.5 & -13.1 & -13.1 & -13.4 & -13.1 & -13.2 \\
\hline $\operatorname{Trp}$ & 36 & -14.2 & -14.3 & -13.1 & -13.9 & -12.8 & -14.3 & -13.2 & -14.0 & -13.2 \\
\hline Trp & 37 & -15.8 & -15.6 & -14.3 & -15.8 & -14.5 & -15.7 & -14.5 & -15.4 & -14.3 \\
\hline $\operatorname{Trp}$ & 38 & -10.5 & -10.7 & -11.8 & -10.7 & -11.8 & -10.8 & -11.9 & -10.8 & -11.5 \\
\hline $\operatorname{Trp}$ & 39 & -11.8 & -11.6 & -12.8 & -11.5 & -12.6 & -12.1 & -13.0 & -11.9 & -12.6 \\
\hline Trp & 40 & -10.7 & -11.4 & -10.5 & -11.1 & -10.4 & -11.4 & -10.6 & -11.2 & -10.1 \\
\hline Trp & 41 & -10.9 & -11.0 & -11.4 & -11.2 & -11.5 & -11.0 & -11.5 & -11.0 & -11.3 \\
\hline Trp & 42 & -13.3 & -13.3 & -12.0 & -13.8 & -12.3 & -13.6 & -12.3 & -13.0 & -11.8 \\
\hline Trp & 47 & -7.2 & -7.4 & -7.2 & -7.3 & -6.9 & -7.4 & -7.4 & -7.5 & -7.0 \\
\hline $\operatorname{Trp}$ & 48 & -8.0 & -7.5 & -7.4 & -7.4 & -7.2 & -7.9 & -7.8 & -8.3 & -7.4 \\
\hline Trp & 49 & -9.0 & -9.6 & -9.9 & -9.9 & -10.2 & -9.6 & -10.1 & -9.9 & -9.8 \\
\hline Trp & 50 & -11.3 & -10.3 & -10.3 & -10.5 & -10.5 & -10.6 & -10.5 & -10.6 & -10.2 \\
\hline Trp & 51 & -10.6 & -9.8 & -10.0 & -10.0 & -10.4 & -9.7 & -10.2 & -10.1 & -9.9 \\
\hline Trp & 52 & -9.9 & -9.6 & -9.9 & -9.5 & -9.9 & -9.6 & -10.1 & -9.9 & -9.6 \\
\hline Trp & 53 & -10.0 & -9.7 & -10.1 & -9.8 & -10.4 & -10.0 & -10.3 & -10.7 & -9.9 \\
\hline $\operatorname{Trp}$ & 54 & -11.3 & -11.5 & -11.5 & -11.4 & -11.3 & -11.5 & -11.6 & -11.4 & -11.2 \\
\hline
\end{tabular}




\begin{tabular}{ccccccccccc} 
Trp & $\mathbf{5 5}$ & -11.5 & -12.3 & -12.2 & -11.8 & -11.7 & -12.1 & -12.2 & -12.1 & -12.0 \\
Trp & $\mathbf{5 6}$ & -11.9 & -12.3 & -12.4 & -12.0 & -12.4 & -13.0 & -12.6 & -12.8 & -12.3 \\
Trp & $\mathbf{5 7}$ & -11.9 & -12.5 & -12.7 & -12.3 & -12.3 & -12.9 & -13.0 & -12.7 & -12.5 \\
Trp & $\mathbf{5 8}$ & -13.2 & -13.1 & -13.2 & -13.1 & -13.0 & -13.5 & -13.4 & -13.0 & -13.0 \\
\hline $\mathbf{R}^{2}$ & -- & 0.91 & 0.90 & 0.89 & 0.89 & 0.91 & 0.90 & 0.92 & 0.91 \\
$\boldsymbol{\rho}^{2}$ & -- & 0.91 & 0.90 & 0.89 & 0.88 & 0.92 & 0.91 & 0.92 & 0.91 \\
$\boldsymbol{\tau}$ & -- & 0.83 & 0.82 & 0.81 & 0.80 & 0.84 & 0.82 & 0.84 & 0.82 \\
RMSE & -- & 0.7 & 0.8 & 0.8 & 0.8 & 0.7 & 0.8 & 0.7 & 0.8 \\
\hline
\end{tabular}


Table S3. DFT-computed (Comp.) and Predicted (Pred.; from equations 2-5) values of ESP mean, $_{\text {ESP }}$ max $, \mathrm{ESP}_{\text {range }}, \mathrm{F}_{\text {mean }}$ for the set of 1854 heterocycles. IDs are from the VEHICLe database. This data is also available as a CSV (see the ACS Publications website).

\begin{tabular}{|c|c|c|c|c|c|c|c|c|c|}
\hline \multirow{2}{*}{ ID } & \multirow{2}{*}{ SMILES } & \multicolumn{2}{|c|}{ ESP $_{\text {mean }}$} & \multicolumn{2}{|c|}{$\mathbf{E S P}_{\max }$} & \multicolumn{2}{|c|}{ ESPrange } & \multicolumn{2}{|c|}{$F_{\text {mean }}$} \\
\hline & & Comp. & Pred. & Comp. & Pred. & Comp. & Pred. & Comp. & Pred. \\
\hline S1 & $\mathrm{c} 1 \mathrm{ccc}[\mathrm{nH}] 1$ & -5.93 & -5.23 & 2.98 & 2.46 & 14.71 & 14.22 & 0.35 & 0.35 \\
\hline S10 & $\mathrm{c} 1 \mathrm{cnn}[\mathrm{nH}] 1$ & -1.80 & -1.57 & 6.91 & 6.77 & 17.80 & 17.59 & 0.40 & 0.42 \\
\hline S10016 & $\mathrm{O}=\mathrm{c} 1[\mathrm{nH}] \mathrm{ncc} 2 \mathrm{c} 1 \mathrm{c}[\mathrm{nH}] \mathrm{c} 2$ & -2.63 & -1.76 & 9.04 & 5.73 & 20.96 & 15.08 & 0.64 & 0.62 \\
\hline S10017 & $\mathrm{O}=\mathrm{c} 1[\mathrm{nH}] \mathrm{ncc} 2 \mathrm{c} 1 \operatorname{coc} 2$ & -0.45 & -0.16 & 5.33 & 4.83 & 13.98 & 11.55 & 0.70 & 0.68 \\
\hline S10018 & $\mathrm{O}=\mathrm{c} 1[\mathrm{nH}] \mathrm{ncc} 2 \mathrm{c} 1 \mathrm{csc} 2$ & -0.99 & -0.85 & 4.96 & 4.07 & 13.95 & 10.95 & 0.49 & 0.49 \\
\hline S10019 & $\mathrm{O}=\mathrm{c} 1[\mathrm{nH}] \mathrm{ncc} 2 \mathrm{c} 1 \mathrm{c}[\mathrm{nH}] \mathrm{n} 2$ & -0.89 & 0.07 & 8.53 & 6.54 & 17.17 & 13.53 & 0.45 & 0.43 \\
\hline S10020 & $\mathrm{O}=\mathrm{c} 1[\mathrm{nH}] \mathrm{ncc} 2 \mathrm{c} 1 \mathrm{n}[\mathrm{nH}] \mathrm{c} 2$ & -1.02 & 0.07 & 9.34 & 7.14 & 21.98 & 16.55 & 0.50 & 0.54 \\
\hline S10021 & $\mathrm{O}=\mathrm{c} 1[\mathrm{nH}] \mathrm{ncc} 2 \mathrm{c} 1 \mathrm{con} 2$ & 1.61 & 1.67 & 4.42 & 6.59 & 9.38 & 11.34 & 0.35 & 0.36 \\
\hline S10022 & $\mathrm{O}=\mathrm{c} 1[\mathrm{nH}] \mathrm{ncc} 2 \mathrm{c} 1 \mathrm{noc} 2$ & 1.48 & 1.67 & 8.97 & 7.19 & 18.56 & 14.36 & 0.40 & 0.38 \\
\hline S10025 & $\mathrm{O}=\mathrm{c} 1[\mathrm{nH}] \mathrm{ncc} 2 \mathrm{c} 1 \mathrm{n}[\mathrm{nH}] \mathrm{n} 2$ & 0.77 & 1.90 & 7.47 & 6.93 & 16.60 & 12.76 & 0.42 & 0.40 \\
\hline S10026 & $\mathrm{O}=\mathrm{c} 1[\mathrm{nH}] \mathrm{ncc} 2 \mathrm{c} 1 \mathrm{non} 2$ & 3.57 & 3.50 & 8.67 & 7.93 & 14.07 & 11.90 & 0.41 & 0.45 \\
\hline S10027 & $\mathrm{O}=\mathrm{c} 1[\mathrm{nH}] \mathrm{ncc} 2 \mathrm{c} 1 \mathrm{nsn} 2$ & 2.23 & 2.81 & 6.00 & 7.05 & 13.70 & 10.98 & 0.63 & 0.63 \\
\hline S10028 & $=\mathrm{c} 1[\mathrm{nH}] \mathrm{cc} 2 \mathrm{c}(\mathrm{c} 1) \mathrm{cn}[\mathrm{nH}] \mathrm{c} 2$ & 1.63 & 1.22 & 7.33 & 7.34 & 14.25 & 14.14 & 0.39 & 0.39 \\
\hline S10029 & $\mathrm{O}=\mathrm{c} 1[\mathrm{nH}] \mathrm{cc} 2 \mathrm{c}(\mathrm{c} 1) \mathrm{c}(=\mathrm{O})[\mathrm{nH}] \mathrm{nc} 2$ & 1.44 & 1.22 & 11.42 & 7.82 & 19.71 & 16.66 & 0.66 & 0.66 \\
\hline S10034 & $\mathrm{O}=\mathrm{c} 1[\mathrm{nH}] \mathrm{ncc} 2 \mathrm{c} 1 \mathrm{c}(=\mathrm{O})[\mathrm{nH}] \mathrm{nc} 2$ & 1.98 & 3.05 & 8.61 & 7.72 & 20.05 & & 0.41 & 0.41 \\
\hline S10035 & $\mathrm{O}=\mathrm{c} 1[\mathrm{nH}] \mathrm{ncc} 2 \mathrm{c} 1 \mathrm{cn}[\mathrm{nH}] \mathrm{c} 2=\mathrm{O}$ & 2.66 & 3.05 & 6.67 & 7.55 & 10.74 & 12.26 & 0.45 & 0.46 \\
\hline S1006 & $\operatorname{c} 1 \mathrm{cn} 2 \mathrm{c}([\mathrm{nH}] 1) \operatorname{ccn} 2$ & -4.54 & -3.99 & 6.29 & 5.29 & 18.97 & 17.06 & 0.42 & 0.45 \\
\hline S10062 & $=\mathrm{c} 1[\mathrm{nH}] \mathrm{c} 2 \mathrm{cn}[\mathrm{nH}] \mathrm{c}(=\mathrm{O}) \mathrm{c} 2 \mathrm{c}(=\mathrm{O})[\mathrm{nH}] 1$ & 3.39 & 3.82 & 12.28 & 10.04 & 22.77 & 18.11 & 0.60 & 0.61 \\
\hline S10063 & $\mathrm{O}=\mathrm{c} 1[\mathrm{nH}] \mathrm{c}(=\mathrm{O}) \mathrm{c} 2 \mathrm{c}([\mathrm{nH}] 1) \mathrm{c}(=\mathrm{O})[\mathrm{nH}] \mathrm{nc} 2$ & 4.08 & 3.82 & 9.43 & 9.25 & 12.99 & 13.94 & 0.75 & 0.72 \\
\hline S1007 & {$[\mathrm{nH}] 1 \mathrm{cc} 2 \mathrm{c}(\mathrm{c} 1) \mathrm{cn}[\mathrm{nH}] 2$} & -5.51 & -4.74 & 4.58 & 5.55 & 16.35 & 17.60 & 0.48 & 0.47 \\
\hline S10077 & $\mathrm{O}=\mathrm{c} 1[\mathrm{nH}] \mathrm{ncc} 2 \mathrm{c} 1 \mathrm{nccn} 2$ & 1.27 & 2.28 & 6.36 & 6.71 & 15.74 & 12.15 & 0.47 & 0.45 \\
\hline S1008 & $\operatorname{c} 1 \mathrm{cc} 2 \mathrm{n}(\mathrm{n} 1) \operatorname{occ} 2$ & -2.82 & -2.39 & 4.31 & 4.13 & 13.70 & 13.13 & 0.47 & 0.45 \\
\hline S1009 & $\mathrm{c} 1 \mathrm{cn} 2 \mathrm{c}(\mathrm{o} 1) \mathrm{ccn} 2$ & -2.79 & -2.39 & 3.99 & 3.76 & 12.72 & 12.65 & 0.33 & 0.33 \\
\hline S1010 & $\mathrm{o} 1 \mathrm{cc} 2 \mathrm{c}(\mathrm{c} 1) \mathrm{cn}[\mathrm{nH}] 2$ & -3.39 & -3.14 & 2.24 & 3.95 & 9.23 & 13.30 & 0.51 & 0.51 \\
\hline S10113 & $\mathrm{O}=\mathrm{c} 1 \mathrm{cn}[\mathrm{nH}] \mathrm{c} 2 \mathrm{c} 1 \mathrm{cn}[\mathrm{nH}] \mathrm{c} 2=\mathrm{O}$ & 2.89 & 3.05 & 7.67 & 9.47 & 13.53 & 14.98 & 0.49 & 0.49 \\
\hline
\end{tabular}




\begin{tabular}{|c|c|c|c|c|c|c|c|c|c|}
\hline S1012 & $\mathrm{c} 1 \mathrm{cn} 2 \mathrm{c}(\mathrm{s} 1) \mathrm{ccn} 2$ & -3.37 & -3.08 & 3.03 & 3.05 & 11.44 & 12.16 & 0.41 & 0.35 \\
\hline S1013 & $\operatorname{s} 1 \mathrm{cc} 2 \mathrm{c}(\mathrm{c} 1) \mathrm{cn}[\mathrm{nH}] 2$ & -3.81 & -3.83 & 2.14 & 3.24 & 9.48 & 12.80 & 0.48 & 0.47 \\
\hline S1014 & $\mathrm{n} 1 \mathrm{cc} 2 \mathrm{c}([\mathrm{nH}] 1)[\mathrm{nH}] \mathrm{nc} 2$ & -2.99 & -2.91 & 6.02 & 7.00 & 13.03 & 16.91 & 0.48 & 0.54 \\
\hline S10148 & $\mathrm{O}=\mathrm{c} 1 \mathrm{nccc} 2 \mathrm{n} 1[\mathrm{nH}] \mathrm{cc} 2$ & -0.90 & -1.01 & 9.62 & 7.93 & 23.91 & 19.67 & 0.41 & 0.42 \\
\hline S1015 & $\mathrm{n} 1 \mathrm{cc} 2 \mathrm{c}([\mathrm{nH}] 1) \mathrm{cn}[\mathrm{nH}] 2$ & -3.13 & -2.91 & 2.68 & 4.87 & 9.27 & 14.59 & 0.60 & 0.62 \\
\hline S10156 & $\mathrm{O}=\mathrm{c} 1 \mathrm{ncc} 2 \mathrm{c}([\mathrm{nH}] 1) \csc 2$ & 0.21 & -0.85 & 6.14 & 7.30 & 18.88 & 18.56 & 0.69 & 0.70 \\
\hline S10157 & $\mathrm{O}=\mathrm{c} 1 \mathrm{nccc} 2 \mathrm{n} 1[\mathrm{nH}] \mathrm{nc} 2$ & 1.06 & 0.82 & 7.43 & 8.78 & 18.83 & 18.68 & 0.37 & 0.37 \\
\hline S1016 & $\mathrm{c} 1 \mathrm{nn} 2 \mathrm{c}(\mathrm{c} 1) \mathrm{cn}[\mathrm{nH}] 2$ & -2.61 & -2.16 & 3.53 & 5.38 & 10.22 & 14.58 & 0.46 & 0.38 \\
\hline S10160 & $\mathrm{O}=\mathrm{c} 1 \mathrm{ncc} 2 \mathrm{c}([\mathrm{nH}] 1) \mathrm{n}[\mathrm{nH}] \mathrm{c} 2$ & 0.07 & 0.07 & 10.97 & 10.01 & 25.03 & 21.93 & 0.48 & 0.51 \\
\hline S10169 & $\mathrm{O}=\mathrm{c} 1 \mathrm{nccc} 2 \mathrm{n} 1[\mathrm{nH}] \mathrm{cn} 2$ & 0.76 & 0.82 & 11.78 & 9.83 & 23.42 & 19.27 & 0.51 & 0.46 \\
\hline S1017 & $\mathrm{c} 1 \mathrm{nn} 2 \mathrm{c}(\mathrm{c} 1)[\mathrm{nH}] \mathrm{nc} 2$ & -2.45 & -2.16 & 5.31 & 6.43 & 13.72 & 16.72 & 0.35 & 0.33 \\
\hline S10175 & $\mathrm{O}=\mathrm{c} 1 \mathrm{nccc} 2 \mathrm{n} 1[\mathrm{nH}] \mathrm{nn} 2$ & 2.82 & 2.65 & 9.80 & 11.31 & 18.06 & 19.68 & 0.46 & 0.43 \\
\hline S10177 & $\mathrm{O}=\mathrm{c} 1 \mathrm{ncc} 2 \mathrm{c}([\mathrm{nH}] 1) \mathrm{n}[\mathrm{nH}] \mathrm{n} 2$ & 1.71 & 1.90 & 9.24 & 10.35 & 20.30 & 19.33 & 0.55 & 0.54 \\
\hline S1018 & $\mathrm{n} 1 \mathrm{cc} 2 \mathrm{c}([\mathrm{nH}] 1) \mathrm{n}[\mathrm{nH}] \mathrm{c} 2$ & -3.67 & -2.91 & 4.81 & 5.22 & 13.50 & 15.09 & 0.50 & 0.54 \\
\hline S1019 & $\mathrm{n} 1 \mathrm{cc} 2 \mathrm{c}([\mathrm{nH}] 1) \mathrm{c}[\mathrm{nH}] \mathrm{n} 2$ & -3.72 & -2.91 & 5.00 & 7.13 & 13.92 & 18.04 & 0.53 & 0.52 \\
\hline $\mathbf{S 1 0 2 0}$ & $\mathrm{n} 1 \mathrm{oc} 2 \mathrm{c}(\mathrm{c} 1) \mathrm{cn}[\mathrm{nH}] 2$ & -1.00 & -1.31 & 4.62 & 5.09 & 10.99 & 12.97 & 0.33 & 0.32 \\
\hline $\mathbf{S 1 0 2 0 2}$ & $\mathrm{O}=\mathrm{c} 1[\mathrm{nH}] \mathrm{cc} 2 \mathrm{c}(\mathrm{c} 1) \mathrm{cnc}(=\mathrm{O})[\mathrm{nH}] 2$ & 3.10 & 1.22 & 11.67 & 12.04 & 20.97 & 22.93 & 0.44 & 0.48 \\
\hline S10203 & $\mathrm{O}=\mathrm{c} 1[\mathrm{nH}] \mathrm{cc} 2 \mathrm{c}(\mathrm{c} 1)[\mathrm{nH}] \mathrm{c}(=\mathrm{O}) \mathrm{nc} 2$ & 2.88 & 1.22 & 12.99 & 10.32 & 23.01 & 20.73 & 0.36 & 0.32 \\
\hline S1021 & $\mathrm{n} 1 \mathrm{cc} 2 \mathrm{c}(\mathrm{o} 1) \mathrm{cn}[\mathrm{nH}] 2$ & -1.06 & -1.31 & 6.91 & 5.84 & 13.91 & 13.49 & 0.50 & 0.48 \\
\hline S1023 & c1nn2c(c1)onc2 & -0.45 & -0.56 & 3.86 & 5.86 & 7.79 & 13.65 & 0.44 & 0.44 \\
\hline S1024 & $\mathrm{n} 1 \mathrm{cc} 2 \mathrm{c}([\mathrm{nH}] 1)$ noc2 & -1.22 & -1.31 & 4.05 & 4.58 & 11.09 & 12.13 & 0.47 & 0.58 \\
\hline S1025 & $\mathrm{n} 1 \mathrm{cc} 2 \mathrm{c}([\mathrm{nH}] 1) \operatorname{con} 2$ & -1.29 & -1.31 & 6.54 & 6.49 & 14.60 & 15.08 & 0.37 & 0.38 \\
\hline S10254 & $\mathrm{O}=\mathrm{c} 1 \mathrm{ncc} 2 \mathrm{c}([\mathrm{nH}] 1) \mathrm{ncc}(=\mathrm{O})[\mathrm{nH}] 2$ & 4.79 & 3.05 & 11.87 & 10.52 & 21.59 & 18.89 & 0.57 & 0.55 \\
\hline S10255 & $\mathrm{O}=\mathrm{c} 1 \mathrm{ncc} 2 \mathrm{c}([\mathrm{nH}] 1)[\mathrm{nH}] \mathrm{c}(=\mathrm{O}) \mathrm{cn} 2$ & 4.76 & 3.05 & 14.57 & 14.04 & 22.98 & 22.25 & 0.46 & 0.46 \\
\hline S1026 & $\mathrm{n} 1 \mathrm{sc} 2 \mathrm{c}(\mathrm{c} 1) \mathrm{cn}[\mathrm{nH}] 2$ & -1.75 & -2.00 & 5.20 & 4.40 & 11.40 & 12.42 & 0.51 & 0.49 \\
\hline $\mathrm{S} 10262$ & $\mathrm{O}=\mathrm{c} 1 \mathrm{ncc} 2 \mathrm{c}([\mathrm{nH}] 1) \mathrm{c}(=\mathrm{O})[\mathrm{nH}] \mathrm{c}(=\mathrm{O})[\mathrm{nH}] 2$ & 5.50 & 3.82 & 13.12 & 11.82 & 22.43 & 20.12 & 0.46 & 0.45 \\
\hline S10263 & $\mathrm{O}=\mathrm{c} 1 \mathrm{ncc} 2 \mathrm{c}([\mathrm{nH}] 1)[\mathrm{nH}] \mathrm{c}(=\mathrm{O})[\mathrm{nH}] \mathrm{c} 2=\mathrm{O}$ & 5.42 & 3.82 & 15.82 & 14.68 & 23.68 & 23.77 & 0.43 & 0.46 \\
\hline S1027 & $\mathrm{n} 1 \mathrm{cc} 2 \mathrm{c}(\mathrm{s} 1) \mathrm{cn}[\mathrm{nH}] 2$ & -1.73 & -2.00 & 5.97 & 4.96 & 12.08 & 12.59 & 0.48 & 0.52 \\
\hline S1029 & $\mathrm{c} 1 \mathrm{nn} 2 \mathrm{c}(\mathrm{c} 1) \mathrm{snc} 2$ & -1.30 & -1.25 & 1.79 & 5.08 & 6.50 & 12.99 & 0.41 & 0.39 \\
\hline S10292 & $\mathrm{O}=\mathrm{c} 1 \mathrm{ncc} 2 \mathrm{c}([\mathrm{nH}] 1) \operatorname{nccn} 2$ & 2.63 & 2.28 & 7.94 & 9.53 & 18.34 & 18.26 & 0.40 & 0.41 \\
\hline S10294 & $\mathrm{O}=\mathrm{c} 1 \mathrm{ncc} 2 \mathrm{c}([\mathrm{nH}] 1) \mathrm{ncnn} 2$ & 4.72 & 4.11 & 11.00 & 11.04 & 17.51 & 18.06 & 0.60 & 0.63 \\
\hline
\end{tabular}




\begin{tabular}{|c|c|c|c|c|c|c|c|c|c|}
\hline $\mathbf{S 1 0 2 9 5}$ & $\mathrm{O}=\mathrm{c} 1 \mathrm{ncc} 2 \mathrm{c}([\mathrm{nH}] 1) \mathrm{nncn} 2$ & 4.69 & 4.11 & 10.97 & 10.26 & 18.02 & 17.05 & 0.51 & 0.50 \\
\hline $\mathbf{S 1 0 3 0}$ & $\mathrm{n} 1 \mathrm{cc} 2 \mathrm{c}([\mathrm{nH}] 1) \mathrm{nsc} 2$ & -2.01 & -2.00 & 3.45 & 3.81 & 8.86 & 11.46 & 0.70 & 0.68 \\
\hline S1032 & $\mathrm{c} 1 \mathrm{nc} 2 \mathrm{c}([\mathrm{nH}] 1)[\mathrm{nH}] \mathrm{nc} 2$ & -3.18 & -2.91 & 9.31 & 9.92 & 19.72 & 21.71 & 0.39 & 0.39 \\
\hline S1033 & $\mathrm{c} 1[\mathrm{nH}] \mathrm{c} 2 \mathrm{c}(\mathrm{n} 1)[\mathrm{nH}] \mathrm{nc} 2$ & -3.30 & -2.91 & 6.78 & 5.88 & 15.68 & 16.43 & 0.79 & 0.81 \\
\hline S1034 & $\mathrm{c} 1 \mathrm{nn} 2 \mathrm{c}(\mathrm{c} 1) \mathrm{nc}[\mathrm{nH}] 2$ & -2.82 & -2.16 & 8.48 & 6.65 & 17.03 & 16.18 & 0.51 & 0.54 \\
\hline S1035 & $\operatorname{c} 1 \mathrm{cc} 2 \mathrm{n}(\mathrm{n} 1) \mathrm{nc}[\mathrm{nH}] 2$ & -2.71 & -2.16 & 9.99 & 8.64 & 23.01 & 21.04 & 0.71 & 0.68 \\
\hline S1036 & $\mathrm{c} 1 \mathrm{nc} 2 \mathrm{c}(\mathrm{o} 1)[\mathrm{nH}] \mathrm{nc} 2$ & -1.45 & -1.31 & 5.18 & 5.51 & 11.37 & 14.27 & 0.39 & 0.37 \\
\hline S1037 & $\mathrm{c} 1 \mathrm{oc} 2 \mathrm{c}(\mathrm{n} 1)[\mathrm{nH}] \mathrm{nc} 2$ & -1.50 & -1.31 & 2.84 & 4.35 & 8.82 & 11.83 & 0.59 & 0.56 \\
\hline S1039 & $\operatorname{c1cc} 2 n(n 1) n c o 2$ & -0.99 & -0.56 & 6.06 & 5.58 & 14.98 & 14.46 & 0.39 & 0.39 \\
\hline S104 & $\mathrm{O}=\mathrm{c} 1 \mathrm{ccscn} 1$ & 0.64 & 0.49 & 7.14 & 5.99 & 18.42 & 16.93 & 0.55 & 0.55 \\
\hline S1040 & $\mathrm{c} 1 \mathrm{nc} 2 \mathrm{c}(\mathrm{s} 1)[\mathrm{nH}] \mathrm{nc} 2$ & -2.20 & -2.00 & 4.92 & 4.98 & 11.37 & 14.15 & 0.59 & 0.62 \\
\hline S1041 & $\mathrm{c} 1 \mathrm{sc} 2 \mathrm{c}(\mathrm{n} 1)[\mathrm{nH}] \mathrm{nc} 2$ & -2.19 & -2.00 & 2.18 & 3.64 & 8.38 & 11.36 & 0.81 & 0.74 \\
\hline S1043 & $\operatorname{c1cc} 2 n(n 1) n c s 2$ & -1.80 & -1.25 & 4.83 & 4.96 & 14.36 & 14.24 & 0.35 & 0.36 \\
\hline S1044 & $\mathrm{n} 1[\mathrm{nH}] \mathrm{c} 2 \mathrm{c}(\mathrm{c} 1) \mathrm{nn}[\mathrm{nH}] 2$ & -1.11 & -1.08 & 9.32 & 11.07 & 18.86 & 21.61 & 0.31 & 0.30 \\
\hline S1045 & $\mathrm{n} 1[\mathrm{nH}] \mathrm{c} 2 \mathrm{c}(\mathrm{c} 1)[\mathrm{nH}] \mathrm{nn} 2$ & -1.22 & -1.08 & 6.32 & 7.03 & 14.32 & 16.33 & 0.36 & 0.35 \\
\hline S1046 & $\mathrm{c} 1 \mathrm{nn} 2 \mathrm{c}(\mathrm{c} 1) \mathrm{nn}[\mathrm{nH}] 2$ & -0.76 & -0.33 & 7.33 & 8.42 & 13.23 & 17.23 & 0.42 & 0.41 \\
\hline S1047 & $\mathrm{c} 1 \mathrm{nn} 2 \mathrm{c}(\mathrm{c} 1)[\mathrm{nH}] \mathrm{nn} 2$ & -0.69 & -0.33 & 9.26 & 10.42 & 18.29 & 22.08 & 0.42 & 0.43 \\
\hline S1048 & {$[\mathrm{nH}] 1 \mathrm{nc} 2 \mathrm{c}(\mathrm{n} 1) \mathrm{cn}[\mathrm{nH}] 2$} & -1.89 & -1.08 & 3.83 & 5.79 & 9.25 & 13.28 & 0.49 & 0.49 \\
\hline S1050 & $\mathrm{n} 1 \mathrm{oc} 2 \mathrm{c}(\mathrm{n} 1)[\mathrm{nH}] \mathrm{nc} 2$ & 0.69 & 0.52 & 6.45 & 6.46 & 11.66 & 13.07 & 0.53 & 0.51 \\
\hline S1052 & c1nn2c(c1)onn2 & 1.20 & 1.27 & 4.31 & 8.30 & 8.51 & 16.85 & 0.49 & 0.50 \\
\hline S1053 & $\mathrm{n} 1 \mathrm{nc} 2 \mathrm{c}(\mathrm{s} 1)[\mathrm{nH}] \mathrm{nc} 2$ & -0.14 & -0.17 & 7.84 & 7.03 & 14.49 & 15.22 & 0.45 & 0.47 \\
\hline S1054 & $\mathrm{n} 1 \mathrm{sc} 2 \mathrm{c}(\mathrm{n} 1)[\mathrm{nH}] \mathrm{nc} 2$ & -0.08 & -0.17 & 4.54 & 5.68 & 10.34 & 12.43 & 0.40 & 0.41 \\
\hline S10558 & $\mathrm{O}=\mathrm{c} 1 \mathrm{ncc} 2 \mathrm{c}(\mathrm{o} 1) \csc 2$ & 1.24 & 0.75 & 8.13 & 7.10 & 19.61 & 17.05 & 0.60 & 0.57 \\
\hline S1056 & $\mathrm{c} 1 \mathrm{nn} 2 \mathrm{c}(\mathrm{c} 1) \operatorname{snn} 2$ & 0.21 & 0.58 & 4.63 & 7.63 & 10.80 & 16.46 & 0.47 & 0.50 \\
\hline S1057 & $\mathrm{O}=\mathrm{c} 1 \mathrm{c}(=\mathrm{O}) \mathrm{c} 2 \mathrm{c}(\mathrm{c} 1=\mathrm{O})[\mathrm{nH}] \mathrm{nc} 2$ & 4.79 & 5.54 & 11.19 & 11.73 & 16.63 & 20.94 & 0.82 & 0.80 \\
\hline S1059 & $\mathrm{o} 1 \mathrm{nc} 2 \mathrm{c}(\mathrm{n} 1) \mathrm{cn}[\mathrm{nH}] 2$ & 0.87 & 0.52 & 6.48 & 6.10 & 11.63 & 11.66 & 0.41 & 0.41 \\
\hline S1060 & $\operatorname{s} 1 \mathrm{nc} 2 \mathrm{c}(\mathrm{n} 1) \mathrm{cn}[\mathrm{nH}] 2$ & -0.24 & -0.17 & 4.24 & 5.27 & 7.33 & 10.82 & 0.45 & 0.47 \\
\hline S1061 & $\mathrm{O}=\mathrm{c} 1[\mathrm{nH}] \mathrm{c} 2 \mathrm{c}([\mathrm{nH}] 1) \mathrm{cn}[\mathrm{nH}] 2$ & -1.32 & -2.14 & 6.88 & 7.23 & 15.75 & 17.86 & 0.55 & 0.52 \\
\hline S1067 & $\mathrm{O}=\mathrm{c} 1[\mathrm{nH}] \mathrm{c} 2 \mathrm{c}(\mathrm{s} 1)[\mathrm{nH}] \mathrm{nc} 2$ & -0.69 & -1.23 & 5.19 & 6.34 & 12.71 & 15.34 & 0.35 & 0.37 \\
\hline S1068 & $\mathrm{O}=\mathrm{c} 1 \mathrm{sc} 2 \mathrm{c}([\mathrm{nH}] 1)[\mathrm{nH}] \mathrm{nc} 2$ & -0.53 & -1.23 & 9.40 & 9.03 & 16.86 & 17.83 & 0.58 & 0.58 \\
\hline S1071 & $\mathrm{c} 1 \mathrm{ccc} 2 \mathrm{c}(\mathrm{c} 1)[\mathrm{nH}] \mathrm{nc} 2$ & -4.06 & -4.36 & 2.49 & 3.37 & 10.23 & 13.78 & 0.60 & 0.59 \\
\hline
\end{tabular}




\begin{tabular}{|c|c|c|c|c|c|c|c|c|c|}
\hline S1072 & $\mathrm{c} 1 \mathrm{ccc} 2 \mathrm{n}(\mathrm{c} 1) \mathrm{ncc} 2$ & -3.67 & -3.61 & 1.79 & 3.17 & 10.61 & 13.15 & 0.63 & 0.67 \\
\hline S1073 & $\mathrm{c} 1 \mathrm{ncc} 2 \mathrm{c}(\mathrm{c} 1)[\mathrm{nH}] \mathrm{nc} 2$ & -2.09 & -2.53 & 5.30 & 5.03 & 12.69 & 14.13 & 0.56 & 0.56 \\
\hline S1074 & $\operatorname{c1ncc} 2 \mathrm{c}(\mathrm{c} 1) \mathrm{cn}[\mathrm{nH}] 2$ & -2.13 & -2.53 & 4.70 & 4.28 & 11.47 & 12.97 & 0.43 & 0.43 \\
\hline S1075 & $\mathrm{c} 1 \mathrm{cnc} 2 \mathrm{c}(\mathrm{c} 1) \mathrm{cn}[\mathrm{nH}] 2$ & -2.54 & -2.53 & 2.26 & 3.79 & 8.37 & 12.56 & 0.48 & 0.52 \\
\hline S1076 & $\mathrm{c} 1 \mathrm{cnc} 2 \mathrm{c}(\mathrm{c} 1)[\mathrm{nH}] \mathrm{nc} 2$ & -2.51 & -2.53 & 5.23 & 5.84 & 13.27 & 15.74 & 0.51 & 0.51 \\
\hline S1077 & $\mathrm{n} 1 \mathrm{ccn} 2 \mathrm{c}(\mathrm{c} 1) \operatorname{ccn} 2$ & -1.74 & -1.78 & 1.53 & 4.75 & 6.63 & 13.31 & 0.42 & 0.42 \\
\hline S1078 & $\mathrm{c} 1 \mathrm{ncn} 2 \mathrm{c}(\mathrm{c} 1) \mathrm{ccn} 2$ & -1.71 & -1.78 & 2.88 & 5.22 & 8.47 & 14.51 & 0.40 & 0.39 \\
\hline S1079 & $\mathrm{c} 1 \mathrm{cnn} 2 \mathrm{c}(\mathrm{c} 1) \mathrm{ccn} 2$ & -2.16 & -1.78 & 4.65 & 5.39 & 15.42 & 15.53 & 0.47 & 0.45 \\
\hline $\mathbf{S 1 0 8 0}$ & $\mathrm{c} 1 \mathrm{ccn} 2 \mathrm{c}(\mathrm{n} 1) \operatorname{ccn} 2$ & -2.18 & -1.78 & 4.21 & 4.30 & 10.43 & 12.50 & 0.56 & 0.57 \\
\hline S1081 & $\mathrm{c} 1 \mathrm{nnc} 2 \mathrm{c}(\mathrm{c} 1)[\mathrm{nH}] \mathrm{nc} 2$ & -0.45 & -0.70 & 8.46 & 8.52 & 18.23 & 18.34 & 0.56 & 0.57 \\
\hline S1082 & $\mathrm{c} 1 \mathrm{nnn} 2 \mathrm{c}(\mathrm{c} 1) \mathrm{cn}[\mathrm{nH}] 2$ & -0.52 & -0.70 & 4.86 & 5.72 & 13.05 & 14.01 & 0.42 & 0.40 \\
\hline S1083 & $\mathrm{n} 1 \mathrm{ccn} 2 \mathrm{c}(\mathrm{n} 1) \operatorname{ccn} 2$ & -0.20 & 0.05 & 5.35 & 6.91 & 12.19 & 14.91 & 0.34 & 0.33 \\
\hline S1085 & $\mathrm{n} 1 \mathrm{ncc} 2 \mathrm{c}(\mathrm{c} 1)[\mathrm{nH}] \mathrm{nc} 2$ & 0.04 & -0.70 & 8.01 & 6.96 & 18.01 & 15.57 & 0.47 & 0.38 \\
\hline S1086 & $\mathrm{n} 1 \mathrm{ncc} 2 \mathrm{n}(\mathrm{c} 1) \mathrm{ncc} 2$ & 0.47 & 0.05 & 4.90 & 7.82 & 12.40 & 16.92 & 0.74 & 0.70 \\
\hline S1087 & $\mathrm{O}=\mathrm{c} 1[\mathrm{nH}] \operatorname{ccc} 2 \mathrm{c} 1 \mathrm{cn}[\mathrm{nH}] 2$ & -1.84 & -1.76 & 6.62 & 7.78 & 17.73 & 19.45 & 0.51 & 0.51 \\
\hline S1088 & $\mathrm{O}=\mathrm{c} 1[\mathrm{nH}] \mathrm{ccc} 2 \mathrm{c} 1[\mathrm{nH}] \mathrm{nc} 2$ & -1.67 & -1.76 & 5.53 & 6.36 & 13.30 & 15.91 & 0.52 & 0.54 \\
\hline S1089 & $\mathrm{O}=\mathrm{c} 1 \mathrm{ccc} 2 \mathrm{c}([\mathrm{nH}] 1)[\mathrm{nH}] \mathrm{nc} 2$ & -0.67 & -1.76 & 8.08 & 7.45 & 17.45 & 16.81 & 0.72 & 0.61 \\
\hline $\mathbf{S 1 0 9 0}$ & $\mathrm{O}=\mathrm{c} 1 \mathrm{ccc} 2 \mathrm{c}([\mathrm{nH}] 1) \mathrm{cn}[\mathrm{nH}] 2$ & -0.76 & -1.76 & 7.25 & 5.98 & 17.87 & 15.93 & 0.61 & 0.57 \\
\hline S1091 & $\mathrm{O}=\mathrm{c} 1[\mathrm{nH}] \operatorname{ccn} 2 \mathrm{c} 1 \mathrm{ccn} 2$ & -1.59 & -1.01 & 7.06 & 6.19 & 15.58 & 15.31 & 0.38 & 0.37 \\
\hline S1092 & $\mathrm{O}=\mathrm{c} 1[\mathrm{nH}] \operatorname{ccc} 2 \mathrm{n} 1 \mathrm{ncc} 2$ & -1.72 & -1.01 & 7.70 & 6.86 & 20.22 & 18.61 & 0.48 & 0.43 \\
\hline S1094 & $\mathrm{O}=\mathrm{c} 1 \mathrm{ccn} 2 \mathrm{c}([\mathrm{nH}] 1) \operatorname{ccn} 2$ & -0.34 & -1.01 & 4.29 & 7.18 & 12.15 & 17.09 & 0.57 & 0.56 \\
\hline S1097 & $\mathrm{O}=\mathrm{c} 1 \mathrm{occc} 2 \mathrm{c} 1 \mathrm{cn}[\mathrm{nH}] 2$ & -0.20 & -0.16 & 8.51 & 8.53 & 18.76 & 18.90 & 0.65 & 0.67 \\
\hline S1098 & $\mathrm{O}=\mathrm{c} 1 \mathrm{occc} 2 \mathrm{c} 1[\mathrm{nH}] \mathrm{nc} 2$ & -0.06 & -0.16 & 4.49 & 5.98 & 12.02 & 14.25 & 0.57 & 0.55 \\
\hline S10999 & $\mathrm{O}=\mathrm{c} 1 \mathrm{cnc} 2 \mathrm{c}([\mathrm{nH}] 1) \operatorname{coc} 2$ & 0.16 & -0.16 & 5.33 & 6.86 & 12.58 & 14.84 & 0.79 & 0.83 \\
\hline S11 & $\mathrm{c} 1 \mathrm{cn}[\mathrm{nH}] \mathrm{n} 1$ & -2.10 & -1.57 & 2.67 & 3.26 & 7.05 & 11.08 & 0.50 & 0.50 \\
\hline S11002 & $\mathrm{O}=\mathrm{c} 1 \mathrm{cnc} 2 \mathrm{c}([\mathrm{nH}] 1) \csc 2$ & -0.32 & -0.85 & 5.29 & 6.17 & 12.94 & 14.41 & 0.45 & 0.44 \\
\hline S11005 & $\mathrm{O}=\mathrm{c} 1 \mathrm{cnc} 2 \mathrm{c}([\mathrm{nH}] 1) \mathrm{n}[\mathrm{nH}] \mathrm{c} 2$ & -0.39 & 0.07 & 8.23 & 7.87 & 17.28 & 16.24 & 0.54 & 0.51 \\
\hline S11029 & $\mathrm{O}=\mathrm{c} 1 \mathrm{cnc} 2 \mathrm{c}([\mathrm{nH}] 1)$ non 2 & 4.44 & 3.50 & 10.55 & 9.15 & 13.46 & 13.45 & 0.65 & 0.61 \\
\hline S11030 & $\mathrm{O}=\mathrm{c} 1 \mathrm{cnc} 2 \mathrm{c}([\mathrm{nH}] 1) \mathrm{nsn} 2$ & 3.09 & 2.81 & 7.73 & 8.33 & 11.49 & 12.68 & 0.47 & 0.46 \\
\hline S11036 & $\mathrm{O}=\mathrm{c} 1 \mathrm{cncc} 2 \mathrm{n} 1 \mathrm{ccc} 2$ & -0.27 & -0.63 & 6.73 & 5.81 & 15.98 & 14.55 & 0.80 & 0.83 \\
\hline S11038 & $\mathrm{O}=\mathrm{c} 1 \mathrm{cncc} 2 \mathrm{n} 1 \mathrm{cncc} 2$ & 1.63 & 1.20 & 6.82 & 7.55 & 13.50 & 15.50 & 0.66 & 0.66 \\
\hline
\end{tabular}




\begin{tabular}{|c|c|c|c|c|c|c|c|c|c|}
\hline S11048 & $\mathrm{O}=\mathrm{c} 1[\mathrm{nH}] \mathrm{cc} 2 \mathrm{c}(\mathrm{c} 1)[\mathrm{nH}] \mathrm{c}(=\mathrm{O}) \mathrm{cn} 2$ & 2.14 & 1.22 & 8.34 & 8.52 & 15.94 & 16.04 & 0.36 & 0.36 \\
\hline S11049 & $\mathrm{O}=\mathrm{c} 1[\mathrm{nH}] \mathrm{cc} 2 \mathrm{c}(\mathrm{c} 1) \mathrm{ncc}(=\mathrm{O})[\mathrm{nH}] 2$ & 2.36 & 1.22 & 11.36 & 11.24 & 20.38 & 20.40 & 0.61 & 0.57 \\
\hline S11072 & $\mathrm{O}=\mathrm{c} 1[\mathrm{nH}] \mathrm{cc} 2 \mathrm{c}(\mathrm{n} 1) \mathrm{ncc}(=\mathrm{O})[\mathrm{nH}] 2$ & 3.85 & 3.05 & 14.91 & 16.28 & 26.21 & 28.03 & 0.51 & 0.48 \\
\hline S11073 & $\mathrm{O}=\mathrm{c} 1[\mathrm{nH}] \mathrm{cc} 2 \mathrm{c}(\mathrm{n} 1)[\mathrm{nH}] \mathrm{c}(=\mathrm{O}) \mathrm{cn} 2$ & 3.29 & 3.05 & 11.31 & 10.30 & 20.25 & 18.37 & 0.73 & 0.67 \\
\hline S1109 & $\mathrm{O}=\mathrm{c} 1 \mathrm{ccc} 2 \mathrm{c}(\mathrm{s} 1)[\mathrm{nH}] \mathrm{nc} 2$ & -0.38 & -0.85 & 5.35 & 5.59 & 13.23 & 13.97 & 0.34 & 0.36 \\
\hline S11091 & $\mathrm{O}=\mathrm{c} 1 \mathrm{cnc} 2 \mathrm{c}([\mathrm{nH}] 1) \mathrm{ncc}(=\mathrm{O})[\mathrm{nH}] 2$ & 4.02 & 3.05 & 9.06 & 8.69 & 14.17 & 14.49 & 0.44 & 0.45 \\
\hline S11098 & $\mathrm{O}=\mathrm{c} 1 \mathrm{cnc} 2 \mathrm{c}([\mathrm{nH}] 1)[\mathrm{nH}] \mathrm{c}(=\mathrm{O})[\mathrm{nH}] \mathrm{c} 2=\mathrm{O}$ & 4.68 & 3.82 & 15.44 & 14.20 & 23.21 & 22.17 & 0.33 & 0.31 \\
\hline S11099 & $\mathrm{O}=\mathrm{c} 1 \mathrm{cnc} 2 \mathrm{c}([\mathrm{nH}] 1) \mathrm{c}(=\mathrm{O})[\mathrm{nH}] \mathrm{c}(=\mathrm{O})[\mathrm{nH}] 2$ & 4.74 & 3.82 & 10.32 & 9.70 & 15.35 & 14.96 & 0.33 & 0.34 \\
\hline $\mathbf{S 1 1 1 0 2}$ & $\mathrm{O}=\mathrm{c} 1 \mathrm{cnc} 2 \mathrm{c}([\mathrm{nH}] 1)[\mathrm{nH}] \mathrm{c}(=\mathrm{O}) \mathrm{c}(=\mathrm{O})[\mathrm{nH}] 2$ & 5.18 & 3.82 & 14.75 & 13.75 & 22.60 & 24.58 & 0.45 & 0.46 \\
\hline S11112 & $\mathrm{O}=\mathrm{c} 1 \mathrm{cnc} 2 \mathrm{c}([\mathrm{nH}] 1)[\mathrm{nH}] \mathrm{c}(=\mathrm{O}) \mathrm{sc} 2=\mathrm{O}$ & 5.16 & 4.73 & 16.39 & 16.75 & 23.06 & 24.17 & 0.50 & 0.49 \\
\hline S11113 & $\mathrm{O}=\mathrm{c} 1 \mathrm{cnc} 2 \mathrm{c}([\mathrm{nH}] 1) \mathrm{c}(=\mathrm{O}) \mathrm{sc}(=\mathrm{O})[\mathrm{nH}] 2$ & 5.18 & 4.73 & 11.39 & 10.51 & 15.46 & 15.27 & 0.38 & 0.38 \\
\hline $\mathbf{S 1 1 1 2 0}$ & $\mathrm{O}=\mathrm{c} 1 \mathrm{cnc} 2 \mathrm{c}([\mathrm{nH}] 1) \mathrm{c}(=\mathrm{O}) \mathrm{cc}[\mathrm{nH}] 2$ & 1.42 & 1.22 & 10.89 & 8.78 & 19.63 & 18.29 & 0.47 & 0.51 \\
\hline S11121 & $\mathrm{O}=\mathrm{c} 1 \mathrm{cnc} 2 \mathrm{c}([\mathrm{nH}] 1)[\mathrm{nH}] \mathrm{ccc} 2=\mathrm{O}$ & 1.54 & 1.22 & 16.23 & 16.00 & 30.08 & 29.87 & 0.46 & 0.46 \\
\hline S11124 & $\mathrm{O}=\mathrm{c} 1 \mathrm{cnc} 2 \mathrm{c}([\mathrm{nH}] 1) \mathrm{c}(=\mathrm{O}) \mathrm{nc}[\mathrm{nH}] 2$ & 3.32 & 3.05 & 12.72 & 11.09 & 21.96 & 20.80 & 0.63 & 0.62 \\
\hline S11125 & $\mathrm{O}=\mathrm{c} 1 \mathrm{cnc} 2 \mathrm{c}([\mathrm{nH}] 1)[\mathrm{nH}] \mathrm{cnc} 2=\mathrm{O}$ & 3.44 & 3.05 & 18.69 & 19.54 & 32.53 & 34.38 & 0.59 & 0.59 \\
\hline S11128 & $\mathrm{O}=\mathrm{c} 1 \mathrm{cnc} 2 \mathrm{c}([\mathrm{nH}] 1) \mathrm{nccn} 2$ & 2.14 & 2.28 & 5.93 & 8.58 & 11.55 & 14.79 & 0.41 & 0.40 \\
\hline $\mathbf{S 1 1 1 3 0}$ & $\mathrm{O}=\mathrm{c} 1 \mathrm{cnc} 2 \mathrm{c}([\mathrm{nH}] 1) \mathrm{nncn} 2$ & 4.30 & 4.11 & 10.15 & 9.55 & 12.56 & 14.09 & 0.46 & 0.46 \\
\hline S11131 & $\mathrm{O}=\mathrm{c} 1 \mathrm{cnc} 2 \mathrm{c}([\mathrm{nH}] 1) \mathrm{ncnn} 2$ & 4.37 & 4.11 & 10.60 & 10.77 & 15.64 & 16.08 & 0.59 & 0.57 \\
\hline S11134 & $\mathrm{O}=\mathrm{c} 1 \mathrm{cnc} 2 \mathrm{c}([\mathrm{nH}] 1) \mathrm{c}(=\mathrm{O})[\mathrm{nH}] \mathrm{cn} 2$ & 2.81 & 3.05 & 11.42 & 12.09 & 18.85 & 18.69 & 0.46 & 0.44 \\
\hline S11135 & $\mathrm{O}=\mathrm{c} 1 \mathrm{cnc} 2 \mathrm{c}([\mathrm{nH}] 1) \mathrm{nc}[\mathrm{nH}] \mathrm{c} 2=\mathrm{O}$ & 2.36 & 3.05 & 12.03 & 11.04 & 20.33 & 19.24 & 0.44 & 0.44 \\
\hline S11151 & $\mathrm{O}=\mathrm{c} 1 \mathrm{cnc} 2 \mathrm{c}([\mathrm{nH}] 1) \mathrm{ncsc} 2=\mathrm{O}$ & 3.17 & 3.96 & 9.17 & 10.24 & 16.04 & 16.62 & 0.62 & 0.63 \\
\hline S11154 & $\mathrm{O}=\mathrm{c} 1 \mathrm{cnc} 2 \mathrm{c}([\mathrm{nH}] 1) \mathrm{nnnn} 2$ & 6.65 & 5.94 & 14.81 & 12.76 & 19.62 & 17.62 & 0.62 & 0.60 \\
\hline S1117 & $\mathrm{c} 1 \mathrm{ncc} 2 \mathrm{c}(\mathrm{n} 1) \mathrm{cn}[\mathrm{nH}] 2$ & -0.56 & -0.70 & 7.53 & 6.16 & 13.00 & 13.64 & 0.50 & 0.51 \\
\hline S1118 & $\mathrm{c} 1 \mathrm{ncc} 2 \mathrm{c}(\mathrm{n} 1)[\mathrm{nH}] \mathrm{nc} 2$ & -0.54 & -0.70 & 2.97 & 4.85 & 7.27 & 11.62 & 0.55 & 0.58 \\
\hline S1119 & $\mathrm{c} 1 \mathrm{ncn} 2 \mathrm{c}(\mathrm{n} 1) \operatorname{ccn} 2$ & -0.18 & 0.05 & 2.85 & 5.76 & 5.97 & 12.57 & 0.52 & 0.47 \\
\hline $\mathbf{S 1 1 2 0}$ & $\mathrm{n} 1 \mathrm{cnn} 2 \mathrm{c}(\mathrm{c} 1) \mathrm{ccn} 2$ & -0.20 & 0.05 & 5.01 & 6.38 & 12.33 & 14.39 & 0.72 & 0.69 \\
\hline S1121 & $\mathrm{n} 1 \mathrm{nnc} 2 \mathrm{c}(\mathrm{c} 1)[\mathrm{nH}] \mathrm{nc} 2$ & 1.63 & 1.13 & 11.09 & 9.86 & 20.78 & 18.49 & 0.45 & 0.48 \\
\hline S1122 & $\mathrm{n} 1 \mathrm{nnc} 2 \mathrm{c}(\mathrm{c} 1) \mathrm{cn}[\mathrm{nH}] 2$ & 1.61 & 1.13 & 6.36 & 7.80 & 15.06 & 15.31 & 0.38 & 0.41 \\
\hline $\mathbf{S 1 1 2 3}$ & $\mathrm{n} 1 \mathrm{ncn} 2 \mathrm{c}(\mathrm{n} 1) \operatorname{ccn} 2$ & 1.98 & 1.88 & 6.02 & 9.39 & 12.70 & 17.23 & 0.49 & 0.49 \\
\hline S1125 & $\mathrm{O}=\mathrm{c} 1[\mathrm{nH}] \mathrm{ncc} 2 \mathrm{c} 1 \mathrm{cn}[\mathrm{nH}] 2$ & 0.00 & 0.07 & 8.85 & 6.89 & 18.12 & 15.05 & 0.38 & 0.39 \\
\hline S1126 & $\mathrm{O}=\mathrm{c} 1[\mathrm{nH}] \mathrm{ncc} 2 \mathrm{c} 1[\mathrm{nH}] \mathrm{nc} 2$ & 0.22 & 0.07 & 3.78 & 6.23 & 10.03 & 12.68 & 0.36 & 0.36 \\
\hline
\end{tabular}




\begin{tabular}{|c|c|c|c|c|c|c|c|c|}
\hline S11269 & $\mathrm{O}=\mathrm{c} 1 \mathrm{nnc} 2 \mathrm{c}([\mathrm{nH}] 1) \operatorname{coc} 2$ & 1.93 & 1.67 & 8.28 & 11.07 & 18.29 & 21.71 & 0.47 \\
\hline S1127 & $\mathrm{O}=\mathrm{c} 1[\mathrm{nH}] \mathrm{ncn} 2 \mathrm{c} 1 \mathrm{ccn} 2$ & 0.35 & 0.82 & 3.62 & 6.45 & 10.39 & 13.09 & 0.42 \\
\hline S11271 & $\mathrm{O}=\mathrm{c} 1[\mathrm{n}] \mathrm{ncc} 2 \mathrm{n} 1 \mathrm{ccs} 2$ & 2.18 & 1.73 & 10.75 & 9.61 & 22.90 & 19.90 & 0.46 \\
\hline S11272 & $\mathrm{O}=\mathrm{c} 1 \mathrm{nnc} 2 \mathrm{c}([\mathrm{nH}] 1) \mathrm{csc} 2$ & 1.47 & 0.98 & 8.15 & 10.43 & 18.72 & 21.41 & 0.48 \\
\hline S11275 & $\mathrm{O}=\mathrm{c} 1 \mathrm{nnc} 2 \mathrm{c}([\mathrm{nH}] 1) \mathrm{n}[\mathrm{nH}] \mathrm{c} 2$ & 1.45 & 1.90 & 11.83 & 12.39 & 23.43 & 23.12 & 0.69 \\
\hline S1128 & $\mathrm{O}=\mathrm{c} 1[\mathrm{nH}] \mathrm{ncc} 2 \mathrm{n} 1 \mathrm{ncc} 2$ & .16 & 0.82 & 5.65 & 6.65 & 15.61 & 5.18 & 0.44 \\
\hline $\mathbf{S 1 1 2 9 0}$ & $\mathrm{O}=\mathrm{c} 1[\mathrm{n}] \mathrm{ncc} 2 \mathrm{n} 1 \mathrm{ncs} 2$ & .17 & 3.56 & 13.23 & 11.47 & 25.66 & 2.57 & 0.44 \\
\hline S113 & $\mathrm{O}=\mathrm{c} 1 \mathrm{cnsnc} 1$ & .58 & 2.32 & 6.27 & 5.37 & 11.13 & 9.82 & 0.47 \\
\hline S1131 & $\mathrm{O}=\mathrm{c} 1 \mathrm{ncc} 2 \mathrm{c}([\mathrm{nH}] 1) \mathrm{cn}[\mathrm{nH}] 2$ & 1.03 & 0.07 & 9.49 & 8.67 & 21.97 & 19.30 & .47 \\
\hline S1132 & $\mathrm{O}=\mathrm{c} 1 \mathrm{ncc} 2 \mathrm{c}([\mathrm{nH}] 1)[\mathrm{nH}] \mathrm{nc} 2$ & 1.21 & 0.07 & 10.91 & 10.89 & 22.50 & 21.33 & 0.54 \\
\hline S1133 & $\mathrm{n} 2 \mathrm{c}([\mathrm{nH}]$ & .55 & 0.82 & 6.98 & 11.02 & 16.56 & 22.62 & 0.42 \\
\hline S11396 & $\mathrm{O}=\mathrm{c} 1 \mathrm{nnn} 2 \mathrm{c}([\mathrm{nH}] 1) \mathrm{nccn} 2$ & 4.04 & 4.11 & 9.54 & 13.05 & 17.53 & 21.96 & 0.61 \\
\hline S11398 & $\mathrm{O}=\mathrm{c} 1 \mathrm{nnn} 2 \mathrm{c}([\mathrm{nH}] 1) \mathrm{nncn} 2$ & 6.08 & 5.94 & 13.21 & 13.45 & 17.64 & 20.01 & .41 \\
\hline S11399 & $\mathrm{O}=\mathrm{c} 1 \mathrm{n}$ & 6.18 & 5.94 & 13.87 & 14.95 & 17.93 & 22.60 & .40 \\
\hline S114 & & 29 & 2.32 & 8.66 & 7.97 & 19.74 & 19.35 & 0.39 \\
\hline S11594 & 1) $\mathrm{c}[\mathrm{nH}] \mathrm{c} 2$ & -1.19 & -0.99 & 9.65 & 8.02 & 19.10 & 18.64 & 0.46 \\
\hline S11597 & ]1) $\operatorname{coc} 2$ & 1.02 & 0.61 & 6.7 & 7.04 & 13.11 & 14.65 & 0.34 \\
\hline S11600 & $\mathrm{O}=\mathrm{c} 1[\mathrm{nH}$ & 0.52 & -0.08 & 6.52 & 6.29 & 13.14 & 14.11 & 0.34 \\
\hline S11603 & $\mathrm{O}=\mathrm{c} 1[\mathrm{nH}]$ & 0.51 & 0.84 & 9.40 & 7.65 & 16.89 & 15.37 & 0.62 \\
\hline S11604 & $\mathrm{O}=\mathrm{c} 1[\mathrm{nH}] \mathrm{c}(=\mathrm{O}) \mathrm{c}$ & 0.43 & 0.84 & 10.29 & 10.23 & 20.70 & 20.97 & 0.45 \\
\hline S1161 & $\mathrm{O}=\mathrm{c} 1 \mathrm{cnc} 2 \mathrm{c}([\mathrm{nH}] 1)[\mathrm{nH}] \mathrm{nc} 2$ & 0.81 & 0.07 & 10.62 & 10.41 & 17.05 & 18.72 & 0.62 \\
\hline S11613 & $\mathrm{O}=\mathrm{c} 1 \mathrm{cc} 2 \mathrm{nc}[\mathrm{nH}] \mathrm{n} 2 \mathrm{c}(=\mathrm{O})[\mathrm{nH}] 1$ & 1.63 & 1.59 & 12.95 & 10.83 & 24.07 & 18.50 & 0.59 \\
\hline S1162 & $\mathrm{O}=\mathrm{c} 1 \mathrm{cnc} 2 \mathrm{c}([\mathrm{nH}] 1) \mathrm{cn}[\mathrm{nH}] 2$ & 0.67 & 0.07 & 5.42 & 6.89 & 12.94 & 14.67 & 0.45 \\
\hline S11638 & $\mathrm{O}=\mathrm{c} 1 \mathrm{c}$ & 1.90 & 1.97 & 8.35 & 7.97 & 18.00 & 14.69 & 0.47 \\
\hline S1164 & $\mathrm{O}=\mathrm{c}$ & 1.1 & 0.82 & 7.69 & 9.90 & 14.38 & 19.42 & 0.64 \\
\hline S1165 & م-1n-1n & 27 & 1.9 & 14.12 & 14.87 & 23.62 & 25.49 & 0.39 \\
\hline S1166 & H]2 & 24 & 1.90 & 8.66 & 10.60 & 18.73 & 20.28 & 0.61 \\
\hline S11660 & $\mathrm{O}=\mathrm{c} 1 \mathrm{cc} 2 \mathrm{n}$ & 3.89 & 3.80 & 10.57 & 9.41 & 17.17 & 14.35 & 0.84 \\
\hline S11668 & $=c 1 n c n 2 c([n H] 1) c c(=O)[n H] c 2=O$ & 5.72 & 4.57 & 13.25 & 13.63 & 18.56 & 22.70 & 0.63 \\
\hline S11670 & $\mathrm{O}=\mathrm{c} 1[\mathrm{nH}] \mathrm{cc} 2 \mathrm{c}(\mathrm{n} 1) \mathrm{c}(=\mathrm{O})[\mathrm{nH}] \mathrm{c}(=\mathrm{O})[\mathrm{nH}] 2$ & 4.15 & 3.82 & 15.73 & 15.64 & 26.26 & 28.67 & 0.40 \\
\hline S11671 & $\mathrm{O}=\mathrm{c} 1[\mathrm{nH}] \mathrm{cc} 2 \mathrm{c}(\mathrm{n} 1)[\mathrm{nH}] \mathrm{c}(=\mathrm{O})[\mathrm{nH}] \mathrm{c} 2=\mathrm{O}$ & 4.23 & 3.82 & 11.45 & 10.80 & 18.99 & 18.22 & 0.47 \\
\hline
\end{tabular}




\begin{tabular}{|c|c|c|c|c|c|c|c|c|c|}
\hline S1169 & $\mathrm{O}=\mathrm{c} 1[\mathrm{nH}] \mathrm{c}(=\mathrm{O}) \mathrm{c} 2 \mathrm{c}([\mathrm{nH}] 1)[\mathrm{nH}] \mathrm{nc} 2$ & 1.59 & 0.84 & 11.97 & 10.41 & 19.57 & 19.13 & 0.55 & 0.54 \\
\hline S1170 & $\mathrm{O}=\mathrm{c} 1[\mathrm{nH}] \mathrm{c}(=\mathrm{O}) \mathrm{c} 2 \mathrm{c}([\mathrm{nH}] 1) \mathrm{cn}[\mathrm{nH}] 2$ & 1.60 & 0.84 & 6.78 & 7.52 & 12.24 & 14.71 & 0.36 & 0.36 \\
\hline S11714 & $\mathrm{O}=\mathrm{c} 1[\mathrm{nH}] \mathrm{c}(=\mathrm{O}) \mathrm{c} 2 \mathrm{c}([\mathrm{nH}] 1) \mathrm{c}(=\mathrm{O}) \mathrm{cc}[\mathrm{nH}] 2$ & 2.44 & 1.99 & 9.88 & 9.41 & 17.07 & 17.54 & 0.52 & 0.52 \\
\hline S11715 & $\mathrm{O}=\mathrm{c} 1[\mathrm{nH}] \mathrm{c} 2[\mathrm{nH}] \operatorname{ccc}(=\mathrm{O}) \mathrm{c} 2 \mathrm{c}(=\mathrm{O})[\mathrm{nH}] 1$ & 1.65 & 1.99 & 17.00 & 15.26 & 32.44 & 30.08 & 0.55 & .55 \\
\hline S11718 & $\mathrm{O}=\mathrm{c} 1[\mathrm{nH}] \mathrm{c}(=\mathrm{O}) \mathrm{c} 2 \mathrm{c}([\mathrm{nH}] 1) \mathrm{c}(=\mathrm{O}) \mathrm{nc}[\mathrm{nH}] 2$ & 4.35 & 3.82 & 11.62 & 12.32 & 19.64 & 20.73 & 0.45 & .46 \\
\hline S11719 & $\mathrm{O}=\mathrm{c} 1[\mathrm{nH}] \mathrm{c} 2[\mathrm{nH}] \mathrm{cnc}(=\mathrm{O}) \mathrm{c} 2 \mathrm{c}(=\mathrm{O})[\mathrm{nH}] 1$ & 3.51 & 3.82 & 19.49 & 19.17 & 34.56 & 35.43 & 0.47 & 0.49 \\
\hline S1172 & $\mathrm{O}=\mathrm{c} 1[\mathrm{nH}] \mathrm{c}(=\mathrm{O}) \mathrm{n} 2 \mathrm{c}([\mathrm{nH}] 1) \mathrm{ccn} 2$ & 1.78 & 1.59 & 8.73 & 9.43 & 17.12 & 19.20 & 0.51 & .52 \\
\hline S11720 & $\mathrm{O}=\mathrm{c} 1 \mathrm{cc} 2[\mathrm{nH}] \mathrm{cnc}(=\mathrm{O}) \mathrm{n} 2 \mathrm{c}(=\mathrm{O})[\mathrm{nH}] 1$ & 3.78 & 4.57 & 17.01 & 15.68 & 27.39 & 29.80 & .42 & .41 \\
\hline S11722 & $\mathrm{O}=\mathrm{c} 1[\mathrm{nH}] \mathrm{c} 2 \mathrm{nccnc} 2 \mathrm{c}(=\mathrm{O})[\mathrm{nH}] 1$ & 2.66 & 3.05 & 7.33 & 8.52 & 14.83 & 14.70 & 0.48 & .48 \\
\hline S11724 & $\mathrm{O}=\mathrm{c} 1[\mathrm{nH}] \mathrm{c} 2 \mathrm{nncnc} 2 \mathrm{c}(=\mathrm{O})[\mathrm{nH}] 1$ & 4.87 & 4.88 & 11.54 & 10.08 & 15.49 & 14.68 & 0.56 & 0.61 \\
\hline S11725 & $\mathrm{O}=\mathrm{c} 1[\mathrm{nH}] \mathrm{c} 2 \mathrm{ncnnc} 2 \mathrm{c}(=\mathrm{O})[\mathrm{nH}] 1$ & 4.84 & 4.88 & 11.90 & 11.07 & 18.10 & 16.83 & 42 & 43 \\
\hline S11728 & $\mathrm{O}=\mathrm{c} 1[\mathrm{nH}] \mathrm{c}(=\mathrm{O}) \mathrm{c} 2 \mathrm{c}([\mathrm{nH}] 1) \mathrm{c}(=\mathrm{O})[\mathrm{nH}] \mathrm{cn} 2$ & 3.27 & 3.82 & 12.26 & 11.39 & 19.70 & 18.02 & 0.60 & 0.58 \\
\hline S11729 & $\mathrm{O}=\mathrm{c} 1[\mathrm{nH}] \mathrm{c} 2 \mathrm{nc}[\mathrm{nH}] \mathrm{c}(=\mathrm{O}) \mathrm{c} 2 \mathrm{c}(=\mathrm{O})[\mathrm{nH}] 1$ & 2.53 & 3.82 & 12.92 & 10.53 & 23.97 & 18.62 & 0.39 & 0.39 \\
\hline S1173 & $\mathrm{O}=\mathrm{c} 1[\mathrm{nH}] \mathrm{c} 2[\mathrm{nH}] \mathrm{ncc} 2[\mathrm{nH}] \mathrm{c} 1=\mathrm{O}$ & 1.57 & 0.84 & 10.81 & 11.46 & 21.66 & 24.84 & 0.54 & 0.54 \\
\hline S11744 & $\mathrm{O}=\mathrm{c} 1[\mathrm{nH}] \mathrm{c}(=\mathrm{O}) \mathrm{c} 2 \mathrm{c}([\mathrm{nH}] 1) \mathrm{c}(=\mathrm{O}) \operatorname{scn} 2$ & 4.03 & 4.73 & 9.73 & 9.70 & 15.65 & 14.29 & 0.40 & 0.38 \\
\hline S11745 & $\mathrm{O}=\mathrm{c} 1[\mathrm{nH}] \mathrm{c} 2 \mathrm{ncsc}(=\mathrm{O}) \mathrm{c} 2 \mathrm{c}(=\mathrm{O})[\mathrm{nH}] 1$ & & & 10.07 & 10.25 & 19.17 & & & .38 \\
\hline S11788 & $\mathrm{O}=\mathrm{c} 1[\mathrm{nH}] \mathrm{c}(=\mathrm{O}) \mathrm{c} 2 \mathrm{c}([\mathrm{nH}] 1) \mathrm{c}(=\mathrm{O}) \mathrm{cn}[\mathrm{nH}] 2$ & 4.27 & 3.82 & 10.23 & 9.74 & 14.80 & 15.13 & 0.46 & 0.46 \\
\hline S11789 & $\mathrm{O}=\mathrm{c} 1[\mathrm{nH}] \mathrm{c} 2[\mathrm{nH}] \mathrm{ncc}(=\mathrm{O}) \mathrm{c} 2 \mathrm{c}(=\mathrm{O})[\mathrm{nH}] 1$ & 3.45 & 3.82 & 16.11 & 14.60 & 28.14 & 25.51 & 0.55 & 0.56 \\
\hline S1179 & $\mathrm{O}=\mathrm{c} 1[\mathrm{nH}] \mathrm{c} 2[\mathrm{nH}] \mathrm{ncc} 2 \mathrm{oc} 1=\mathrm{O}$ & 2.73 & 2.44 & 12.99 & 14.18 & 21.69 & 25.80 & 0.49 & 0.50 \\
\hline S1180 & $\mathrm{O}=\mathrm{c} 1[\mathrm{nH}] \mathrm{c} 2 \mathrm{cn}[\mathrm{nH}] \mathrm{c} 2 \mathrm{oc} 1=\mathrm{O}$ & 2.64 & 2.44 & 7.80 & 11.08 & 15.49 & 22.74 & 0.50 & 0.55 \\
\hline $\mathbf{S 1 1 8 6 0}$ & $\mathrm{O}=\mathrm{c} 1[\mathrm{nH}] \mathrm{c} 2 \mathrm{c}[\mathrm{nH}] \mathrm{cc} 2[\mathrm{nH}] \mathrm{c} 1=\mathrm{O}$ & -1.10 & -0.99 & 9.67 & 12.21 & 24.33 & 29.90 & 0.43 & 0.43 \\
\hline S11866 & $\mathrm{O}=\mathrm{c} 1[\mathrm{nH}] \mathrm{c} 2 \operatorname{cscc} 2[\mathrm{nH}] \mathrm{c} 1=\mathrm{O}$ & 0.80 & -0.08 & 6.23 & 8.79 & 17.30 & 23.19 & 0.83 & 0.82 \\
\hline S11869 & $\mathrm{O}=\mathrm{c} 1[\mathrm{nH}] \mathrm{c} 2 \mathrm{n}[\mathrm{nH}] \mathrm{cc} 2[\mathrm{nH}] \mathrm{c} 1=\mathrm{O}$ & 0.50 & 0.84 & 9.84 & 12.38 & 22.36 & 27.82 & 0.52 & 0.53 \\
\hline S1191 & $\mathrm{O}=\mathrm{c} 1 \mathrm{cc}[\mathrm{nH}] \mathrm{c} 2 \mathrm{c} 1[\mathrm{nH}] \mathrm{nc} 2$ & -1.71 & -1.76 & 9.65 & 7.13 & 21.36 & 18.69 & 0.51 & 0.48 \\
\hline S1192 & $\mathrm{O}=\mathrm{c} 1 \mathrm{cc}[\mathrm{nH}] \mathrm{c} 2 \mathrm{c} 1 \mathrm{cn}[\mathrm{nH}] 2$ & -1.79 & -1.76 & 12.98 & 11.68 & 27.32 & 25.64 & 0.50 & 0.51 \\
\hline S1193 & $\mathrm{O}=\mathrm{c} 1 \mathrm{cc}[\mathrm{nH}] \mathrm{c} 2 \mathrm{n} 1 \mathrm{ncc} 2$ & -1.65 & -1.01 & 11.46 & 10.40 & 26.39 & 25.15 & 0.50 & 0.49 \\
\hline S1195 & $\mathrm{O}=\mathrm{c} 1 \mathrm{nc}[\mathrm{nH}] \mathrm{c} 2 \mathrm{c} 1[\mathrm{nH}] \mathrm{nc} 2$ & 0.16 & 0.07 & 11.65 & 9.82 & 24.26 & 22.06 & 0.45 & 0.48 \\
\hline S1196 & $\mathrm{O}=\mathrm{c} 1 \mathrm{nc}[\mathrm{nH}] \mathrm{c} 2 \mathrm{c} 1 \mathrm{cn}[\mathrm{nH}] 2$ & 0.05 & 0.07 & 15.28 & 15.11 & 30.08 & 30.16 & 0.43 & 0.43 \\
\hline S11968 & $\mathrm{O}=\mathrm{c} 1[\mathrm{nH}] \mathrm{c} 2 \mathrm{nccnc} 2[\mathrm{nH}] \mathrm{c} 1=\mathrm{O}$ & 3.03 & 3.05 & 8.35 & 10.61 & 17.14 & 22.29 & 0.38 & 0.39 \\
\hline S11970 & $\mathrm{O}=\mathrm{c} 1[\mathrm{nH}] \mathrm{c} 2 \mathrm{nncnc} 2[\mathrm{nH}] \mathrm{c} 1=\mathrm{O}$ & 5.22 & 4.88 & 12.70 & 11.67 & 18.21 & 21.17 & 0.41 & 0.40 \\
\hline S1199 & $\mathrm{c} 1 \mathrm{cnc} 2 \mathrm{c}(\mathrm{n} 1)[\mathrm{nH}] \mathrm{nc} 2$ & -0.99 & -0.70 & 2.81 & 5.23 & 7.16 & 12.28 & 0.45 & 0.46 \\
\hline
\end{tabular}




\begin{tabular}{|c|c|c|c|c|c|c|c|c|c|}
\hline $\mathbf{S 1 2 0 0}$ & $\mathrm{c} 1 \mathrm{cnc} 2 \mathrm{n}(\mathrm{n} 1) \mathrm{ncc} 2$ & -0.72 & 0.05 & 5.48 & 5.51 & 13.15 & 12.63 & 0.55 & 0.57 \\
\hline S1201 & $\mathrm{c} 1 \mathrm{nnc} 2 \mathrm{c}(\mathrm{n} 1) \mathrm{cn}[\mathrm{nH}] 2$ & 1.08 & 1.13 & 5.36 & 6.57 & 10.39 & 12.42 & 0.52 & 0.50 \\
\hline S1202 & $\mathrm{c} 1 \mathrm{nnc} 2 \mathrm{c}(\mathrm{n} 1)[\mathrm{nH}] \mathrm{nc} 2$ & 1.13 & 1.13 & 6.34 & 7.32 & 12.60 & 13.58 & 0.43 & 0.43 \\
\hline S1203 & $\mathrm{c} 1 \mathrm{nnn} 2 \mathrm{c}(\mathrm{n} 1) \mathrm{ccn} 2$ & 1.29 & 1.88 & 4.55 & 7.99 & 9.49 & 14.95 & 0.42 & 0.43 \\
\hline S1205 & $\mathrm{O}=\mathrm{c} 1[\mathrm{nH}] \mathrm{cnc} 2 \mathrm{c} 1[\mathrm{nH}] \mathrm{nc} 2$ & -0.13 & 0.07 & 7.46 & 8.37 & 12.42 & 15.75 & 0.73 & 0.76 \\
\hline S1206 & $\mathrm{O}=\mathrm{c} 1[\mathrm{nH}] \mathrm{cnc} 2 \mathrm{c} 1 \mathrm{cn}[\mathrm{nH}] 2$ & -0.34 & 0.07 & 8.11 & 7.73 & 16.08 & 16.11 & 0.40 & 0.42 \\
\hline S1207 & $\mathrm{O}=\mathrm{c} 1[\mathrm{nH}] \mathrm{cnc} 2 \mathrm{n} 1 \mathrm{ncc} 2$ & -0.22 & 0.82 & 9.67 & 7.54 & 19.20 & 15.83 & 0.79 & 0.76 \\
\hline S12077 & $\mathrm{O}=\mathrm{c} 1 \mathrm{oc}(=\mathrm{O}) \mathrm{c} 2 \mathrm{c}([\mathrm{nH}] 1) \operatorname{coc} 2$ & 2.48 & 2.21 & 9.07 & 10.11 & 17.03 & 18.21 & 0.48 & 0.44 \\
\hline S1208 & $\mathrm{O}=\mathrm{c} 1[\mathrm{nH}] \mathrm{cnn} 2 \mathrm{c} 1 \mathrm{ccn} 2$ & -0.06 & 0.82 & 9.41 & 7.95 & 16.88 & 15.56 & 0.43 & 0.45 \\
\hline $\mathbf{S 1 2 0 8 0}$ & $\mathrm{O}=\mathrm{c} 1 \mathrm{oc}(=\mathrm{O}) \mathrm{c} 2 \mathrm{c}([\mathrm{nH}] 1) \mathrm{csc} 2$ & 1.99 & 1.52 & 8.77 & 9.44 & 17.08 & 17.80 & 0.52 & 0.48 \\
\hline S12087 & $\mathrm{O}=\mathrm{c} 1 \mathrm{oc}(=\mathrm{O}) \mathrm{c} 2 \mathrm{c}([\mathrm{nH}] 1)$ noc 2 & 4.47 & 4.04 & 9.87 & 9.92 & 15.15 & 15.20 & 0.80 & 0.71 \\
\hline S1213 & $\mathrm{O}=\mathrm{c} 1 \mathrm{ocnc} 2 \mathrm{c} 1[\mathrm{nH}] \mathrm{nc} 2$ & 1.47 & 1.67 & 4.54 & 7.10 & 9.26 & 12.84 & 0.42 & 0.40 \\
\hline S1214 & $\mathrm{O}=\mathrm{c} 1 \mathrm{ocnc} 2 \mathrm{c} 1 \mathrm{cn}[\mathrm{nH}] 2$ & 1.26 & 1.67 & 5.76 & 7.59 & 12.94 & 14.31 & 0.39 & 0.36 \\
\hline S1222 & $\mathrm{O}=\mathrm{c} 1 \mathrm{scnc} 2 \mathrm{c} 1 \mathrm{cn}[\mathrm{nH}] 2$ & 0.43 & 0.98 & 5.13 & 6.78 & 11.87 & 13.50 & 0.50 & 0.51 \\
\hline S1223 & $\mathrm{O}=\mathrm{c} 1 \mathrm{scnc} 2 \mathrm{n} 1 \mathrm{ncc} 2$ & 0.45 & 1.73 & 6.26 & 7.15 & 14.53 & 14.08 & 0.74 & 0.75 \\
\hline S1225 & $\mathrm{n} 1 \mathrm{nnc} 2 \mathrm{c}(\mathrm{n} 1)[\mathrm{nH}] \mathrm{nc} 2$ & 3.30 & 2.96 & 9.29 & 9.68 & 16.30 & 15.97 & 0.40 & 0.44 \\
\hline S1226 & $\mathrm{n} 1 \mathrm{nnc} 2 \mathrm{n}(\mathrm{n} 1) \mathrm{ncc} 2$ & 3.45 & 3.71 & 7.69 & 11.02 & 11.67 & 18.31 & 0.42 & 0.42 \\
\hline S1227 & $\mathrm{O}=\mathrm{c} 1[\mathrm{nH}][\mathrm{n}] \mathrm{c} 2 \mathrm{c}([\mathrm{n}] 1)[\mathrm{nH}] \mathrm{nc} 2$ & 1.97 & 1.90 & 6.20 & 7.92 & 16.37 & 14.66 & 0.62 & 0.69 \\
\hline S1228 & $\mathrm{O}=\mathrm{c} 1[\mathrm{nH}][\mathrm{n}] \mathrm{c} 2 \mathrm{c}([\mathrm{n}] 1) \mathrm{cn}[\mathrm{nH}] 2$ & 1.95 & 1.90 & 8.66 & 9.57 & 19.73 & 17.19 & 0.63 & 0.60 \\
\hline S1233 & $\mathrm{O}=\mathrm{c} 1[\mathrm{nH}] \mathrm{nnc} 2 \mathrm{c} 1 \mathrm{cn}[\mathrm{nH}] 2$ & 1.46 & 1.90 & 5.78 & 7.87 & 11.58 & 13.96 & 0.43 & 0.43 \\
\hline S1234 & $\mathrm{O}=\mathrm{c} 1[\mathrm{nH}] \mathrm{nnc} 2 \mathrm{c} 1[\mathrm{nH}] \mathrm{nc} 2$ & 1.72 & 1.90 & 4.93 & 9.25 & 8.25 & 14.76 & 0.47 & 0.47 \\
\hline S12343 & $\mathrm{O}=\mathrm{c} 1[\mathrm{nH}] \mathrm{c} 2 \mathrm{cscc} 2 \mathrm{oc} 1=\mathrm{O}$ & 1.77 & 1.52 & 8.17 & 10.24 & 17.47 & 22.89 & 0.47 & 0.44 \\
\hline S1236 & $\mathrm{O}=\mathrm{c} 1[\mathrm{nH}] \mathrm{nnc} 2 \mathrm{n} 1 \mathrm{ncc} 2$ & 1.61 & 2.65 & 6.17 & 8.35 & 12.71 & 14.66 & 0.37 & 0.42 \\
\hline S124 & n1ncnnc1 & 2.87 & 2.47 & 5.58 & 6.03 & 6.95 & 11.23 & 0.40 & 0.39 \\
\hline S1253 & $\mathrm{O}=\mathrm{c} 1 \mathrm{ccoc} 2 \mathrm{c} 1[\mathrm{nH}] \mathrm{nc} 2$ & -0.27 & -0.16 & 5.10 & 5.71 & 12.68 & 13.64 & 0.62 & 0.57 \\
\hline S1254 & $\mathrm{O}=\mathrm{c} 1 \mathrm{ccoc} 2 \mathrm{c} 1 \mathrm{cn}[\mathrm{nH}] 2$ & -0.42 & -0.16 & 7.34 & 7.16 & 17.70 & 17.53 & 0.62 & 0.64 \\
\hline S1255 & $\mathrm{O}=\mathrm{c} 1 \cos 2 \mathrm{n} 1 \mathrm{ncc} 2$ & -0.27 & 0.59 & 7.53 & 6.97 & 18.67 & 17.58 & 0.39 & 0.38 \\
\hline S1257 & $\mathrm{O}=\mathrm{c} 1 \mathrm{n} \operatorname{coc} 2 \mathrm{c} 1[\mathrm{nH}] \mathrm{nc} 2$ & 1.53 & 1.67 & 6.19 & 7.51 & 14.67 & 15.75 & 0.61 & 0.61 \\
\hline S1258 & $\mathrm{O}=\mathrm{c} 1 \mathrm{n} \operatorname{coc} 2 \mathrm{c} 1 \mathrm{cn}[\mathrm{nH}] 2$ & 1.35 & 1.67 & 9.79 & 9.71 & 20.47 & 20.80 & 0.46 & 0.41 \\
\hline S1261 & $\mathrm{O}=\mathrm{c} 1 \mathrm{ccsc} 2 \mathrm{c} 1 \mathrm{cn}[\mathrm{nH}] 2$ & -1.00 & -0.85 & 7.01 & 6.64 & 17.33 & 17.49 & 0.49 & 0.49 \\
\hline S1264 & $\mathrm{O}=\mathrm{c} 1 \mathrm{ccsc} 2 \mathrm{n} 1 \mathrm{ncc} 2$ & -0.90 & -0.10 & 6.53 & 6.38 & 18.14 & 17.48 & 0.56 & 0.60 \\
\hline
\end{tabular}




\begin{tabular}{|c|c|c|c|c|c|c|c|c|c|}
\hline S1265 & $\mathrm{O}=\mathrm{c} 1 \mathrm{cn}[\mathrm{nH}] \mathrm{c} 2 \mathrm{c} 1[\mathrm{nH}] \mathrm{nc} 2$ & 0.08 & 0.07 & 8.11 & 6.99 & 16.86 & 15.46 & 0.57 & 0.58 \\
\hline S1266 & $\mathrm{O}=\mathrm{c} 1 \mathrm{cn}[\mathrm{nH}] \mathrm{c} 2 \mathrm{c} 1 \mathrm{cn}[\mathrm{nH}] 2$ & -0.02 & 0.07 & 12.00 & 10.79 & 22.94 & 21.25 & 0.47 & 0.47 \\
\hline S1267 & $\mathrm{O}=\mathrm{c} 1 \mathrm{cn}[\mathrm{nH}] \mathrm{c} 2 \mathrm{n} 1 \mathrm{ncc} 2$ & 0.16 & 0.82 & 9.47 & 10.19 & 20.58 & 21.73 & 0.54 & 0.53 \\
\hline S12742 & $\mathrm{O}=\mathrm{c} 1 \mathrm{sc}(=\mathrm{O}) \mathrm{c} 2 \mathrm{c}([\mathrm{nH}] 1) \operatorname{coc} 2$ & 1.51 & 1.52 & 8.38 & 9.10 & 15.02 & 16.74 & 0.61 & 0.57 \\
\hline S1275 & $\mathrm{O}=\mathrm{c} 1 \mathrm{cnsc} 2 \mathrm{n} 1 \mathrm{ncc} 2$ & 0.91 & 1.73 & 5.12 & 7.61 & 13.51 & 15.95 & 0.54 & 0.52 \\
\hline S1278 & $\mathrm{O}=\mathrm{c} 1 \mathrm{n} \csc 2 \mathrm{c} 1 \mathrm{cn}[\mathrm{nH}] 2$ & 0.63 & 0.98 & 9.38 & 9.24 & 20.39 & 20.92 & 0.39 & 0.37 \\
\hline S1281 & $\mathrm{O}=\mathrm{c} 1 \mathrm{nn}[\mathrm{nH}] \mathrm{c} 2 \mathrm{c} 1[\mathrm{nH}] \mathrm{nc} 2$ & 1.92 & 1.90 & 10.72 & 10.70 & 21.11 & 21.07 & 0.38 & 0.37 \\
\hline S1282 & $\mathrm{O}=\mathrm{c} 1 \mathrm{nn}[\mathrm{nH}] \mathrm{c} 2 \mathrm{c} 1 \mathrm{cn}[\mathrm{nH}] 2$ & 1.73 & 1.90 & 14.57 & 15.25 & 26.49 & 28.02 & 0.53 & 0.50 \\
\hline $\mathbf{S 1 2 8 6 0}$ & $\mathrm{O}=\mathrm{c} 1 \mathrm{sc}(=\mathrm{O}) \mathrm{c} 2 \mathrm{c}([\mathrm{nH}] 1) \mathrm{nccn} 2$ & 3.02 & 3.96 & 8.54 & 10.93 & 15.03 & 17.05 & 0.45 & 0.43 \\
\hline $\mathbf{S 1 2 8 6 2}$ & $\mathrm{O}=\mathrm{c} 1 \mathrm{sc}(=\mathrm{O}) \mathrm{c} 2 \mathrm{c}([\mathrm{nH}] 1) \mathrm{nncn} 2$ & 5.18 & 5.79 & 12.20 & 11.70 & 15.25 & 15.97 & 0.41 & 0.36 \\
\hline S12863 & $\mathrm{O}=\mathrm{c} 1 \mathrm{sc}(=\mathrm{O}) \mathrm{c} 2 \mathrm{c}([\mathrm{nH}] 1) \mathrm{ncnn} 2$ & 5.16 & 5.79 & 12.68 & 13.08 & 17.86 & 18.63 & 0.44 & 0.40 \\
\hline S1297 & $\mathrm{O}=\mathrm{c} 1 \mathrm{oc} 2 \mathrm{c} \operatorname{cnn} 2 \mathrm{c}(=\mathrm{O})[\mathrm{nH}] 1$ & 2.96 & 3.19 & 9.24 & 8.69 & 14.80 & 15.64 & 0.52 & 0.48 \\
\hline S1299 & $\mathrm{O}=\mathrm{c} 1 \mathrm{sc} 2 \mathrm{cn}[\mathrm{nH}] \mathrm{c} 2 \mathrm{c}(=\mathrm{O})[\mathrm{nH}] 1$ & 2.02 & 1.75 & 5.69 & 7.90 & 10.44 & 12.76 & 0.49 & 0.54 \\
\hline $\mathbf{S 1 3 0 0}$ & $\mathrm{O}=\mathrm{c} 1[\mathrm{nH}] \mathrm{c}(=\mathrm{O}) \mathrm{c} 2 \mathrm{c}(\mathrm{s} 1)[\mathrm{nH}] \mathrm{nc} 2$ & 1.86 & 1.75 & 8.81 & 7.89 & 14.05 & 14.50 & 0.48 & 0.48 \\
\hline S1312 & $\mathrm{O}=\mathrm{c} 1 \mathrm{oc}(=\mathrm{O}) \mathrm{c} 2 \mathrm{c}(\mathrm{o} 1)[\mathrm{nH}] \mathrm{nc} 2$ & 4.19 & 4.04 & 10.93 & 10.68 & 15.80 & 17.29 & 0.79 & 0.80 \\
\hline $\mathbf{S 1 3 2 5 6}$ & $\mathrm{O}=\mathrm{c} 1 \mathrm{ncc} 2 \mathrm{n} 1 \mathrm{cc}[\mathrm{nH}] \mathrm{c} 2$ & -1.04 & -1.01 & 12.55 & 9.77 & 29.57 & 23.32 & 0.56 & 0.52 \\
\hline S13259 & $\mathrm{O}=\mathrm{c} 1 \mathrm{cnc} 2 \mathrm{n} 1 \mathrm{cc}[\mathrm{nH}] \mathrm{c} 2$ & -1.49 & -1.01 & 10.99 & 7.18 & 24.30 & 17.02 & 0.42 & 0.42 \\
\hline S1335 & {$[\mathrm{nH}] 1 \mathrm{cc} 2 \mathrm{c}(\mathrm{c} 1) \mathrm{cno} 2$} & -3.42 & -3.14 & 7.12 & 5.91 & 18.63 & 16.05 & 0.50 & 0.51 \\
\hline S1336 & o1cc2c(c1)cno2 & -1.33 & -1.54 & 3.87 & 3.76 & 11.21 & 11.26 & 0.50 & 0.49 \\
\hline S13367 & $\mathrm{O}=\mathrm{c} 1 \mathrm{ccc} 2 \mathrm{n} 1 \mathrm{ccoc} 2$ & -1.39 & -1.24 & 5.13 & 5.26 & 16.33 & 14.97 & 0.45 & 0.43 \\
\hline S1337 & $\mathrm{s} 1 \mathrm{cc} 2 \mathrm{c}(\mathrm{c} 1) \mathrm{cno} 2$ & -1.84 & -2.23 & 3.64 & 3.08 & 11.45 & 10.81 & 0.65 & 0.65 \\
\hline S1338 & $\mathrm{n} 1 \mathrm{cc} 2 \mathrm{c}(\mathrm{o} 1) \mathrm{n}[\mathrm{nH}] \mathrm{c} 2$ & -1.40 & -1.31 & 7.84 & 6.93 & 17.38 & 15.41 & 0.63 & 0.73 \\
\hline S1339 & $\mathrm{n} 1 \mathrm{cc} 2 \mathrm{c}(\mathrm{o} 1) \mathrm{c}[\mathrm{nH}] \mathrm{n} 2$ & -1.66 & -1.31 & 7.10 & 6.26 & 15.37 & 14.76 & 0.47 & 0.45 \\
\hline S1340 & $\mathrm{n} 1 \mathrm{cc} 2 \mathrm{c}(\mathrm{o} 1) \mathrm{onc} 2$ & 1.22 & 0.29 & 6.59 & 5.20 & 10.71 & 10.83 & 0.43 & 0.44 \\
\hline S1342 & n1cc2c(o1)noc2 & 1.02 & 0.29 & 8.21 & 5.73 & 14.85 & 11.96 & 0.55 & 0.51 \\
\hline S1344 & $\mathrm{n} 1 \mathrm{oc} 2 \mathrm{c}(\mathrm{c} 1) \mathrm{cns} 2$ & 0.27 & -0.40 & 5.18 & 4.38 & 9.84 & 10.06 & 0.59 & 0.59 \\
\hline S1346 & $\mathrm{n} 1 \mathrm{cc} 2 \mathrm{c}(\mathrm{o} 1) \mathrm{nsc} 2$ & 0.17 & -0.40 & 7.28 & 4.99 & 14.13 & 11.35 & 0.59 & 0.59 \\
\hline S1348 & c1nc2c([nH]1)onc2 & -1.18 & -1.31 & 9.04 & 7.14 & 17.03 & 16.55 & 0.49 & 0.62 \\
\hline S1349 & $\mathrm{c} 1 \mathrm{nc} 2 \mathrm{c}([\mathrm{nH}] 1) \mathrm{cno} 2$ & -1.00 & -1.31 & 10.51 & 8.56 & 20.43 & 17.72 & 0.47 & 0.44 \\
\hline $\mathbf{S 1 3 5 0}$ & c1nc2c(o1)onc2 & 0.75 & 0.29 & 5.16 & 4.75 & 10.77 & 10.91 & 0.46 & 0.49 \\
\hline S13507 & $\mathrm{O}=\mathrm{c} 1 \mathrm{cc}[\mathrm{nH}] \mathrm{c} 2 \mathrm{c} 1 \mathrm{c}[\mathrm{nH}] \mathrm{c} 2$ & -4.56 & -3.59 & 6.47 & 8.49 & 23.26 & 22.78 & 0.76 & 0.71 \\
\hline
\end{tabular}




\begin{tabular}{|c|c|c|c|c|c|c|c|c|c|}
\hline S1351 & c1oc2c(n1)onc2 & 0.76 & 0.29 & 5.83 & 5.16 & 12.35 & 11.45 & 0.64 & 0.63 \\
\hline $\mathbf{S 1 3 5 1 0}$ & $\mathrm{O}=\mathrm{c} 1 \mathrm{cc}[\mathrm{nH}] \mathrm{c} 2 \mathrm{c} 1 \operatorname{coc} 2$ & -2.33 & -1.99 & 8.96 & 7.49 & 22.18 & 19.49 & 0.61 & 0.59 \\
\hline S13513 & $\mathrm{O}=\mathrm{c} 1 \mathrm{cc}[\mathrm{nH}] \mathrm{c} 2 \mathrm{c} 1 \mathrm{csc} 2$ & -2.80 & -2.68 & 8.65 & 6.74 & 22.12 & 18.86 & 0.36 & 0.37 \\
\hline S13516 & $\mathrm{O}=\mathrm{c} 1 \mathrm{cc}[\mathrm{nH}] \mathrm{c} 2 \mathrm{c} 1 \mathrm{n}[\mathrm{nH}] \mathrm{c} 2$ & -2.93 & -1.76 & 9.54 & 11.43 & 27.11 & 26.96 & 0.41 & 0.35 \\
\hline S13517 & $\mathrm{O}=\mathrm{c} 1 \mathrm{cc}[\mathrm{nH}] \mathrm{c} 2 \mathrm{c} 1 \mathrm{c}[\mathrm{nH}] \mathrm{n} 2$ & -2.82 & -1.76 & 5.73 & 7.36 & 19.26 & 18.14 & 0.35 & 0.33 \\
\hline S1352 & c1nc2c(s1)onc2 & -0.17 & -0.40 & 4.71 & 4.09 & 10.91 & 10.57 & 0.39 & 0.37 \\
\hline S13521 & $\mathrm{O}=\mathrm{c} 1 \mathrm{cc}[\mathrm{nH}] \mathrm{c} 2 \mathrm{c} 1 \operatorname{con} 2$ & -0.25 & -0.16 & 8.58 & 7.32 & 18.01 & 16.19 & 0.44 & 0.43 \\
\hline $\mathbf{S 1 3 5 2 6}$ & $\mathrm{O}=\mathrm{c} 1 \mathrm{ccn} 2 \mathrm{c}(\mathrm{c} 1) \mathrm{nc}[\mathrm{nH}] 2$ & -0.86 & -1.01 & 16.08 & 11.49 & 34.21 & 24.95 & 0.46 & 0.51 \\
\hline S1353 & $\mathrm{c} 1 \mathrm{nc} 2 \mathrm{c}(\mathrm{s} 1) \mathrm{cno} 2$ & 0.00 & -0.40 & 5.00 & 4.57 & 12.16 & 11.18 & 0.63 & 0.61 \\
\hline $\mathbf{S 1 3 5 3 4}$ & $\mathrm{O}=\mathrm{c} 1 \mathrm{cc}[\mathrm{nH}] \mathrm{c} 2 \mathrm{c} 1 \mathrm{n}[\mathrm{nH}] \mathrm{n} 2$ & -1.14 & 0.07 & 8.68 & 9.28 & 22.65 & 20.07 & 0.62 & 0.65 \\
\hline S1354 & $\mathrm{n} 1 \mathrm{cc} 2 \mathrm{c}(\mathrm{o} 1)[\mathrm{nH}] \mathrm{nn} 2$ & 0.87 & 0.52 & 8.12 & 7.92 & 13.95 & 15.93 & 0.52 & 0.49 \\
\hline S13540 & $\mathrm{O}=\mathrm{c} 1 \mathrm{cc}[\mathrm{nH}] \mathrm{c} 2 \mathrm{c} 1$ non 2 & 1.76 & 1.67 & 12.38 & 10.19 & 22.37 & 19.46 & 0.42 & 0.41 \\
\hline S13541 & $\mathrm{O}=\mathrm{c} 1 \mathrm{cc}[\mathrm{nH}] \mathrm{c} 2 \mathrm{c} 1 \mathrm{nsn} 2$ & 0.39 & 0.98 & 10.11 & 9.32 & 22.27 & 18.50 & 0.51 & 0.49 \\
\hline S13547 & $\mathrm{O}=\mathrm{c} 1 \operatorname{ccn} 2 \mathrm{c}(\mathrm{c} 1) \operatorname{ccc} 2$ & -1.16 & -2.46 & 7.86 & 5.40 & 23.89 & 16.86 & 0.49 & 0.45 \\
\hline S13548 & $\mathrm{O}=\mathrm{c} 1 \mathrm{ccn} 2 \mathrm{c}(\mathrm{c} 1) \mathrm{cnc} 2$ & 0.78 & -0.63 & 9.66 & 7.17 & 22.64 & 17.24 & 0.82 & 0.79 \\
\hline S13549 & $\mathrm{O}=\mathrm{c} 1 \mathrm{ccn} 2 \mathrm{c}(\mathrm{c} 1) \mathrm{ccnc} 2$ & 0.67 & -0.63 & 8.65 & 6.67 & 21.45 & 16.14 & 0.40 & 0.36 \\
\hline S1355 & $\mathrm{n} 1 \mathrm{oc} 2 \mathrm{c}(\mathrm{c} 1)[\mathrm{nH}] \mathrm{nn} 2$ & 1.08 & 0.52 & 10.66 & 9.34 & 17.91 & 17.10 & 0.44 & 0.48 \\
\hline S13558 & $\mathrm{O}=\mathrm{c} 1 \mathrm{ccn} 2 \mathrm{c}(\mathrm{c} 1)[\mathrm{nH}] \mathrm{c}(=\mathrm{O}) \mathrm{cc} 2$ & 2.06 & 0.14 & 11.43 & 8.30 & 22.95 & 18.87 & 0.30 & 0.31 \\
\hline S13559 & $\mathrm{O}=\mathrm{c} 1[\mathrm{nH}] \mathrm{cc} 2 \mathrm{c}(\mathrm{c} 1) \mathrm{c}(=\mathrm{O}) \mathrm{cc}[\mathrm{nH}] 2$ & -0.33 & -0.61 & 13.28 & 12.04 & 25.61 & 27.18 & 0.34 & 0.31 \\
\hline S1356 & {$[\mathrm{nH}] 1 \mathrm{nc} 2 \mathrm{c}(\mathrm{n} 1) \mathrm{cno} 2$} & 0.34 & 0.52 & 6.34 & 6.25 & 12.13 & 11.87 & 0.57 & 0.54 \\
\hline $\mathbf{S 1 3 5 6 0}$ & $\mathrm{O}=\mathrm{c} 1[\mathrm{nH}] \mathrm{cc} 2 \mathrm{c}(\mathrm{c} 1)[\mathrm{nH}] \mathrm{ccc} 2=\mathrm{O}$ & -0.04 & -0.61 & 9.62 & 8.50 & 19.00 & 19.61 & 0.43 & 0.41 \\
\hline $\mathbf{S} 13562$ & $\mathrm{O}=\mathrm{c} 1 \mathrm{ccn} 2 \mathrm{c}(\mathrm{c} 1) \cos 2=\mathrm{O}$ & 2.07 & 1.74 & 8.91 & 8.55 & 19.40 & 17.62 & 0.54 & 0.57 \\
\hline S13568 & $\mathrm{O}=\mathrm{c} 1 \mathrm{ccn} 2 \mathrm{c}(\mathrm{c} 1) \operatorname{css} 2=\mathrm{O}$ & 1.42 & 1.05 & 8.25 & 7.72 & 19.04 & 16.78 & 0.52 & 0.53 \\
\hline S13570 & $\mathrm{O}=\mathrm{c} 1 \mathrm{ccn} 2 \mathrm{c}(\mathrm{c} 1) \operatorname{sc}(=\mathrm{O}) \mathrm{cc} 2$ & 2.34 & 1.05 & 12.44 & 9.11 & 22.61 & 18.91 & 0.45 & 0.46 \\
\hline S13579 & $\mathrm{O}=\mathrm{c} 1[\mathrm{nH}] \mathrm{nc} 2 \mathrm{c}(\mathrm{c} 1) \mathrm{c}(=\mathrm{O}) \mathrm{cc}[\mathrm{nH}] 2$ & 1.08 & 1.22 & 10.95 & 9.74 & 20.81 & 19.91 & 0.42 & 0.44 \\
\hline S13580 & $\mathrm{O}=\mathrm{c} 1[\mathrm{nH}] \mathrm{nc} 2 \mathrm{c}(\mathrm{c} 1)[\mathrm{nH}] \mathrm{ccc} 2=\mathrm{O}$ & 1.26 & 1.22 & 12.41 & 10.44 & 22.58 & 21.53 & 0.45 & 0.46 \\
\hline S1361 & $\mathrm{O}=\mathrm{c} 1 \mathrm{c}(=\mathrm{O}) \mathrm{c} 2 \mathrm{c}(\mathrm{c} 1=\mathrm{O}) \mathrm{onc} 2$ & 6.73 & 7.14 & 14.45 & 11.40 & 17.22 & 18.46 & 0.52 & 0.55 \\
\hline S1362 & o1nc $2 \mathrm{c}(\mathrm{n} 1) \mathrm{cno} 2$ & 3.04 & 2.12 & 8.15 & 6.01 & 11.01 & 9.76 & 0.65 & 0.63 \\
\hline S13624 & $\mathrm{O}=\mathrm{c} 1 \mathrm{cc}[\mathrm{nH}] \mathrm{c} 2 \mathrm{c} 1 \mathrm{nccn} 2$ & -0.59 & 0.45 & 8.49 & 8.97 & 22.52 & 19.35 & 0.52 & 0.52 \\
\hline S13626 & $\mathrm{O}=\mathrm{c} 1 \mathrm{cc}[\mathrm{nH}] \mathrm{c} 2 \mathrm{c} 1 \mathrm{nn} \mathrm{cn} 2$ & 1.59 & 2.28 & 12.04 & 11.81 & 24.42 & 22.71 & 0.60 & 0.59 \\
\hline S13627 & $\mathrm{O}=\mathrm{c} 1 \mathrm{cc}[\mathrm{nH}] \mathrm{c} 2 \mathrm{c} 1 \mathrm{ncnn} 2$ & 1.62 & 2.28 & 10.81 & 10.22 & 21.28 & 19.26 & 0.47 & 0.43 \\
\hline
\end{tabular}




\begin{tabular}{|c|c|c|c|c|c|c|c|c|c|}
\hline S1363 & $\mathrm{s} 1 \mathrm{nc} 2 \mathrm{c}(\mathrm{n} 1) \mathrm{cno} 2$ & 1.91 & 1.43 & 4.84 & 5.21 & 8.26 & 8.98 & 0.32 & 0.34 \\
\hline S13630 & $\mathrm{O}=\mathrm{c} 1 \mathrm{cc}[\mathrm{nH}] \mathrm{c} 2 \mathrm{c} 1 \mathrm{c}(=\mathrm{O})[\mathrm{nH}] \mathrm{cn} 2$ & -0.81 & 1.22 & 9.20 & 10.74 & 26.44 & 22.99 & 0.43 & 0.45 \\
\hline S13631 & $\mathrm{O}=\mathrm{c} 1 \mathrm{cc}[\mathrm{nH}] \mathrm{c} 2 \mathrm{c} 1 \mathrm{nc}[\mathrm{nH}] \mathrm{c} 2=\mathrm{O}$ & 0.27 & 1.22 & 8.98 & 12.10 & 23.15 & 22.03 & 0.40 & 0.42 \\
\hline S13635 & $\mathrm{O}=\mathrm{c} 1 \mathrm{cnc} 2 \mathrm{c}(\mathrm{o} 1)[\mathrm{nH}] \mathrm{ccc} 2=\mathrm{O}$ & 2.26 & 2.82 & 13.03 & 11.94 & 24.93 & 23.28 & 0.52 & 0.51 \\
\hline S13689 & $\mathrm{O}=\mathrm{c} 1 \operatorname{ccn} 2 \mathrm{c}(\mathrm{c} 1) \operatorname{scc} 2=\mathrm{O}$ & 1.33 & 1.05 & 9.39 & 7.37 & 21.01 & 16.59 & 0.30 & 0.30 \\
\hline S1369 & $\mathrm{c} 1 \mathrm{ccc} 2 \mathrm{c}(\mathrm{c} 1) \mathrm{onc} 2$ & -2.18 & -2.76 & 2.62 & 3.33 & 10.74 & 11.82 & 0.47 & 0.48 \\
\hline S1370 & c1ncc2c(c1)onc2 & -0.23 & -0.93 & 4.13 & 4.21 & 9.18 & 11.10 & 0.34 & 0.34 \\
\hline S1371 & $\mathrm{c} 1 \mathrm{ncc} 2 \mathrm{c}(\mathrm{c} 1) \mathrm{cno} 2$ & -0.23 & -0.93 & 5.05 & 4.47 & 10.02 & 11.35 & 0.47 & 0.38 \\
\hline S1372 & $\mathrm{c} 1 \mathrm{cnc} 2 \mathrm{c}(\mathrm{c} 1) \mathrm{cno} 2$ & -0.39 & -0.93 & 6.14 & 5.37 & 14.13 & 12.89 & 0.38 & 0.41 \\
\hline S1373 & $\mathrm{c} 1 \mathrm{cnc} 2 \mathrm{c}(\mathrm{c} 1) \mathrm{onc} 2$ & -0.65 & -0.93 & 4.44 & 4.65 & 10.78 & 12.18 & 0.49 & 0.45 \\
\hline S1375 & c1nnc2c(c1)cno2 & 1.70 & 0.90 & 9.27 & 7.54 & 17.02 & 14.66 & 0.55 & 0.53 \\
\hline S1376 & $\mathrm{n} 1 \mathrm{ncc} 2 \mathrm{c}(\mathrm{c} 1) \mathrm{onc} 2$ & 1.91 & 0.90 & 7.65 & 6.38 & 14.49 & 12.88 & 0.65 & 0.71 \\
\hline S13769 & $\mathrm{O}=\mathrm{c} 1[\mathrm{n}] \mathrm{nc} 2 \mathrm{n} 1 \mathrm{ccsc} 2$ & 2.08 & 1.73 & 7.58 & 8.79 & 17.26 & 17.16 & 0.33 & 0.31 \\
\hline S1377 & $\mathrm{O}=\mathrm{c} 1[\mathrm{nH}] \operatorname{ccc} 2 \mathrm{c} 1 \mathrm{cno} 2$ & 0.14 & -0.16 & 8.92 & 7.34 & 16.29 & 16.10 & 0.62 & 0.59 \\
\hline S1380 & $\mathrm{O}=\mathrm{c} 1 \mathrm{ccc} 2 \mathrm{c}([\mathrm{nH}] 1) \mathrm{cno} 2$ & 1.12 & -0.16 & 6.91 & 6.37 & 14.65 & 13.73 & 0.51 & 0.47 \\
\hline S1384 & $\mathrm{O}=\mathrm{c} 1$ occc $2 \mathrm{c} 1 \mathrm{onc} 2$ & 1.76 & 1.44 & 8.29 & 7.81 & 17.00 & 15.54 & 0.43 & 0.41 \\
\hline S1395 & c1ncc $2 \mathrm{c}(\mathrm{n} 1) \mathrm{cno} 2$ & 1.32 & 0.90 & 3.75 & 5.21 & 6.77 & 10.42 & 0.51 & 0.47 \\
\hline S1396 & c1ncc $2 \mathrm{c}(\mathrm{n} 1)$ onc 2 & 1.58 & 0.90 & 7.22 & 5.66 & 11.87 & 10.86 & 0.67 & 0.65 \\
\hline S1398 & $\mathrm{n} 1 \mathrm{nnc} 2 \mathrm{c}(\mathrm{c} 1) \mathrm{cno} 2$ & 3.77 & 2.73 & 11.45 & 8.85 & 18.02 & 14.89 & 0.57 & 0.48 \\
\hline S1399 & $\mathrm{O}=\mathrm{c} 1[\mathrm{nH}] \mathrm{ncc} 2 \mathrm{c} 1 \mathrm{cno} 2$ & 1.97 & 1.67 & 5.57 & 6.69 & 11.08 & 12.04 & 0.48 & 0.51 \\
\hline S1400 & $\mathrm{O}=\mathrm{c} 1[\mathrm{nH}] \mathrm{ncc} 2 \mathrm{c} 1 \mathrm{onc} 2$ & 2.03 & 1.67 & 8.11 & 6.93 & 16.11 & 12.57 & 0.46 & 0.42 \\
\hline S1403 & $\mathrm{O}=\mathrm{c} 1 \mathrm{ncc} 2 \mathrm{c}([\mathrm{nH}] 1) \mathrm{cno} 2$ & 2.96 & 1.67 & 10.27 & 9.29 & 19.97 & 17.43 & 0.34 & 0.35 \\
\hline S142 & $\mathrm{O}=\mathrm{c} 1 \mathrm{cnscn} 1$ & 2.43 & 2.32 & 7.68 & 6.24 & 15.82 & 13.64 & 0.36 & 0.33 \\
\hline S1428 & $\mathrm{O}=\mathrm{c} 1[\mathrm{nH}] \mathrm{c}(=\mathrm{O}) \mathrm{c} 2 \mathrm{c}([\mathrm{nH}] 1) \mathrm{cno} 2$ & 3.45 & 2.44 & 11.42 & 9.42 & 17.73 & 15.43 & 0.29 & 0.29 \\
\hline S1439 & $\mathrm{O}=\mathrm{c} 1 \mathrm{cc}[\mathrm{nH}] \mathrm{c} 2 \mathrm{c} 1 \mathrm{cno} 2$ & 0.08 & -0.16 & 11.33 & 8.56 & 21.92 & 19.65 & 0.57 & 0.55 \\
\hline S14395 & $\mathrm{O}=\mathrm{c} 1 \mathrm{ccn} 2 \mathrm{c}(\mathrm{n} 1)[\mathrm{nH}] \mathrm{cc} 2$ & -0.83 & -1.01 & 9.12 & 8.22 & 27.27 & 21.28 & 0.57 & 0.56 \\
\hline S14397 & $\mathrm{O}=\mathrm{c} 1 \mathrm{nc}[\mathrm{nH}] \mathrm{c} 2 \mathrm{c} 1 \mathrm{c}[\mathrm{nH}] \mathrm{c} 2$ & -2.62 & -1.76 & 8.62 & 12.49 & 26.31 & 28.53 & 0.40 & 0.37 \\
\hline S14400 & $\mathrm{O}=\mathrm{c} 1 \mathrm{ccn} 2 \mathrm{c}(\mathrm{n} 1) \mathrm{occ} 2$ & 0.92 & 0.59 & 11.90 & 9.82 & 28.02 & 21.35 & 0.57 & 0.64 \\
\hline $\mathrm{S} 14402$ & $\mathrm{O}=\mathrm{c} 1 \mathrm{nc}[\mathrm{nH}] \mathrm{c} 2 \mathrm{c} 1 \operatorname{coc} 2$ & -0.44 & -0.16 & 11.13 & 10.68 & 25.22 & 24.10 & 0.50 & 0.51 \\
\hline S14407 & $\mathrm{O}=\mathrm{c} 1 \mathrm{nc}[\mathrm{nH}] \mathrm{c} 2 \mathrm{c} 1 \mathrm{csc} 2$ & -0.93 & -0.85 & 10.77 & 9.98 & 25.16 & 23.61 & 0.50 & 0.50 \\
\hline S1441 & $\mathrm{O}=\mathrm{c} 1 \mathrm{nc}[\mathrm{nH}] \mathrm{c} 2 \mathrm{c} 1 \mathrm{cno} 2$ & 1.92 & 1.67 & 13.54 & 11.22 & 24.77 & 23.10 & 0.53 & 0.55 \\
\hline
\end{tabular}




\begin{tabular}{|c|c|c|c|c|c|c|c|c|c|}
\hline S14410 & $\mathrm{O}=\mathrm{c} 1 \mathrm{ccn} 2 \mathrm{c}(\mathrm{n} 1)[\mathrm{nH}] \mathrm{nc} 2$ & 1.23 & 0.82 & 11.41 & 9.01 & 26.39 & 19.82 & 0.45 & 0.43 \\
\hline S14412 & $\mathrm{O}=\mathrm{c} 1 \mathrm{nc}[\mathrm{nH}] \mathrm{c} 2 \mathrm{c} 1 \mathrm{n}[\mathrm{nH}] \mathrm{c} 2$ & -1.00 & 0.07 & 11.78 & 15.59 & 29.33 & 33.06 & 0.39 & 0.38 \\
\hline S14413 & $\mathrm{O}=\mathrm{c} 1 \mathrm{nc}[\mathrm{nH}] \mathrm{c} 2 \mathrm{c} 1 \mathrm{c}[\mathrm{nH}] \mathrm{n} 2$ & -0.91 & 0.07 & 7.92 & 10.86 & 22.49 & 22.77 & 0.58 & 0.61 \\
\hline S14419 & $\mathrm{O}=\mathrm{c} 1 \mathrm{nc}[\mathrm{nH}] \mathrm{c} 2 \mathrm{c} 1 \mathrm{con} 2$ & 1.62 & 1.67 & 10.77 & 10.00 & 21.20 & 19.68 & 0.53 & 0.52 \\
\hline S1442 & $\mathrm{c} 1 \mathrm{cnc} 2 \mathrm{c}(\mathrm{n} 1) \mathrm{onc} 2$ & 1.12 & 0.90 & 5.20 & 5.67 & 10.47 & 11.00 & 0.69 & 0.62 \\
\hline S14422 & $\mathrm{O}=\mathrm{c} 1 \mathrm{ccn} 2 \mathrm{c}(\mathrm{n} 1) \mathrm{snc} 2$ & 2.42 & 1.73 & 13.08 & 10.59 & 25.81 & 20.02 & 0.46 & 0.45 \\
\hline S14426 & $\mathrm{O}=\mathrm{c} 1 \mathrm{ccn} 2 \mathrm{c}(\mathrm{n} 1) \mathrm{nc}[\mathrm{nH}] 2$ & 0.87 & 0.82 & 18.19 & 16.43 & 37.10 & 32.79 & 0.37 & 0.42 \\
\hline S14436 & $\mathrm{O}=\mathrm{c} 1 \mathrm{ccn} 2 \mathrm{c}(\mathrm{n} 1) \operatorname{sen} 2$ & 1.38 & 1.73 & 9.64 & 8.61 & 23.59 & 17.20 & 0.62 & 0.64 \\
\hline S14442 & $\mathrm{O}=\mathrm{c} 1 \mathrm{nc}[\mathrm{nH}] \mathrm{c} 2 \mathrm{c} 1 \mathrm{n}[\mathrm{nH}] \mathrm{n} 2$ & 0.77 & 1.90 & 10.84 & 12.93 & 24.91 & 25.05 & 0.63 & 0.63 \\
\hline S1445 & $\mathrm{O}=\mathrm{c} 1[\mathrm{nH}] \mathrm{cnc} 2 \mathrm{c} 1 \mathrm{onc} 2$ & 1.69 & 1.67 & 10.15 & 8.70 & 17.12 & 15.11 & 0.42 & 0.47 \\
\hline S14453 & $\mathrm{O}=\mathrm{c} 1 \mathrm{nc}[\mathrm{nH}] \mathrm{c} 2 \mathrm{c} 1 \mathrm{non} 2$ & 3.60 & 3.50 & 14.70 & 13.03 & 24.43 & 23.30 & 0.54 & 0.61 \\
\hline S14454 & $\mathrm{O}=\mathrm{c} 1 \mathrm{nc}[\mathrm{nH}] \mathrm{c} 2 \mathrm{c} 1 \mathrm{nsn} 2$ & 2.29 & 2.81 & 12.23 & 12.21 & 24.41 & 22.48 & 0.38 & 0.38 \\
\hline S1446 & $\mathrm{O}=\mathrm{c} 1[\mathrm{nH}] \mathrm{cnc} 2 \mathrm{c} 1 \mathrm{cno} 2$ & 1.87 & 1.67 & 10.92 & 8.92 & 17.29 & 15.03 & 0.41 & 0.46 \\
\hline S14465 & $\mathrm{O}=\mathrm{c} 1 \mathrm{ccn} 2 \mathrm{c}(\mathrm{n} 1) \operatorname{cccc} 2$ & 0.07 & -0.63 & 10.10 & 8.41 & 27.00 & 21.03 & 0.59 & 0.62 \\
\hline S14469 & $\mathrm{O}=\mathrm{c} 1 \mathrm{ccn} 2 \mathrm{c}(\mathrm{n} 1) \mathrm{ccnc} 2$ & 1.93 & 1.20 & 11.33 & 9.35 & 25.05 & 19.57 & 0.63 & 0.62 \\
\hline S14471 & $\mathrm{O}=\mathrm{c} 1 \mathrm{ccn} 2 \mathrm{c}(\mathrm{n} 1) \operatorname{ccc} 2$ & 0.92 & 1.20 & 9.61 & 8.43 & 25.00 & 18.33 & 0.70 & 0.67 \\
\hline S14473 & $\mathrm{O}=\mathrm{c} 1 \mathrm{ccn} 2 \mathrm{c}(\mathrm{n} 1) \mathrm{nccc} 2$ & 1.51 & 1.20 & 13.39 & 11.87 & 30.30 & 25.74 & 0.71 & 0.65 \\
\hline S1448 & $\mathrm{O}=\mathrm{c} 1 \mathrm{cnc} 2 \mathrm{c}(\mathrm{o} 1) \mathrm{cno} 2$ & 3.91 & 3.27 & 9.08 & 7.61 & 12.23 & 12.04 & 0.57 & 0.55 \\
\hline S14481 & $\mathrm{O}=\mathrm{c} 1 \mathrm{ccn} 2 \mathrm{c}(\mathrm{n} 1) \mathrm{c}(=\mathrm{O})[\mathrm{nH}] \mathrm{cc} 2$ & 1.52 & 1.97 & 11.98 & 13.35 & 28.40 & 29.88 & 0.29 & 0.30 \\
\hline S14482 & $\mathrm{O}=\mathrm{c} 1 \mathrm{ncn} 2 \mathrm{c}(\mathrm{c} 1) \mathrm{c}(=\mathrm{O})[\mathrm{nH}] \mathrm{cc} 2$ & 2.29 & 1.97 & 12.66 & 12.78 & 27.46 & 26.94 & 0.38 & 0.38 \\
\hline S14483 & $\mathrm{O}=\mathrm{c} 1 \mathrm{ccn} 2 \mathrm{c}(\mathrm{n} 1) \mathrm{cc}[\mathrm{nH}] \mathrm{c} 2=\mathrm{O}$ & 1.63 & 1.97 & 12.90 & 10.93 & 27.30 & 21.60 & 0.45 & 0.45 \\
\hline S14487 & $\mathrm{O}=\mathrm{c} 1 \mathrm{ccn} 2 \mathrm{c}(\mathrm{n} 1)[\mathrm{nH}] \mathrm{c}(=\mathrm{O}) \mathrm{cc} 2$ & 3.20 & 1.97 & 13.79 & 10.45 & 25.98 & 22.22 & 0.50 & 0.47 \\
\hline S14488 & $\mathrm{O}=\mathrm{c} 1 \mathrm{ncn} 2 \mathrm{c}(\mathrm{c} 1)[\mathrm{nH}] \mathrm{c}(=\mathrm{O}) \mathrm{cc} 2$ & 3.90 & 1.97 & 13.10 & 11.07 & 25.48 & 21.88 & 0.35 & 0.36 \\
\hline S14493 & $\mathrm{O}=\mathrm{c} 1 \mathrm{ccn} 2 \mathrm{c}(\mathrm{n} 1) \cos 2=\mathrm{O}$ & 3.30 & 3.57 & 10.91 & 10.59 & 22.21 & 19.31 & 0.35 & 0.36 \\
\hline S1450 & $\mathrm{O}=\mathrm{c} 1 \mathrm{ocnc} 2 \mathrm{c} 1 \mathrm{cno} 2$ & 3.45 & 3.27 & 8.76 & 7.61 & 12.32 & 12.20 & 0.52 & 0.53 \\
\hline S14507 & $\mathrm{O}=\mathrm{c} 1 \mathrm{ccn} 2 \mathrm{c}(\mathrm{n} 1) \operatorname{sc}(=\mathrm{O}) \mathrm{cc} 2$ & 3.53 & 2.88 & 14.76 & 12.89 & 26.14 & 24.39 & 0.56 & 0.53 \\
\hline S14511 & $\mathrm{O}=\mathrm{c} 1 \mathrm{ccn} 2 \mathrm{c}(\mathrm{n} 1) \mathrm{ncnc} 2$ & 3.46 & 3.03 & 14.75 & 12.22 & 28.26 & 22.99 & 0.55 & 0.44 \\
\hline S14519 & $\mathrm{O}=\mathrm{c} 1 \mathrm{ccn} 2 \mathrm{c}(\mathrm{n} 1) \mathrm{c}(=\mathrm{O})[\mathrm{nH}] \mathrm{nc} 2$ & 3.44 & 3.80 & 14.16 & 12.50 & 27.01 & 24.83 & 0.52 & 0.45 \\
\hline S14521 & $\mathrm{O}=\mathrm{c} 1 \mathrm{ccn} 2 \mathrm{c}(\mathrm{n} 1) \mathrm{cn}[\mathrm{nH}] \mathrm{c} 2=\mathrm{O}$ & 3.60 & 3.80 & 11.32 & 11.29 & 22.33 & 19.23 & 0.48 & 0.50 \\
\hline S14529 & $\mathrm{O}=\mathrm{c} 1[\mathrm{nH}] \mathrm{cc} 2 \mathrm{c}(\mathrm{n} 1)[\mathrm{nH}] \mathrm{cnc} 2=\mathrm{O}$ & 3.07 & 3.05 & 10.05 & 12.56 & 18.14 & 22.95 & 0.43 & 0.43 \\
\hline S1454 & $\mathrm{O}=\mathrm{c} 1 \mathrm{scnc} 2 \mathrm{c} 1 \mathrm{cno} 2$ & 2.57 & 2.58 & 7.43 & 6.88 & 11.66 & 11.52 & 0.75 & 0.84 \\
\hline
\end{tabular}




\begin{tabular}{|c|c|c|c|c|c|c|c|c|c|}
\hline S14565 & $\mathrm{O}=\mathrm{c} 1 \mathrm{ccn} 2 \mathrm{c}(\mathrm{n} 1)[\mathrm{nH}] \mathrm{c}(=\mathrm{O})[\mathrm{nH}] \mathrm{c} 2=\mathrm{O}$ & 5.04 & 4.57 & 14.00 & 11.52 & 23.88 & 19.44 & 0.39 & 0.34 \\
\hline S14585 & $\mathrm{O}=\mathrm{c} 1 \mathrm{ccn} 2 \mathrm{c}(\mathrm{n} 1)[\mathrm{nH}] \mathrm{ccc} 2=\mathrm{O}$ & 1.89 & 1.97 & 12.28 & 10.62 & 25.34 & 21.69 & 0.53 & 0.51 \\
\hline S14595 & $\mathrm{O}=\mathrm{c} 1 \mathrm{nc}[\mathrm{nH}] \mathrm{c} 2 \mathrm{c} 1 \mathrm{nccn} 2$ & 1.36 & 2.28 & 10.50 & 12.41 & 24.55 & 24.27 & 0.53 & 0.53 \\
\hline S14598 & $\mathrm{O}=\mathrm{c} 1 \mathrm{nc}[\mathrm{nH}] \mathrm{c} 2 \mathrm{c} 1 \mathrm{nncn} 2$ & 3.49 & 4.11 & 14.24 & 14.97 & 26.17 & 26.99 & 0.44 & 0.44 \\
\hline S14599 & $\mathrm{O}=\mathrm{c} 1 \mathrm{nc}[\mathrm{nH}] \mathrm{c} 2 \mathrm{c} 1 \mathrm{ncnn} 2$ & 3.55 & 4.11 & 12.91 & 13.10 & 23.29 & 22.93 & 0.40 & 0.42 \\
\hline S14604 & $\mathrm{O}=\mathrm{c} 1 \mathrm{nc}[\mathrm{nH}] \mathrm{c} 2 \mathrm{c} 1 \mathrm{c}(=\mathrm{O})[\mathrm{nH}] \mathrm{cn} 2$ & 1.15 & 3.05 & 11.75 & 14.55 & 28.68 & 28.75 & 0.45 & 0.38 \\
\hline S14605 & $\mathrm{O}=\mathrm{c} 1 \mathrm{nc}[\mathrm{nH}] \mathrm{c} 2 \mathrm{c} 1 \mathrm{nc}[\mathrm{nH}] \mathrm{c} 2=\mathrm{O}$ & 2.30 & 3.05 & 11.46 & 16.15 & 25.78 & 27.63 & 0.50 & 0.54 \\
\hline S14611 & $\mathrm{O}=\mathrm{c} 1 \mathrm{cnc} 2 \mathrm{c}(\mathrm{o} 1)[\mathrm{nH}] \mathrm{cnc} 2=\mathrm{O}$ & 4.15 & 4.65 & 15.34 & 14.98 & 27.40 & 27.07 & 0.59 & 0.63 \\
\hline S1462 & $\mathrm{O}=\mathrm{c} 1[\mathrm{nH}] \mathrm{nnc} 2 \mathrm{c} 1 \mathrm{cno} 2$ & 3.70 & 3.50 & 8.38 & 9.29 & 10.66 & 13.23 & 0.49 & 0.51 \\
\hline S1463 & $\mathrm{O}=\mathrm{c} 1[\mathrm{nH}] \mathrm{nnc} 2 \mathrm{c} 1 \mathrm{onc} 2$ & 3.52 & 3.50 & 7.92 & 8.81 & 12.36 & 13.05 & 0.41 & 0.41 \\
\hline S14647 & $\mathrm{O}=\mathrm{c} 1 \mathrm{ccn} 2 \mathrm{c}(\mathrm{n} 1) \mathrm{nn}[\mathrm{nH}] \mathrm{c} 2=\mathrm{O}$ & 4.98 & 5.63 & 14.33 & 15.31 & 25.28 & 24.07 & 0.62 & 0.60 \\
\hline S14675 & $\mathrm{O}=\operatorname{c} 1 \operatorname{ccn} 2 \mathrm{c}(\mathrm{n} 1) \operatorname{occc} 2=\mathrm{O}$ & 3.22 & 3.57 & 10.87 & 10.70 & 23.06 & 19.29 & 0.44 & 0.42 \\
\hline S1468 & $\mathrm{O}=\mathrm{c} 1 \mathrm{sn}[\mathrm{n}] \mathrm{c} 2 \mathrm{c} 1 \mathrm{cno} 2$ & 4.45 & 4.41 & 9.41 & 8.70 & 10.53 & 11.61 & 0.44 & 0.45 \\
\hline S14689 & $\mathrm{O}=\mathrm{c} 1 \operatorname{ccn} 2 \mathrm{c}(\mathrm{n} 1) \sec 2=\mathrm{O}$ & 2.53 & 2.88 & 9.98 & 9.88 & 22.10 & 18.55 & 0.60 & 0.58 \\
\hline S14693 & $\mathrm{O}=\mathrm{c} 1 \mathrm{ccn} 2 \mathrm{c}(\mathrm{n} 1)[\mathrm{nH}] \mathrm{ncc} 2=\mathrm{O}$ & 3.68 & 3.80 & 11.47 & 10.98 & 22.39 & 19.33 & 0.52 & 0.52 \\
\hline S14705 & $\mathrm{O}=\mathrm{c} 1 \mathrm{ccn} 2 \mathrm{c}(\mathrm{n} 1) \operatorname{snc} 2=\mathrm{O}$ & 4.35 & 4.71 & 12.64 & 11.69 & 22.74 & 18.08 & 0.42 & 0.40 \\
\hline S14717 & $\mathrm{O}=\mathrm{c} 1 \mathrm{ccn} 2 \mathrm{c}(\mathrm{n} 1)[\mathrm{nH}] \mathrm{nnc} 2=\mathrm{O}$ & 5.49 & 5.63 & 14.09 & 14.72 & 21.99 & 24.28 & 0.32 & 0.34 \\
\hline S1472 & $\mathrm{O}=\mathrm{c} 1 \mathrm{ccoc} 2 \mathrm{c} 1 \mathrm{onc} 2$ & 1.53 & 1.44 & 8.01 & 7.00 & 17.22 & 14.59 & 0.62 & 0.64 \\
\hline S1479 & $\mathrm{O}=\mathrm{c} 1 \mathrm{cn}[\mathrm{nH}] \mathrm{c} 2 \mathrm{c} 1 \mathrm{cno} 2$ & 1.90 & 1.67 & 8.93 & 7.91 & 16.22 & 15.59 & 0.51 & 0.54 \\
\hline S14797 & $\mathrm{O}=\mathrm{c} 1 \mathrm{ccc} 2 \mathrm{n} 1 \mathrm{cnsc} 2$ & 0.13 & -0.10 & 6.84 & 6.88 & 14.79 & 16.08 & 0.40 & 0.39 \\
\hline S1487 & $\mathrm{O}=\mathrm{c} 1 \mathrm{nn}[\mathrm{nH}] \mathrm{c} 2 \mathrm{c} 1 \mathrm{cno} 2$ & 3.62 & 3.50 & 11.69 & 11.60 & 19.95 & 21.29 & 0.47 & 0.51 \\
\hline S15162 & $\mathrm{O}=\mathrm{c} 1 \mathrm{ncn} 2 \mathrm{c}(\mathrm{n} 1) \sec 2$ & 2.31 & 1.73 & 12.74 & 11.17 & 28.33 & 23.11 & 0.37 & 0.40 \\
\hline S15188 & $\mathrm{O}=\mathrm{c} 1 \mathrm{ncn} 2 \mathrm{c}(\mathrm{n} 1) \mathrm{cncc} 2$ & 3.98 & 3.03 & 14.13 & 12.42 & 27.86 & 23.88 & 0.47 & 0.48 \\
\hline S15191 & $\mathrm{O}=\mathrm{c} 1 \mathrm{ncn} 2 \mathrm{c}(\mathrm{n} 1) \mathrm{nccc} 2$ & 3.51 & 3.03 & 15.49 & 13.96 & 31.75 & 27.87 & 0.41 & 0.38 \\
\hline S15195 & $\mathrm{O}=\mathrm{c} 1[\mathrm{nH}] \mathrm{ccn} 2 \mathrm{c} 1 \mathrm{nc}(=\mathrm{O}) \mathrm{nc} 2$ & 3.53 & 3.80 & 14.28 & 16.04 & 29.81 & 32.68 & 0.60 & 0.50 \\
\hline S15198 & $\mathrm{O}=\mathrm{c} 1 \mathrm{ncn} 2 \mathrm{c}(\mathrm{n} 1)[\mathrm{nH}] \mathrm{c}(=\mathrm{O}) \mathrm{cc} 2$ & 5.11 & 3.80 & 15.89 & 12.63 & 28.32 & 23.94 & 0.48 & 0.47 \\
\hline $\mathbf{S 1 5 2 0 7}$ & $\mathrm{O}=\mathrm{c} 1 \mathrm{ncn} 2 \mathrm{c}(\mathrm{n} 1) \mathrm{ncnc} 2$ & 5.38 & 4.86 & 17.47 & 14.01 & 30.44 & 24.47 & 0.43 & 0.41 \\
\hline S15271 & $\mathrm{O}=\mathrm{c} 1 \mathrm{n} \operatorname{cn} 2 \mathrm{c}(\mathrm{n} 1) \sec 2=\mathrm{O}$ & 4.54 & 4.71 & 12.66 & 12.63 & 25.23 & 22.55 & 0.62 & 0.61 \\
\hline S15307 & $\mathrm{O}=\mathrm{c} 1 \mathrm{ccc} 2 \mathrm{n} 1 \mathrm{cnsn} 2$ & 1.61 & 1.73 & 6.09 & 7.65 & 10.59 & 13.59 & 0.61 & 0.61 \\
\hline S15384 & $\mathrm{c} 1 \mathrm{cnc} 2 \mathrm{c}(\mathrm{n} 1) \mathrm{c}[\mathrm{nH}] \mathrm{c} 2$ & -3.72 & -2.53 & 6.54 & 4.68 & 14.50 & 13.27 & 0.45 & 0.55 \\
\hline S15386 & $\operatorname{c} 1 \mathrm{cnc} 2 \mathrm{c}(\mathrm{n} 1) \csc 2$ & -2.18 & -1.62 & 1.60 & 2.93 & 6.39 & 9.35 & 0.83 & 0.82 \\
\hline
\end{tabular}




\begin{tabular}{|c|c|c|c|c|c|c|c|c|c|}
\hline S15387 & $\mathrm{c} 1 \mathrm{cnc} 2 \mathrm{c}(\mathrm{n} 1) \mathrm{n}[\mathrm{nH}] \mathrm{c} 2$ & -1.83 & -0.70 & 6.58 & 5.91 & 15.64 & 13.53 & 0.40 & 0.39 \\
\hline S15390 & $\mathrm{c} 1 \mathrm{cnc} 2 \mathrm{c}(\mathrm{n} 1) \mathrm{n}[\mathrm{nH}] \mathrm{n} 2$ & 0.11 & 1.13 & 5.32 & 6.12 & 10.01 & 11.54 & 0.45 & 0.44 \\
\hline S15391 & c1cnc2c(n1)non2 & 2.98 & 2.73 & 10.02 & 7.03 & 14.28 & 10.92 & 0.72 & 0.73 \\
\hline S15392 & $\mathrm{c} 1 \mathrm{cnc} 2 \mathrm{c}(\mathrm{n} 1) \mathrm{nsn} 2$ & 1.54 & 2.04 & 5.87 & 6.15 & 9.37 & 9.96 & 0.52 & 0.47 \\
\hline S15402 & c1cnc $2 \mathrm{c}(\mathrm{n} 1) \mathrm{nccn} 2$ & 0.35 & 1.51 & 4.07 & 5.94 & 9.17 & 11.03 & 0.44 & 0.48 \\
\hline S15404 & $\mathrm{O}=\mathrm{c} 1[\mathrm{nH}] \mathrm{cnc} 2 \mathrm{c} 1 \mathrm{nccn} 2$ & 0.74 & 2.28 & 8.39 & 8.38 & 16.70 & 14.36 & 0.47 & 0.49 \\
\hline S15406 & $\mathrm{O}=\mathrm{c} 1 \mathrm{ocnc} 2 \mathrm{c} 1 \mathrm{nccn} 2$ & 2.49 & 3.88 & 6.36 & 8.25 & 13.37 & 12.95 & 0.39 & 0.38 \\
\hline S15408 & $\mathrm{O}=\mathrm{c} 1 \mathrm{scnc} 2 \mathrm{c} 1 \mathrm{nccn} 2$ & 1.39 & 3.19 & 5.26 & 7.45 & 12.21 & 12.09 & 0.54 & 0.53 \\
\hline S15441 & $\mathrm{c} 1 \mathrm{nnc} 2 \mathrm{c}(\mathrm{n} 1) \mathrm{c}[\mathrm{nH}] \mathrm{c} 2$ & -1.58 & -0.70 & 10.16 & 7.33 & 20.24 & 15.80 & 0.62 & 0.60 \\
\hline S15443 & $\mathrm{c} 1 \mathrm{nnc} 2 \mathrm{c}(\mathrm{n} 1) \csc 2$ & -0.13 & 0.21 & 4.10 & 4.82 & 10.94 & 10.89 & 0.51 & 0.47 \\
\hline S15444 & $\mathrm{c} 1 \mathrm{nnc} 2 \mathrm{c}(\mathrm{n} 1) \mathrm{c}[\mathrm{nH}] \mathrm{n} 2$ & 0.30 & 1.13 & 10.31 & 8.72 & 19.31 & 16.41 & 0.57 & 0.56 \\
\hline S15445 & $\mathrm{c} 1 \mathrm{nnc} 2 \mathrm{c}(\mathrm{n} 1) \mathrm{n}[\mathrm{nH}] \mathrm{c} 2$ & 0.28 & 1.13 & 10.41 & 8.05 & 17.22 & 14.94 & 0.36 & 0.35 \\
\hline $\mathbf{S 1 5 4 5 0}$ & $\mathrm{c} 1 \mathrm{nnc} 2 \mathrm{c}(\mathrm{n} 1) \mathrm{n}[\mathrm{nH}] \mathrm{n} 2$ & 2.16 & 2.96 & 9.11 & 8.42 & 14.15 & 13.29 & 0.90 & 0.87 \\
\hline S15473 & $\mathrm{O}=\mathrm{c} 1[\mathrm{nH}] \mathrm{cnc} 2 \mathrm{c} 1 \mathrm{nncn} 2$ & 2.95 & 4.11 & 12.31 & 10.84 & 19.24 & 16.91 & 0.52 & 0.48 \\
\hline S15474 & $\mathrm{O}=\mathrm{c} 1[\mathrm{nH}] \mathrm{cnc} 2 \mathrm{c} 1 \mathrm{ncnn} 2$ & 3.07 & 4.11 & 11.95 & 11.08 & 18.00 & 16.74 & 0.49 & 0.50 \\
\hline S15481 & $\mathrm{O}=\mathrm{c} 1 \mathrm{scn} c 2 \mathrm{c} 1 \mathrm{nn} \mathrm{cn} 2$ & 3.56 & 5.02 & 8.92 & 9.49 & 14.56 & 14.09 & 0.44 & 0.42 \\
\hline S15482 & $\mathrm{O}=\mathrm{c} 1 \mathrm{scnc} 2 \mathrm{c} 1 \mathrm{ncnn} 2$ & 3.67 & 5.02 & 8.95 & 9.35 & 13.03 & 13.42 & 0.48 & 0.51 \\
\hline $\mathbf{S 1 5 5 2 0}$ & $\mathrm{O}=\mathrm{c} 1 \mathrm{ccc}(=\mathrm{O}) \mathrm{n} 2 \mathrm{n} 1 \mathrm{cncc} 2$ & 2.35 & 2.72 & 7.22 & 8.53 & 11.35 & 15.55 & 0.37 & 0.36 \\
\hline $\mathbf{S 1 5 6 0 2}$ & $\mathrm{O}=\mathrm{c} 1 \mathrm{ccnc} 2 \mathrm{n} 1 \mathrm{cc}[\mathrm{nH}] 2$ & -1.59 & -1.01 & 8.53 & 6.06 & 19.79 & 15.42 & 0.52 & 0.51 \\
\hline S15603 & $\mathrm{O}=\mathrm{c} 1[\mathrm{nH}] \mathrm{cnc} 2 \mathrm{c} 1 \mathrm{c}[\mathrm{nH}] \mathrm{c} 2$ & -3.07 & -1.76 & 6.41 & 6.46 & 16.53 & 15.61 & 0.62 & 0.63 \\
\hline S15605 & $\mathrm{O}=\mathrm{c} 1 \mathrm{ccnc} 2 \mathrm{n} 1 \mathrm{cco} 2$ & 0.31 & 0.59 & 6.65 & 6.14 & 14.33 & 12.77 & 0.51 & 0.50 \\
\hline S15606 & $\mathrm{O}=\mathrm{c} 1[\mathrm{nH}] \mathrm{cnc} 2 \mathrm{c} 1 \operatorname{coc} 2$ & -0.80 & -0.16 & 5.91 & 6.37 & 12.75 & 13.22 & 0.60 & 0.54 \\
\hline $\mathbf{S 1 5 6 0 9}$ & $\mathrm{O}=\mathrm{c} 1[\mathrm{nH}] \mathrm{cnc} 2 \mathrm{c} 1 \mathrm{csc} 2$ & -1.50 & -0.85 & 5.60 & 5.56 & 12.86 & 12.48 & 0.50 & 0.56 \\
\hline S15611 & $\mathrm{O}=\mathrm{c} 1 \mathrm{ccnc} 2 \mathrm{n} 1 \mathrm{cn}[\mathrm{nH}] 2$ & 0.62 & 0.82 & 6.33 & 7.45 & 14.14 & 15.61 & 0.39 & 0.44 \\
\hline S15612 & $\mathrm{O}=\mathrm{c} 1[\mathrm{nH}] \mathrm{cnc} 2 \mathrm{c} 1 \mathrm{n}[\mathrm{nH}] \mathrm{c} 2$ & -1.42 & 0.07 & 6.13 & 7.62 & 17.50 & 16.54 & 0.47 & 0.47 \\
\hline S15613 & $\mathrm{O}=\mathrm{c} 1[\mathrm{nH}] \mathrm{cnc} 2 \mathrm{c} 1 \mathrm{c}[\mathrm{nH}] \mathrm{n} 2$ & -1.06 & 0.07 & 6.19 & 8.19 & 14.59 & 16.08 & 0.57 & 0.56 \\
\hline S15616 & $\mathrm{O}=\mathrm{c} 1[\mathrm{nH}] \mathrm{cnc} 2 \mathrm{c} 1 \mathrm{noc} 2$ & 1.17 & 1.67 & 9.10 & 8.48 & 17.39 & 15.48 & 0.81 & 0.87 \\
\hline S15617 & $\mathrm{O}=\mathrm{c} 1[\mathrm{nH}] \mathrm{cnc} 2 \mathrm{c} 1 \mathrm{con} 2$ & 1.56 & 1.67 & 9.40 & 9.05 & 17.27 & 15.02 & 0.39 & 0.34 \\
\hline S15619 & $\mathrm{O}=\mathrm{c} 1 \mathrm{ccnc} 2 \mathrm{n} 1 \mathrm{cns} 2$ & 1.72 & 1.73 & 6.07 & 7.60 & 11.27 & 13.43 & 0.49 & 0.47 \\
\hline $\mathbf{S 1 5 6 2 2}$ & $\mathrm{O}=\mathrm{c} 1 \mathrm{ccnc} 2 \mathrm{n} 1[\mathrm{nH}] \mathrm{cn} 2$ & 0.63 & 0.82 & 10.85 & 10.19 & 18.34 & 18.05 & 0.52 & 0.58 \\
\hline S15627 & $\mathrm{O}=\mathrm{c} 1 \mathrm{ccnc} 2 \mathrm{n} 1 \mathrm{ncs} 2$ & 0.59 & 1.73 & 7.72 & 6.77 & 18.04 & 13.84 & 0.45 & 0.43 \\
\hline
\end{tabular}




\begin{tabular}{|c|c|c|c|c|c|c|c|c|c|}
\hline $\mathbf{S 1 5 6 3 0}$ & $\mathrm{O}=\mathrm{c} 1[\mathrm{nH}] \mathrm{cnc} 2 \mathrm{c} 1 \mathrm{n}[\mathrm{nH}] \mathrm{n} 2$ & 0.66 & 1.90 & 7.77 & 8.33 & 15.56 & 14.77 & 0.47 & 0.46 \\
\hline S15636 & $\mathrm{O}=\mathrm{c} 1[\mathrm{nH}] \mathrm{cnc} 2 \mathrm{c} 1 \mathrm{non} 2$ & 3.58 & 3.50 & 12.92 & 10.14 & 17.53 & 15.04 & 0.46 & 0.44 \\
\hline S15637 & $\mathrm{O}=\mathrm{c} 1[\mathrm{nH}] \mathrm{cnc} 2 \mathrm{c} 1 \mathrm{nsn} 2$ & 2.05 & 2.81 & 9.90 & 9.21 & 16.33 & 13.98 & 0.56 & 0.53 \\
\hline S15643 & $\mathrm{O}=\mathrm{c} 1 \mathrm{ccnc} 2 \mathrm{n} 1 \mathrm{ccc} 2$ & -0.94 & -0.63 & 5.41 & 5.13 & 14.05 & 12.95 & 0.53 & 0.48 \\
\hline S15644 & $\mathrm{O}=\mathrm{c} 1 \mathrm{ccnc} 2 \mathrm{n} 1 \mathrm{ccnc} 2$ & 1.10 & 1.20 & 4.93 & 7.24 & 10.83 & 14.04 & 0.50 & 0.52 \\
\hline S15645 & $\mathrm{O}=\mathrm{c} 1 \mathrm{ccnc} 2 \mathrm{n} 1 \mathrm{cnc} 2$ & 1.01 & 1.20 & 4.81 & 7.11 & 11.21 & 14.40 & 0.53 & 0.51 \\
\hline S15646 & $\mathrm{O}=\mathrm{c} 1 \mathrm{ccnc} 2 \mathrm{n} 1 \mathrm{nccc} 2$ & -0.09 & 1.20 & 8.01 & 6.78 & 19.70 & 14.82 & 0.43 & 0.41 \\
\hline S15647 & $\mathrm{O}=\mathrm{c} 1 \mathrm{ccnc} 2 \mathrm{n} 1 \mathrm{ccc} 2$ & 0.60 & 1.20 & 7.22 & 7.34 & 14.16 & 14.14 & 0.47 & 0.44 \\
\hline S15651 & $\mathrm{O}=\mathrm{c} 1 \mathrm{ccnc} 2 \mathrm{n} 1 \mathrm{cc}[\mathrm{nH}] \mathrm{c} 2=\mathrm{O}$ & 0.84 & 1.97 & 9.04 & 8.75 & 17.46 & 16.97 & 0.36 & 0.39 \\
\hline $\mathbf{S 1 5 6 5 2}$ & $\mathrm{O}=\mathrm{c} 1 \mathrm{ccnc} 2 \mathrm{n} 1 \mathrm{c}(=\mathrm{O})[\mathrm{nH}] \mathrm{cc} 2$ & 0.07 & 1.97 & 10.16 & 8.28 & 22.69 & 17.63 & 0.36 & 0.38 \\
\hline S15654 & $\mathrm{O}=\mathrm{c} 1 \mathrm{ccn} 2 \mathrm{c}([\mathrm{nH}] 1) \mathrm{nccc} 2=\mathrm{O}$ & 2.32 & 1.97 & 7.36 & 8.52 & 12.57 & 16.73 & 0.57 & 0.56 \\
\hline S15666 & $\mathrm{O}=\mathrm{c} 1 \mathrm{ccn} 2 \mathrm{c}(\mathrm{s} 1) \mathrm{nccc} 2=\mathrm{O}$ & 2.55 & 2.88 & 7.50 & 9.18 & 12.45 & 15.93 & 0.44 & 0.45 \\
\hline S15669 & $\mathrm{O}=\mathrm{c} 1 \mathrm{ccnc} 2 \mathrm{n} 1 \mathrm{cncn} 2$ & 2.63 & 3.03 & 8.16 & 8.72 & 12.04 & 14.30 & 0.59 & 0.63 \\
\hline S15673 & $\mathrm{O}=\mathrm{c} 1 \mathrm{ccnc} 2 \mathrm{n} 1 \mathrm{cn}[\mathrm{nH}] \mathrm{c} 2=\mathrm{O}$ & 2.87 & 3.80 & 8.55 & 8.93 & 15.25 & 14.83 & 0.50 & 0.48 \\
\hline S15675 & $\mathrm{O}=\mathrm{c} 1[\mathrm{nH}] \mathrm{nc} 2 \mathrm{c}(\mathrm{c} 1) \mathrm{c}(=\mathrm{O})[\mathrm{nH}] \mathrm{cn} 2$ & 2.40 & 3.05 & 10.99 & 9.17 & 19.36 & 15.48 & 0.59 & 0.58 \\
\hline S15676 & $\mathrm{O}=\mathrm{c} 1[\mathrm{nH}] \mathrm{nc} 2 \mathrm{c}(\mathrm{c} 1) \mathrm{nc}[\mathrm{nH}] \mathrm{c} 2=\mathrm{O}$ & 2.14 & 3.05 & 11.00 & 9.06 & 19.34 & 15.55 & 0.68 & 0.67 \\
\hline S15679 & $\mathrm{O}=\mathrm{c} 1[\mathrm{nH}] \mathrm{cc} 2 \mathrm{c}(\mathrm{n} 1) \mathrm{nc}[\mathrm{nH}] \mathrm{c} 2=\mathrm{O}$ & 2.45 & 3.05 & 10.56 & 13.25 & 22.93 & 22.87 & 0.68 & 0.69 \\
\hline S15702 & $\mathrm{O}=\mathrm{c} 1[\mathrm{nH}] \mathrm{c} 2 \mathrm{nccc}(=\mathrm{O}) \mathrm{n} 2 \mathrm{c}(=\mathrm{O})[\mathrm{nH}] 1$ & 3.56 & 4.57 & 10.96 & 10.22 & 19.29 & 18.07 & 0.55 & 0.71 \\
\hline S15712 & $\mathrm{O}=\mathrm{c} 1 \mathrm{ccnc} 2 \mathrm{n} 1 \mathrm{c}(=\mathrm{O}) \mathrm{cc}[\mathrm{nH}] 2$ & 0.17 & 1.97 & 11.24 & 10.53 & 25.07 & 22.80 & 0.40 & 0.35 \\
\hline S15718 & $\mathrm{O}=\mathrm{c} 1 \mathrm{ccnc} 2 \mathrm{n} 1 \mathrm{ncnn} 2$ & 3.45 & 4.86 & 9.45 & 10.50 & 15.47 & 15.81 & 0.45 & 0.49 \\
\hline S15719 & $\mathrm{O}=\mathrm{c} 1[\mathrm{nH}] \mathrm{cnc} 2 \mathrm{c} 1 \mathrm{c}(=\mathrm{O})[\mathrm{nH}] \mathrm{cn} 2$ & 0.64 & 3.05 & 8.03 & 10.38 & 19.72 & 17.17 & 0.53 & 0.49 \\
\hline S15720 & $\mathrm{O}=\mathrm{c} 1[\mathrm{nH}] \mathrm{cnc} 2 \mathrm{c} 1 \mathrm{nc}[\mathrm{nH}] \mathrm{c} 2=\mathrm{O}$ & 1.02 & 3.05 & 7.81 & 10.18 & 16.23 & 17.12 & 0.41 & 0.39 \\
\hline S15724 & $\mathrm{O}=\mathrm{c} 1 \mathrm{cnc} 2 \mathrm{c}(\mathrm{o} 1) \mathrm{nc}[\mathrm{nH}] \mathrm{c} 2=\mathrm{O}$ & 3.43 & 4.65 & 12.70 & 11.23 & 18.93 & 17.55 & 0.50 & 0.49 \\
\hline S15850 & $\mathrm{O}=\mathrm{c} 1 \mathrm{cnc} 2 \mathrm{c}(\mathrm{o} 1) \operatorname{coc} 2$ & 1.28 & 1.44 & 3.93 & 6.01 & 10.25 & 12.42 & 0.53 & 0.45 \\
\hline S15851 & $\mathrm{O}=\mathrm{c} 1 \mathrm{cnc} 2 \mathrm{c}(\mathrm{o} 1) \csc 2$ & 0.67 & 0.75 & 3.33 & 5.32 & 10.21 & 11.99 & 0.45 & 0.51 \\
\hline S15861 & $\mathrm{O}=\mathrm{c} 1[\mathrm{nH}] \mathrm{cc} 2 \mathrm{c}(\mathrm{c} 1) \mathrm{oc}(=\mathrm{O}) \mathrm{cn} 2$ & 2.98 & 2.82 & 10.18 & 8.88 & 17.22 & 15.31 & 0.68 & 0.66 \\
\hline S15862 & $\mathrm{O}=\mathrm{c} 1[\mathrm{nH}] \mathrm{cc} 2 \mathrm{c}(\mathrm{c} 1) \mathrm{ncc}(=\mathrm{O}) \mathrm{o} 2$ & 3.15 & 2.82 & 9.13 & 9.19 & 16.83 & 16.34 & 0.52 & 0.51 \\
\hline S15869 & $\mathrm{O}=\mathrm{c} 1[\mathrm{nH}] \mathrm{cc} 2 \mathrm{c}(\mathrm{n} 1) \mathrm{ncc}(=\mathrm{O}) \mathrm{o} 2$ & 4.61 & 4.65 & 12.72 & 13.07 & 22.52 & 22.36 & 0.52 & 0.51 \\
\hline S15870 & $\mathrm{O}=\mathrm{c} 1[\mathrm{nH}] \mathrm{cc} 2 \mathrm{c}(\mathrm{n} 1) \mathrm{oc}(=\mathrm{O}) \mathrm{cn} 2$ & 4.37 & 4.65 & 13.41 & 12.39 & 22.23 & 20.07 & 0.62 & 0.61 \\
\hline S1596 & {$[\mathrm{nH}] 1 \mathrm{cc} 2 \mathrm{c}(\mathrm{c} 1) \mathrm{cns} 2$} & -4.17 & -3.83 & 7.31 & 5.07 & 18.97 & 15.21 & 0.41 & 0.41 \\
\hline S1597 & o1cc2c(c1)cns2 & -2.10 & -2.23 & 3.33 & 2.96 & 10.49 & 10.48 & 0.37 & 0.34 \\
\hline
\end{tabular}




\begin{tabular}{|c|c|c|c|c|c|c|c|c|c|}
\hline S1598 & $\mathrm{s} 1 \mathrm{cc} 2 \mathrm{c}(\mathrm{c} 1) \mathrm{cns} 2$ & -2.50 & -2.92 & 3.01 & 2.28 & 10.62 & 10.02 & 0.33 & 0.34 \\
\hline S1599 & $\mathrm{n} 1 \mathrm{cc} 2 \mathrm{c}(\mathrm{s} 1) \mathrm{n}[\mathrm{nH}] \mathrm{c} 2$ & -2.40 & -2.00 & 7.44 & 6.00 & 16.18 & 14.34 & 0.58 & 0.47 \\
\hline S16 & c1cnsn1 & -0.44 & -0.66 & 3.50 & 3.30 & 6.81 & 9.51 & 0.47 & 0.46 \\
\hline S1600 & $\mathrm{n} 1 \mathrm{cc} 2 \mathrm{c}(\mathrm{s} 1) \mathrm{c}[\mathrm{nH}] \mathrm{n} 2$ & -2.51 & -2.00 & 7.15 & 5.50 & 15.59 & 14.13 & 0.58 & 0.58 \\
\hline S1603 & $\mathrm{n} 1 \mathrm{cc} 2 \mathrm{c}(\mathrm{s} 1) \mathrm{snc} 2$ & -0.42 & -1.09 & 3.61 & 3.57 & 7.40 & 9.32 & 0.52 & 0.57 \\
\hline S1604 & $\mathrm{n} 1 \mathrm{cc} 2 \mathrm{c}(\mathrm{s} 1) \mathrm{cns} 2$ & -0.43 & -1.09 & 2.85 & 3.42 & 6.58 & 9.30 & 0.35 & 0.38 \\
\hline S1605 & $\mathrm{n} 1 \mathrm{cc} 2 \mathrm{c}(\mathrm{s} 1) \mathrm{nsc} 2$ & -0.78 & -1.09 & 6.31 & 4.10 & 11.85 & 10.33 & 0.32 & 0.33 \\
\hline S1607 & $\mathrm{c} 1 \mathrm{nc} 2 \mathrm{c}([\mathrm{nH}] 1) \mathrm{snc} 2$ & -2.05 & -2.00 & 9.14 & 6.51 & 17.39 & 16.15 & 0.56 & 0.57 \\
\hline S1608 & $\mathrm{c} 1 \mathrm{nc} 2 \mathrm{c}([\mathrm{nH}] 1) \mathrm{cns} 2$ & -1.91 & -2.00 & 9.57 & 7.57 & 17.95 & 16.53 & 0.69 & 0.60 \\
\hline S1609 & c1nc2c(o1)snc2 & -0.30 & -0.40 & 4.55 & 3.99 & 9.71 & 10.29 & 0.64 & 0.57 \\
\hline S161 & $\mathrm{O}=\mathrm{c} 1 \mathrm{occoc} 1=\mathrm{O}$ & 3.25 & 3.55 & 8.74 & 9.72 & 15.44 & 21.61 & 0.69 & 0.65 \\
\hline S1610 & c1oc2c(n1)snc2 & -0.23 & -0.40 & 4.87 & 4.29 & 10.13 & 10.46 & 0.48 & 0.44 \\
\hline S1611 & $\mathrm{c} 1 \mathrm{nc} 2 \mathrm{c}(\mathrm{s} 1) \mathrm{snc} 2$ & -0.99 & -1.09 & 4.04 & 3.34 & 9.74 & 9.97 & 0.43 & 0.45 \\
\hline S1612 & $\mathrm{c} 1 \mathrm{sc} 2 \mathrm{c}(\mathrm{n} 1) \mathrm{snc} 2$ & -0.90 & -1.09 & 4.18 & 3.69 & 9.92 & 10.17 & 0.59 & 0.63 \\
\hline S1613 & $\mathrm{n} 1 \mathrm{cc} 2 \mathrm{c}(\mathrm{s} 1)[\mathrm{nH}] \mathrm{nn} 2$ & 0.01 & -0.17 & 8.54 & 7.31 & 15.17 & 15.60 & 0.41 & 0.40 \\
\hline S1614 & $\mathrm{n} 1 \mathrm{sc} 2 \mathrm{c}(\mathrm{c} 1)[\mathrm{nH}] \mathrm{nn} 2$ & 0.14 & -0.17 & 9.36 & 8.37 & 16.41 & 15.97 & 0.48 & 0.49 \\
\hline S1615 & {$[\mathrm{nH}] 1 \mathrm{nc} 2 \mathrm{c}(\mathrm{n} 1) \mathrm{cns} 2$} & -0.73 & -0.17 & 6.06 & 5.41 & 11.31 & 11.01 & 0.67 & 0.68 \\
\hline S1628 & $\operatorname{c} 1 \mathrm{ccc} 2 \mathrm{c}(\mathrm{c} 1) \operatorname{snc} 2$ & -2.73 & -3.45 & 1.83 & 2.52 & 9.45 & 11.03 & 0.48 & 0.45 \\
\hline S1629 & $\mathrm{c} 1 \mathrm{ncc} 2 \mathrm{c}(\mathrm{c} 1) \mathrm{snc} 2$ & -0.80 & -1.62 & 3.02 & 3.45 & 7.46 & 10.44 & 0.49 & 0.46 \\
\hline S1630 & $\mathrm{c} 1 \mathrm{ncc} 2 \mathrm{c}(\mathrm{c} 1) \mathrm{cns} 2$ & -0.82 & -1.62 & 3.87 & 3.65 & 8.48 & 10.52 & 0.63 & 0.60 \\
\hline S1631 & $\mathrm{c} 1 \mathrm{cnc} 2 \mathrm{c}(\mathrm{c} 1) \mathrm{cns} 2$ & -1.23 & -1.62 & 4.99 & 4.45 & 11.36 & 11.81 & 0.37 & 0.34 \\
\hline S1632 & $\mathrm{c} 1 \mathrm{cnc} 2 \mathrm{c}(\mathrm{c} 1) \mathrm{snc} 2$ & -1.33 & -1.62 & 4.39 & 3.91 & 10.71 & 11.58 & 0.50 & 0.57 \\
\hline S16332 & $\mathrm{O}=\mathrm{c} 1 \mathrm{ncnc} 2 \mathrm{n} 1[\mathrm{nH}] \mathrm{cc} 2$ & 0.78 & 0.82 & 11.46 & 8.96 & 22.47 & 17.55 & 0.52 & 0.47 \\
\hline S16338 & $\mathrm{O}=\mathrm{c} 1 \mathrm{ncnc} 2 \mathrm{n} 1[\mathrm{nH}] \mathrm{nc} 2$ & 2.74 & 2.65 & 9.13 & 9.81 & 17.13 & 16.57 & 0.51 & 0.59 \\
\hline S16344 & $\mathrm{O}=\mathrm{c} 1 \mathrm{ncnc} 2 \mathrm{n} 1[\mathrm{nH}] \mathrm{cn} 2$ & 2.66 & 2.65 & 14.17 & 11.93 & 22.39 & 19.52 & 0.52 & 0.51 \\
\hline S1635 & $\mathrm{n} 1 \mathrm{ncc} 2 \mathrm{c}(\mathrm{c} 1) \mathrm{snc} 2$ & 1.32 & 0.21 & 5.75 & 5.60 & 13.27 & 12.17 & 0.45 & 0.48 \\
\hline S1636 & $\mathrm{O}=\mathrm{c} 1[\mathrm{nH}] \mathrm{ccc} 2 \mathrm{c} 1 \mathrm{cns} 2$ & -0.64 & -0.85 & 8.24 & 6.55 & 16.55 & 15.48 & 0.50 & 0.47 \\
\hline S16518 & s1cc2c(c1)nnnn2 & 2.04 & 2.04 & 7.78 & 7.74 & 16.08 & 14.67 & 0.64 & 0.60 \\
\hline S1654 & $\mathrm{c} 1 \mathrm{ncc} 2 \mathrm{c}(\mathrm{n} 1) \mathrm{cns} 2$ & 0.61 & 0.21 & 4.36 & 4.45 & 7.23 & 9.78 & 0.66 & 0.68 \\
\hline S1655 & $\mathrm{c} 1 \mathrm{ncc} 2 \mathrm{c}(\mathrm{n} 1) \mathrm{snc} 2$ & 0.72 & 0.21 & 5.57 & 4.80 & 8.60 & 9.92 & 0.62 & 0.60 \\
\hline S1658 & $\mathrm{O}=\mathrm{c} 1[\mathrm{nH}] \mathrm{ncc} 2 \mathrm{c} 1 \mathrm{cns} 2$ & 1.21 & 0.98 & 5.84 & 5.89 & 12.47 & 11.38 & 0.44 & 0.42 \\
\hline
\end{tabular}




\begin{tabular}{|c|c|c|c|c|c|c|c|c|c|}
\hline S1659 & $\mathrm{O}=\mathrm{c} 1[\mathrm{nH}] \mathrm{ncc} 2 \mathrm{c} 1 \mathrm{snc} 2$ & 1.28 & 0.98 & 6.30 & 6.07 & 13.29 & 11.55 & 0.47 & 0.49 \\
\hline S16626 & $\mathrm{O}=\mathrm{c} 1[\mathrm{nH}] \mathrm{nnc} 2 \mathrm{c} 1 \mathrm{c}[\mathrm{nH}] \mathrm{c} 2$ & -1.17 & 0.07 & 10.10 & 7.91 & 18.81 & 15.85 & 0.49 & 0.54 \\
\hline S16627 & $\mathrm{O}=\mathrm{c} 1[\mathrm{nH}] \mathrm{nnc} 2 \mathrm{c} 1 \operatorname{coc} 2$ & 1.01 & 1.67 & 4.06 & 7.01 & 9.53 & 12.32 & 0.43 & 0.40 \\
\hline S16628 & $\mathrm{O}=\mathrm{c} 1[\mathrm{nH}] \mathrm{nnc} 2 \mathrm{c} 1 \mathrm{csc} 2$ & 0.35 & 0.98 & 3.89 & 6.25 & 9.83 & 11.72 & 0.43 & 0.46 \\
\hline S16629 & $\mathrm{O}=\mathrm{c} 1[\mathrm{nH}] \mathrm{nnc} 2 \mathrm{c} 1 \mathrm{c}[\mathrm{nH}] \mathrm{n} 2$ & 0.80 & 1.90 & 9.99 & 9.80 & 17.11 & 16.67 & 0.42 & 0.41 \\
\hline S16630 & $\mathrm{O}=\mathrm{c} 1[\mathrm{nH}] \mathrm{nnc} 2 \mathrm{c} 1 \mathrm{n}[\mathrm{nH}] \mathrm{c} 2$ & 0.46 & 1.90 & 10.02 & 8.56 & 19.28 & 15.66 & 0.67 & 0.68 \\
\hline S16632 & $\mathrm{O}=\mathrm{c} 1[\mathrm{nH}] \mathrm{nnc} 2 \mathrm{c} 1 \mathrm{noc} 2$ & 2.93 & 3.50 & 7.27 & 8.61 & 13.34 & 13.47 & 0.44 & 0.41 \\
\hline S16639 & $\mathrm{O}=\mathrm{c} 1[\mathrm{nH}] \mathrm{cc} 2 \mathrm{c}(\mathrm{c} 1) \mathrm{c}(=\mathrm{O})[\mathrm{nH}] \mathrm{nn} 2$ & 2.59 & 3.05 & 10.26 & 9.21 & 16.91 & 15.73 & 0.45 & 0.47 \\
\hline S1687 & $\mathrm{O}=\mathrm{c} 1[\mathrm{nH}] \mathrm{c}(=\mathrm{O}) \mathrm{c} 2 \mathrm{c}([\mathrm{nH}] 1) \mathrm{cns} 2$ & 2.71 & 1.75 & 9.53 & 8.48 & 14.79 & 14.31 & 0.38 & 0.40 \\
\hline $\mathbf{S 1 6 9 2 0}$ & $\mathrm{O}=\mathrm{c} 1 \mathrm{nnc} 2 \mathrm{c}(\mathrm{o} 1) \mathrm{csc} 2$ & 2.50 & 2.58 & 6.91 & 8.69 & 15.70 & 17.74 & 0.53 & 0.51 \\
\hline S1701 & $\mathrm{c} 1 \mathrm{cnc} 2 \mathrm{c}(\mathrm{n} 1) \mathrm{snc} 2$ & 0.15 & 0.21 & 4.75 & 4.83 & 9.35 & 10.12 & 0.53 & 0.53 \\
\hline S1702 & $\mathrm{c} 1 \mathrm{nnnc} 2 \mathrm{c}(\mathrm{n} 1) \mathrm{cns} 2$ & 2.21 & 2.04 & 5.68 & 6.39 & 9.35 & 10.56 & 0.40 & 0.41 \\
\hline S1703 & c1nnc2c(n1)snc2 & 2.22 & 2.04 & 5.52 & 6.19 & 8.97 & 10.48 & 0.52 & 0.53 \\
\hline S1704 & $\mathrm{O}=\mathrm{c} 1[\mathrm{nH}] \mathrm{cnc} 2 \mathrm{c} 1 \mathrm{snc} 2$ & 0.79 & 0.98 & 9.31 & 7.86 & 15.19 & 14.16 & 0.42 & 0.43 \\
\hline S1705 & $\mathrm{O}=\mathrm{c} 1[\mathrm{nH}] \mathrm{cnc} 2 \mathrm{c} 1 \mathrm{cns} 2$ & 0.83 & 0.98 & 9.93 & 8.03 & 15.54 & 14.13 & 0.47 & 0.48 \\
\hline S1707 & $\mathrm{O}=\mathrm{c} 1 \mathrm{cnc} 2 \mathrm{c}(\mathrm{o} 1) \mathrm{cns} 2$ & 2.94 & 2.58 & 7.51 & 6.77 & 11.83 & 11.19 & 0.51 & 0.46 \\
\hline S1709 & $\mathrm{O}=\mathrm{c} 1 \mathrm{ocnc} 2 \mathrm{c} 1 \mathrm{cns} 2$ & 2.43 & 2.58 & 7.29 & 6.79 & 12.09 & 11.43 & 0.35 & 0.37 \\
\hline S1713 & $\mathrm{O}=\mathrm{c} 1 \mathrm{scnc} 2 \mathrm{c} 1 \mathrm{cns} 2$ & 1.57 & 1.89 & 6.43 & 6.06 & 10.83 & 10.73 & 0.37 & 0.38 \\
\hline S1721 & $\mathrm{O}=\mathrm{c} 1[\mathrm{nH}] \mathrm{nnc} 2 \mathrm{c} 1 \mathrm{cns} 2$ & 2.64 & 2.81 & 6.62 & 8.39 & 10.03 & 12.28 & 0.42 & 0.46 \\
\hline S1722 & $\mathrm{O}=\mathrm{c} 1[\mathrm{nH}] \mathrm{nnc} 2 \mathrm{c} 1 \mathrm{snc} 2$ & 2.64 & 2.81 & 6.42 & 8.02 & 10.16 & 12.23 & 0.60 & 0.58 \\
\hline S1727 & $\mathrm{O}=\mathrm{c} 1 \mathrm{sn}[\mathrm{n}] \mathrm{c} 2 \mathrm{c} 1 \mathrm{cns} 2$ & 3.42 & 3.72 & 7.61 & 7.86 & 10.31 & 10.77 & 0.84 & 0.84 \\
\hline S17553 & $\mathrm{O}=\mathrm{c} 1 \mathrm{c} \operatorname{coc} 2 \mathrm{c} 1 \mathrm{c}(=\mathrm{O}) \mathrm{cco} 2$ & 0.69 & 2.59 & 7.54 & 8.30 & 21.72 & 19.91 & 0.42 & 0.40 \\
\hline S17554 & $\mathrm{O}=\mathrm{c} 1 \mathrm{ccoc} 2 \mathrm{c} 1 \mathrm{occc} 2=\mathrm{O}$ & 1.63 & 2.59 & 5.56 & 7.34 & 12.31 & 13.60 & 0.77 & 0.77 \\
\hline S1772 & $\mathrm{c} 1 \mathrm{cn} 2 \mathrm{c}(\mathrm{n} 1) \mathrm{cc}[\mathrm{nH}] 2$ & -4.29 & -3.99 & 8.44 & 6.90 & 23.17 & 18.90 & 0.48 & 0.41 \\
\hline S1773 & $\mathrm{c} 1[\mathrm{nH}] \mathrm{n} 2 \mathrm{c}(\mathrm{c} 1) \mathrm{cnc} 2$ & -3.98 & -3.99 & 8.17 & 6.26 & 22.24 & 17.91 & 0.66 & 0.58 \\
\hline S1774 & $\mathrm{c} 1 \mathrm{nc} 2 \mathrm{n}(\mathrm{c} 1) \mathrm{cc}[\mathrm{nH}] 2$ & -4.28 & -3.99 & 4.31 & 4.14 & 16.81 & 15.14 & 0.35 & 0.37 \\
\hline S1775 & $\mathrm{c} 1 \mathrm{cn} 2 \mathrm{c}([\mathrm{nH}] 1) \mathrm{cnc} 2$ & -3.94 & -3.99 & 6.08 & 5.41 & 20.45 & 17.10 & 0.44 & 0.46 \\
\hline S1776 & {$[\mathrm{nH}] 1 \mathrm{cc} 2 \mathrm{c}(\mathrm{c} 1) \mathrm{nc}[\mathrm{nH}] 2$} & -5.76 & -4.74 & 3.48 & 6.92 & 17.31 & 19.73 & 0.40 & 0.40 \\
\hline S1777 & $\mathrm{c} 1 \mathrm{cn} 2 \mathrm{c}(\mathrm{n} 1) \operatorname{cco} 2$ & -2.76 & -2.39 & 4.55 & 4.23 & 15.28 & 13.50 & 0.50 & 0.50 \\
\hline S1778 & $\mathrm{c} 1 \mathrm{on} 2 \mathrm{c}(\mathrm{c} 1) \mathrm{cnc} 2$ & -2.45 & -2.39 & 4.96 & 4.45 & 15.84 & 13.72 & 0.37 & 0.40 \\
\hline S1781 & o1cc2c(c1)nc[nH]2 & -3.55 & -3.14 & 6.66 & 5.97 & 16.42 & 16.33 & 0.61 & 0.56 \\
\hline
\end{tabular}




\begin{tabular}{|c|c|c|c|c|c|c|c|c|}
\hline S1784 & $\mathrm{c} 1 \mathrm{nc} 2 \mathrm{n}(\mathrm{c} 1) \operatorname{ccs} 2$ & -2.98 & -3.08 & 5.06 & 4.31 & 16.02 & 13.40 & 0.41 \\
\hline S1785 & $\mathrm{c} 1 \mathrm{cn} 2 \mathrm{c}(\mathrm{s} 1) \mathrm{cnc} 2$ & -2.66 & -3.08 & 5.08 & 3.98 & 15.75 & 13.26 & 0.56 \\
\hline S1786 & $\operatorname{s1cc2c}(\mathrm{c} 1) \mathrm{nc}[\mathrm{nH}] 2$ & -4.10 & -3.83 & 6.56 & 5.21 & 16.57 & 15.71 & 0.69 \\
\hline S1787 & $\mathrm{c} 1 \mathrm{cn} 2 \mathrm{c}(\mathrm{n} 1) \operatorname{cn}[\mathrm{nH}] 2$ & -2.30 & -2.16 & 8.00 & 8.05 & 18.57 & 18.56 & 0.53 \\
\hline S1788 & $\mathrm{n} 1 \mathrm{cc} 2 \mathrm{n}(\mathrm{c} 1)[\mathrm{nH}] \mathrm{nc} 2$ & -2.05 & -2.16 & 7.35 & 6.98 & 17.30 & 16.63 & 0.55 \\
\hline S1789 & $\mathrm{c} 1 \mathrm{cn} 2 \mathrm{c}(\mathrm{n} 1)[\mathrm{nH}] \mathrm{nc} 2$ & -2.19 & -2.16 & 4.06 & 5.29 & 12.91 & 14.80 & 0.42 \\
\hline S1790 & $\mathrm{n} 1 \mathrm{cc} 2 \mathrm{n}(\mathrm{c} 1) \operatorname{cn}[\mathrm{nH}] 2$ & -1.94 & -2.16 & 4.68 & 6.12 & 15.04 & 15.82 & 0.51 \\
\hline S1791 & $\mathrm{c} 1 \mathrm{nc} 2 \mathrm{c}([\mathrm{nH}] 1) \mathrm{n}[\mathrm{nH}] \mathrm{c} 2$ & -3.88 & -2.91 & 3.76 & 6.03 & 13.70 & 15.95 & 0.86 \\
\hline S1792 & $\mathrm{c} 1 \mathrm{nc} 2 \mathrm{c}([\mathrm{nH}] 1) \mathrm{c}[\mathrm{nH}] \mathrm{n} 2$ & -3.71 & -2.91 & 6.70 & 9.64 & 20.91 & 22.65 & 0.55 \\
\hline S1797 & c1nc2c([nH]1)noc2 & -1.37 & -1.31 & 7.68 & 6.02 & 15.38 & 13.89 & 0.56 \\
\hline S1798 & c1nc2c([nH]1)con2 & -1.16 & -1.31 & 10.60 & 9.63 & 23.02 & 20.59 & 0.53 \\
\hline S18 & $\mathrm{O}=\mathrm{c} 1 \mathrm{occ}[\mathrm{nH}] 1$ & -0.70 & -1.03 & 6.95 & 6.17 & 17.98 & 16.13 & 0.56 \\
\hline S1801 & $\mathrm{c} 1 \mathrm{cn} 2 \mathrm{c}(\mathrm{n} 1) \mathrm{snc} 2$ & -0.91 & -1.25 & 6.61 & 6.35 & 14.23 & 14.23 & 0.57 \\
\hline S18017 & $\mathrm{O}=\mathrm{c} 1 \mathrm{cn}[\mathrm{nH}] \mathrm{c} 2 \mathrm{c} 1 \mathrm{c}[\mathrm{nH}] \mathrm{c} 2$ & -2.73 & -1.76 & 8.75 & 8.91 & 22.13 & 20.77 & 0.43 \\
\hline S1802 & $\mathrm{n} 1 \mathrm{cc} 2 \mathrm{n}(\mathrm{c} 1) \mathrm{cns} 2$ & -0.65 & -1.25 & 6.59 & 5.59 & 13.72 & 13.16 & 0.51 \\
\hline S18020 & $\mathrm{O}=\mathrm{c} 1 \mathrm{cn}[\mathrm{nH}] \mathrm{c} 2 \mathrm{c} 1 \operatorname{coc} 2$ & -0.55 & -0.16 & 7.19 & 7.10 & 16.86 & 16.35 & 0.49 \\
\hline $\mathbf{S 1 8 0 2 3}$ & $\mathrm{O}=\mathrm{c} 1 \mathrm{cn}[\mathrm{nH}] \mathrm{c} 2 \mathrm{c} 1 \mathrm{csc} 2$ & -1.05 & -0.85 & 6.81 & 6.41 & 16.92 & 15.86 & 0.46 \\
\hline S18026 & $\mathrm{O}=\mathrm{c} 1 \mathrm{cn}[\mathrm{nH}] \mathrm{c} 2 \mathrm{c} 1 \mathrm{n}[\mathrm{nH}] \mathrm{c} 2$ & -1.13 & 0.07 & 10.01 & 11.35 & 23.96 & 23.83 & 0.55 \\
\hline S18027 & $\mathrm{O}=\mathrm{c} 1 \mathrm{cn}[\mathrm{nH}] \mathrm{c} 2 \mathrm{c} 1 \mathrm{c}[\mathrm{nH}] \mathrm{n} 2$ & -0.95 & 0.07 & 8.43 & 7.95 & 18.58 & 16.48 & 0.37 \\
\hline S1803 & $\mathrm{c} 1 \mathrm{nc} 2 \mathrm{c}([\mathrm{nH}] 1) \mathrm{nsc} 2$ & -2.28 & -2.00 & 6.39 & 5.21 & 12.99 & 13.11 & 0.63 \\
\hline S18031 & $\mathrm{O}=\mathrm{c} 1 \mathrm{cn}[\mathrm{nH}] \mathrm{c} 2 \mathrm{c} 1 \operatorname{con} 2$ & 1.56 & 1.67 & 5.26 & 7.09 & 11.16 & 13.39 & 0.59 \\
\hline S1804 & $\mathrm{c} 1 \mathrm{nc} 2 \mathrm{c}([\mathrm{nH}] 1) \operatorname{csn} 2$ & -2.05 & -2.00 & 9.41 & 8.82 & 21.63 & 19.81 & 0.58 \\
\hline S18044 & $\mathrm{O}=\mathrm{c} 1 \mathrm{cn}[\mathrm{nH}] \mathrm{c} 2 \mathrm{c} 1 \mathrm{n}[\mathrm{nH}] \mathrm{n} 2$ & 0.68 & 1.90 & 7.52 & 9.36 & 17.87 & 17.29 & 0.47 \\
\hline S1805 & $\operatorname{clnc} 2 \mathrm{c}([\mathrm{nH}] 1)[\mathrm{nH}] \mathrm{cn} 2$ & -3.22 & -2.91 & 9.30 & 13.54 & 24.82 & 28.05 & 0.67 \\
\hline S1806 & c1nc2c([nH]1)nc[nH]2 & -3.53 & -2.91 & 3.23 & 5.89 & 11.34 & 16.08 & 0.63 \\
\hline S18069 & $\mathrm{O}=\mathrm{c} 1[\mathrm{nH}] \mathrm{cc} 2 \mathrm{c}(\mathrm{c} 1) \mathrm{c}(=\mathrm{O}) \mathrm{cn}[\mathrm{nH}] 2$ & 1.47 & 1.22 & 13.01 & 11.57 & 22.61 & 23.02 & 0.63 \\
\hline S1807 & $\mathrm{c} 1 \mathrm{nc} 2 \mathrm{n}(\mathrm{c} 1)[\mathrm{nH}] \mathrm{cn} 2$ & -2.29 & -2.16 & 11.71 & 11.03 & 26.46 & 23.91 & 0.62 \\
\hline S18070 & $\mathrm{O}=\mathrm{c} 1[\mathrm{nH}] \mathrm{cc} 2 \mathrm{c}(\mathrm{c} 1)[\mathrm{nH}] \mathrm{ncc} 2=\mathrm{O}$ & 1.70 & 1.22 & 7.00 & 8.64 & 14.44 & 16.75 & 0.60 \\
\hline S1808 & $\mathrm{c} 1[\mathrm{nH}] \mathrm{n} 2 \mathrm{c}(\mathrm{n} 1) \mathrm{cnc} 2$ & -2.19 & -2.16 & 11.43 & 9.25 & 23.58 & 20.43 & 0.37 \\
\hline S1809 & $\mathrm{c} 1 \mathrm{cn} 2 \mathrm{c}(\mathrm{n} 1)[\mathrm{nH}] \mathrm{cn} 2$ & -2.71 & -2.16 & 7.67 & 5.80 & 17.36 & 15.37 & 0.60 \\
\hline S1810 & $\mathrm{c} 1 \mathrm{nn} 2 \mathrm{c}([\mathrm{nH}] 1) \mathrm{cnc} 2$ & -2.40 & -2.16 & 9.78 & 7.64 & 22.18 & 18.59 & 0.39 \\
\hline
\end{tabular}




\begin{tabular}{|c|c|c|c|c|c|c|c|c|c|}
\hline S1811 & $\mathrm{c} 1 \mathrm{oc} 2 \mathrm{c}(\mathrm{n} 1) \mathrm{nc}[\mathrm{nH}] 2$ & -1.45 & -1.31 & 8.82 & 8.27 & 19.99 & 19.39 & 0.58 & 0.54 \\
\hline S1812 & $\mathrm{c} 1 \mathrm{nc} 2 \mathrm{c}(\mathrm{o} 1) \mathrm{nc}[\mathrm{nH}] 2$ & -1.50 & -1.31 & 6.65 & 6.07 & 14.46 & 13.86 & 0.49 & 0.52 \\
\hline S1814 & c1on $2 \mathrm{c}(\mathrm{n} 1) \mathrm{cnc} 2$ & -0.69 & -0.56 & 6.83 & 5.90 & 15.50 & 14.08 & 0.34 & 0.36 \\
\hline S1815 & $\operatorname{c} 1 \mathrm{cn} 2 \mathrm{c}(\mathrm{n} 1) \operatorname{ocn} 2$ & -0.76 & -0.56 & 5.86 & 5.30 & 14.03 & 12.40 & 0.39 & 0.42 \\
\hline S1816 & $\mathrm{c} 1 \mathrm{nn} 2 \mathrm{c}(\mathrm{o} 1) \mathrm{cnc} 2$ & -0.67 & -0.56 & 6.88 & 5.44 & 15.96 & 13.23 & 0.64 & 0.60 \\
\hline S1817 & $\mathrm{c} 1 \mathrm{sc} 2 \mathrm{c}(\mathrm{n} 1) \mathrm{nc}[\mathrm{nH}] 2$ & -2.34 & -2.00 & 8.48 & 7.79 & 20.28 & 19.42 & 0.55 & 0.56 \\
\hline S1818 & $\mathrm{c} 1 \mathrm{nc} 2 \mathrm{c}(\mathrm{s} 1) \mathrm{nc}[\mathrm{nH}] 2$ & -2.44 & -2.00 & 5.96 & 5.24 & 13.79 & 13.10 & 0.58 & 0.57 \\
\hline S1821 & $\operatorname{c} 1 \mathrm{cn} 2 \mathrm{c}(\mathrm{n} 1) \operatorname{sen} 2$ & -1.69 & -1.25 & 5.06 & 4.52 & 13.38 & 11.73 & 0.48 & 0.50 \\
\hline S1822 & $\mathrm{c} 1 \mathrm{nn} 2 \mathrm{c}(\mathrm{s} 1) \mathrm{cnc} 2$ & -1.36 & -1.25 & 5.82 & 4.76 & 15.09 & 12.86 & 0.42 & 0.46 \\
\hline S1823 & $\mathrm{c} 1 \mathrm{nc} 2 \mathrm{c}([\mathrm{nH}] 1)[\mathrm{nH}] \mathrm{nn} 2$ & -1.01 & -1.08 & 12.28 & 15.12 & 25.69 & 28.88 & 0.45 & 0.43 \\
\hline S1824 & $\mathrm{c} 1[\mathrm{nH}] \mathrm{c} 2 \mathrm{c}(\mathrm{n} 1)[\mathrm{nH}] \mathrm{nn} 2$ & -1.31 & -1.08 & 7.20 & 7.47 & 16.70 & 16.91 & 0.59 & 0.53 \\
\hline S1825 & $\mathrm{c} 1 \mathrm{cn} 2 \mathrm{c}(\mathrm{n} 1) \mathrm{nn}[\mathrm{nH}] 2$ & -0.24 & -0.33 & 11.74 & 12.80 & 22.51 & 24.95 & 0.48 & 0.47 \\
\hline S1826 & $\mathrm{n} 1 \mathrm{cn} 2 \mathrm{c}(\mathrm{c} 1) \mathrm{nn}[\mathrm{nH}] 2$ & -0.25 & -0.33 & 11.08 & 10.60 & 18.58 & 20.54 & 0.47 & 0.44 \\
\hline S1827 & $\mathrm{c} 1 \mathrm{cn} 2 \mathrm{c}(\mathrm{n} 1)[\mathrm{nH}] \mathrm{nn} 2$ & -0.68 & -0.33 & 5.88 & 7.57 & 11.41 & 16.42 & 0.44 & 0.46 \\
\hline S1828 & $\mathrm{n} 1 \mathrm{cn} 2 \mathrm{c}(\mathrm{c} 1)[\mathrm{nH}] \mathrm{nn} 2$ & -0.49 & -0.33 & 8.66 & 8.98 & 16.70 & 18.70 & 0.75 & 0.77 \\
\hline S1829 & {$[\mathrm{nH}] 1 \mathrm{nc} 2 \mathrm{c}(\mathrm{n} 1) \mathrm{nc}[\mathrm{nH}] 2$} & -1.81 & -1.08 & 7.39 & 7.72 & 17.22 & 16.63 & 0.38 & 0.42 \\
\hline S1831 & $\mathrm{c} 1 \mathrm{nc} 2 \mathrm{c}([\mathrm{nH}] 1) \mathrm{nno2}$ & 0.85 & 0.52 & 11.24 & 8.60 & 18.81 & 16.03 & 0.61 & 0.51 \\
\hline S1834 & c1cn2c(n1)onn2 & 1.49 & 1.27 & 5.23 & 8.03 & 10.13 & 14.79 & 0.53 & 0.48 \\
\hline S1835 & $\mathrm{n} 1 \mathrm{cn} 2 \mathrm{c}(\mathrm{c} 1) \mathrm{onn} 2$ & 1.44 & 1.27 & 5.33 & 7.74 & 9.86 & 14.69 & 0.71 & 0.70 \\
\hline S18354 & $\mathrm{O}=\mathrm{c} 1[\mathrm{nH}] \mathrm{cc} 2 \mathrm{c}(\mathrm{c} 1) \mathrm{c}(=\mathrm{O}) \mathrm{cns} 2$ & 2.15 & 2.13 & 10.86 & 8.56 & 18.84 & 16.90 & 0.46 & 0.42 \\
\hline S1836 & $\mathrm{c} 1 \mathrm{nc} 2 \mathrm{c}([\mathrm{nH}] 1) \mathrm{snn} 2$ & -0.15 & -0.17 & 11.69 & 10.26 & 21.85 & 21.42 & 0.46 & 0.44 \\
\hline S1837 & $\mathrm{c} 1 \mathrm{nc} 2 \mathrm{c}([\mathrm{nH}] 1) \mathrm{nns} 2$ & -0.23 & -0.17 & 9.47 & 7.71 & 17.03 & 15.10 & 0.47 & 0.53 \\
\hline S1840 & $\mathrm{c} 1 \mathrm{cn} 2 \mathrm{c}(\mathrm{n} 1) \operatorname{snn} 2$ & 0.31 & 0.58 & 3.25 & 7.19 & 7.88 & 13.95 & 0.64 & 0.64 \\
\hline S1841 & $\mathrm{n} 1 \mathrm{cn} 2 \mathrm{c}(\mathrm{c} 1) \operatorname{snn} 2$ & 0.56 & 0.58 & 3.39 & 7.01 & 8.81 & 14.15 & 0.56 & 0.48 \\
\hline S18429 & $\mathrm{O}=\mathrm{c} 1[\mathrm{nH}] \mathrm{cc} 2 \mathrm{c}(\mathrm{n} 1) \mathrm{scn} \mathrm{c} 2=\mathrm{O}$ & 3.54 & 3.96 & 10.81 & 11.14 & 18.21 & 18.90 & 0.49 & 0.48 \\
\hline S1845 & $\mathrm{o} 1 \mathrm{nc} 2 \mathrm{c}(\mathrm{n} 1) \mathrm{nc}[\mathrm{nH}] 2$ & 1.05 & 0.52 & 12.13 & 8.67 & 20.47 & 15.90 & 0.40 & 0.43 \\
\hline S1846 & $\operatorname{s} 1 \mathrm{nc} 2 \mathrm{c}(\mathrm{n} 1) \mathrm{nc}[\mathrm{nH}] 2$ & -0.21 & -0.17 & 9.59 & 7.79 & 17.69 & 14.95 & 0.45 & 0.53 \\
\hline S1847 & $\mathrm{O}=\mathrm{c} 1[\mathrm{nH}] \mathrm{c} 2 \mathrm{c}([\mathrm{nH}] 1) \mathrm{nc}[\mathrm{nH}] 2$ & -1.64 & -2.14 & 9.93 & 8.93 & 20.80 & 21.08 & 0.47 & 0.44 \\
\hline S18476 & $\mathrm{O}=\mathrm{c} 1 \mathrm{nn}[\mathrm{nH}] \mathrm{c} 2 \mathrm{c} 1 \mathrm{c}[\mathrm{nH}] \mathrm{c} 2$ & -0.81 & 0.07 & 11.25 & 13.94 & 26.54 & 28.77 & 0.58 & 0.61 \\
\hline S18479 & $\mathrm{O}=\mathrm{c} 1 \mathrm{nn}[\mathrm{nH}] \mathrm{c} 2 \mathrm{c} 1 \operatorname{coc} 2$ & 1.28 & 1.67 & 9.93 & 11.32 & 21.34 & 23.21 & 0.59 & 0.58 \\
\hline S18482 & $\mathrm{O}=\mathrm{c} 1 \mathrm{nn}[\mathrm{nH}] \mathrm{c} 2 \mathrm{c} 1 \mathrm{csc} 2$ & 0.81 & 0.98 & 9.51 & 10.67 & 21.46 & 22.86 & 0.45 & 0.41 \\
\hline
\end{tabular}




\begin{tabular}{|c|c|c|c|c|c|c|c|c|c|}
\hline S18485 & $\mathrm{O}=\mathrm{c} 1 \mathrm{nn}[\mathrm{nH}] \mathrm{c} 2 \mathrm{c} 1 \mathrm{n}[\mathrm{nH}] \mathrm{c} 2$ & 0.78 & 1.90 & 12.67 & 16.53 & 27.24 & 32.17 & 0.69 & 0.70 \\
\hline S18486 & $\mathrm{O}=\mathrm{c} 1 \mathrm{nn}[\mathrm{nH}] \mathrm{c} 2 \mathrm{c} 1 \mathrm{c}[\mathrm{nH}] \mathrm{n} 2$ & 0.92 & 1.90 & 10.93 & 12.47 & 22.95 & 23.36 & 0.37 & 0.34 \\
\hline S18490 & $\mathrm{O}=\mathrm{c} 1 \mathrm{nn}[\mathrm{nH}] \mathrm{c} 2 \mathrm{c} 1 \operatorname{con} 2$ & 3.34 & 3.50 & 8.11 & 10.80 & 15.61 & 19.13 & 0.62 & 0.69 \\
\hline S1862 & $\operatorname{c} 1 \mathrm{ccc} 2 \mathrm{c}(\mathrm{c} 1)[\mathrm{nH}] \mathrm{cn} 2$ & -4.54 & -4.36 & 6.44 & 5.22 & 16.99 & 16.64 & 0.38 & 0.34 \\
\hline S1863 & $\operatorname{c} 1 \operatorname{ccc} 2 n(\mathrm{c} 1) \operatorname{ccn} 2$ & -3.35 & -3.61 & 3.94 & 3.93 & 15.12 & 14.18 & 0.50 & 0.53 \\
\hline S1864 & $\mathrm{c} 1 \mathrm{ccc} 2 \mathrm{n}(\mathrm{c} 1) \mathrm{cnc} 2$ & -2.88 & -3.61 & 3.60 & 3.93 & 14.38 & 14.18 & 0.60 & 0.62 \\
\hline S1865 & $\mathrm{c} 1 \mathrm{ncc} 2 \mathrm{c}(\mathrm{c} 1)[\mathrm{nH}] \mathrm{cn} 2$ & -2.47 & -2.53 & 9.24 & 7.57 & 19.21 & 18.52 & 0.48 & 0.55 \\
\hline S1866 & $\mathrm{c} 1 \mathrm{ncc} 2 \mathrm{c}(\mathrm{c} 1) \mathrm{nc}[\mathrm{nH}] 2$ & -2.55 & -2.53 & 8.76 & 6.16 & 17.24 & 15.90 & 0.56 & 0.51 \\
\hline S1867 & $\mathrm{c} 1 \mathrm{cnc} 2 \mathrm{c}(\mathrm{c} 1) \mathrm{nc}[\mathrm{nH}] 2$ & -3.02 & -2.53 & 5.11 & 4.88 & 12.67 & 13.76 & 0.52 & 0.63 \\
\hline S1868 & $\mathrm{c} 1 \mathrm{cnc} 2 \mathrm{c}(\mathrm{c} 1)[\mathrm{nH}] \mathrm{cn} 2$ & -2.72 & -2.53 & 8.82 & 8.77 & 22.36 & 20.97 & 0.41 & 0.37 \\
\hline S1869 & $\mathrm{n} 1 \mathrm{ccn} 2 \mathrm{c}(\mathrm{c} 1) \mathrm{ncc} 2$ & -1.37 & -1.78 & 6.08 & 6.18 & 13.96 & 15.81 & 0.51 & 0.46 \\
\hline S1870 & $\mathrm{n} 1 \mathrm{ccn} 2 \mathrm{c}(\mathrm{c} 1) \mathrm{cnc} 2$ & -0.96 & -1.78 & 5.47 & 5.49 & 12.55 & 14.28 & 0.54 & 0.53 \\
\hline S1871 & $\mathrm{c} 1 \mathrm{ncn} 2 \mathrm{c}(\mathrm{c} 1) \mathrm{ncc} 2$ & -1.42 & -1.78 & 4.78 & 5.31 & 12.60 & 14.07 & 0.55 & 0.58 \\
\hline S1872 & $\mathrm{c} 1 \mathrm{ncn} 2 \mathrm{c}(\mathrm{c} 1) \mathrm{cnc} 2$ & -0.99 & -1.78 & 4.40 & 5.29 & 11.86 & 14.01 & 0.56 & 0.61 \\
\hline S1873 & $\mathrm{c} 1 \mathrm{cnn} 2 \mathrm{c}(\mathrm{c} 1) \mathrm{ncc} 2$ & -2.16 & -1.78 & 3.86 & 4.32 & 12.39 & 12.53 & 0.38 & 0.42 \\
\hline S1874 & $\mathrm{c} 1 \mathrm{cnn} 2 \mathrm{c}(\mathrm{c} 1) \mathrm{cnc} 2$ & -1.65 & -1.78 & 4.79 & 5.08 & 14.35 & 14.19 & 0.67 & 0.65 \\
\hline S1875 & $\mathrm{c} 1 \mathrm{ccn} 2 \mathrm{c}(\mathrm{n} 1) \mathrm{ncc} 2$ & -1.58 & -1.78 & 7.35 & 6.90 & 20.30 & 17.56 & 0.34 & 0.38 \\
\hline S1876 & $\mathrm{c} 1 \mathrm{ccn} 2 \mathrm{c}(\mathrm{n} 1) \mathrm{cnc} 2$ & -1.35 & -1.78 & 7.02 & 5.82 & 16.66 & 15.19 & 0.48 & 0.53 \\
\hline S1877 & $\mathrm{c} 1 \mathrm{nnc} 2 \mathrm{c}(\mathrm{c} 1)[\mathrm{nH}] \mathrm{cn} 2$ & -0.52 & -0.70 & 12.19 & 12.14 & 26.13 & 25.09 & 0.71 & 0.72 \\
\hline S1878 & $\mathrm{c} 1 \mathrm{nnc} 2 \mathrm{c}(\mathrm{c} 1) \mathrm{nc}[\mathrm{nH}] 2$ & -0.91 & -0.70 & 7.94 & 6.84 & 17.29 & 15.27 & 0.37 & 0.38 \\
\hline S1879 & $\mathrm{n} 1 \mathrm{ccn} 2 \mathrm{c}(\mathrm{n} 1) \mathrm{ncc} 2$ & 0.46 & 0.05 & 9.98 & 10.17 & 20.71 & 21.44 & 0.41 & 0.43 \\
\hline S1880 & $\mathrm{n} 1 \mathrm{ccn} 2 \mathrm{c}(\mathrm{n} 1) \mathrm{cnc} 2$ & 0.59 & 0.05 & 9.22 & 8.40 & 16.21 & 17.55 & 0.49 & 0.52 \\
\hline S1882 & $\mathrm{c} 1 \mathrm{nnn} 2 \mathrm{c}(\mathrm{c} 1) \mathrm{cnc} 2$ & 0.18 & 0.05 & 6.14 & 7.46 & 12.29 & 16.28 & 0.36 & 0.37 \\
\hline S1883 & $\mathrm{n} 1 \mathrm{ncc} 2 \mathrm{c}(\mathrm{c} 1)[\mathrm{nH}] \mathrm{cn} 2$ & -0.29 & -0.70 & 12.03 & 9.53 & 23.96 & 20.02 & 0.46 & 0.47 \\
\hline S1884 & $\mathrm{n} 1 \mathrm{ncc} 2 \mathrm{n}(\mathrm{c} 1) \operatorname{ccn} 2$ & 0.80 & 0.05 & 7.93 & 8.59 & 15.51 & 17.95 & 0.64 & 0.65 \\
\hline S1885 & $\mathrm{n} 1 \mathrm{ncc} 2 \mathrm{n}(\mathrm{c} 1) \mathrm{cnc} 2$ & 1.18 & 0.05 & 7.24 & 7.87 & 14.22 & 16.37 & 0.51 & 0.51 \\
\hline S1886 & $\mathrm{O}=\mathrm{c} 1[\mathrm{nH}] \mathrm{ccc} 2 \mathrm{c} 1 \mathrm{nc}[\mathrm{nH}] 2$ & -2.36 & -1.76 & 9.04 & 10.03 & 25.45 & 24.42 & 0.71 & 0.70 \\
\hline S1887 & $\mathrm{O}=\mathrm{c} 1[\mathrm{nH}] \mathrm{ccc} 2 \mathrm{c} 1[\mathrm{nH}] \mathrm{cn} 2$ & -2.11 & -1.76 & 3.94 & 7.35 & 12.06 & 16.40 & 0.59 & 0.58 \\
\hline S1888 & $\mathrm{O}=\mathrm{c} 1 \mathrm{ccc} 2 \mathrm{c}([\mathrm{nH}] 1)[\mathrm{nH}] \mathrm{cn} 2$ & -1.12 & -1.76 & 11.59 & 10.18 & 22.52 & 21.06 & 0.39 & 0.41 \\
\hline S1889 & $\mathrm{O}=\mathrm{c} 1 \mathrm{ccc} 2 \mathrm{c}([\mathrm{nH}] 1) \mathrm{nc}[\mathrm{nH}] 2$ & -1.32 & -1.76 & 10.84 & 7.39 & 24.09 & 19.07 & 0.89 & 0.95 \\
\hline S1890 & $\mathrm{O}=\mathrm{c} 1[\mathrm{nH}] \mathrm{ccn} 2 \mathrm{c} 1 \mathrm{ncc} 2$ & -1.35 & -1.01 & 7.96 & 8.22 & 21.97 & 20.83 & 0.51 & 0.51 \\
\hline
\end{tabular}




\begin{tabular}{|c|c|c|c|c|c|c|c|c|c|}
\hline S1891 & $\mathrm{O}=\mathrm{c} 1[\mathrm{nH}] \operatorname{ccn} 2 \mathrm{c} 1 \mathrm{cnc} 2$ & -0.83 & -1.01 & 8.21 & 7.82 & 17.64 & 18.72 & 0.57 & 0.50 \\
\hline S1892 & $\mathrm{O}=\mathrm{c} 1[\mathrm{nH}] \operatorname{ccc} 2 \mathrm{n} 1 \mathrm{ccn} 2$ & -1.29 & -1.01 & 7.82 & 6.36 & 15.97 & 15.15 & 0.64 & 0.63 \\
\hline S1893 & $\mathrm{O}=\mathrm{c} 1[\mathrm{nH}] \operatorname{ccc} 2 \mathrm{n} 1 \mathrm{cnc} 2$ & -0.83 & -1.01 & 8.08 & 7.22 & 17.44 & 17.52 & 0.62 & 0.58 \\
\hline S1896 & $\mathrm{O}=\mathrm{c} 1 \mathrm{ccn} 2 \mathrm{c}([\mathrm{nH}] 1) \mathrm{ncc} 2$ & -0.06 & -1.01 & 7.82 & 6.63 & 16.37 & 17.00 & 0.53 & 0.50 \\
\hline S1897 & $\mathrm{O}=\mathrm{c} 1 \mathrm{ccn} 2 \mathrm{c}([\mathrm{nH}] 1) \mathrm{cnc} 2$ & 0.43 & -1.01 & 7.60 & 7.07 & 14.95 & 16.73 & 0.34 & 0.37 \\
\hline S19238 & $\mathrm{O}=\mathrm{c} 1 \mathrm{oc} 2 \operatorname{cocc} 2 \mathrm{c}(=\mathrm{O})[\mathrm{nH}] 1$ & 2.27 & 2.21 & 5.67 & 7.04 & 11.57 & 12.35 & 0.55 & 0.54 \\
\hline S19239 & $\mathrm{O}=\mathrm{c} 1 \mathrm{oc} 2 \operatorname{cscc} 2 \mathrm{c}(=\mathrm{O})[\mathrm{nH}] 1$ & 1.63 & 1.52 & 4.89 & 6.29 & 11.26 & 11.80 & 0.51 & 0.50 \\
\hline S19243 & $\mathrm{O}=\mathrm{c} 1 \mathrm{oc} 2 \operatorname{nocc} 2 \mathrm{c}(=\mathrm{O})[\mathrm{nH}] 1$ & 4.51 & 4.04 & 9.97 & 9.24 & 14.08 & 12.69 & 0.59 & 0.55 \\
\hline S1928 & $\mathrm{c} 1 \mathrm{ncc} 2 \mathrm{c}(\mathrm{n} 1) \mathrm{nc}[\mathrm{nH}] 2$ & -0.70 & -0.70 & 11.29 & 9.11 & 21.82 & 18.92 & 0.83 & 0.84 \\
\hline S1929 & $\mathrm{c} 1 \mathrm{ncc} 2 \mathrm{c}(\mathrm{n} 1)[\mathrm{nH}] \mathrm{cn} 2$ & -0.93 & -0.70 & 8.23 & 6.64 & 14.85 & 14.35 & 0.38 & 0.41 \\
\hline S19294 & $\mathrm{O}=\mathrm{c} 1 \mathrm{sc} 2 \operatorname{coc} 2 \mathrm{c}(=\mathrm{O})[\mathrm{nH}] 1$ & 1.36 & 1.52 & 5.44 & 6.22 & 11.18 & 11.60 & 0.48 & 0.50 \\
\hline S1930 & $\mathrm{c} 1 \mathrm{ncn} 2 \mathrm{c}(\mathrm{n} 1) \mathrm{ncc} 2$ & 0.40 & 0.05 & 8.31 & 7.69 & 17.90 & 16.16 & 0.45 & 0.41 \\
\hline S1931 & $\mathrm{c} 1 \mathrm{ncn} 2 \mathrm{c}(\mathrm{n} 1) \mathrm{cnc} 2$ & 0.58 & 0.05 & 7.95 & 6.59 & 14.10 & 13.73 & 0.70 & 0.72 \\
\hline S1932 & $\mathrm{n} 1 \mathrm{cnn} 2 \mathrm{c}(\mathrm{c} 1) \mathrm{ncc} 2$ & -0.15 & 0.05 & 2.60 & 5.98 & 7.67 & 12.86 & 0.30 & 0.29 \\
\hline S1933 & $\mathrm{n} 1 \mathrm{cnn} 2 \mathrm{c}(\mathrm{c} 1) \mathrm{cnc} 2$ & 0.30 & 0.05 & 4.19 & 6.05 & 9.89 & 13.00 & 0.67 & 0.69 \\
\hline S1934 & $\mathrm{c} 1 \mathrm{nc} 2 \mathrm{c}([\mathrm{nH}] 1) \mathrm{cnnn} 2$ & 1.62 & 1.13 & 14.88 & 13.50 & 27.24 & 25.30 & 0.79 & 0.84 \\
\hline S1935 & $\mathrm{c} 1 \mathrm{nc} 2 \mathrm{c}([\mathrm{nH}] 1) \mathrm{nnnc} 2$ & 1.29 & 1.13 & 11.47 & 9.62 & 21.64 & 18.10 & 0.59 & 0.58 \\
\hline S1936 & $\mathrm{n} 1 \mathrm{ncn} 2 \mathrm{c}(\mathrm{n} 1) \mathrm{ncc} 2$ & 2.59 & 1.88 & 11.59 & 11.99 & 19.61 & 22.28 & 0.43 & 0.51 \\
\hline S19363 & $\mathrm{O}=\mathrm{c} 1 \mathrm{ccc} 2 \mathrm{n} 1 \mathrm{n} \csc 2$ & -0.70 & -0.10 & 6.52 & 6.67 & 18.86 & 17.70 & 0.42 & 0.45 \\
\hline S1937 & $\mathrm{n} 1 \mathrm{ncn} 2 \mathrm{c}(\mathrm{n} 1) \mathrm{cnc} 2$ & 2.71 & 1.88 & 10.95 & 10.19 & 17.54 & 18.34 & 0.51 & 0.55 \\
\hline S19383 & $\mathrm{O}=\mathrm{c} 1 \mathrm{nc} 2 \mathrm{c}(\mathrm{n} 1) \operatorname{scn}[\mathrm{nH}] 2$ & 3.19 & 2.81 & 10.33 & 8.52 & 21.72 & 15.29 & 0.54 & 0.56 \\
\hline S1939 & $\mathrm{n} 1 \mathrm{nnn} 2 \mathrm{c}(\mathrm{c} 1) \mathrm{cnc} 2$ & 2.33 & 1.88 & 6.95 & 9.45 & 11.98 & 17.33 & 0.49 & 0.52 \\
\hline S1940 & $\mathrm{O}=\mathrm{c} 1[\mathrm{nH}] \mathrm{ncc} 2 \mathrm{c} 1 \mathrm{nc}[\mathrm{nH}] 2$ & -0.46 & 0.07 & 12.10 & 9.17 & 26.08 & 20.09 & 0.27 & 0.28 \\
\hline S1941 & $\mathrm{O}=\mathrm{c} 1[\mathrm{nH}] \mathrm{ncc} 2 \mathrm{c} 1[\mathrm{nH}] \mathrm{cn} 2$ & -0.15 & 0.07 & 7.53 & 7.91 & 14.08 & 14.69 & 0.34 & 0.37 \\
\hline S1942 & $\mathrm{O}=\mathrm{c} 1[\mathrm{nH}] \mathrm{ncn} 2 \mathrm{c} 1 \mathrm{ncc} 2$ & 0.58 & 0.82 & 8.96 & 7.81 & 20.47 & 17.14 & 0.83 & 0.79 \\
\hline S1943 & $\mathrm{O}=\mathrm{c} 1[\mathrm{nH}] \mathrm{ncn} 2 \mathrm{c} 1 \mathrm{cnc} 2$ & 1.03 & 0.82 & 8.65 & 7.38 & 16.29 & 14.97 & 0.47 & 0.48 \\
\hline S1944 & $\mathrm{O}=\mathrm{c} 1[\mathrm{nH}] \mathrm{ncc} 2 \mathrm{n} 1 \mathrm{ccn} 2$ & 0.62 & 0.82 & 4.28 & 6.82 & 8.47 & 13.19 & 0.53 & 0.50 \\
\hline S1945 & $\mathrm{O}=\mathrm{c} 1[\mathrm{nH}] \mathrm{ncc} 2 \mathrm{n} 1 \mathrm{cnc} 2$ & 1.03 & 0.82 & 4.77 & 6.99 & 10.05 & 14.04 & 0.66 & 0.68 \\
\hline S1948 & $\mathrm{O}=\mathrm{c} 1 \mathrm{ncc} 2 \mathrm{c}([\mathrm{nH}] 1) \mathrm{nc}[\mathrm{nH}] 2$ & 0.56 & 0.07 & 13.28 & 10.10 & 28.10 & 22.49 & 0.92 & 0.91 \\
\hline S1949 & $\mathrm{O}=\mathrm{c} 1 \mathrm{ncc} 2 \mathrm{c}([\mathrm{nH}] 1)[\mathrm{nH}] \mathrm{cn} 2$ & 0.85 & 0.07 & 14.47 & 14.31 & 27.77 & 27.12 & 0.74 & 0.75 \\
\hline S1950 & $\mathrm{O}=\mathrm{c} 1 \mathrm{ncn} 2 \mathrm{c}([\mathrm{nH}] 1) \mathrm{ncc} 2$ & 1.78 & 0.82 & 9.14 & 9.79 & 18.86 & 21.07 & 0.56 & 0.66 \\
\hline
\end{tabular}




\begin{tabular}{|c|c|c|c|c|c|c|c|c|c|}
\hline S1951 & $\mathrm{O}=\mathrm{c} 1 \mathrm{ncn} 2 \mathrm{c}([\mathrm{nH}] 1) \mathrm{cnc} 2$ & 2.22 & 0.82 & 9.03 & 10.21 & 17.87 & 20.73 & 0.43 & 0.50 \\
\hline S19590 & $\mathrm{O}=\mathrm{c} 1[\mathrm{nH}] \mathrm{nc} 2 \mathrm{n}(\mathrm{c} 1=\mathrm{O}) \mathrm{cccc} 2$ & 1.31 & 1.97 & 6.71 & 8.58 & 17.64 & 17.80 & 0.49 & 0.49 \\
\hline S19591 & $\mathrm{O}=\mathrm{c} 1[\mathrm{nH}] \mathrm{nc} 2 \mathrm{n}(\mathrm{c} 1=\mathrm{O}) \mathrm{ccnc} 2$ & 3.31 & 3.80 & 9.14 & 10.40 & 17.15 & 17.66 & 0.87 & 0.82 \\
\hline S19592 & $\mathrm{O}=\mathrm{c} 1[\mathrm{nH}] \mathrm{nc} 2 \mathrm{n}(\mathrm{c} 1=\mathrm{O}) \mathrm{cncc} 2$ & 3.27 & 3.80 & 9.09 & 10.87 & 17.30 & 19.32 & 0.47 & 0.45 \\
\hline S19593 & $\mathrm{O}=\mathrm{c} 1[\mathrm{nH}] \mathrm{nc} 2 \mathrm{n}(\mathrm{c} 1=\mathrm{O}) \mathrm{nccc} 2$ & 2.09 & 3.80 & 9.60 & 11.03 & 21.00 & 21.43 & 0.78 & 0.80 \\
\hline S19611 & $\mathrm{O}=\mathrm{c} 1[\mathrm{nH}] \mathrm{nc} 2 \mathrm{n}(\mathrm{c} 1=\mathrm{O}) \mathrm{ncnc} 2$ & 4.12 & 5.63 & 11.13 & 12.25 & 19.73 & 19.99 & 0.76 & 0.76 \\
\hline S19645 & $\mathrm{O}=\mathrm{c} 1[\mathrm{nH}] \mathrm{nc} 2 \mathrm{n}(\mathrm{c} 1=\mathrm{O}) \mathrm{nccn} 2$ & 3.51 & 5.63 & 11.93 & 11.41 & 21.50 & 18.68 & 0.57 & 0.58 \\
\hline S19848 & $\mathrm{O}=\mathrm{c} 1 \mathrm{ccc} 2 \mathrm{n} 1 \mathrm{nc}[\mathrm{nH}] \mathrm{n} 2$ & -0.51 & 0.82 & 8.43 & 8.27 & 21.28 & 18.17 & 0.37 & 0.33 \\
\hline S19850 & $\mathrm{O}=\mathrm{c} 1 \mathrm{ncc} 2 \mathrm{n} 1 \mathrm{nc}[\mathrm{nH}] \mathrm{n} 2$ & 1.67 & 2.65 & 11.55 & 11.66 & 24.27 & 22.33 & 0.56 & 0.60 \\
\hline S19852 & $\mathrm{O}=\mathrm{c} 1 \mathrm{cnc} 2 \mathrm{n} 1 \mathrm{nc}[\mathrm{nH}] \mathrm{n} 2$ & 1.43 & 2.65 & 10.56 & 9.38 & 20.49 & 16.74 & 0.52 & 0.56 \\
\hline S1988 & $\mathrm{O}=\mathrm{c} 1 \mathrm{cnc} 2 \mathrm{c}([\mathrm{nH}] 1)[\mathrm{nH}] \mathrm{cn} 2$ & 0.61 & 0.07 & 13.82 & 14.22 & 22.92 & 25.34 & 0.95 & .95 \\
\hline S1989 & $\mathrm{O}=\mathrm{c} 1 \mathrm{cnc} 2 \mathrm{c}([\mathrm{nH}] 1) \mathrm{nc}[\mathrm{nH}] 2$ & 0.14 & 0.07 & 9.24 & 7.54 & 19.26 & 16.14 & 0.50 & 0.52 \\
\hline S1992 & $\mathrm{O}=\mathrm{c} 1 \mathrm{cnn} 2 \mathrm{c}([\mathrm{nH}] 1) \mathrm{ncc} 2$ & 1.13 & 0.82 & 5.49 & 7.52 & 10.58 & 15.30 & 0.58 & 0.59 \\
\hline S1993 & $\mathrm{O}=\mathrm{c} 1 \mathrm{cnn} 2 \mathrm{c}([\mathrm{nH}] 1) \mathrm{cnc} 2$ & 1.63 & 0.82 & 6.32 & 8.71 & 12.30 & 16.69 & 0.80 & 0.81 \\
\hline S1994 & $\mathrm{O}=\mathrm{c} 1 \mathrm{nnc} 2 \mathrm{c}([\mathrm{nH}] 1)[\mathrm{nH}] \mathrm{cn} 2$ & 2.65 & 1.90 & 17.43 & 19.37 & 29.26 & 33.64 & 0.34 & 0.38 \\
\hline S1995 & $\mathrm{O}=\mathrm{c} 1 \mathrm{nnc} 2 \mathrm{c}([\mathrm{nH}] 1) \mathrm{nc}[\mathrm{nH}] 2$ & 2.08 & 1.90 & 12.64 & 11.28 & 25.19 & 21.82 & 0.71 & 0.66 \\
\hline $\mathbf{S 2 0 0 0}$ & $\mathrm{O}=\mathrm{c} 1[\mathrm{nH}] \mathrm{c}(=\mathrm{O}) \mathrm{c} 2 \mathrm{c}([\mathrm{nH}] 1)[\mathrm{nH}] \mathrm{cn} 2$ & 1.06 & 0.84 & 14.85 & 13.54 & 27.13 & 25.49 & 0.47 & 0.50 \\
\hline S2001 & $\mathrm{O}=\mathrm{c} 1[\mathrm{nH}] \mathrm{c}(=\mathrm{O}) \mathrm{c} 2 \mathrm{c}([\mathrm{nH}] 1) \mathrm{nc}[\mathrm{nH}] 2$ & 1.09 & 0.84 & 8.90 & 8.07 & 16.34 & 15.48 & 0.41 & 0.44 \\
\hline S2004 & $\mathrm{O}=\mathrm{c} 1[\mathrm{nH}] \mathrm{c}(=\mathrm{O}) \mathrm{n} 2 \mathrm{c}([\mathrm{nH}] 1) \mathrm{ncc} 2$ & 2.14 & 1.59 & 7.61 & 7.61 & 11.03 & 14.62 & 0.65 & 0.63 \\
\hline S2005 & $\mathrm{O}=\mathrm{c} 1[\mathrm{nH}] \mathrm{c}(=\mathrm{O}) \mathrm{n} 2 \mathrm{c}([\mathrm{nH}] 1) \mathrm{cnc} 2$ & 2.62 & 1.59 & 8.12 & 8.91 & 13.12 & 16.72 & 0.52 & 0.52 \\
\hline S2006 & $\mathrm{O}=\mathrm{c} 1[\mathrm{nH}] \mathrm{c} 2[\mathrm{nH}] \mathrm{cnc} 2[\mathrm{nH}] \mathrm{c} 1=\mathrm{O}$ & 0.99 & 0.84 & 13.82 & 13.74 & 26.74 & 29.37 & 0.69 & 0.70 \\
\hline S2009 & $\mathrm{O}=\mathrm{c} 1 \mathrm{oc}(=\mathrm{O}) \mathrm{c} 2 \mathrm{c}([\mathrm{nH}] 1)[\mathrm{nH}] \mathrm{cn} 2$ & 2.54 & 2.44 & 17.17 & 18.03 & 28.86 & 30.43 & 0.66 & 0.61 \\
\hline S2010 & $\mathrm{O}=\mathrm{c} 1 \mathrm{oc}(=\mathrm{O}) \mathrm{c} 2 \mathrm{c}([\mathrm{nH}] 1) \mathrm{nc}[\mathrm{nH}] 2$ & 2.52 & 2.44 & 11.37 & 10.42 & 20.28 & 17.89 & 0.36 & 0.37 \\
\hline S20299 & $\mathrm{O}=\mathrm{c} 1 \mathrm{cnn} 2 \mathrm{c}(\mathrm{n} 1) \operatorname{scc} 2=\mathrm{O}$ & 3.43 & 4.71 & 10.76 & 10.40 & 20.02 & 17.94 & 0.76 & 0.72 \\
\hline $\mathbf{S 2 0 3 3}$ & $\mathrm{O}=\mathrm{c} 1 \mathrm{cc}[\mathrm{nH}] \mathrm{c} 2 \mathrm{c} 1[\mathrm{nH}] \mathrm{cn} 2$ & -2.19 & -1.76 & 5.66 & 6.95 & 17.97 & 17.36 & 0.70 & 0.66 \\
\hline S2034 & $\mathrm{O}=\mathrm{c} 1 \mathrm{cc}[\mathrm{nH}] \mathrm{c} 2 \mathrm{c} 1 \mathrm{nc}[\mathrm{nH}] 2$ & -2.31 & -1.76 & 13.79 & 15.56 & 33.07 & 33.13 & 0.48 & 0.48 \\
\hline S2035 & $\mathrm{O}=\mathrm{c} 1 \mathrm{cc}[\mathrm{nH}] \mathrm{c} 2 \mathrm{n} 1 \mathrm{ccn} 2$ & -1.30 & -1.01 & 8.82 & 7.10 & 17.82 & 17.36 & 0.49 & 0.49 \\
\hline S2036 & $\mathrm{O}=\mathrm{c} 1 \mathrm{cc}[\mathrm{nH}] \mathrm{c} 2 \mathrm{n} 1 \mathrm{cnc} 2$ & -0.83 & -1.01 & 11.14 & 9.13 & 20.65 & 21.56 & 0.41 & 0.44 \\
\hline S2039 & $\mathrm{O}=\mathrm{c} 1 \mathrm{nc}[\mathrm{nH}] \mathrm{c} 2 \mathrm{c} 1[\mathrm{nH}] \mathrm{cn} 2$ & -0.28 & 0.07 & 7.64 & 9.66 & 21.14 & 20.79 & 0.39 & 0.40 \\
\hline S2040 & $\mathrm{O}=\mathrm{c} 1 \mathrm{nc}[\mathrm{nH}] \mathrm{c} 2 \mathrm{c} 1 \mathrm{nc}[\mathrm{nH}] 2$ & -0.40 & 0.07 & 16.09 & 19.69 & 35.02 & 39.18 & 0.72 & 0.60 \\
\hline S2041 & $\mathrm{O}=\mathrm{c} 1 \mathrm{nc}[\mathrm{nH}] \mathrm{c} 2 \mathrm{n} 1 \mathrm{ccn} 2$ & 0.54 & 0.82 & 10.93 & 10.27 & 20.70 & 21.43 & 0.77 & 0.76 \\
\hline
\end{tabular}




\begin{tabular}{|c|c|c|c|c|c|c|c|c|c|}
\hline S2045 & $\mathrm{c} 1 \mathrm{cnc} 2 \mathrm{c}(\mathrm{n} 1)[\mathrm{nH}] \mathrm{cn} 2$ & -1.19 & -0.70 & 7.87 & 7.40 & 17.60 & 15.84 & 0.47 & 0.44 \\
\hline S2046 & $\mathrm{c} 1 \mathrm{cnc} 2 \mathrm{n}(\mathrm{n} 1) \operatorname{ccn} 2$ & -0.42 & 0.05 & 5.82 & 6.27 & 15.63 & 13.66 & 0.52 & 0.58 \\
\hline S2047 & $\mathrm{c} 1 \mathrm{cnc} 2 \mathrm{n}(\mathrm{n} 1) \mathrm{cnc} 2$ & -0.16 & 0.05 & 6.64 & 5.95 & 14.60 & 12.96 & 0.33 & 0.36 \\
\hline S2048 & $\mathrm{c} 1 \mathrm{nnc} 2 \mathrm{c}(\mathrm{n} 1) \mathrm{nc}[\mathrm{nH}] 2$ & 0.98 & 1.13 & 11.08 & 8.77 & 17.55 & 16.05 & 0.51 & 0.49 \\
\hline S2049 & $\mathrm{c} 1 \mathrm{nnc} 2 \mathrm{c}(\mathrm{n} 1)[\mathrm{nH}] \mathrm{cn} 2$ & 1.07 & 1.13 & 11.66 & 10.18 & 21.81 & 18.67 & 0.38 & 0.41 \\
\hline S20516 & $\mathrm{O}=\mathrm{c} 1 \mathrm{ccc} 2 \mathrm{n} 1 \mathrm{ncsn} 2$ & 0.68 & 1.73 & 4.94 & 7.01 & 13.92 & 14.27 & 0.42 & 0.49 \\
\hline S2052 & $\mathrm{n} 1 \mathrm{cnn} 2 \mathrm{c}(\mathrm{n} 1) \mathrm{ncc} 2$ & 1.67 & 1.88 & 6.06 & 8.95 & 12.93 & 16.24 & 0.60 & 0.64 \\
\hline $\mathbf{S 2 0 5 3}$ & $\mathrm{n} 1 \mathrm{cnn} 2 \mathrm{c}(\mathrm{n} 1) \mathrm{cnc} 2$ & 1.85 & 1.88 & 5.72 & 7.94 & 9.12 & 14.02 & 0.48 & 0.43 \\
\hline S2054 & $\mathrm{O}=\mathrm{c} 1[\mathrm{nH}] \mathrm{cnc} 2 \mathrm{c} 1[\mathrm{nH}] \mathrm{cn} 2$ & -0.36 & 0.07 & 6.01 & 10.43 & 15.36 & 18.60 & 0.42 & 0.39 \\
\hline S2055 & $\mathrm{O}=\mathrm{c} 1[\mathrm{nH}] \mathrm{cnc} 2 \mathrm{c} 1 \mathrm{nc}[\mathrm{nH}] 2$ & -0.90 & 0.07 & 7.18 & 9.23 & 20.37 & 19.42 & 0.43 & 0.42 \\
\hline $\mathbf{S 2 0 5 6}$ & $\mathrm{O}=\mathrm{c} 1[\mathrm{nH}] \mathrm{cnc} 2 \mathrm{n} 1 \mathrm{ccn} 2$ & 0.48 & 0.82 & 10.39 & 8.87 & 19.92 & 16.40 & 0.82 & 0.75 \\
\hline S2057 & $\mathrm{O}=\mathrm{c} 1[\mathrm{nH}] \mathrm{cnc} 2 \mathrm{n} 1 \mathrm{cnc} 2$ & 0.74 & 0.82 & 10.51 & 8.65 & 18.67 & 16.42 & 0.51 & 0.59 \\
\hline $\mathbf{S 2 0 5 8}$ & $\mathrm{O}=\mathrm{c} 1[\mathrm{nH}] \mathrm{cnn} 2 \mathrm{c} 1 \mathrm{ncc} 2$ & -0.09 & 0.82 & 9.86 & 8.15 & 20.74 & 17.05 & 0.86 & 0.80 \\
\hline S2059 & $\mathrm{O}=\mathrm{c} 1[\mathrm{nH}] \mathrm{cnn} 2 \mathrm{c} 1 \mathrm{cnc} 2$ & 0.46 & 0.82 & 10.20 & 8.50 & 17.99 & 16.61 & 0.44 & 0.52 \\
\hline S2084 & $\mathrm{n} 1 \mathrm{nnc} 2 \mathrm{c}(\mathrm{n} 1)[\mathrm{nH}] \mathrm{cn} 2$ & 3.30 & 2.96 & 15.02 & 12.57 & 24.34 & 21.12 & 0.39 & 0.44 \\
\hline S2085 & $\mathrm{n} 1 \mathrm{nnc} 2 \mathrm{n}(\mathrm{n} 1) \mathrm{ccn} 2$ & 3.73 & 3.71 & 9.12 & 11.79 & 13.94 & 19.34 & 0.52 & 0.49 \\
\hline S2086 & $\mathrm{n} 1 \mathrm{nnc} 2 \mathrm{n}(\mathrm{n} 1) \mathrm{cnc} 2$ & 3.83 & 3.71 & 8.89 & 10.75 & 12.35 & 17.05 & 0.41 & 0.46 \\
\hline $\mathbf{S 2 0 9 3}$ & $\mathrm{O}=\mathrm{c} 1[\mathrm{nH}] \mathrm{nnc} 2 \mathrm{c} 1 \mathrm{nc}[\mathrm{nH}] 2$ & 0.98 & 1.90 & 10.53 & 9.39 & 20.87 & 17.33 & 0.51 & 0.54 \\
\hline S2094 & $\mathrm{O}=\mathrm{c} 1[\mathrm{nH}] \mathrm{nnc} 2 \mathrm{c} 1[\mathrm{nH}] \mathrm{cn} 2$ & 1.58 & 1.90 & 10.03 & 12.01 & 17.53 & 19.14 & 0.61 & 0.64 \\
\hline S2097 & $\mathrm{O}=\mathrm{c} 1[\mathrm{nH}] \mathrm{nnc} 2 \mathrm{n} 1 \mathrm{ccn} 2$ & 2.32 & 2.65 & 7.70 & 10.35 & 13.82 & 16.70 & 0.57 & 0.55 \\
\hline S2098 & $\mathrm{O}=\mathrm{c} 1[\mathrm{nH}] \mathrm{nnc} 2 \mathrm{n} 1 \mathrm{cnc} 2$ & 2.52 & 2.65 & 7.71 & 9.44 & 11.39 & 15.18 & 0.43 & 0.38 \\
\hline S2126 & $\mathrm{O}=\mathrm{c} 1 \mathrm{ccoc} 2 \mathrm{n} 1 \mathrm{cnc} 2$ & 0.64 & 0.59 & 8.02 & 6.85 & 15.58 & 15.59 & 0.48 & 0.46 \\
\hline S2139 & $\mathrm{O}=\mathrm{c} 1 \mathrm{ccsc} 2 \mathrm{n} 1 \mathrm{ccn} 2$ & -0.42 & -0.10 & 6.36 & 5.67 & 12.24 & 13.09 & 0.51 & 0.46 \\
\hline $\mathbf{S 2 1 4 0}$ & $\mathrm{O}=\mathrm{c} 1 \operatorname{cssc} 2 \mathrm{n} 1 \mathrm{cnc} 2$ & 0.05 & -0.10 & 7.10 & 6.18 & 14.62 & 15.29 & 0.71 & 0.65 \\
\hline S2141 & $\mathrm{O}=\mathrm{c} 1 \mathrm{cn}[\mathrm{nH}] \mathrm{c} 2 \mathrm{c} 1[\mathrm{nH}] \mathrm{cn} 2$ & -0.28 & 0.07 & 7.43 & 7.50 & 16.90 & 15.66 & 0.70 & 0.64 \\
\hline S2142 & $\mathrm{O}=\mathrm{c} 1 \mathrm{cn}[\mathrm{nH}] \mathrm{c} 2 \mathrm{c} 1 \mathrm{nc}[\mathrm{nH}] 2$ & -0.48 & 0.07 & 14.77 & 14.70 & 30.56 & 28.79 & 0.63 & 0.66 \\
\hline S2143 & $\mathrm{O}=\mathrm{c} 1 \mathrm{cn}[\mathrm{nH}] \mathrm{c} 2 \mathrm{n} 1 \mathrm{ccn} 2$ & 0.56 & 0.82 & 5.12 & 7.56 & 10.80 & 15.41 & 0.29 & 0.31 \\
\hline S2144 & $\mathrm{O}=\mathrm{c} 1 \mathrm{cn}[\mathrm{nH}] \mathrm{c} 2 \mathrm{n} 1 \mathrm{cnc} 2$ & 0.94 & 0.82 & 8.68 & 8.90 & 14.96 & 18.07 & 0.55 & 0.49 \\
\hline S2155 & $\mathrm{O}=\mathrm{c} 1 \mathrm{cnsc} 2 \mathrm{n} 1 \mathrm{ccn} 2$ & 1.43 & 1.73 & 6.09 & 7.57 & 9.79 & 13.03 & 0.38 & 0.41 \\
\hline $\mathbf{S 2 1 5 6}$ & $\mathrm{O}=\mathrm{c} 1 \mathrm{cnsc} 2 \mathrm{n} 1 \mathrm{cnc} 2$ & 1.84 & 1.73 & 6.57 & 7.39 & 11.42 & 13.70 & 0.58 & 0.53 \\
\hline S2165 & $\mathrm{O}=\mathrm{c} 1 \mathrm{nn}[\mathrm{nH}] \mathrm{c} 2 \mathrm{c} 1[\mathrm{nH}] \mathrm{cn} 2$ & 1.61 & 1.90 & 10.13 & 11.24 & 21.53 & 21.33 & 0.35 & 0.37 \\
\hline
\end{tabular}




\begin{tabular}{|c|c|c|c|c|c|c|c|c|c|}
\hline S2166 & $\mathrm{O}=\mathrm{c} 1 \mathrm{nn}[\mathrm{nH}] \mathrm{c} 2 \mathrm{c} 1 \mathrm{nc}[\mathrm{nH}] 2$ & 1.33 & 1.90 & 17.35 & 19.85 & 33.13 & 37.09 & 0.42 & 0.41 \\
\hline S21724 & $\mathrm{O}=\mathrm{c} 1 \mathrm{cnnc} 2 \mathrm{n} 1 \mathrm{nc}[\mathrm{nH}] 2$ & 1.90 & 2.65 & 14.06 & 10.74 & 23.44 & 19.42 & 0.49 & 0.49 \\
\hline $\mathbf{S 2 1 7 2 6}$ & $\mathrm{O}=\mathrm{c} 1 \mathrm{cnnc} 2 \mathrm{n} 1 \mathrm{nco} 2$ & 3.81 & 4.25 & 11.96 & 9.51 & 17.64 & 14.94 & 0.49 & 0.45 \\
\hline S21728 & $\mathrm{O}=\mathrm{c} 1 \mathrm{cnnc} 2 \mathrm{n} 1 \mathrm{ncs} 2$ & 2.75 & 3.56 & 10.53 & 8.78 & 17.37 & 14.46 & 0.44 & 0.40 \\
\hline S21744 & $\mathrm{O}=\mathrm{c} 1 \mathrm{cnn} \operatorname{c} 2 \mathrm{n} 1 \mathrm{nccc} 2$ & 2.08 & 3.03 & 10.42 & 8.91 & 18.74 & 15.80 & 0.44 & 0.37 \\
\hline $\mathbf{S 2 1 7 6 2}$ & $\mathrm{O}=\mathrm{c} 1 \mathrm{cnn} \mathrm{c} 2 \mathrm{n} 1 \mathrm{ncnc} 2$ & 4.12 & 4.86 & 10.84 & 10.13 & 16.45 & 14.94 & 0.68 & 0.65 \\
\hline S21778 & $\mathrm{O}=\mathrm{c} 1 \mathrm{cnn} 2 \mathrm{c}([\mathrm{nH}] 1) \mathrm{nncc} 2=\mathrm{O}$ & 5.24 & 5.63 & 13.00 & 12.05 & 17.23 & 17.97 & 0.39 & 0.45 \\
\hline $\mathbf{S 2 1 7 9 8}$ & $\mathrm{O}=\mathrm{c} 1 \mathrm{cnnc} 2 \mathrm{n} 1 \mathrm{ncnn} 2$ & 5.64 & 6.69 & 13.23 & 12.72 & 17.03 & 16.98 & 0.40 & 0.43 \\
\hline S218 & $\operatorname{c} 1 \mathrm{cc} 2 \mathrm{c}([\mathrm{nH}] 1)[\mathrm{nH}] \mathrm{cc} 2$ & -7.18 & -6.57 & 3.98 & 5.30 & 18.47 & 18.43 & 0.39 & 0.41 \\
\hline $\mathbf{S 2 1 8 0 6}$ & $\mathrm{O}=\mathrm{c} 1 \mathrm{cnn} 2 \mathrm{c}(\mathrm{s} 1) \mathrm{nncc} 2=\mathrm{O}$ & 5.38 & 6.54 & 12.48 & 11.80 & 15.46 & 15.98 & 0.42 & 0.42 \\
\hline S2189 & $\mathrm{O}=\mathrm{c} 1 \mathrm{oc} 2 \mathrm{cncn} 2 \mathrm{c}(=\mathrm{O})[\mathrm{nH}] 1$ & 3.87 & 3.19 & 10.92 & 9.32 & 14.63 & 14.77 & 0.52 & 0.65 \\
\hline S219 & $\operatorname{c} 1 \mathrm{cc} 2 \mathrm{c}([\mathrm{nH}] 1) \mathrm{cc}[\mathrm{nH}] 2$ & -7.26 & -6.57 & 0.32 & 3.16 & 13.20 & 16.10 & 0.51 & 0.45 \\
\hline S2194 & $\mathrm{O}=\mathrm{c} 1 \mathrm{sc} 2 \mathrm{nccn} 2 \mathrm{c}(=\mathrm{O})[\mathrm{nH}] 1$ & 2.51 & 2.50 & 8.48 & 8.70 & 11.73 & 13.87 & 0.77 & 0.73 \\
\hline S220 & $\mathrm{c} 1 \mathrm{cc} 2 \mathrm{n}(\mathrm{c} 1)[\mathrm{nH}] \mathrm{cc} 2$ & -6.29 & -5.82 & 5.62 & 3.90 & 20.02 & 16.20 & 0.47 & 0.44 \\
\hline S221 & $\mathrm{c} 1 \mathrm{cc} 2 \mathrm{n}(\mathrm{c} 1) \mathrm{cc}[\mathrm{nH}] 2$ & -6.28 & -5.82 & 3.54 & 3.04 & 17.61 & 15.39 & 0.48 & 0.47 \\
\hline $\mathbf{S 2 2 2}$ & {$[\mathrm{nH}] 1 \mathrm{cc} 2 \mathrm{c}(\mathrm{c} 1) \mathrm{cc}[\mathrm{nH}] 2$} & -7.63 & -6.57 & 1.22 & 3.89 & 15.76 & 16.89 & 0.47 & 0.48 \\
\hline $\mathbf{S 2 2 3}$ & $\mathrm{c} 1 \mathrm{oc} 2 \mathrm{c}(\mathrm{c} 1) \mathrm{cc}[\mathrm{nH}] 2$ & -5.43 & -4.97 & 3.08 & 2.80 & 13.33 & 13.66 & 0.56 & 0.50 \\
\hline S224 & $\mathrm{c} 1 \mathrm{cc} 2 \mathrm{c}(\mathrm{o} 1) \mathrm{cc}[\mathrm{nH}] 2$ & -5.43 & -4.97 & 3.48 & 3.55 & 13.63 & 14.18 & 0.37 & 0.34 \\
\hline S2246 & {$[\mathrm{nH}] 1 \mathrm{cc} 2 \mathrm{c}(\mathrm{c} 1) \mathrm{nco} 2$} & -3.92 & -3.14 & 5.02 & 4.79 & 14.45 & 14.68 & 0.61 & 0.56 \\
\hline S2248 & $\operatorname{s1cc} 2 \mathrm{c}(\mathrm{c} 1) \mathrm{nco} 2$ & -2.38 & -2.23 & 2.07 & 2.56 & 7.89 & 10.23 & 0.59 & 0.55 \\
\hline S2249 & $\mathrm{c} 1 \mathrm{nc} 2 \mathrm{c}(\mathrm{o} 1) \mathrm{n}[\mathrm{nH}] \mathrm{c} 2$ & -1.89 & -1.31 & 5.23 & 5.23 & 11.41 & 12.78 & 0.49 & 0.51 \\
\hline $\mathbf{S 2 2 5}$ & $\operatorname{c1cc} 2 n(\mathrm{c} 1) \operatorname{occ} 2$ & -4.77 & -4.22 & 2.37 & 2.46 & 13.01 & 12.53 & 0.44 & 0.48 \\
\hline $\mathbf{S 2 2 5 0}$ & $\mathrm{c} 1 \mathrm{nc} 2 \mathrm{c}(\mathrm{o} 1) \mathrm{c}[\mathrm{nH}] \mathrm{n} 2$ & -1.90 & -1.31 & 5.31 & 6.27 & 14.92 & 15.87 & 0.50 & 0.46 \\
\hline S2251 & c1nc2c(o1)noc2 & 0.59 & 0.29 & 5.15 & 4.67 & 11.54 & 10.22 & 0.44 & 0.53 \\
\hline $\mathbf{S} 2252$ & c1nc2c(o1)con 2 & 0.57 & 0.29 & 6.02 & 5.71 & 14.48 & 13.31 & 0.54 & 0.50 \\
\hline S2256 & c1nc2c(o1)nco2 & 0.51 & 0.29 & 3.23 & 4.38 & 6.61 & 9.98 & 0.33 & 0.36 \\
\hline S2257 & c1oc2c(n1)ncs2 & -0.55 & -0.40 & 3.16 & 4.41 & 10.72 & 12.34 & 0.34 & 0.34 \\
\hline $\mathbf{S} 2258$ & c1nc2c(o1)ncs2 & -0.51 & -0.40 & 2.73 & 3.67 & 6.89 & 9.42 & 0.43 & 0.39 \\
\hline S2259 & $\mathrm{c} 1 \mathrm{nc} 2 \mathrm{c}(\mathrm{o} 1)[\mathrm{nH}] \mathrm{nn} 2$ & 0.70 & 0.52 & 8.73 & 9.47 & 18.42 & 19.71 & 0.47 & 0.46 \\
\hline $\mathbf{S 2 2 6 0}$ & $\mathrm{c} 1 \mathrm{oc} 2 \mathrm{c}(\mathrm{n} 1)[\mathrm{nH}] \mathrm{nn} 2$ & 0.68 & 0.52 & 5.87 & 7.28 & 13.85 & 14.18 & 0.67 & 0.74 \\
\hline S2261 & {$[\mathrm{nH}] 1 \mathrm{nc} 2 \mathrm{c}(\mathrm{n} 1) \mathrm{nco} 2$} & 0.13 & 0.52 & 4.28 & 5.68 & 9.49 & 11.72 & 0.38 & 0.39 \\
\hline
\end{tabular}




\begin{tabular}{|c|c|c|c|c|c|c|c|c|c|}
\hline $\mathbf{S 2 2 6 3}$ & c1oc2c(n1)onn2 & 2.74 & 2.12 & 8.27 & 6.54 & 12.89 & 11.63 & 0.45 & 0.41 \\
\hline S2267 & o1nc2c(n1)nco2 & 2.91 & 2.12 & 9.70 & 6.08 & 14.25 & 10.50 & 0.39 & 0.44 \\
\hline S2268 & s1nc2c(n1)nco2 & 1.66 & 1.43 & 6.38 & 5.24 & 10.30 & 9.61 & 0.67 & 0.55 \\
\hline S227 & $\mathrm{o} 1 \mathrm{cc} 2 \mathrm{c}(\mathrm{c} 1) \mathrm{cc}[\mathrm{nH}] 2$ & -5.41 & -4.97 & 3.52 & 3.30 & 13.70 & 14.00 & 0.43 & 0.39 \\
\hline S2274 & $\operatorname{c} 1 \mathrm{ccc} 2 \mathrm{c}(\mathrm{c} 1) \operatorname{ocn} 2$ & -2.88 & -2.76 & 1.61 & 2.68 & 8.09 & 11.19 & 0.40 & 0.42 \\
\hline S2275 & $\mathrm{c} 1 \mathrm{ncc} 2 \mathrm{c}(\mathrm{c} 1) \mathrm{ocn} 2$ & -0.85 & -0.93 & 4.37 & 4.26 & 10.95 & 11.99 & 0.58 & 0.64 \\
\hline S2276 & $\mathrm{c} 1 \mathrm{ncc} 2 \mathrm{c}(\mathrm{c} 1) \mathrm{nco} 2$ & -0.89 & -0.93 & 4.46 & 3.85 & 10.41 & 10.78 & 0.44 & 0.47 \\
\hline S2277 & $\mathrm{c} 1 \mathrm{cnc} 2 \mathrm{c}(\mathrm{c} 1) \mathrm{nco} 2$ & -1.11 & -0.93 & 2.39 & 3.96 & 8.05 & 10.59 & 0.44 & 0.39 \\
\hline S2278 & $\mathrm{c} 1 \mathrm{cnc} 2 \mathrm{c}(\mathrm{c} 1) \mathrm{ocn} 2$ & -1.08 & -0.93 & 4.06 & 5.08 & 13.51 & 13.91 & 0.46 & 0.49 \\
\hline S2279 & c1nnc2c(c1)ocn2 & 1.07 & 0.90 & 6.80 & 7.68 & 16.99 & 16.96 & 0.75 & 0.66 \\
\hline S228 & $\mathrm{c} 1 \mathrm{sc} 2 \mathrm{c}(\mathrm{c} 1) \mathrm{cc}[\mathrm{nH}] 2$ & -5.92 & -5.66 & 3.31 & 2.15 & 14.02 & 13.21 & 0.50 & 0.47 \\
\hline $\mathbf{S 2 2 8 0}$ & c1nnc2c(c1)nco2 & 1.04 & 0.90 & 5.87 & 6.16 & 13.83 & 12.42 & 0.57 & 0.53 \\
\hline S2281 & $\mathrm{n} 1 \mathrm{ncc} 2 \mathrm{c}(\mathrm{c} 1) \mathrm{ocn} 2$ & 1.33 & 0.90 & 7.83 & 6.45 & 16.64 & 13.83 & 0.33 & 0.35 \\
\hline $\mathbf{S 2 2 8 2}$ & $\mathrm{O}=\mathrm{c} 1[\mathrm{nH}] \mathrm{ccc} 2 \mathrm{c} 1 \mathrm{nco} 2$ & -0.62 & -0.16 & 7.26 & 7.10 & 20.10 & 17.57 & 0.46 & 0.43 \\
\hline $\mathbf{S 2 2 8 3}$ & $\mathrm{O}=\mathrm{c} 1[\mathrm{nH}] \operatorname{ccc} 2 \mathrm{c} 1 \mathrm{ocn} 2$ & -0.48 & -0.16 & 6.48 & 6.33 & 16.18 & 13.87 & 0.39 & 0.41 \\
\hline S2285 & $\mathrm{O}=\mathrm{c} 1 \mathrm{ccc} 2 \mathrm{c}([\mathrm{nH}] 1) \mathrm{nco} 2$ & 0.31 & -0.16 & 6.04 & 5.28 & 16.49 & 13.37 & 0.75 & 0.75 \\
\hline S229 & $\mathrm{c} 1 \mathrm{cc} 2 \mathrm{c}(\mathrm{s} 1) \mathrm{cc}[\mathrm{nH}] 2$ & -5.85 & -5.66 & 3.83 & 2.71 & 14.97 & 13.38 & 0.50 & 0.56 \\
\hline $\mathbf{S 2 3}$ & {$[\mathrm{nH}] 1 \mathrm{cnnc1}$} & -1.37 & -1.57 & 10.04 & 8.62 & 23.00 & 20.45 & 0.47 & 0.48 \\
\hline $\mathbf{S 2 3 0 0}$ & c1ncc $2 \mathrm{c}(\mathrm{n} 1) \mathrm{nco} 2$ & 0.93 & 0.90 & 6.40 & 5.66 & 12.83 & 12.20 & 0.45 & 0.45 \\
\hline $\mathbf{S 2 3 0 1}$ & c1ncc $2 \mathrm{c}(\mathrm{n} 1) \operatorname{ocn} 2$ & 0.93 & 0.90 & 5.31 & 4.95 & 8.65 & 10.09 & 0.42 & 0.42 \\
\hline $\mathbf{S 2 3 0 2}$ & c1nc2c(o1)cnnn2 & 3.19 & 2.73 & 10.49 & 9.28 & 19.35 & 17.50 & 0.67 & 0.67 \\
\hline $\mathbf{S 2 3 0 3}$ & c1nc2c(o1)nnnc2 & 3.18 & 2.73 & 9.47 & 8.17 & 17.22 & 14.17 & 0.63 & 0.56 \\
\hline $\mathbf{S 2 3 0 4}$ & $\mathrm{O}=\mathrm{c} 1[\mathrm{nH}] \mathrm{ncc} 2 \mathrm{c} 1 \mathrm{nco} 2$ & 1.25 & 1.67 & 6.46 & 6.48 & 16.86 & 13.58 & 0.41 & 0.45 \\
\hline $\mathbf{S 2 3 0 5}$ & $\mathrm{O}=\mathrm{c} 1[\mathrm{nH}] \mathrm{ncc} 2 \mathrm{c} 1 \mathrm{ocn} 2$ & 1.45 & 1.67 & 5.69 & 6.11 & 13.31 & 11.08 & 0.47 & 0.58 \\
\hline $\mathbf{S 2 3 0 8}$ & $\mathrm{O}=\mathrm{c} 1 \mathrm{ncc} 2 \mathrm{c}([\mathrm{nH}] 1) \mathrm{nco} 2$ & 2.20 & 1.67 & 9.44 & 8.23 & 21.57 & 17.13 & 0.73 & 0.79 \\
\hline S231 & $\operatorname{c} 1 \mathrm{cc} 2 n(\mathrm{c} 1) \operatorname{ccs} 2$ & -4.93 & -4.91 & 2.47 & 1.96 & 12.78 & 12.01 & 0.38 & 0.46 \\
\hline S232 & $\operatorname{s1cc} 2 c(c 1) c c[n H] 2$ & -5.84 & -5.66 & 3.51 & 2.52 & 14.38 & 13.32 & 0.71 & 0.65 \\
\hline $\mathbf{S 2 3 2 9}$ & $\mathrm{O}=\mathrm{c} 1 \mathrm{cnc} 2 \mathrm{c}([\mathrm{nH}] 1) \mathrm{nco} 2$ & 2.04 & 1.67 & 6.50 & 7.06 & 13.78 & 12.72 & 0.43 & 0.45 \\
\hline $\mathbf{S 2 3 3}$ & $\mathrm{c} 1 \mathrm{cc} 2 \mathrm{c}([\mathrm{nH}] 1)[\mathrm{nH}] \mathrm{nc} 2$ & -5.04 & -4.74 & 6.37 & 6.48 & 17.67 & 18.40 & 0.60 & 0.58 \\
\hline $\mathbf{S 2 3 3 1}$ & $\mathrm{O}=\mathrm{c} 1 \mathrm{nnn} 2 \mathrm{c}([\mathrm{nH}] 1) \mathrm{nco} 2$ & 3.98 & 3.50 & 11.08 & 11.03 & 20.91 & 18.73 & 0.40 & 0.35 \\
\hline $\mathbf{S 2 3 3 3}$ & $\mathrm{O}=\mathrm{c} 1[\mathrm{nH}] \mathrm{c}(=\mathrm{O}) \mathrm{c} 2 \mathrm{c}([\mathrm{nH}] 1) \mathrm{nco} 2$ & 2.70 & 2.44 & 7.44 & 7.47 & 13.70 & 12.70 & 0.55 & 0.50 \\
\hline
\end{tabular}




\begin{tabular}{|c|c|c|c|c|c|c|c|c|c|}
\hline S234 & $\mathrm{c} 1[\mathrm{nH}] \mathrm{c} 2 \mathrm{c}(\mathrm{c} 1)[\mathrm{nH}] \mathrm{nc} 2$ & -5.13 & -4.74 & 3.58 & 4.35 & 14.63 & 16.08 & 0.80 & 0.73 \\
\hline $\mathbf{S 2 3 4 0}$ & $\mathrm{O}=\mathrm{c} 1 \mathrm{sc}(=\mathrm{O}) \mathrm{c} 2 \mathrm{c}([\mathrm{nH}] 1) \mathrm{nco} 2$ & 3.03 & 3.35 & 9.05 & 9.19 & 14.75 & 14.06 & 0.70 & 0.68 \\
\hline S2343 & $\mathrm{O}=\mathrm{c} 1 \mathrm{cc}[\mathrm{nH}] \mathrm{c} 2 \mathrm{c} 1 \mathrm{ocn} 2$ & -0.56 & -0.16 & 8.74 & 7.46 & 21.95 & 16.36 & 0.57 & 0.51 \\
\hline S2344 & $\mathrm{O}=\mathrm{c} 1 \mathrm{cc}[\mathrm{nH}] \mathrm{c} 2 \mathrm{c} 1 \mathrm{nco} 2$ & -0.67 & -0.16 & 11.59 & 9.94 & 27.30 & 23.64 & 0.46 & 0.42 \\
\hline S2345 & $\mathrm{O}=\mathrm{c} 1 \mathrm{nc}[\mathrm{nH}] \mathrm{c} 2 \mathrm{c} 1 \mathrm{ocn} 2$ & 1.35 & 1.67 & 10.83 & 10.42 & 24.36 & 20.12 & 0.55 & 0.54 \\
\hline S2346 & $\mathrm{O}=\mathrm{c} 1 \mathrm{nc}[\mathrm{nH}] \mathrm{c} 2 \mathrm{c} 1 \mathrm{nco} 2$ & 1.21 & 1.67 & 13.80 & 13.30 & 29.28 & 28.61 & 0.51 & 0.50 \\
\hline S2347 & $\mathrm{c} 1 \mathrm{cnc} 2 \mathrm{c}(\mathrm{n} 1) \mathrm{ocn} 2$ & 0.69 & 0.90 & 4.41 & 5.34 & 9.92 & 11.06 & 0.50 & 0.55 \\
\hline S2348 & c1nnc2c(n1)nco2 & 2.87 & 2.73 & 8.66 & 6.94 & 13.26 & 11.60 & 0.75 & 0.70 \\
\hline S2349 & c1nnc2c(n1)ocn2 & 2.88 & 2.73 & 8.54 & 7.35 & 14.78 & 12.82 & 0.42 & 0.47 \\
\hline S235 & $\mathrm{c} 1 \mathrm{cn} 2 \mathrm{c}(\mathrm{c} 1) \mathrm{cn}[\mathrm{nH}] 2$ & -4.27 & -3.99 & 4.89 & 5.28 & 14.60 & 16.39 & 0.43 & 0.45 \\
\hline $\mathbf{S 2 3 5 0}$ & $\mathrm{O}=\mathrm{c} 1[\mathrm{nH}] \mathrm{cnc} 2 \mathrm{c} 1 \mathrm{ocn} 2$ & 1.27 & 1.67 & 8.46 & 8.26 & 14.99 & 14.47 & 0.43 & 0.48 \\
\hline S2351 & $\mathrm{O}=\mathrm{c} 1[\mathrm{nH}] \mathrm{cnc} 2 \mathrm{c} 1 \mathrm{nco} 2$ & 1.08 & 1.67 & 9.06 & 7.92 & 18.59 & 14.85 & 0.52 & 0.58 \\
\hline S236 & $\mathrm{c} 1 \mathrm{cn} 2 \mathrm{c}(\mathrm{c} 1)[\mathrm{nH}] \mathrm{nc} 2$ & -4.19 & -3.99 & 2.22 & 4.43 & 11.75 & 15.58 & 0.54 & 0.54 \\
\hline $\mathbf{S 2 3 6 0}$ & $\mathrm{n} 1 \mathrm{nnc} 2 \mathrm{c}(\mathrm{n} 1) \mathrm{ocn} 2$ & 5.12 & 4.56 & 13.10 & 9.98 & 18.89 & 15.60 & 0.67 & 0.62 \\
\hline S2367 & $\mathrm{O}=\mathrm{c} 1[\mathrm{nH}] \mathrm{nnc} 2 \mathrm{c} 1 \mathrm{nco} 2$ & 2.96 & 3.50 & 7.14 & 8.32 & 13.82 & 13.09 & 0.51 & 0.51 \\
\hline $\mathbf{S 2 3 7}$ & $\mathrm{c} 1 \mathrm{cc} 2 \mathrm{c}([\mathrm{nH}] 1) \mathrm{n}[\mathrm{nH}] \mathrm{c} 2$ & -5.78 & -4.74 & 1.25 & 3.81 & 11.68 & 14.91 & 0.39 & 0.34 \\
\hline S23721 & $\mathrm{O}=\mathrm{c} 1 \mathrm{sc}(=\mathrm{O}) \mathrm{n} 2 \mathrm{c}(\mathrm{n} 1) \mathrm{scc} 2$ & 2.80 & 3.41 & 9.76 & 9.84 & 18.98 & 16.05 & 0.48 & 0.49 \\
\hline $\mathbf{S} 238$ & $\mathrm{c} 1 \mathrm{cc} 2 \mathrm{c}([\mathrm{nH}] 1) \mathrm{c}[\mathrm{nH}] \mathrm{n} 2$ & -5.79 & -4.74 & 3.57 & 5.72 & 16.58 & 17.86 & 0.37 & 0.37 \\
\hline S2384 & $\mathrm{O}=\mathrm{c} 1 \mathrm{cn}[\mathrm{nH}] \mathrm{c} 2 \mathrm{c} 1 \mathrm{nco} 2$ & 1.18 & 1.67 & 10.25 & 9.32 & 22.49 & 19.64 & 0.53 & 0.47 \\
\hline S23868 & $\mathrm{O}=\mathrm{c} 1 \mathrm{oc} 2 \mathrm{c}(\mathrm{o} 1) \mathrm{csc} 2$ & 0.85 & 0.14 & 4.10 & 5.33 & 11.47 & 13.28 & 0.44 & 0.48 \\
\hline S239 & c1cc2c([nH]1)onc2 & -3.04 & -3.14 & 5.72 & 4.94 & 15.77 & 14.98 & 0.47 & 0.47 \\
\hline $\mathbf{S 2 3 9 2}$ & $\mathrm{O}=\mathrm{c} 1 \mathrm{nn}[\mathrm{nH}] \mathrm{c} 2 \mathrm{c} 1 \mathrm{nco} 2$ & 2.95 & 3.50 & 12.99 & 13.70 & 25.34 & 26.86 & 0.41 & 0.44 \\
\hline $\mathbf{S 2 3 9 5 0}$ & $\mathrm{O}=\mathrm{c} 1 \mathrm{oc} 2 \mathrm{cscc} 2 \mathrm{oc} 1=\mathrm{O}$ & 2.80 & 3.12 & 8.02 & 9.35 & 14.63 & 20.50 & 0.32 & 0.33 \\
\hline S24 & $\operatorname{clncn}[\mathrm{nH}] 1$ & -1.71 & -1.57 & 6.75 & 5.11 & 13.81 & 13.95 & 0.41 & 0.36 \\
\hline S240 & $\mathrm{c} 1 \mathrm{cc} 2 \mathrm{c}([\mathrm{nH}] 1) \mathrm{cno} 2$ & -3.03 & -3.14 & 7.29 & 5.69 & 18.47 & 15.49 & 0.50 & 0.45 \\
\hline S24119 & $\mathrm{O}=\mathrm{c} 1 \mathrm{sc} 2 \mathrm{c}(\mathrm{s} 1) \operatorname{con} 2$ & 1.46 & 1.28 & 7.07 & 6.06 & 11.02 & 12.17 & 0.70 & 0.66 \\
\hline S24120 & $\mathrm{O}=\mathrm{c} 1 \mathrm{sc} 2 \mathrm{c}(\mathrm{s} 1) \operatorname{csn} 2$ & 0.63 & 0.59 & 6.71 & 5.33 & 12.28 & 11.62 & 0.44 & 0.44 \\
\hline S243 & c1cc2c([nH]1)noc2 & -3.22 & -3.14 & 4.15 & 4.17 & 14.34 & 13.36 & 0.38 & 0.39 \\
\hline S244 & $\operatorname{c} 1 \mathrm{cc} 2 \mathrm{c}([\mathrm{nH}] 1) \operatorname{con} 2$ & -3.22 & -3.14 & 7.37 & 6.08 & 19.70 & 16.31 & 0.45 & 0.49 \\
\hline S245 & $\mathrm{c} 1 \mathrm{cc} 2 \mathrm{c}([\mathrm{nH}] 1) \mathrm{snc} 2$ & -3.77 & -3.83 & 6.10 & 4.23 & 16.25 & 14.36 & 0.69 & 0.59 \\
\hline S246 & $\mathrm{c} 1 \mathrm{cc} 2 \mathrm{c}([\mathrm{nH}] 1) \mathrm{cns} 2$ & -3.70 & -3.83 & 6.65 & 4.79 & 17.07 & 14.53 & 0.57 & 0.59 \\
\hline
\end{tabular}




\begin{tabular}{|c|c|c|c|c|c|c|c|c|c|}
\hline S248 & $\mathrm{c} 1 \mathrm{cn} 2 \mathrm{c}(\mathrm{c} 1) \mathrm{snc} 2$ & -2.85 & -3.08 & 3.76 & 4.24 & 9.94 & 13.37 & 0.43 & 0.39 \\
\hline S249 & $\mathrm{c} 1 \mathrm{cc} 2 \mathrm{c}([\mathrm{nH}] 1) \mathrm{nsc} 2$ & -4.06 & -3.83 & 3.08 & 3.33 & 11.92 & 12.52 & 0.66 & 0.71 \\
\hline S2499 & {$[\mathrm{nH}] 1 \mathrm{cc} 2 \mathrm{c}(\mathrm{c} 1) \mathrm{ncs} 2$} & -4.72 & -3.83 & 5.36 & 4.11 & 15.26 & 14.27 & 0.47 & 0.50 \\
\hline S2500 & o1cc2c(c1)ncs2 & -2.56 & -2.23 & 1.34 & 2.64 & 7.18 & 10.43 & 0.54 & 0.49 \\
\hline S2501 & $\operatorname{s} 1 \mathrm{cc} 2 \mathrm{c}(\mathrm{c} 1) \mathrm{ncs} 2$ & -3.10 & -2.92 & 1.21 & 1.92 & 7.56 & 9.87 & 0.55 & 0.48 \\
\hline S2502 & $\mathrm{c} 1 \mathrm{nc} 2 \mathrm{c}(\mathrm{s} 1) \mathrm{n}[\mathrm{nH}] \mathrm{c} 2$ & -2.91 & -2.00 & 5.25 & 4.47 & 11.55 & 12.13 & 0.55 & 0.58 \\
\hline S2503 & $\mathrm{c} 1 \mathrm{nc} 2 \mathrm{c}(\mathrm{s} 1) \mathrm{c}[\mathrm{nH}] \mathrm{n} 2$ & -2.79 & -2.00 & 5.40 & 5.67 & 15.78 & 15.67 & 0.42 & 0.43 \\
\hline S2504 & c1nc2c(s1)noc2 & -0.43 & -0.40 & 4.44 & 3.95 & 10.89 & 9.64 & 0.66 & 0.61 \\
\hline S2505 & $\mathrm{c} 1 \mathrm{nc} 2 \mathrm{c}(\mathrm{s} 1) \operatorname{con} 2$ & -0.28 & -0.40 & 5.06 & 5.15 & 14.05 & 13.17 & 0.34 & 0.37 \\
\hline S2506 & $\mathrm{c} 1 \mathrm{nc} 2 \mathrm{c}(\mathrm{s} 1) \mathrm{nsc} 2$ & -1.35 & -1.09 & 2.78 & 3.16 & 7.95 & 8.91 & 0.74 & 0.72 \\
\hline S2507 & $\mathrm{c} 1 \mathrm{nc} 2 \mathrm{c}(\mathrm{s} 1) \operatorname{csn} 2$ & -1.20 & -1.09 & 4.63 & 4.37 & 13.38 & 12.44 & 0.53 & 0.57 \\
\hline S2508 & $\mathrm{c} 1 \mathrm{nc} 2 \mathrm{c}(\mathrm{s} 1) \operatorname{sen} 2$ & -1.40 & -1.09 & 2.35 & 3.81 & 10.76 & 12.17 & 0.45 & 0.45 \\
\hline S2509 & $\mathrm{c} 1 \mathrm{nc} 2 \mathrm{c}(\mathrm{s} 1) \mathrm{ncs} 2$ & -1.43 & -1.09 & 2.21 & 2.96 & 6.41 & 8.84 & 0.54 & 0.47 \\
\hline $\mathbf{S} 251$ & $\mathrm{c} 1[\mathrm{nH}] \mathrm{c} 2 \mathrm{c}(\mathrm{c} 1) \mathrm{nc}[\mathrm{nH}] 2$ & -5.30 & -4.74 & 6.94 & 8.98 & 21.39 & 22.27 & 0.54 & 0.57 \\
\hline $\mathbf{S 2 5 1 0}$ & $\mathrm{c} 1 \mathrm{nc} 2 \mathrm{c}(\mathrm{s} 1)[\mathrm{nH}] \mathrm{nn} 2$ & -0.16 & -0.17 & 8.31 & 9.02 & 18.68 & 19.80 & 0.59 & 0.59 \\
\hline S2511 & $\mathrm{c} 1 \mathrm{sc} 2 \mathrm{c}(\mathrm{n} 1)[\mathrm{nH}] \mathrm{nn} 2$ & -0.25 & -0.17 & 4.88 & 6.47 & 13.10 & 13.48 & 0.75 & 0.83 \\
\hline S2512 & {$[\mathrm{nH}] 1 \mathrm{nc} 2 \mathrm{c}(\mathrm{n} 1) \mathrm{ncs} 2$} & -0.95 & -0.17 & 4.13 & 5.00 & 10.43 & 11.29 & 0.41 & 0.42 \\
\hline $\mathbf{S 2 5 1 3}$ & c1nc2c(s1)onn2 & 1.60 & 1.43 & 6.95 & 6.59 & 11.94 & 14.06 & 0.56 & 0.59 \\
\hline S2514 & c1nc2c(s1)nno2 & 1.79 & 1.43 & 7.40 & 5.86 & 12.83 & 11.14 & 0.50 & 0.46 \\
\hline S2515 & $\mathrm{c} 1 \mathrm{nc} 2 \mathrm{c}(\mathrm{s} 1) \mathrm{snn} 2$ & 0.79 & 0.74 & 6.20 & 5.93 & 13.82 & 13.73 & 0.46 & 0.50 \\
\hline S2516 & $\mathrm{c} 1 \mathrm{sc} 2 \mathrm{c}(\mathrm{n} 1) \operatorname{snn} 2$ & 0.75 & 0.74 & 6.10 & 5.08 & 12.18 & 10.39 & 0.68 & 0.66 \\
\hline S2518 & o1nc2c(n1)ncs2 & 1.87 & 1.43 & 8.38 & 5.44 & 13.75 & 10.13 & 0.42 & 0.44 \\
\hline S2519 & $\mathrm{s} 1 \mathrm{nc} 2 \mathrm{c}(\mathrm{n} 1) \mathrm{ncs} 2$ & 0.60 & 0.74 & 5.24 & 4.59 & 10.10 & 9.24 & 0.50 & 0.50 \\
\hline $\mathbf{S 2 5 2}$ & $\mathrm{c} 1[\mathrm{nH}] \mathrm{c} 2 \mathrm{c}(\mathrm{c} 1)[\mathrm{nH}] \mathrm{cn} 2$ & -5.42 & -4.74 & 3.39 & 4.94 & 14.33 & 16.99 & 0.85 & 0.79 \\
\hline $\mathbf{S 2 5 2 0}$ & $\mathrm{O}=\mathrm{c} 1[\mathrm{nH}] \mathrm{c} 2 \mathrm{c}([\mathrm{nH}] 1) \mathrm{ncs} 2$ & -0.57 & -1.23 & 4.77 & 5.26 & 13.92 & 14.53 & 0.45 & 0.45 \\
\hline $\mathbf{S} 2522$ & $\mathrm{O}=\mathrm{c} 1 \mathrm{oc} 2 \mathrm{c}([\mathrm{nH}] 1) \operatorname{sen} 2$ & 1.09 & 0.37 & 8.32 & 8.01 & 17.15 & 16.59 & 0.55 & 0.56 \\
\hline S2525 & $\operatorname{c} 1 \operatorname{ccc} 2 \mathrm{c}(\mathrm{c} 1) \operatorname{sen} 2$ & -3.50 & -3.45 & 0.74 & 2.03 & 7.71 & 10.82 & 0.40 & 0.44 \\
\hline S2526 & $\mathrm{c} 1 \mathrm{ncc} 2 \mathrm{c}(\mathrm{c} 1) \operatorname{sen} 2$ & -1.47 & -1.62 & 3.57 & 3.66 & 10.54 & 11.76 & 0.32 & 0.33 \\
\hline $\mathbf{S 2 5 2 7}$ & $\mathrm{c} 1 \mathrm{ncc} 2 \mathrm{c}(\mathrm{c} 1) \mathrm{ncs} 2$ & -1.53 & -1.62 & 3.71 & 3.19 & 9.87 & 10.37 & 0.73 & 0.77 \\
\hline S2528 & $\mathrm{c} 1 \mathrm{cnc} 2 \mathrm{c}(\mathrm{c} 1) \mathrm{ncs} 2$ & -2.01 & -1.62 & 2.08 & 3.21 & 7.55 & 9.94 & 0.37 & 0.44 \\
\hline S2529 & $\mathrm{c} 1 \mathrm{cnc} 2 \mathrm{c}(\mathrm{c} 1) \operatorname{sen} 2$ & -1.83 & -1.62 & 3.97 & 4.50 & 14.01 & 13.74 & 0.64 & 0.58 \\
\hline
\end{tabular}




\begin{tabular}{|c|c|c|c|c|c|c|c|c|c|}
\hline $\mathbf{S 2 5 3}$ & $\mathrm{c} 1 \mathrm{cn} 2 \mathrm{c}(\mathrm{c} 1) \mathrm{nc}[\mathrm{nH}] 2$ & -4.54 & -3.99 & 8.84 & 7.13 & 21.39 & 19.26 & 0.64 & 0.58 \\
\hline S2530 & $\mathrm{c} 1 \mathrm{nnn} 2 \mathrm{c}(\mathrm{c} 1) \operatorname{scn} 2$ & 0.31 & 0.21 & 6.71 & 7.15 & 17.50 & 16.92 & 0.62 & 0.67 \\
\hline S2531 & $\mathrm{c} 1 \mathrm{nnc} 2 \mathrm{c}(\mathrm{c} 1) \mathrm{ncs} 2$ & 0.08 & 0.21 & 5.23 & 5.39 & 13.37 & 11.74 & 0.37 & 0.37 \\
\hline S2532 & $\mathrm{n} 1 \mathrm{ncc} 2 \mathrm{c}(\mathrm{c} 1) \operatorname{sen} 2$ & 0.70 & 0.21 & 7.07 & 5.84 & 16.51 & 13.55 & 0.64 & 0.58 \\
\hline $\mathbf{S 2 5 3 3}$ & $\mathrm{O}=\mathrm{c} 1[\mathrm{nH}] \mathrm{ccc} 2 \mathrm{c} 1 \mathrm{ncs} 2$ & -1.43 & -0.85 & 6.68 & 6.47 & 20.04 & 17.38 & 0.76 & 0.73 \\
\hline $\mathbf{S 2 5 3 4}$ & $\mathrm{O}=\mathrm{c} 1[\mathrm{nH}] \operatorname{ccc} 2 \mathrm{c} 1 \mathrm{scn} 2$ & -1.36 & -0.85 & 6.00 & 5.58 & 15.29 & 13.15 & 0.52 & 0.56 \\
\hline S2535 & $\mathrm{O}=\mathrm{c} 1 \mathrm{ccc} 2 \mathrm{c}([\mathrm{nH}] 1) \operatorname{sen} 2$ & -0.18 & -0.85 & 5.09 & 5.53 & 14.53 & 14.09 & 0.58 & 0.54 \\
\hline S2536 & $\mathrm{O}=\mathrm{c} 1 \mathrm{ccc} 2 \mathrm{c}([\mathrm{nH}] 1) \mathrm{ncs} 2$ & -0.24 & -0.85 & 5.30 & 4.60 & 16.08 & 13.03 & 0.54 & 0.53 \\
\hline S254 & $\mathrm{c} 1 \mathrm{cn} 2 \mathrm{c}(\mathrm{c} 1)[\mathrm{nH}] \mathrm{cn} 2$ & -4.75 & -3.99 & 7.15 & 5.51 & 19.01 & 17.42 & 0.61 & 0.74 \\
\hline S2540 & $\mathrm{O}=\mathrm{c} 1 \mathrm{occc} 2 \mathrm{c} 1 \mathrm{scn} 2$ & 0.30 & 0.75 & 4.70 & 5.57 & 13.36 & 11.83 & 0.39 & 0.42 \\
\hline $\mathbf{S 2 5 5}$ & $\mathrm{c} 1 \mathrm{nc} 2 \mathrm{c}(\mathrm{o} 1)[\mathrm{nH}] \mathrm{cc} 2$ & -3.57 & -3.14 & 5.60 & 4.94 & 15.46 & 15.34 & 0.57 & 0.55 \\
\hline S2551 & c1ncc $2 \mathrm{c}(\mathrm{n} 1) \mathrm{ncs} 2$ & 0.18 & 0.21 & 5.24 & 5.07 & 12.42 & 11.99 & 0.54 & 0.50 \\
\hline S2552 & $\mathrm{c} 1 \mathrm{ncc} 2 \mathrm{c}(\mathrm{n} 1) \operatorname{scn} 2$ & 0.05 & 0.21 & 4.76 & 4.25 & 8.73 & 9.58 & 0.59 & 0.62 \\
\hline $\mathbf{S 2 5 5 3}$ & $\mathrm{c} 1 \mathrm{nc} 2 \mathrm{c}(\mathrm{s} 1) \mathrm{cnnn} 2$ & 2.47 & 2.04 & 9.12 & 8.74 & 18.70 & 17.42 & 0.43 & 0.47 \\
\hline S2554 & $\mathrm{c} 1 \mathrm{nc} 2 \mathrm{c}(\mathrm{s} 1) \mathrm{nnn} \mathrm{c} 2$ & 2.27 & 2.04 & 8.60 & 7.45 & 17.01 & 13.62 & 0.82 & 0.82 \\
\hline S2555 & $\mathrm{O}=\mathrm{c} 1[\mathrm{nH}] \mathrm{ncc} 2 \mathrm{c} 1 \mathrm{ncs} 2$ & 0.48 & 0.98 & 5.90 & 5.84 & 17.05 & 13.34 & 0.36 & 0.38 \\
\hline S2556 & $\mathrm{O}=\mathrm{c} 1[\mathrm{nH}] \mathrm{ncc} 2 \mathrm{c} 1 \mathrm{scn} 2$ & 0.61 & 0.98 & 5.39 & 5.41 & 12.78 & 10.49 & 0.43 & 0.45 \\
\hline S256 & $\mathrm{c} 1 \mathrm{oc} 2 \mathrm{c}(\mathrm{n} 1)[\mathrm{nH}] \mathrm{cc} 2$ & -3.59 & -3.14 & 3.21 & 3.78 & 9.89 & 12.90 & 0.56 & 0.58 \\
\hline S2560 & $\mathrm{O}=\mathrm{c} 1 \mathrm{ncc} 2 \mathrm{c}([\mathrm{nH}] 1) \operatorname{sen} 2$ & 1.77 & 0.98 & 8.31 & 8.94 & 19.81 & 19.19 & 0.40 & 0.43 \\
\hline $\mathbf{S} 257$ & $\mathrm{c} 1 \mathrm{cn} 2 \mathrm{c}(\mathrm{c} 1) \mathrm{nco} 2$ & -2.99 & -2.39 & 4.05 & 4.15 & 12.33 & 13.43 & 0.53 & 0.47 \\
\hline S2579 & $\mathrm{O}=\mathrm{c} 1 \mathrm{cnc} 2 \mathrm{c}([\mathrm{nH}] 1) \operatorname{sen} 2$ & 1.41 & 0.98 & 8.20 & 8.50 & 14.81 & 16.96 & 0.42 & 0.46 \\
\hline S258 & $\mathrm{c} 1 \mathrm{cn} 2 \mathrm{c}(\mathrm{c} 1) \operatorname{ocn} 2$ & -2.99 & -2.39 & 4.00 & 3.68 & 12.03 & 12.58 & 0.51 & 0.49 \\
\hline S2581 & $\mathrm{O}=\mathrm{c} 1 \mathrm{nnn} 2 \mathrm{c}([\mathrm{nH}] 1) \operatorname{sen} 2$ & 3.37 & 2.81 & 11.86 & 12.93 & 21.29 & 24.31 & 0.66 & 0.69 \\
\hline $\mathbf{S 2 5 8 3}$ & $\mathrm{O}=\mathrm{c} 1[\mathrm{nH}] \mathrm{c}(=\mathrm{O}) \mathrm{c} 2 \mathrm{c}([\mathrm{nH}] 1) \mathrm{scn} 2$ & 1.90 & 1.75 & 9.37 & 8.52 & 18.87 & 17.29 & 0.67 & 0.74 \\
\hline S2585 & $\mathrm{O}=\mathrm{c} 1[\mathrm{nH}] \mathrm{c} 2 \mathrm{scnc} 2[\mathrm{nH}] \mathrm{c} 1=\mathrm{O}$ & 2.00 & 1.75 & 8.21 & 9.50 & 19.21 & 22.18 & 0.45 & 0.49 \\
\hline S2586 & $\mathrm{O}=\mathrm{c} 1 \mathrm{oc}(=\mathrm{O}) \mathrm{c} 2 \mathrm{c}([\mathrm{nH}] 1) \operatorname{sen} 2$ & 3.37 & 3.35 & 11.93 & 11.92 & 21.19 & 21.32 & 0.79 & 0.70 \\
\hline S2588 & $\mathrm{O}=\mathrm{c} 1[\mathrm{nH}] \mathrm{c} 2 \mathrm{scnc} 2 \mathrm{oc} 1=\mathrm{O}$ & 3.22 & 3.35 & 10.77 & 12.23 & 19.83 & 24.05 & 0.51 & 0.49 \\
\hline $\mathbf{S 2 5 9}$ & $\mathrm{c} 1 \mathrm{nc} 2 \mathrm{c}(\mathrm{s} 1)[\mathrm{nH}] \mathrm{cc} 2$ & -4.31 & -3.83 & 5.40 & 4.38 & 15.70 & 15.15 & 0.57 & 0.56 \\
\hline $\mathbf{S 2 5 9 0}$ & $\mathrm{O}=\mathrm{c} 1 \mathrm{sc}(=\mathrm{O}) \mathrm{c} 2 \mathrm{c}([\mathrm{nH}] 1) \operatorname{sen} 2$ & 2.32 & 2.66 & 10.54 & 10.89 & 18.91 & 19.79 & 0.58 & 0.66 \\
\hline S2594 & $\mathrm{O}=\mathrm{c} 1 \mathrm{cc}[\mathrm{nH}] \mathrm{c} 2 \mathrm{c} 1 \mathrm{scn} 2$ & -1.34 & -0.85 & 7.80 & 6.62 & 20.53 & 15.45 & 0.46 & 0.56 \\
\hline S2595 & $\mathrm{O}=\mathrm{c} 1 \mathrm{cc}[\mathrm{nH}] \mathrm{c} 2 \mathrm{c} 1 \mathrm{ncs} 2$ & -1.41 & -0.85 & 11.19 & 9.49 & 27.38 & 23.77 & 0.57 & 0.52 \\
\hline
\end{tabular}




\begin{tabular}{|c|c|c|c|c|c|c|c|c|c|}
\hline S2596 & $\mathrm{O}=\mathrm{c} 1 \mathrm{nc}[\mathrm{nH}] \mathrm{c} 2 \mathrm{c} 1 \mathrm{scn} 2$ & 0.56 & 0.98 & 9.74 & 9.56 & 23.10 & 19.17 & 0.54 & 0.47 \\
\hline S2597 & $\mathrm{O}=\mathrm{c} 1 \mathrm{nc}[\mathrm{nH}] \mathrm{c} 2 \mathrm{c} 1 \mathrm{ncs} 2$ & 0.49 & 0.98 & 13.33 & 12.90 & 29.39 & 28.87 & 0.50 & 0.46 \\
\hline S2598 & $\mathrm{c} 1 \mathrm{cnc} 2 \mathrm{c}(\mathrm{n} 1) \operatorname{scn} 2$ & -0.35 & 0.21 & 3.44 & 4.66 & 10.06 & 10.62 & 0.36 & 0.37 \\
\hline S2599 & c1nnc2c(n1)ncs2 & 1.81 & 2.04 & 7.38 & 6.25 & 12.36 & 11.11 & 0.51 & 0.45 \\
\hline $\mathbf{S 2 6 0}$ & $\mathrm{c} 1 \mathrm{sc} 2 \mathrm{c}(\mathrm{n} 1)[\mathrm{nH}] \mathrm{cc} 2$ & -4.29 & -3.83 & 2.81 & 3.04 & 10.31 & 12.37 & 0.40 & 0.42 \\
\hline S2600 & $\mathrm{c} 1 \mathrm{nnc} 2 \mathrm{c}(\mathrm{n} 1) \operatorname{sen} 2$ & 1.85 & 2.04 & 7.26 & 6.72 & 14.41 & 12.50 & 0.51 & 0.57 \\
\hline S2601 & $\mathrm{O}=\mathrm{c} 1[\mathrm{nH}] \mathrm{cnc} 2 \mathrm{c} 1 \mathrm{scn} 2$ & 0.27 & 0.98 & 7.85 & 7.59 & 14.24 & 13.94 & 0.67 & 0.61 \\
\hline $\mathrm{S} 2602$ & $\mathrm{O}=\mathrm{c} 1[\mathrm{nH}] \mathrm{cnc} 2 \mathrm{c} 1 \mathrm{ncs} 2$ & 0.02 & 0.98 & 8.23 & 7.19 & 18.59 & 14.37 & 0.35 & 0.42 \\
\hline S2605 & $\mathrm{O}=\mathrm{c} 1 \mathrm{ocnc} 2 \mathrm{c} 1 \mathrm{scn} 2$ & 1.95 & 2.58 & 5.85 & 6.69 & 11.59 & 11.37 & 0.65 & 0.67 \\
\hline S2611 & $\mathrm{n} 1 \mathrm{nnc} 2 \mathrm{c}(\mathrm{n} 1) \operatorname{scn} 2$ & 4.11 & 3.87 & 11.43 & 9.33 & 18.24 & 15.25 & 0.63 & 0.56 \\
\hline S2618 & $\mathrm{O}=\mathrm{c} 1[\mathrm{nH}] \mathrm{nnc} 2 \mathrm{c} 1 \mathrm{ncs} 2$ & 1.90 & 2.81 & 6.51 & 7.57 & 14.31 & 12.58 & 0.78 & 0.78 \\
\hline S2619 & $\mathrm{O}=\mathrm{c} 1[\mathrm{nH}] \mathrm{nnc} 2 \mathrm{c} 1 \mathrm{scn} 2$ & 2.21 & 2.81 & 6.73 & 8.45 & 11.72 & 13.53 & 0.49 & 0.43 \\
\hline $\mathbf{S 2 6 2}$ & $\operatorname{c} 1 \mathrm{cn} 2 \mathrm{c}(\mathrm{c} 1) \operatorname{sen} 2$ & -3.66 & -3.08 & 2.87 & 2.99 & 11.20 & 12.14 & 0.51 & 0.45 \\
\hline S2628 & $\mathrm{O}=\mathrm{c} 1 \mathrm{ccoc} 2 \mathrm{c} 1 \mathrm{scn} 2$ & 0.19 & 0.75 & 4.66 & 5.21 & 13.33 & 11.32 & 0.57 & 0.60 \\
\hline S263 & $\operatorname{c1cc} 2 \mathrm{c}([\mathrm{nH}] 1)[\mathrm{nH}] \mathrm{nn} 2$ & -3.10 & -2.91 & 9.12 & 10.80 & 22.69 & 23.63 & 0.58 & 0.54 \\
\hline S2630 & $\mathrm{O}=\mathrm{c} 1 \mathrm{n} \operatorname{coc} 2 \mathrm{c} 1 \mathrm{scn} 2$ & 2.05 & 2.58 & 6.72 & 7.26 & 16.03 & 13.79 & 0.54 & 0.49 \\
\hline S2634 & $\mathrm{O}=\mathrm{c} 1 \mathrm{cn}[\mathrm{nH}] \mathrm{c} 2 \mathrm{c} 1 \mathrm{scn} 2$ & 0.54 & 0.98 & 5.86 & 6.45 & 15.48 & 12.80 & 0.51 & 0.48 \\
\hline S2635 & $\mathrm{O}=\mathrm{c} 1 \mathrm{cn}[\mathrm{nH}] \mathrm{c} 2 \mathrm{c} 1 \mathrm{ncs} 2$ & 0.41 & 0.98 & 9.84 & 8.85 & 22.66 & 19.73 & 0.47 & 0.43 \\
\hline S264 & $\mathrm{c} 1 \mathrm{cc} 2 \mathrm{c}([\mathrm{nH}] 1) \mathrm{nn}[\mathrm{nH}] 2$ & -3.19 & -2.91 & 3.66 & 6.75 & 15.45 & 18.36 & 0.44 & 0.54 \\
\hline S2642 & $\mathrm{O}=\mathrm{c} 1 \mathrm{nn}[\mathrm{nH}] \mathrm{c} 2 \mathrm{c} 1 \mathrm{scn} 2$ & 2.40 & 2.81 & 8.56 & 10.41 & 19.47 & 18.76 & 0.47 & 0.51 \\
\hline S2643 & $\mathrm{O}=\mathrm{c} 1 \mathrm{nn}[\mathrm{nH}] \mathrm{c} 2 \mathrm{c} 1 \mathrm{ncs} 2$ & 2.25 & 2.81 & 12.55 & 13.28 & 25.65 & 27.08 & 0.40 & 0.42 \\
\hline S265 & $\mathrm{c} 1 \mathrm{cn} 2 \mathrm{c}(\mathrm{c} 1) \mathrm{nn}[\mathrm{nH}] 2$ & -2.44 & -2.16 & 8.51 & 9.15 & 17.54 & 20.84 & 0.32 & 0.32 \\
\hline S266 & $\mathrm{c} 1 \mathrm{cn} 2 \mathrm{c}(\mathrm{c} 1)[\mathrm{nH}] \mathrm{nn} 2$ & -2.71 & -2.16 & 6.08 & 7.53 & 12.88 & 19.00 & 1.06 & 1.05 \\
\hline S267 & {$[\mathrm{nH}] 1 \mathrm{nc} 2 \mathrm{c}(\mathrm{n} 1) \mathrm{cc}[\mathrm{nH}] 2$} & -3.89 & -2.91 & 3.99 & 4.61 & 12.48 & 13.64 & 0.56 & 0.50 \\
\hline S2672 & c1on $2 \mathrm{c}(\mathrm{c} 1) \mathrm{cnn} 2$ & -0.40 & -0.56 & 7.51 & 6.76 & 18.84 & 15.71 & 0.56 & 0.52 \\
\hline S2674 & o1cc2c(c1)nn[nH]2 & -1.54 & -1.31 & 5.79 & 7.25 & 14.68 & 17.02 & 0.60 & 0.53 \\
\hline S2677 & $\operatorname{s} 1 \mathrm{cc} 2 \mathrm{c}(\mathrm{c} 1) \mathrm{nn}[\mathrm{nH}] 2$ & -2.05 & -2.00 & 5.52 & 6.56 & 15.00 & 16.58 & 0.56 & 0.48 \\
\hline S2684 & n1nc2c([nH]1)noc2 & 0.58 & 0.52 & 5.74 & 7.06 & 10.64 & 14.05 & 0.44 & 0.37 \\
\hline S269 & c1cc2c([nH]1)nno2 & -1.22 & -1.31 & 7.74 & 6.55 & 17.10 & 15.60 & 0.47 & 0.46 \\
\hline S2690 & $\mathrm{c} 1[\mathrm{nH}] \mathrm{n} 2 \mathrm{c}(\mathrm{n} 1) \mathrm{cnn} 2$ & -0.39 & -0.33 & 11.64 & 9.40 & 22.94 & 18.74 & 0.47 & 0.47 \\
\hline S2691 & $\mathrm{c} 1 \mathrm{nn} 2 \mathrm{c}([\mathrm{nH}] 1) \mathrm{cnn} 2$ & -0.30 & -0.33 & 13.20 & 11.40 & 26.86 & 23.60 & 0.39 & 0.41 \\
\hline
\end{tabular}




\begin{tabular}{|c|c|c|c|c|c|c|c|c|c|}
\hline S2692 & c1on $2 \mathrm{c}(\mathrm{n} 1) \mathrm{cnn} 2$ & 1.37 & 1.27 & 9.30 & 7.39 & 18.04 & 14.27 & 0.29 & 0.32 \\
\hline S2695 & $\mathrm{c} 1 \mathrm{nn} 2 \mathrm{c}(\mathrm{s} 1) \mathrm{cnn} 2$ & 0.57 & 0.58 & 8.47 & 7.37 & 18.96 & 16.35 & 0.64 & 0.56 \\
\hline S271 & c1cn2c(c1)onn2 & -0.73 & -0.56 & 2.01 & 6.65 & 5.67 & 15.49 & 0.49 & 0.61 \\
\hline S272 & $\mathrm{c} 1 \mathrm{cc} 2 \mathrm{c}([\mathrm{nH}] 1) \operatorname{snn} 2$ & -2.11 & -2.00 & 8.61 & 7.09 & 19.23 & 17.69 & 0.49 & 0.45 \\
\hline S273 & $\mathrm{c} 1 \mathrm{cc} 2 \mathrm{c}([\mathrm{nH}] 1) \mathrm{nns} 2$ & -2.07 & -2.00 & 6.19 & 5.75 & 15.90 & 14.91 & 0.77 & 0.79 \\
\hline S2740 & $\operatorname{c1ccc} 2 \mathrm{c}(\mathrm{c} 1)[\mathrm{nH}] \mathrm{nn} 2$ & -2.37 & -2.53 & 5.70 & 6.77 & 15.86 & 17.59 & 0.38 & 0.39 \\
\hline S2741 & $\operatorname{c} 1 \mathrm{ccc} 2 \mathrm{n}(\mathrm{c} 1) \mathrm{nnc} 2$ & -1.29 & -1.78 & 5.00 & 5.89 & 16.54 & 15.82 & 0.62 & 0.61 \\
\hline S2742 & $\mathrm{c} 1 \mathrm{ncc} 2 \mathrm{c}(\mathrm{c} 1)[\mathrm{nH}] \mathrm{nn} 2$ & -0.38 & -0.70 & 8.63 & 8.58 & 15.92 & 18.29 & 0.41 & 0.45 \\
\hline S2743 & $\mathrm{c} 1 \mathrm{ncc} 2 \mathrm{c}(\mathrm{c} 1) \mathrm{nn}[\mathrm{nH}] 2$ & -0.46 & -0.70 & 8.10 & 7.17 & 15.03 & 15.66 & 0.62 & 0.61 \\
\hline S2744 & $\mathrm{c} 1 \mathrm{cnc} 2 \mathrm{c}(\mathrm{c} 1) \mathrm{nn}[\mathrm{nH}] 2$ & -0.82 & -0.70 & 3.38 & 6.43 & 11.34 & 14.71 & 0.32 & 0.36 \\
\hline S2745 & $\mathrm{c} 1 \mathrm{cnc} 2 \mathrm{c}(\mathrm{c} 1)[\mathrm{nH}] \mathrm{nn} 2$ & -0.56 & -0.70 & 8.56 & 10.31 & 20.06 & 21.91 & 0.78 & 0.77 \\
\hline S2746 & $\mathrm{n} 1 \mathrm{ccn} 2 \mathrm{c}(\mathrm{c} 1) \mathrm{cnn} 2$ & 0.60 & 0.05 & 4.31 & 6.94 & 12.47 & 14.81 & 0.49 & 0.53 \\
\hline S2747 & $\operatorname{clncn} 2 \mathrm{c}(\mathrm{c} 1) \mathrm{cnn} 2$ & 0.60 & 0.05 & 5.78 & 7.41 & 14.11 & 16.01 & 0.81 & 0.78 \\
\hline S275 & $\mathrm{c} 1 \mathrm{cn} 2 \mathrm{c}(\mathrm{c} 1) \operatorname{snn} 2$ & -1.64 & -1.25 & 1.28 & 5.90 & 6.00 & 14.89 & 0.38 & 0.41 \\
\hline S2750 & $\mathrm{c} 1 \mathrm{nnc} 2 \mathrm{c}(\mathrm{c} 1)[\mathrm{nH}] \mathrm{nn} 2$ & 1.52 & 1.13 & 11.98 & 13.15 & 21.91 & 24.86 & 0.31 & 0.34 \\
\hline S2751 & $\mathrm{c} 1 \mathrm{nnc} 2 \mathrm{c}(\mathrm{c} 1) \mathrm{nn}[\mathrm{nH}] 2$ & 1.17 & 1.13 & 5.78 & 7.85 & 11.07 & 15.03 & 0.46 & 0.52 \\
\hline S2754 & $\mathrm{n} 1 \mathrm{ncc} 2 \mathrm{c}(\mathrm{c} 1)[\mathrm{nH}] \mathrm{nn} 2$ & 1.73 & 1.13 & 11.48 & 10.00 & 19.22 & 18.61 & 0.47 & 0.50 \\
\hline S2755 & $\mathrm{n} 1 \mathrm{ncc} 2 \mathrm{n}(\mathrm{c} 1) \mathrm{nnc} 2$ & 2.72 & 1.88 & 7.36 & 9.47 & 11.83 & 17.24 & 0.32 & 0.38 \\
\hline S2756 & $\mathrm{O}=\mathrm{c} 1[\mathrm{nH}] \operatorname{ccc} 2 \mathrm{c} 1 \mathrm{nn}[\mathrm{nH}] 2$ & -0.09 & 0.07 & 8.89 & 12.18 & 22.19 & 26.29 & 0.32 & 0.36 \\
\hline S2757 & $\mathrm{O}=\mathrm{c} 1[\mathrm{nH}] \operatorname{ccc} 2 \mathrm{c} 1[\mathrm{nH}] \mathrm{nn} 2$ & 0.16 & 0.07 & 7.45 & 9.50 & 15.27 & 18.27 & 0.63 & 0.60 \\
\hline S2758 & $\mathrm{O}=\mathrm{c} 1 \mathrm{ccc} 2 \mathrm{c}([\mathrm{nH}] 1)[\mathrm{nH}] \mathrm{nn} 2$ & 1.00 & 0.07 & 11.33 & 11.13 & 18.32 & 20.36 & 0.54 & 0.50 \\
\hline S2759 & $\mathrm{O}=\mathrm{c} 1 \mathrm{ccc} 2 \mathrm{c}([\mathrm{nH}] 1) \mathrm{nn}[\mathrm{nH}] 2$ & 0.89 & 0.07 & 10.61 & 8.34 & 19.71 & 18.36 & 0.65 & 0.65 \\
\hline S276 & $\mathrm{O}=\mathrm{c} 1 \mathrm{c}(=\mathrm{O}) \mathrm{c} 2 \mathrm{c}(\mathrm{c} 1=\mathrm{O})[\mathrm{nH}] \mathrm{cc} 2$ & 2.73 & 3.71 & 12.52 & 12.09 & 21.46 & 24.93 & 0.60 & 0.52 \\
\hline S2760 & $\mathrm{O}=\mathrm{c} 1[\mathrm{nH}] \operatorname{ccn} 2 \mathrm{c} 1 \mathrm{cnn} 2$ & 0.85 & 0.82 & 10.35 & 9.52 & 19.41 & 18.92 & 0.55 & 0.55 \\
\hline S2761 & $\mathrm{O}=\mathrm{c} 1[\mathrm{nH}] \mathrm{ccc} 2 \mathrm{n} 1 \mathrm{nnc} 2$ & 0.66 & 0.82 & 10.80 & 10.19 & 22.79 & 22.21 & 0.38 & 0.35 \\
\hline S2763 & $\mathrm{O}=\mathrm{c} 1 \mathrm{ccn} 2 \mathrm{c}([\mathrm{nH}] 1) \mathrm{cnn} 2$ & 1.94 & 0.82 & 6.93 & 9.32 & 13.85 & 18.11 & 0.35 & 0.39 \\
\hline S2766 & $\mathrm{O}=\mathrm{c} 1 \mathrm{occc} 2 \mathrm{c} 1 \mathrm{nn}[\mathrm{nH}] 2$ & 1.50 & 1.67 & 11.65 & 13.17 & 23.06 & 26.08 & 0.40 & 0.34 \\
\hline S2767 & $\mathrm{O}=\mathrm{c} 1 \mathrm{occc} 2 \mathrm{c} 1[\mathrm{nH}] \mathrm{nn} 2$ & 1.76 & 1.67 & 5.28 & 8.35 & 10.22 & 15.53 & 0.96 & 0.90 \\
\hline S2778 & $\mathrm{O}=\mathrm{c} 1 \mathrm{ccc} 2 \mathrm{c}(\mathrm{s} 1)[\mathrm{nH}] \mathrm{nn} 2$ & 1.19 & 0.98 & 8.49 & 8.20 & 14.03 & 16.12 & 0.41 & 0.44 \\
\hline S278 & $\mathrm{o} 1 \mathrm{nc} 2 \mathrm{c}(\mathrm{n} 1) \mathrm{cc}[\mathrm{nH}] 2$ & -0.99 & -1.31 & 8.53 & 5.93 & 17.86 & 13.43 & 0.54 & 0.48 \\
\hline S2786 & $\mathrm{c} 1 \mathrm{ncc} 2 \mathrm{c}(\mathrm{n} 1) \mathrm{nn}[\mathrm{nH}] 2$ & 1.37 & 1.13 & 11.06 & 10.12 & 19.21 & 18.69 & 0.57 & 0.51 \\
\hline
\end{tabular}




\begin{tabular}{|c|c|c|c|c|c|c|c|c|c|}
\hline S2787 & $\operatorname{c1ncc} 2 \mathrm{c}(\mathrm{n} 1)[\mathrm{nH}] \mathrm{nn} 2$ & 1.18 & 1.13 & 6.38 & 7.65 & 11.29 & 14.11 & 0.61 & 0.56 \\
\hline S279 & $\operatorname{s} 1 \mathrm{nc} 2 \mathrm{c}(\mathrm{n} 1) \mathrm{cc}[\mathrm{nH}] 2$ & -2.20 & -2.00 & 6.30 & 5.03 & 13.72 & 12.42 & 0.55 & 0.51 \\
\hline S2790 & $\mathrm{n} 1 \mathrm{nnn} 2 \mathrm{c}(\mathrm{c} 1)[\mathrm{nH}] \mathrm{nn} 2$ & 3.60 & 2.96 & 14.82 & 13.98 & 22.95 & 23.89 & 0.46 & 0.43 \\
\hline S2791 & $\mathrm{n} 1 \mathrm{nnc} 2 \mathrm{c}(\mathrm{c} 1) \mathrm{nn}[\mathrm{nH}] 2$ & 3.30 & 2.96 & 9.46 & 10.09 & 15.45 & 16.68 & 0.44 & 0.40 \\
\hline S2794 & $\mathrm{O}=\mathrm{c} 1[\mathrm{nH}] \mathrm{ncc} 2 \mathrm{c} 1 \mathrm{nn}[\mathrm{nH}] 2$ & 1.70 & 1.90 & 12.07 & 10.78 & 22.95 & 20.78 & 0.32 & 0.35 \\
\hline S2795 & $\mathrm{O}=\mathrm{c} 1[\mathrm{nH}] \mathrm{ncc} 2 \mathrm{c} 1[\mathrm{nH}] \mathrm{nn} 2$ & 2.04 & 1.90 & 5.86 & 9.52 & 10.04 & 15.39 & 0.41 & 0.47 \\
\hline S2796 & $\mathrm{O}=\mathrm{c} 1[\mathrm{nH}] \mathrm{ncn} 2 \mathrm{c} 1 \mathrm{cnn} 2$ & 2.69 & 2.65 & 7.93 & 9.24 & 13.12 & 15.51 & 0.44 & 0.39 \\
\hline S2797 & $\mathrm{O}=\mathrm{c} 1[\mathrm{nH}] \mathrm{ncc} 2 \mathrm{n} 1 \mathrm{nnc} 2$ & 2.49 & 2.65 & 8.40 & 9.44 & 16.65 & 17.61 & 0.31 & 0.32 \\
\hline S28 & slcnnc1 & -0.45 & -0.66 & 4.08 & 3.98 & 12.71 & 12.73 & 0.72 & 0.64 \\
\hline $\mathbf{S 2 8 0 0}$ & $\mathrm{O}=\mathrm{c} 1 \mathrm{ncc} 2 \mathrm{c}([\mathrm{nH}] 1) \mathrm{nn}[\mathrm{nH}] 2$ & 2.65 & 1.90 & 13.03 & 10.52 & 23.65 & 20.61 & 0.34 & 0.37 \\
\hline S2801 & $\mathrm{O}=\mathrm{c} 1 \mathrm{ncc} 2 \mathrm{c}([\mathrm{nH}] 1)[\mathrm{nH}] \mathrm{nn} 2$ & 2.88 & 1.90 & 14.27 & 14.73 & 23.55 & 25.23 & 0.55 & 0.50 \\
\hline S2802 & $\mathrm{O}=\mathrm{c} 1 \mathrm{ncn} 2 \mathrm{c}([\mathrm{nH}] 1) \mathrm{cnn} 2$ & 3.73 & 2.65 & 9.67 & 12.62 & 15.94 & 22.47 & 0.48 & 0.55 \\
\hline S281 & $\mathrm{O}=\mathrm{c} 1[\mathrm{nH}] \mathrm{n} 2 \mathrm{c}([\mathrm{nH}] 1) \mathrm{ccc} 2$ & -2.44 & -3.22 & 4.62 & 5.82 & 12.94 & 18.21 & 0.51 & 0.48 \\
\hline $\mathbf{S 2 8 3 1}$ & $\mathrm{O}=\mathrm{c} 1 \mathrm{cnc} 2 \mathrm{c}([\mathrm{nH}] 1) \mathrm{nn}[\mathrm{nH}] 2$ & 2.38 & 1.90 & 7.71 & 8.50 & 13.53 & 15.44 & 0.39 & 0.44 \\
\hline $\mathbf{S 2 8 3 3}$ & $\mathrm{O}=\mathrm{c} 1 \mathrm{cnn} 2 \mathrm{c}([\mathrm{nH}] 1) \mathrm{cnn} 2$ & 3.47 & 2.65 & 10.48 & 12.04 & 18.00 & 20.44 & 0.58 & 0.53 \\
\hline S285 & $\mathrm{O}=\mathrm{c} 1[\mathrm{nH}] \mathrm{n} 2 \mathrm{c}(\mathrm{o} 1) \mathrm{ccc} 2$ & -0.94 & -1.62 & 8.09 & 8.31 & 15.98 & 19.11 & 0.57 & 0.52 \\
\hline $\mathbf{S 2 8 6 0}$ & $\mathrm{O}=\mathrm{c} 1 \mathrm{cc}[\mathrm{nH}] \mathrm{c} 2 \mathrm{c} 1[\mathrm{nH}] \mathrm{nn} 2$ & 0.06 & 0.07 & 8.80 & 8.49 & 17.57 & 18.31 & 0.55 & 0.51 \\
\hline S2861 & $\mathrm{O}=\mathrm{c} 1 \mathrm{cc}[\mathrm{nH}] \mathrm{c} 2 \mathrm{c} 1 \mathrm{nn}[\mathrm{nH}] 2$ & -0.10 & 0.07 & 15.73 & 17.10 & 31.69 & 34.07 & 0.76 & 0.75 \\
\hline S2862 & $\mathrm{O}=\mathrm{c} 1 \mathrm{cc}[\mathrm{nH}] \mathrm{c} 2 \mathrm{n} 1 \mathrm{nnc} 2$ & 0.70 & 0.82 & 14.19 & 13.12 & 27.33 & 27.83 & 0.46 & 0.54 \\
\hline S2864 & $\mathrm{O}=\mathrm{c} 1 \mathrm{nc}[\mathrm{nH}] \mathrm{c} 2 \mathrm{c} 1[\mathrm{nH}] \mathrm{nn} 2$ & 1.93 & 1.90 & 10.83 & 10.67 & 20.59 & 20.55 & 0.31 & 0.31 \\
\hline S2865 & $\mathrm{O}=\mathrm{c} 1 \mathrm{nc}[\mathrm{nH}] \mathrm{c} 2 \mathrm{c} 1 \mathrm{nn}[\mathrm{nH}] 2$ & 1.76 & 1.90 & 18.14 & 20.70 & 33.54 & 38.94 & 0.42 & 0.46 \\
\hline S2868 & $\mathrm{c} 1 \mathrm{cnc} 2 \mathrm{c}(\mathrm{n} 1)[\mathrm{nH}] \mathrm{nn} 2$ & 0.98 & 1.13 & 6.28 & 8.95 & 14.74 & 16.79 & 0.36 & 0.41 \\
\hline S2869 & $\mathrm{c} 1 \mathrm{cnc} 2 \mathrm{n}(\mathrm{n} 1) \mathrm{nnc} 2$ & 1.73 & 1.88 & 9.69 & 8.23 & 18.98 & 15.31 & 0.38 & 0.42 \\
\hline S2870 & $\mathrm{c} 1 \mathrm{nnc} 2 \mathrm{c}(\mathrm{n} 1) \mathrm{nn}[\mathrm{nH}] 2$ & 2.98 & 2.96 & 9.19 & 9.78 & 13.51 & 15.81 & 0.47 & 0.48 \\
\hline S2871 & $\mathrm{c} 1 \mathrm{nnc} 2 \mathrm{c}(\mathrm{n} 1)[\mathrm{nH}] \mathrm{nn} 2$ & 3.07 & 2.96 & 10.08 & 11.19 & 15.92 & 18.44 & 0.57 & 0.51 \\
\hline S2874 & $\mathrm{O}=\mathrm{c} 1[\mathrm{nH}] \mathrm{cnc} 2 \mathrm{c} 1[\mathrm{nH}] \mathrm{nn} 2$ & 1.96 & 1.90 & 9.88 & 12.58 & 18.63 & 20.47 & 0.44 & 0.49 \\
\hline S2875 & $\mathrm{O}=\mathrm{c} 1[\mathrm{nH}] \mathrm{cnc} 2 \mathrm{c} 1 \mathrm{nn}[\mathrm{nH}] 2$ & 1.43 & 1.90 & 10.43 & 11.38 & 20.69 & 21.29 & 0.39 & 0.42 \\
\hline S2876 & $\mathrm{O}=\mathrm{c} 1[\mathrm{nH}] \mathrm{cnc} 2 \mathrm{n} 1 \mathrm{nnc} 2$ & 2.20 & 2.65 & 13.22 & 10.86 & 23.05 & 19.44 & 0.43 & 0.41 \\
\hline S2877 & $\mathrm{O}=\mathrm{c} 1[\mathrm{nH}] \mathrm{cnn} 2 \mathrm{c} 1 \mathrm{cnn} 2$ & 2.44 & 2.65 & 13.00 & 11.28 & 21.99 & 19.17 & 0.60 & 0.70 \\
\hline $\mathbf{S 2 8 8 3}$ & $\mathrm{O}=\mathrm{c} 1 \mathrm{ocnc} 2 \mathrm{c} 1 \mathrm{nn}[\mathrm{nH}] 2$ & 2.99 & 3.50 & 9.03 & 11.47 & 17.02 & 19.83 & 0.63 & 0.66 \\
\hline S289 & $\mathrm{O}=\mathrm{c} 1[\mathrm{nH}] \mathrm{n} 2 \mathrm{c}(\mathrm{s} 1) \mathrm{ccc} 2$ & -1.69 & -2.31 & 6.73 & 7.34 & 14.13 & 17.96 & 0.83 & 0.82 \\
\hline
\end{tabular}




\begin{tabular}{|c|c|c|c|c|c|c|c|c|c|}
\hline $\mathbf{S 2 9 0}$ & $\operatorname{c} 1 \operatorname{ccc} 2 \mathrm{c}(\mathrm{c} 1)[\mathrm{nH}] \mathrm{cc} 2$ & -6.17 & -6.19 & 3.51 & 2.46 & 15.45 & 14.22 & 0.58 & 0.52 \\
\hline S291 & $\operatorname{c} 1 \operatorname{ccc} 2 n(c 1) \operatorname{ccc} 2$ & -5.15 & -5.44 & 1.12 & 1.84 & 11.47 & 12.89 & 0.56 & 0.52 \\
\hline $\mathbf{S 2 9 2}$ & c1ncc $2 \mathrm{c}(\mathrm{c} 1)[\mathrm{nH}] \mathrm{cc} 2$ & -4.14 & -4.36 & 6.31 & 4.65 & 17.77 & 15.75 & 0.57 & 0.53 \\
\hline S2925 & $\mathrm{O}=\mathrm{c} 1 \mathrm{coc} 2 \mathrm{c} 1 \mathrm{nn}[\mathrm{nH}] 2$ & 1.28 & 1.67 & 10.62 & 11.44 & 22.57 & 24.36 & 0.37 & 0.35 \\
\hline S2926 & $\mathrm{O}=\mathrm{c} 1 \mathrm{ccoc} 2 \mathrm{n} 1 \mathrm{nnc} 2$ & 2.11 & 2.42 & 10.76 & 9.70 & 20.78 & 20.26 & 0.43 & 0.50 \\
\hline S2929 & $\mathrm{O}=\mathrm{c} 1 \mathrm{ncoc} 2 \mathrm{c} 1 \mathrm{nn}[\mathrm{nH}] 2$ & 3.06 & 3.50 & 13.18 & 14.15 & 24.39 & 27.98 & 0.46 & 0.47 \\
\hline $\mathbf{S 2 9 3}$ & $\mathrm{c} 1 \mathrm{ncc} 2 \mathrm{c}(\mathrm{c} 1) \mathrm{cc}[\mathrm{nH}] 2$ & -4.18 & -4.36 & 5.84 & 3.90 & 16.64 & 14.60 & 0.46 & 0.44 \\
\hline S2935 & $\mathrm{O}=\mathrm{c} 1 \mathrm{ccsc} 2 \mathrm{n} 1 \mathrm{nnc} 2$ & 1.42 & 1.73 & 9.57 & 9.10 & 19.98 & 20.15 & 0.39 & 0.42 \\
\hline S2936 & $\mathrm{O}=\mathrm{c} 1 \mathrm{cn}[\mathrm{nH}] \mathrm{c} 2 \mathrm{c} 1[\mathrm{nH}] \mathrm{nn} 2$ & 1.87 & 1.90 & 5.87 & 8.51 & 11.69 & 15.42 & 0.47 & 0.50 \\
\hline $\mathbf{S 2 9 3 7}$ & $\mathrm{O}=\mathrm{c} 1 \mathrm{cn}[\mathrm{nH}] \mathrm{c} 2 \mathrm{c} 1 \mathrm{nn}[\mathrm{nH}] 2$ & 1.66 & 1.90 & 15.03 & 15.70 & 27.28 & 28.56 & 0.56 & 0.51 \\
\hline S2938 & $\mathrm{O}=\mathrm{c} 1 \mathrm{cn}[\mathrm{nH}] \mathrm{c} 2 \mathrm{n} 1 \mathrm{nnc} 2$ & 2.43 & 2.65 & 12.02 & 12.38 & 21.46 & 23.22 & 0.48 & 0.54 \\
\hline S294 & $\mathrm{c} 1 \mathrm{cnc} 2 \mathrm{c}(\mathrm{c} 1) \mathrm{cc}[\mathrm{nH}] 2$ & -4.69 & -4.36 & 2.07 & 2.88 & 10.73 & 13.01 & 0.51 & 0.44 \\
\hline S2946 & $\mathrm{O}=\mathrm{c} 1 \mathrm{cnsc} 2 \mathrm{n} 1 \mathrm{nnc} 2$ & 3.18 & 3.56 & 9.13 & 9.80 & 16.35 & 17.45 & 0.36 & 0.38 \\
\hline $\mathbf{S 2 9 5}$ & $\operatorname{c} 1 \mathrm{cnc} 2 \mathrm{c}(\mathrm{c} 1)[\mathrm{nH}] \mathrm{cc} 2$ & -4.62 & -4.36 & 5.93 & 4.93 & 17.94 & 16.18 & 0.35 & 0.37 \\
\hline S296 & $\mathrm{n} 1 \mathrm{ccn} 2 \mathrm{c}(\mathrm{c} 1) \operatorname{ccc} 2$ & -3.17 & -3.61 & 3.07 & 3.93 & 10.79 & 14.18 & 0.42 & 0.47 \\
\hline S297 & $\mathrm{c} 1 \mathrm{ncn} 2 \mathrm{c}(\mathrm{c} 1) \operatorname{ccc} 2$ & -3.21 & -3.61 & 1.75 & 3.73 & 8.16 & 13.91 & 0.43 & 0.41 \\
\hline $\mathbf{S 2 9 8}$ & $\mathrm{c} 1 \mathrm{cnn} 2 \mathrm{c}(\mathrm{c} 1) \mathrm{ccc} 2$ & -3.96 & -3.61 & 1.85 & 2.99 & 10.17 & 12.90 & 0.71 & 0.63 \\
\hline $\mathbf{S 2 9 9}$ & $\mathrm{c} 1 \mathrm{ccn} 2 \mathrm{c}(\mathrm{n} 1) \operatorname{ccc} 2$ & -3.66 & -3.61 & 4.37 & 3.73 & 14.03 & 13.91 & 0.69 & 0.61 \\
\hline S3 & $\operatorname{c} 1 \operatorname{ccc} 1$ & -4.49 & -4.32 & -0.85 & 0.71 & 6.99 & 10.30 & 0.70 & 0.65 \\
\hline S300 & $\mathrm{c} 1 \mathrm{nnn} 2 \mathrm{c}(\mathrm{c} 1)[\mathrm{nH}] \mathrm{cc} 2$ & -2.47 & -2.53 & 9.10 & 8.14 & 22.86 & 19.96 & 0.62 & 0.58 \\
\hline S301 & $\mathrm{c} 1 \mathrm{nnn} 2 \mathrm{c}(\mathrm{c} 1) \mathrm{cc}[\mathrm{nH}] 2$ & -2.57 & -2.53 & 5.39 & 5.34 & 17.12 & 15.63 & 0.59 & 0.62 \\
\hline S302 & $\mathrm{n} 1 \mathrm{ccn} 2 \mathrm{c}(\mathrm{n} 1) \operatorname{ccc} 2$ & -1.63 & -1.78 & 6.69 & 6.85 & 16.92 & 17.44 & 0.48 & 0.52 \\
\hline S303 & c1nnn $2 \mathrm{c}(\mathrm{c} 1) \operatorname{ccc} 2$ & -2.05 & -1.78 & 3.54 & 5.90 & 10.91 & 16.17 & 0.50 & 0.52 \\
\hline S304 & $\mathrm{n} 1 \mathrm{ncc} 2 \mathrm{c}(\mathrm{c} 1)[\mathrm{nH}] \mathrm{cc} 2$ & -1.95 & -2.53 & 9.02 & 7.12 & 22.80 & 18.38 & 0.63 & 0.54 \\
\hline S305 & $\mathrm{n} 1 \mathrm{ncc} 2 \mathrm{n}(\mathrm{c} 1) \operatorname{ccc} 2$ & -0.98 & -1.78 & 4.99 & 6.85 & 15.17 & 17.44 & 0.32 & 0.33 \\
\hline S306 & $\mathrm{O}=\mathrm{c} 1[\mathrm{nH}] \operatorname{ccc} 2 \mathrm{c} 1 \mathrm{cc}[\mathrm{nH}] 2$ & -4.03 & -3.59 & 6.32 & 6.27 & 20.67 & 18.96 & 0.45 & 0.47 \\
\hline S3066 & s1cc2c(c1)nno2 & -0.23 & -0.40 & 3.84 & 4.86 & 9.90 & 12.43 & 0.53 & 0.49 \\
\hline S307 & $\mathrm{O}=\mathrm{c} 1[\mathrm{nH}] \mathrm{ccc} 2 \mathrm{c} 1[\mathrm{nH}] \mathrm{cc} 2$ & -3.85 & -3.59 & 2.47 & 4.85 & 12.98 & 15.43 & 0.37 & 0.38 \\
\hline S309 & $\mathrm{O}=\mathrm{c} 1 \mathrm{ccc} 2 \mathrm{c}([\mathrm{nH}] 1) \mathrm{cc}[\mathrm{nH}] 2$ & -2.83 & -3.59 & 7.97 & 5.65 & 22.35 & 18.02 & 0.64 & 0.64 \\
\hline S310 & $\mathrm{O}=\mathrm{c} 1[\mathrm{nH}] \operatorname{ccn} 2 \mathrm{c} 1 \mathrm{ccc} 2$ & -3.16 & -2.84 & 5.56 & 5.13 & 17.35 & 16.50 & 0.36 & 0.38 \\
\hline $\mathbf{S 3 1 0 3}$ & $\operatorname{c1ccc} 2 \mathrm{c}(\mathrm{c} 1) \mathrm{onn} 2$ & -0.58 & -0.93 & 4.00 & 5.18 & 10.79 & 13.47 & 0.36 & 0.34 \\
\hline
\end{tabular}




\begin{tabular}{|c|c|c|c|c|c|c|c|c|c|}
\hline S3104 & c1ncc2c(c1)onn2 & 1.34 & 0.90 & 4.81 & 6.22 & 8.48 & 13.09 & 0.62 & 0.66 \\
\hline S3105 & c1ncc2c(c1)nno2 & 1.33 & 0.90 & 4.15 & 5.81 & 7.68 & 11.88 & 0.38 & 0.36 \\
\hline S3106 & c1cnc2c(c1)nno2 & 1.24 & 0.90 & 6.48 & 6.46 & 11.99 & 12.87 & 0.61 & 0.65 \\
\hline S3107 & c1cnc2c(c1)onn2 & 1.15 & 0.90 & 6.81 & 7.58 & 12.88 & 16.19 & 0.78 & 0.79 \\
\hline S311 & $\mathrm{O}=\mathrm{c} 1[\mathrm{nH}] \operatorname{ccc} 2 \mathrm{n} 1 \mathrm{ccc} 2$ & -3.16 & -2.84 & 5.40 & 4.53 & 14.10 & 15.31 & 0.58 & 0.64 \\
\hline S312 & $\mathrm{O}=\mathrm{c} 1 \mathrm{ccc} 2 \mathrm{n}([\mathrm{nH}] 1) \operatorname{ccc} 2$ & -1.82 & -2.84 & 4.33 & 6.18 & 14.23 & 17.51 & 0.56 & 0.57 \\
\hline S3129 & c1ncc $2 \mathrm{c}(\mathrm{n} 1) \mathrm{nno} 2$ & 3.08 & 2.73 & 7.22 & 7.62 & 9.80 & 13.31 & 0.57 & 0.51 \\
\hline S313 & $\mathrm{O}=\mathrm{c} 1 \mathrm{ccn} 2 \mathrm{c}([\mathrm{nH}] 1) \mathrm{ccc} 2$ & -1.78 & -2.84 & 5.03 & 5.57 & 15.11 & 17.09 & 0.64 & 0.57 \\
\hline S3130 & c1ncc2c(n1)onn2 & 3.16 & 2.73 & 6.93 & 6.91 & 9.14 & 11.19 & 0.48 & 0.43 \\
\hline S316 & $\mathrm{O}=\mathrm{c} 1 \mathrm{occc} 2 \mathrm{c} 1 \mathrm{cc}[\mathrm{nH}] 2$ & -2.36 & -1.99 & 8.89 & 7.82 & 22.36 & 19.55 & 0.70 & 0.66 \\
\hline S317 & $\mathrm{O}=\mathrm{c} 1 \mathrm{occc} 2 \mathrm{c} 1[\mathrm{nH}] \mathrm{cc} 2$ & -2.17 & -1.99 & 4.44 & 5.28 & 14.98 & 14.90 & 0.56 & 0.52 \\
\hline S3176 & c1cnc $2 \mathrm{c}(\mathrm{n} 1) \mathrm{onn} 2$ & 2.94 & 2.73 & 7.66 & 7.84 & 11.28 & 13.34 & 0.42 & 0.45 \\
\hline S328 & $\mathrm{O}=\mathrm{c} 1 \mathrm{ccc} 2 \mathrm{c}(\mathrm{s} 1)[\mathrm{nH}] \mathrm{cc} 2$ & -2.44 & -2.68 & 6.80 & 5.26 & 17.92 & 16.07 & 0.67 & 0.62 \\
\hline S3348 & $\mathrm{O}=\mathrm{c} 1 \mathrm{ccn} 2 \mathrm{n} 1 \mathrm{cccc} 2$ & -1.88 & -2.09 & 7.09 & 4.86 & 21.27 & 16.12 & 0.61 & 0.60 \\
\hline S3359 & $\mathrm{O}=\mathrm{c} 1 \mathrm{ccn} 2 \mathrm{n} 1 \mathrm{cc}[\mathrm{nH}] \mathrm{c} 2=\mathrm{O}$ & 0.13 & 0.51 & 8.08 & 7.04 & 18.66 & 16.52 & 0.59 & 0.52 \\
\hline S336 & $\operatorname{c1ncc} 2 \mathrm{c}(\mathrm{n} 1) \operatorname{cc}[\mathrm{nH}] 2$ & -2.59 & -2.53 & 8.29 & 5.78 & 17.62 & 15.26 & 0.29 & 0.31 \\
\hline S337 & $\operatorname{c} 1 n c c 2 c(n 1)[n H] c c 2$ & -2.62 & -2.53 & 5.01 & 4.48 & 13.27 & 13.24 & 0.57 & 0.51 \\
\hline S3373 & $\mathrm{O}=\mathrm{c} 1 \mathrm{ccn} 2 \mathrm{n} 1 \mathrm{ccnc} 2$ & 0.06 & -0.26 & 8.71 & 6.67 & 19.92 & 16.20 & 0.79 & 0.75 \\
\hline S3374 & $\mathrm{O}=\mathrm{c} 1 \mathrm{ccn} 2 \mathrm{n} 1 \mathrm{cncc} 2$ & 0.09 & -0.26 & 9.04 & 7.40 & 20.39 & 18.26 & 0.51 & 0.51 \\
\hline S338 & $\mathrm{c} 1 \mathrm{ncn} 2 \mathrm{c}(\mathrm{n} 1) \operatorname{ccc} 2$ & -1.67 & -1.78 & 5.05 & 5.03 & 11.68 & 13.63 & 0.41 & 0.37 \\
\hline S3388 & $\mathrm{O}=\mathrm{c} 1 \operatorname{con} 2 \mathrm{n} 1 \cos 2=\mathrm{O}$ & 1.88 & 2.11 & 6.45 & 8.01 & 13.90 & 15.71 & 0.38 & 0.42 \\
\hline S339 & $\mathrm{n} 1 \mathrm{cnn} 2 \mathrm{c}(\mathrm{c} 1) \mathrm{ccc} 2$ & -1.94 & -1.78 & 1.47 & 4.49 & 6.11 & 12.89 & 0.34 & 0.39 \\
\hline S340 & $\operatorname{c} 1 \mathrm{cc} 2 \mathrm{c}([\mathrm{nH}] 1) \mathrm{cnnn} 2$ & -0.32 & -0.70 & 11.74 & 10.02 & 25.28 & 21.29 & 0.40 & 0.46 \\
\hline S341 & $\operatorname{c} 1 \mathrm{cc} 2 \mathrm{c}([\mathrm{nH}] 1) \mathrm{nnnn} 2$ & -0.39 & -0.70 & 8.51 & 7.96 & 20.93 & 18.11 & 0.39 & 0.43 \\
\hline S342 & $\mathrm{n} 1 \mathrm{ncn} 2 \mathrm{c}(\mathrm{n} 1) \operatorname{ccc} 2$ & 0.54 & 0.05 & 8.12 & 9.17 & 17.98 & 19.41 & 0.66 & 0.61 \\
\hline S343 & $\mathrm{n} 1 \mathrm{nnn} 2 \mathrm{c}(\mathrm{c} 1) \mathrm{ccc} 2$ & 0.17 & 0.05 & 4.57 & 8.43 & 13.05 & 18.40 & 0.32 & 0.36 \\
\hline S3432 & $\mathrm{O}=\mathrm{c} 1 \mathrm{ccn} 2 \mathrm{n} 1 \mathrm{cn}[\mathrm{nH}] \mathrm{c} 2=\mathrm{O}$ & 2.13 & 2.34 & 6.89 & 7.78 & 14.04 & 15.07 & 0.47 & 0.43 \\
\hline S344 & $\mathrm{O}=\mathrm{c} 1[\mathrm{nH}] \mathrm{ncc} 2 \mathrm{c} 1 \mathrm{cc}[\mathrm{nH}] 2$ & -2.13 & -1.76 & 9.30 & 5.92 & 21.80 & 15.75 & 0.45 & 0.47 \\
\hline S345 & $\mathrm{O}=\mathrm{c} 1[\mathrm{nH}] \mathrm{ncc} 2 \mathrm{c} 1[\mathrm{nH}] \mathrm{cc} 2$ & -1.88 & -1.76 & 5.03 & 5.25 & 13.97 & 13.37 & 0.33 & 0.34 \\
\hline S3451 & $\mathrm{O}=\mathrm{c} 1 \mathrm{ccn} 2 \mathrm{n} 1 \mathrm{cnsc} 2=\mathrm{O}$ & 2.99 & 3.25 & 8.35 & 9.32 & 13.10 & 15.23 & 0.52 & 0.49 \\
\hline S346 & $\mathrm{O}=\mathrm{c} 1[\mathrm{nH}] \mathrm{ncn} 2 \mathrm{c} 1 \mathrm{ccc} 2$ & -1.19 & -1.01 & 6.06 & 5.23 & 16.05 & 13.93 & 0.42 & 0.52 \\
\hline
\end{tabular}




\begin{tabular}{|c|c|c|c|c|c|c|c|c|c|}
\hline S347 & $\mathrm{O}=\mathrm{c} 1[\mathrm{nH}] \mathrm{ncc} 2 \mathrm{n} 1 \mathrm{ccc} 2$ & -1.23 & -1.01 & 2.56 & 4.83 & 9.56 & 13.01 & 0.59 & 0.56 \\
\hline S350 & $\mathrm{O}=\mathrm{c} 1 \mathrm{ncc} 2 \mathrm{c}([\mathrm{nH}] 1) \mathrm{cc}[\mathrm{nH}] 2$ & -0.93 & -1.76 & 10.37 & 8.88 & 26.68 & 22.57 & 0.69 & 0.61 \\
\hline S352 & $\mathrm{O}=\mathrm{c} 1 \mathrm{ncn} 2 \mathrm{c}([\mathrm{nH}] 1) \mathrm{ccc} 2$ & 0.09 & -1.01 & 5.95 & 9.24 & 17.59 & 22.28 & 0.63 & 0.63 \\
\hline S353 & $\mathrm{O}=\mathrm{c} 1 \mathrm{ncc} 2 \mathrm{n}([\mathrm{nH}] 1) \operatorname{ccc} 2$ & 0.07 & -1.01 & 7.34 & 10.06 & 19.58 & 22.96 & 0.71 & 0.65 \\
\hline S3534 & s1cc2c(c1)nns2 & -1.05 & -1.09 & 4.06 & 4.16 & 11.36 & 11.91 & 0.51 & 0.57 \\
\hline S3567 & $\operatorname{c} 1 \operatorname{ccc} 2 \mathrm{c}(\mathrm{c} 1) \operatorname{snn} 2$ & -1.33 & -1.62 & 3.77 & 4.47 & 11.70 & 12.94 & 0.69 & 0.64 \\
\hline S3568 & $\mathrm{c} 1 \mathrm{ncc} 2 \mathrm{c}(\mathrm{c} 1) \operatorname{snn} 2$ & 0.63 & 0.21 & 5.66 & 5.56 & 10.64 & 12.70 & 0.42 & 0.43 \\
\hline S3569 & $\mathrm{c} 1 \mathrm{ncc} 2 \mathrm{c}(\mathrm{c} 1) \mathrm{nns} 2$ & 0.57 & 0.21 & 3.92 & 5.09 & 8.52 & 11.31 & 0.40 & 0.47 \\
\hline S3571 & $\mathrm{c} 1 \mathrm{cnc} 2 \mathrm{c}(\mathrm{c} 1) \operatorname{snn} 2$ & 0.34 & 0.21 & 6.95 & 6.94 & 15.61 & 15.86 & 0.58 & 0.58 \\
\hline S3575 & $\mathrm{O}=\mathrm{c} 1[\mathrm{nH}] \mathrm{ccc} 2 \mathrm{c} 1 \mathrm{nns} 2$ & 0.81 & 0.98 & 10.06 & 9.51 & 21.14 & 20.43 & 0.62 & 0.68 \\
\hline S3576 & $\mathrm{O}=\mathrm{c} 1[\mathrm{nH}] \mathrm{ccc} 2 \mathrm{c} 1 \mathrm{snn} 2$ & 0.94 & 0.98 & 9.00 & 8.62 & 15.09 & 16.19 & 0.53 & 0.45 \\
\hline S3578 & $\mathrm{O}=\mathrm{c} 1 \mathrm{ccc} 2 \mathrm{c}([\mathrm{nH}] 1) \mathrm{nns} 2$ & 1.95 & 0.98 & 7.80 & 6.45 & 15.13 & 13.50 & 0.91 & 0.91 \\
\hline S3593 & $\mathrm{c} 1 \mathrm{ncc} 2 \mathrm{c}(\mathrm{n} 1) \mathrm{nn} \mathrm{s} 2$ & 2.26 & 2.04 & 7.63 & 6.97 & 12.96 & 12.93 & 0.39 & 0.43 \\
\hline S3597 & $\mathrm{O}=\mathrm{c} 1[\mathrm{nH}] \mathrm{ncc} 2 \mathrm{c} 1 \mathrm{nns} 2$ & 2.64 & 2.81 & 8.95 & 8.34 & 17.68 & 15.21 & 0.51 & 0.49 \\
\hline S3598 & $\mathrm{O}=\mathrm{c} 1[\mathrm{nH}] \mathrm{ncc} 2 \mathrm{c} 1 \mathrm{snn} 2$ & 2.82 & 2.81 & 6.57 & 7.92 & 10.81 & 12.36 & 0.31 & 0.36 \\
\hline S3622 & $\mathrm{O}=\mathrm{c} 1 \mathrm{cnc} 2 \mathrm{c}([\mathrm{nH}] 1) \mathrm{nnns} 2$ & 3.41 & 2.81 & 7.84 & 8.12 & 12.11 & 12.57 & 0.46 & 0.49 \\
\hline S3643 & $\mathrm{O}=\mathrm{c} 1[\mathrm{nH}] \mathrm{cnc} 2 \mathrm{c} 1 \mathrm{snn} 2$ & 2.61 & 2.81 & 11.35 & 10.63 & 17.79 & 16.99 & 0.52 & 0.43 \\
\hline S3644 & $\mathrm{O}=\mathrm{c} 1[\mathrm{nH}] \mathrm{cnc} 2 \mathrm{c} 1 \mathrm{nns} 2$ & 2.29 & 2.81 & 11.89 & 10.23 & 20.00 & 17.42 & 0.49 & 0.42 \\
\hline S382 & $\mathrm{O}=\mathrm{c} 1 \mathrm{cnc} 2 \mathrm{n}([\mathrm{nH}] 1) \mathrm{ccc} 2$ & -0.41 & -1.01 & 7.06 & 8.57 & 14.06 & 18.48 & 0.54 & 0.49 \\
\hline S383 & $\mathrm{O}=\mathrm{c} 1 \mathrm{cnn} 2 \mathrm{c}([\mathrm{nH}] 1) \mathrm{ccc} 2$ & -0.61 & -1.01 & 4.20 & 7.21 & 10.96 & 17.06 & 0.62 & 0.67 \\
\hline S386 & $\mathrm{O}=\mathrm{c} 1 \mathrm{nnc} 2 \mathrm{n}([\mathrm{nH}] 1) \mathrm{ccc} 2$ & 1.48 & 0.82 & 10.58 & 13.47 & 20.90 & 26.18 & 0.33 & 0.37 \\
\hline S390 & $\mathrm{O}=\mathrm{c} 1[\mathrm{nH}] \mathrm{c}(=\mathrm{O}) \mathrm{c} 2 \mathrm{n}([\mathrm{nH}] 1) \mathrm{ccc} 2$ & 0.35 & -0.24 & 8.39 & 8.02 & 16.04 & 17.77 & 0.84 & 0.87 \\
\hline S391 & $\mathrm{O}=\mathrm{c} 1[\mathrm{nH}] \mathrm{c}(=\mathrm{O}) \mathrm{n} 2 \mathrm{c}([\mathrm{nH}] 1) \mathrm{ccc} 2$ & 0.36 & -0.24 & 5.52 & 6.81 & 10.65 & 16.16 & 0.37 & 0.35 \\
\hline S393 & $\mathrm{O}=\mathrm{c} 1[\mathrm{nH}] \mathrm{n} 2 \operatorname{cccc} 2[\mathrm{nH}] \mathrm{c} 1=\mathrm{O}$ & 0.64 & -0.24 & 7.17 & 11.31 & 17.39 & 27.59 & 0.56 & 0.55 \\
\hline S396 & $\mathrm{O}=\mathrm{c} 1 \mathrm{oc}(=\mathrm{O}) \mathrm{c} 2 \mathrm{n}([\mathrm{nH}] 1) \mathrm{ccc} 2$ & 1.85 & 1.36 & 10.85 & 12.12 & 19.08 & 22.13 & 0.45 & 0.42 \\
\hline S4 & $\operatorname{clccn}[\mathrm{nH}] 1$ & -3.96 & -3.40 & 3.23 & 3.37 & 12.23 & 13.78 & 0.39 & 0.48 \\
\hline $\mathrm{S} 400$ & $\mathrm{O}=\mathrm{c} 1[\mathrm{nH}] \mathrm{n} 2 \operatorname{ccc} 2 \mathrm{oc} 1=\mathrm{O}$ & 1.72 & 1.36 & 9.74 & 13.17 & 17.90 & 27.63 & 0.44 & 0.49 \\
\hline S404 & $\mathrm{O}=\mathrm{c} 1 \mathrm{sc}(=\mathrm{O}) \mathrm{c} 2 \mathrm{n}([\mathrm{nH}] 1) \mathrm{ccc} 2$ & 0.92 & 0.67 & 9.76 & 11.04 & 16.73 & 20.56 & 0.49 & 0.52 \\
\hline S408 & $\mathrm{O}=\mathrm{c} 1[\mathrm{nH}] \mathrm{n} 2 \operatorname{ccc} 2 \mathrm{sc} 1=\mathrm{O}$ & 0.96 & 0.67 & 8.27 & 12.23 & 16.57 & 26.60 & 0.64 & 0.54 \\
\hline S410 & $\mathrm{O}=\mathrm{c} 1 \mathrm{cc}[\mathrm{nH}] \mathrm{c} 2 \mathrm{c} 1[\mathrm{nH}] \mathrm{cc} 2$ & -3.84 & -3.59 & 6.90 & 6.22 & 21.60 & 19.14 & 0.58 & 0.52 \\
\hline $\mathbf{S 4 1 0 0}$ & $\mathrm{O}=\mathrm{c} 1 \mathrm{c}(=\mathrm{O}) \mathrm{c} 2 \mathrm{c}(\mathrm{c} 1=\mathrm{O}) \mathrm{ccc} 2$ & 3.14 & 4.09 & 8.40 & 10.14 & 15.75 & 21.50 & 0.52 & 0.58 \\
\hline
\end{tabular}




\begin{tabular}{|c|c|c|c|c|c|c|c|c|c|}
\hline $\mathbf{S 4 1 0 2}$ & $\mathrm{O}=\mathrm{c} 1 \mathrm{c}(=\mathrm{O}) \mathrm{c}(=\mathrm{O}) \mathrm{c} 2 \mathrm{c} 1 \mathrm{nccc} 2$ & 4.50 & 5.92 & 10.70 & 12.11 & 17.14 & 23.13 & 0.47 & 0.49 \\
\hline S411 & $\mathrm{O}=\mathrm{c} 1 \mathrm{cc}[\mathrm{nH}] \mathrm{c} 2 \mathrm{c} 1 \mathrm{cc}[\mathrm{nH}] 2$ & -3.98 & -3.59 & 11.35 & 10.76 & 29.13 & 26.08 & 0.43 & 0.55 \\
\hline S412 & $\mathrm{O}=\mathrm{c} 1 \mathrm{cc}[\mathrm{nH}] \mathrm{c} 2 \mathrm{n} 1 \mathrm{ccc} 2$ & -3.12 & -2.84 & 8.94 & 7.04 & 20.82 & 20.27 & 0.51 & 0.49 \\
\hline S413 & $\mathrm{O}=\mathrm{c} 1 \mathrm{cc}[\mathrm{nH}] \mathrm{n} 2 \mathrm{c} 1 \mathrm{ccc} 2$ & -3.09 & -2.84 & 10.95 & 8.69 & 25.64 & 22.47 & 0.57 & 0.52 \\
\hline S4138 & $\mathrm{O}=\mathrm{c} 1 \mathrm{c}(=\mathrm{O}) \mathrm{c} 2 \mathrm{c}(\mathrm{c} 1=\mathrm{O}) \mathrm{nccn} 2$ & 5.83 & 7.75 & 12.65 & 13.06 & 17.56 & 22.52 & 0.49 & 0.46 \\
\hline S4139 & $\mathrm{O}=\mathrm{c} 1 \mathrm{c}(=\mathrm{O}) \mathrm{c} 2 \mathrm{c}(\mathrm{c} 1=\mathrm{O}) \mathrm{nncn} 2$ & 7.96 & 9.58 & 17.23 & 14.14 & 19.18 & 21.38 & 0.60 & 0.61 \\
\hline S414 & $\mathrm{O}=\mathrm{c} 1 \mathrm{nc}[\mathrm{nH}] \mathrm{c} 2 \mathrm{c} 1[\mathrm{nH}] \mathrm{cc} 2$ & -1.93 & -1.76 & 8.89 & 9.44 & 24.67 & 23.68 & 0.28 & 0.30 \\
\hline S415 & $\mathrm{O}=\mathrm{c} 1 \mathrm{nc}[\mathrm{nH}] \mathrm{c} 2 \mathrm{c} 1 \mathrm{cc}[\mathrm{nH}] 2$ & -2.10 & -1.76 & 13.55 & 14.74 & 31.75 & 31.79 & 0.56 & 0.49 \\
\hline S416 & $\mathrm{O}=\mathrm{c} 1 \mathrm{nc}[\mathrm{nH}] \mathrm{c} 2 \mathrm{n} 1 \mathrm{ccc} 2$ & -1.28 & -1.01 & 11.20 & 10.72 & 23.79 & 25.46 & 0.49 & 0.42 \\
\hline S417 & $\mathrm{O}=\mathrm{c} 1 \mathrm{nc}[\mathrm{nH}] \mathrm{n} 2 \mathrm{c} 1 \mathrm{ccc} 2$ & -1.20 & -1.01 & 13.14 & 12.57 & 28.15 & 27.92 & 0.49 & 0.56 \\
\hline S418 & $\mathrm{c} 1 \mathrm{cnc} 2 \mathrm{c}(\mathrm{n} 1)[\mathrm{nH}] \mathrm{cc} 2$ & -3.11 & -2.53 & 4.73 & 4.32 & 12.86 & 12.72 & 0.35 & 0.40 \\
\hline S419 & $\mathrm{c} 1 \mathrm{cnc} 2 \mathrm{n}(\mathrm{n} 1) \operatorname{ccc} 2$ & -2.49 & -1.78 & 3.07 & 3.86 & 9.52 & 11.67 & 0.73 & 0.65 \\
\hline $\mathbf{S 4 2 0}$ & $\mathrm{c} 1 \mathrm{nnc} 2 \mathrm{c}(\mathrm{n} 1) \mathrm{cc}[\mathrm{nH}] 2$ & -0.92 & -0.70 & 7.88 & 6.20 & 16.82 & 14.05 & 0.53 & 0.49 \\
\hline S421 & c1nnc2c(n1)[nH]cc2 & -0.88 & -0.70 & 8.39 & 6.94 & 18.82 & 15.20 & 0.61 & 0.67 \\
\hline $\mathbf{S 4 2 3}$ & $\mathrm{n} 1 \mathrm{cnn} 2 \mathrm{c}(\mathrm{n} 1) \operatorname{ccc} 2$ & -0.36 & 0.05 & 3.46 & 6.38 & 10.25 & 13.91 & 0.62 & 0.55 \\
\hline S424 & $\mathrm{O}=\mathrm{c} 1[\mathrm{nH}] \mathrm{cnc} 2 \mathrm{c} 1[\mathrm{nH}] \mathrm{cc} 2$ & -2.34 & -1.76 & 4.04 & 6.86 & 11.62 & 15.26 & 0.75 & 0.72 \\
\hline $\mathbf{S 4 2 5}$ & $\mathrm{O}=\mathrm{c} 1[\mathrm{nH}] \mathrm{cnc} 2 \mathrm{c} 1 \mathrm{cc}[\mathrm{nH}] 2$ & -2.60 & -1.76 & 4.79 & 6.22 & 16.00 & 15.62 & 0.42 & 0.45 \\
\hline S426 & $\mathrm{O}=\mathrm{c} 1[\mathrm{nH}] \mathrm{cnc} 2 \mathrm{n} 1 \mathrm{ccc} 2$ & -1.66 & -1.01 & 7.53 & 5.96 & 13.90 & 14.20 & 0.71 & 0.65 \\
\hline $\mathbf{S 4 2 7}$ & $\mathrm{O}=\mathrm{c} 1[\mathrm{nH}] \mathrm{cnn} 2 \mathrm{c} 1 \mathrm{ccc} 2$ & -1.90 & -1.01 & 7.27 & 5.81 & 15.88 & 14.39 & 0.58 & 0.65 \\
\hline $\mathbf{S 4 2 9 0}$ & $\mathrm{O}=\mathrm{c} 1[\mathrm{nH}] \mathrm{cc} 2 \mathrm{n} 1 \mathrm{cc}[\mathrm{nH}] 2$ & -3.62 & -3.22 & 6.93 & 6.89 & 21.34 & 20.54 & 0.28 & 0.33 \\
\hline $\mathbf{S 4 2 9 7}$ & $\mathrm{O}=\mathrm{c} 1[\mathrm{nH}] \mathrm{c} 2 \mathrm{c}([\mathrm{nH}] 1) \mathrm{csc} 2$ & -2.09 & -3.06 & 2.87 & 4.32 & 11.73 & 14.85 & 0.57 & 0.48 \\
\hline $\mathbf{S 4 3 0 0}$ & $\mathrm{O}=\mathrm{c} 1[\mathrm{nH}] \mathrm{c} 2 \mathrm{c}([\mathrm{nH}] 1) \mathrm{c}[\mathrm{nH}] \mathrm{n} 2$ & -2.07 & -2.14 & 5.56 & 6.91 & 15.86 & 18.49 & 0.81 & 0.86 \\
\hline S432 & $\mathrm{O}=\mathrm{c} 1 \mathrm{locnc} 2 \mathrm{c} 1[\mathrm{nH}] \mathrm{cc} 2$ & -0.66 & -0.16 & 5.88 & 6.39 & 13.70 & 13.49 & 0.59 & 0.51 \\
\hline S433 & $\mathrm{O}=\mathrm{c} 1 \mathrm{ocnc} 2 \mathrm{c} 1 \mathrm{cc}[\mathrm{nH}] 2$ & -0.93 & -0.16 & 7.10 & 6.89 & 17.50 & 14.96 & 0.67 & 0.59 \\
\hline S4331 & $\mathrm{O}=\mathrm{c} 1[\mathrm{nH}] \mathrm{c} 2 \mathrm{c}([\mathrm{nH}] 1) \mathrm{cccc} 2$ & -2.30 & -3.59 & 2.43 & 4.40 & 12.29 & 15.84 & 0.55 & 0.49 \\
\hline S4332 & $\mathrm{O}=\mathrm{c} 1[\mathrm{nH}] \operatorname{cc} 2 \mathrm{n} 1 \mathrm{cccc} 2$ & -2.86 & -2.84 & 3.21 & 4.99 & 14.38 & 16.39 & 0.42 & 0.35 \\
\hline S4333 & $\mathrm{O}=\mathrm{c} 1[\mathrm{nH}] \mathrm{c} 2 \mathrm{c}([\mathrm{nH}] 1) \mathrm{ccnc} 2$ & -0.34 & -1.76 & 5.52 & 5.82 & 12.66 & 15.45 & 0.37 & 0.41 \\
\hline S4334 & $\mathrm{O}=\mathrm{c} 1[\mathrm{nH}] \mathrm{c} 2 \mathrm{c}([\mathrm{nH}] 1) \mathrm{cccn} 2$ & -0.85 & -1.76 & 5.46 & 5.66 & 13.97 & 15.94 & 0.45 & 0.52 \\
\hline S4336 & $\mathrm{O}=\mathrm{c} 1[\mathrm{nH}] \mathrm{cc} 2 \mathrm{n} 1 \mathrm{cncc} 2$ & -0.87 & -1.01 & 5.79 & 7.65 & 14.45 & 18.82 & 0.33 & 0.36 \\
\hline S4344 & $\mathrm{O}=\mathrm{c} 1[\mathrm{nH}] \mathrm{c} 2 \mathrm{c}([\mathrm{nH}] 1) \mathrm{cc}[\mathrm{nH}] \mathrm{c} 2=\mathrm{O}$ & -0.11 & -0.99 & 8.00 & 7.74 & 17.84 & 19.49 & 0.36 & 0.42 \\
\hline S4345 & $\mathrm{O}=\mathrm{c} 1 \mathrm{ccc} 2 \mathrm{c}([\mathrm{nH}] 1)[\mathrm{nH}] \mathrm{c}(=\mathrm{O})[\mathrm{nH}] 2$ & 1.13 & -0.99 & 8.79 & 8.10 & 18.38 & 18.41 & 0.31 & 0.33 \\
\hline
\end{tabular}




\begin{tabular}{|c|c|c|c|c|c|c|c|c|c|}
\hline S4346 & $\mathrm{O}=\mathrm{c} 1[\mathrm{nH}] \mathrm{cc} 2 \mathrm{n} 1 \mathrm{cc}[\mathrm{nH}] \mathrm{c} 2=\mathrm{O}$ & -0.74 & -0.24 & 5.34 & 7.31 & 13.72 & 17.24 & 0.48 & 0.40 \\
\hline S4353 & $\mathrm{O}=\mathrm{c} 1[\mathrm{nH}] \operatorname{cc} 2 \mathrm{n} 1 \mathrm{c} \operatorname{coc} 2=\mathrm{O}$ & 0.98 & 1.36 & 7.72 & 8.78 & 14.58 & 16.97 & 0.39 & 0.43 \\
\hline S4365 & $\mathrm{O}=\mathrm{c} 1[\mathrm{nH}] \mathrm{c} 2 \mathrm{c}([\mathrm{nH}] 1) \mathrm{cncn} 2$ & 1.14 & 0.07 & 7.14 & 6.50 & 12.83 & 14.25 & 0.58 & 0.68 \\
\hline S4371 & $\mathrm{O}=\mathrm{c} 1[\mathrm{nH}] \mathrm{c} 2 \mathrm{c}([\mathrm{nH}] 1) \mathrm{cn}[\mathrm{nH}] \mathrm{c} 2=\mathrm{O}$ & 1.80 & 0.84 & 8.78 & 7.37 & 15.28 & 15.51 & 0.34 & 0.35 \\
\hline S4378 & $\mathrm{O}=\mathrm{c} 1[\mathrm{nH}] \mathrm{cc} 2 \mathrm{c}(\mathrm{n} 1)[\mathrm{nH}] \mathrm{c}(=\mathrm{O})[\mathrm{nH}] 2$ & 2.02 & 0.84 & 11.81 & 10.96 & 23.84 & 23.07 & 0.35 & 0.36 \\
\hline S4395 & $\mathrm{O}=\mathrm{c} 1 \mathrm{cnc} 2 \mathrm{c}([\mathrm{nH}] 1)[\mathrm{nH}] \mathrm{c}(=\mathrm{O})[\mathrm{nH}] 2$ & 2.52 & 0.84 & 11.69 & 9.86 & 18.73 & 18.47 & 0.40 & 0.38 \\
\hline S441 & $\mathrm{O}=\mathrm{c} 1 \mathrm{scnc} 2 \mathrm{c} 1 \mathrm{cc}[\mathrm{nH}] 2$ & -1.78 & -0.85 & 6.48 & 6.03 & 16.31 & 14.01 & 0.53 & 0.56 \\
\hline S4418 & $\mathrm{O}=\mathrm{c} 1[\mathrm{nH}] \mathrm{c} 2 \mathrm{c}([\mathrm{nH}] 1)[\mathrm{nH}] \mathrm{ccc} 2=\mathrm{O}$ & -0.06 & -0.99 & 14.07 & 10.43 & 26.23 & 24.06 & 0.48 & 0.43 \\
\hline S4421 & $\mathrm{O}=\mathrm{c} 1[\mathrm{nH}] \mathrm{c} 2 \mathrm{c}([\mathrm{nH}] 1)[\mathrm{nH}] \mathrm{cnc} 2=\mathrm{O}$ & 1.79 & 0.84 & 16.30 & 13.64 & 29.20 & 27.84 & 0.58 & 0.53 \\
\hline S4424 & $\mathrm{O}=\mathrm{c} 1[\mathrm{nH}] \mathrm{c} 2 \mathrm{c}([\mathrm{nH}] 1) \mathrm{nccn} 2$ & 0.65 & 0.07 & 3.52 & 5.90 & 10.01 & 13.79 & 0.66 & 0.60 \\
\hline S4426 & $\mathrm{O}=\mathrm{c} 1[\mathrm{nH}] \mathrm{c} 2 \mathrm{c}([\mathrm{nH}] 1) \mathrm{ncnn} 2$ & 2.84 & 1.90 & 8.09 & 7.76 & 13.77 & 14.35 & 0.29 & 0.31 \\
\hline S4429 & $\mathrm{O}=\mathrm{c} 1[\mathrm{nH}] \mathrm{c} 2 \mathrm{c}([\mathrm{nH}] 1) \mathrm{nc}[\mathrm{nH}] \mathrm{c} 2=\mathrm{O}$ & 1.30 & 0.84 & 9.63 & 8.54 & 18.47 & 17.46 & 0.66 & 0.59 \\
\hline S444 & $\mathrm{n} 1 \mathrm{nnc} 2 \mathrm{c}(\mathrm{n} 1)[\mathrm{nH}] \mathrm{cc} 2$ & 1.41 & 1.13 & 11.60 & 9.84 & 22.70 & 18.78 & 0.63 & 0.55 \\
\hline S445 & $\mathrm{n} 1 \mathrm{nnc} 2 \mathrm{n}(\mathrm{n} 1) \mathrm{ccc} 2$ & 1.74 & 1.88 & 6.34 & 9.73 & 13.53 & 18.12 & 0.38 & 0.43 \\
\hline S4473 & $\mathrm{O}=\mathrm{c} 1[\mathrm{nH}] \mathrm{c} 2 \mathrm{c}([\mathrm{nH}] 1)[\mathrm{nH}] \mathrm{ncc} 2=\mathrm{O}$ & 1.78 & 0.84 & 13.40 & 10.06 & 22.87 & 20.08 & 0.69 & 0.75 \\
\hline S452 & $\mathrm{O}=\mathrm{c} 1[\mathrm{nH}] \mathrm{nnc} 2 \mathrm{c} 1 \mathrm{cc}[\mathrm{nH}] 2$ & -0.70 & 0.07 & 7.58 & 6.89 & 16.64 & 14.66 & 0.79 & 0.91 \\
\hline S453 & $\mathrm{O}=\mathrm{c} 1[\mathrm{nH}] \mathrm{nnc} 2 \mathrm{c} 1[\mathrm{nH}] \mathrm{cc} 2$ & -0.39 & 0.07 & 6.74 & 8.28 & 13.40 & 15.45 & 0.42 & 0.36 \\
\hline S4536 & $\mathrm{O}=\mathrm{c} 1[\mathrm{nH}] \mathrm{c} 2 \mathrm{c}(\mathrm{o} 1) \csc 2$ & -0.64 & -1.46 & 6.10 & 5.99 & 14.73 & 15.11 & 0.49 & 0.46 \\
\hline S455 & $\mathrm{O}=\mathrm{c} 1[\mathrm{nH}] \mathrm{nnc} 2 \mathrm{n} 1 \mathrm{ccc} 2$ & 0.23 & 0.82 & 3.56 & 7.28 & 7.73 & 14.15 & 0.44 & 0.39 \\
\hline S4575 & $\mathrm{O}=\mathrm{c} 1 \mathrm{occ} 2 \mathrm{n} 1 \mathrm{ccc} 2$ & -0.77 & -1.24 & 4.43 & 5.75 & 14.85 & 15.58 & 0.64 & 0.60 \\
\hline S4579 & $\mathrm{O}=\mathrm{c} 1 \mathrm{oc} 2 \mathrm{c}([\mathrm{nH}] 1) \mathrm{nccc} 2$ & 0.52 & -0.16 & 4.05 & 6.29 & 12.05 & 14.62 & 0.37 & 0.42 \\
\hline S4581 & $\mathrm{O}=\mathrm{c} 1 \mathrm{occ} 2 \mathrm{n} 1 \mathrm{cncc} 2$ & 1.17 & 0.59 & 5.95 & 7.60 & 13.69 & 16.88 & 0.47 & 0.46 \\
\hline $\mathbf{S 4 6 2 0}$ & $\mathrm{O}=\mathrm{c} 1 \mathrm{oc} 2 \mathrm{c}([\mathrm{nH}] 1) \mathrm{ncnc} 2$ & 2.53 & 1.67 & 7.03 & 7.37 & 12.25 & 13.27 & 0.59 & 0.58 \\
\hline S472 & $\mathrm{O}=\mathrm{c} 1 \mathrm{ccoc} 2 \mathrm{c} 1[\mathrm{nH}] \mathrm{cc} 2$ & -2.41 & -1.99 & 3.85 & 4.80 & 14.44 & 14.08 & 0.61 & 0.55 \\
\hline S473 & $\mathrm{O}=\mathrm{c} 1 \mathrm{cooc} 2 \mathrm{c} 1 \mathrm{cc}[\mathrm{nH}] 2$ & -2.65 & -1.99 & 7.85 & 6.25 & 21.53 & 17.97 & 0.45 & 0.36 \\
\hline S474 & $\mathrm{O}=\mathrm{c} 1 \mathrm{ccoc} 2 \mathrm{n} 1 \mathrm{ccc} 2$ & -1.72 & -1.24 & 5.45 & 4.76 & 13.52 & 14.30 & 0.27 & 0.29 \\
\hline S476 & $\mathrm{O}=\mathrm{c} 1 \mathrm{n} \operatorname{coc} 2 \mathrm{c} 1[\mathrm{nH}] \mathrm{cc} 2$ & -0.57 & -0.16 & 6.04 & 7.13 & 17.81 & 17.38 & 0.38 & 0.44 \\
\hline S477 & $\mathrm{O}=\mathrm{c} 1 \mathrm{n} \operatorname{coc} 2 \mathrm{c} 1 \mathrm{cc}[\mathrm{nH}] 2$ & -0.83 & -0.16 & 10.12 & 9.33 & 24.13 & 22.43 & 0.41 & 0.46 \\
\hline S48 & $\operatorname{clcccc} 1$ & -4.74 & -4.85 & -1.41 & 0.73 & 7.36 & 11.22 & 0.37 & 0.40 \\
\hline $\mathbf{S 4 8 0}$ & $\mathrm{O}=\mathrm{c} 1 \mathrm{ccsc} 2 \mathrm{c} 1 \mathrm{cc}[\mathrm{nH}] 2$ & -3.18 & -2.68 & 7.61 & 5.73 & 20.98 & 17.93 & 0.55 & 0.47 \\
\hline S483 & $\mathrm{O}=\mathrm{c} 1 \csc 2 \mathrm{n} 1 \mathrm{ccc} 2$ & -2.26 & -1.93 & 4.57 & 4.09 & 12.72 & 14.00 & 0.50 & 0.46 \\
\hline
\end{tabular}




\begin{tabular}{|c|c|c|c|c|c|c|c|c|c|}
\hline S484 & $\mathrm{O}=\mathrm{c} 1 \mathrm{cn}[\mathrm{nH}] \mathrm{c} 2 \mathrm{c} 1[\mathrm{nH}] \mathrm{cc} 2$ & -1.97 & -1.76 & 5.38 & 6.61 & 17.23 & 17.08 & 0.42 & 0.36 \\
\hline S4845 & $\mathrm{O}=\mathrm{c} 1 \mathrm{scc} 2 \mathrm{n} 1 \mathrm{ccs} 2$ & -1.32 & -1.40 & 4.57 & 4.72 & 13.41 & 13.62 & 0.52 & 0.47 \\
\hline S485 & $\mathrm{O}=\mathrm{c} 1 \mathrm{cn}[\mathrm{nH}] \mathrm{c} 2 \mathrm{c} 1 \mathrm{cc}[\mathrm{nH}] 2$ & -2.14 & -1.76 & 11.87 & 10.41 & 26.38 & 22.87 & 0.46 & 0.52 \\
\hline S486 & $\mathrm{O}=\mathrm{c} 1 \mathrm{cn}[\mathrm{nH}] \mathrm{c} 2 \mathrm{n} 1 \mathrm{ccc} 2$ & -1.31 & -1.01 & 6.69 & 7.34 & 15.24 & 17.97 & 0.67 & 0.60 \\
\hline S4884 & $\mathrm{O}=\mathrm{c} 1 \mathrm{sc} 2 \mathrm{c}([\mathrm{nH}] 1) \mathrm{cncc} 2$ & 0.35 & -0.85 & 6.17 & 5.95 & 12.61 & 13.72 & 0.66 & 0.60 \\
\hline S4885 & $\mathrm{O}=\mathrm{c} 1 \mathrm{sc} 2 \mathrm{c}([\mathrm{nH}] 1) \mathrm{ccnc} 2$ & 0.36 & -0.85 & 7.59 & 6.89 & 13.92 & 14.96 & 0.60 & 0.60 \\
\hline S4887 & $\mathrm{O}=\mathrm{c} 1 \mathrm{sc} 2 \mathrm{c}([\mathrm{nH}] 1) \mathrm{nccc} 2$ & -0.29 & -0.85 & 4.35 & 5.44 & 12.33 & 13.75 & 0.76 & 0.67 \\
\hline S49 & clccenc1 & -2.78 & -3.02 & 2.18 & 2.35 & 10.04 & 11.87 & 0.63 & 0.62 \\
\hline S4928 & $\mathrm{O}=\mathrm{c} 1 \mathrm{sc} 2 \mathrm{c}([\mathrm{nH}] 1) \mathrm{ncnc} 2$ & 1.69 & 0.98 & 5.12 & 6.50 & 10.20 & 12.35 & 0.49 & 0.49 \\
\hline S494 & $\mathrm{O}=\mathrm{c} 1 \mathrm{cnsc} 2 \mathrm{n} 1 \mathrm{ccc} 2$ & -0.42 & -0.10 & 2.52 & 5.84 & 7.90 & 13.60 & 0.51 & 0.55 \\
\hline S497 & $\mathrm{O}=\mathrm{c} 1 \mathrm{ncsc} 2 \mathrm{c} 1 \mathrm{cc}[\mathrm{nH}] 2$ & -1.53 & -0.85 & 9.76 & 8.87 & 23.82 & 22.54 & 0.52 & 0.56 \\
\hline S50 & c1ccnnc1 & -0.74 & -1.19 & 5.49 & 4.99 & 15.44 & 14.77 & 0.43 & 0.45 \\
\hline $\mathbf{S 5 0 0}$ & $\mathrm{O}=\mathrm{c} 1 \mathrm{nn}[\mathrm{nH}] \mathrm{c} 2 \mathrm{c} 1[\mathrm{nH}] \mathrm{cc} 2$ & -0.08 & 0.07 & 7.91 & 10.86 & 21.71 & 23.88 & 0.56 & 0.50 \\
\hline S501 & $\mathrm{O}=\mathrm{c} 1 \mathrm{nn}[\mathrm{nH}] \mathrm{c} 2 \mathrm{c} 1 \mathrm{cc}[\mathrm{nH}] 2$ & -0.30 & 0.07 & 14.45 & 15.41 & 29.98 & 30.82 & 0.42 & 0.44 \\
\hline S51 & $\mathrm{O}=\mathrm{c} 1 \mathrm{cccc}[\mathrm{nH}] 1$ & -1.77 & -2.25 & 5.75 & 4.24 & 17.36 & 14.82 & 0.51 & 0.56 \\
\hline S516 & $\mathrm{O}=\mathrm{c} 1 \mathrm{oc} 2 \operatorname{ccc} 2 \mathrm{c}(=\mathrm{O})[\mathrm{nH}] 1$ & 1.56 & 1.36 & 6.97 & 7.22 & 11.97 & 14.21 & 0.50 & 0.46 \\
\hline S5176 & $\mathrm{O}=\mathrm{c} 1[\mathrm{nH}] \mathrm{nc} 2 \mathrm{n} 1 \mathrm{cccc} 2$ & -0.92 & -1.01 & 4.25 & 5.25 & 13.03 & 13.43 & 0.41 & 0.44 \\
\hline S5177 & $\mathrm{O}=\mathrm{c} 1[\mathrm{nH}] \mathrm{nc} 2 \mathrm{n} 1 \mathrm{ccnc} 2$ & 1.03 & 0.82 & 4.08 & 7.54 & 10.29 & 14.42 & 0.52 & 0.47 \\
\hline S5178 & $\mathrm{O}=\mathrm{c} 1[\mathrm{nH}] \mathrm{nc} 2 \mathrm{n} 1 \mathrm{cncc} 2$ & 1.03 & 0.82 & 4.15 & 7.40 & 10.48 & 14.74 & 0.47 & 0.54 \\
\hline S5179 & $\mathrm{O}=\mathrm{c} 1[\mathrm{nH}] \mathrm{nc} 2 \mathrm{n} 1 \mathrm{nccc} 2$ & 0.23 & 0.82 & 7.36 & 6.82 & 19.12 & 15.09 & 0.61 & 0.57 \\
\hline S5197 & $\mathrm{O}=\mathrm{c} 1[\mathrm{nH}] \mathrm{nc} 2 \mathrm{n} 1 \mathrm{ncnc} 2$ & 2.19 & 2.65 & 7.09 & 8.52 & 16.09 & 14.79 & 0.58 & 0.49 \\
\hline S52 & $\mathrm{O}=\mathrm{c} 1 \mathrm{ccc} \operatorname{co} 1$ & -0.31 & -0.65 & 5.51 & 4.94 & 16.04 & 14.56 & 0.41 & 0.48 \\
\hline S5231 & $\mathrm{O}=\mathrm{c} 1[\mathrm{nH}] \mathrm{nc} 2 \mathrm{n} 1 \mathrm{nccn} 2$ & 1.91 & 2.65 & 9.39 & 7.93 & 18.42 & 13.89 & 0.40 & 0.42 \\
\hline S54 & c1cencn 1 & -0.83 & -1.19 & 4.15 & 3.38 & 8.84 & 11.22 & 0.44 & 0.50 \\
\hline S55 & c1cnnnc1 & 1.23 & 0.64 & 8.49 & 7.04 & 17.64 & 16.37 & 0.26 & 0.32 \\
\hline S5528 & $\mathrm{C} 1 \mathrm{C}=[\mathrm{S}] \mathrm{c} 2 \mathrm{n} 1 \mathrm{c}(=\mathrm{O}) \mathrm{sn} 2$ & 0.50 & 0.43 & 6.83 & 6.13 & 13.21 & 11.94 & 0.66 & 0.60 \\
\hline S554 & {$[\mathrm{nH}] 1 \mathrm{cc} 2 \mathrm{c}(\mathrm{c} 1) \mathrm{cco} 2$} & -5.81 & -4.97 & 3.32 & 3.30 & 13.99 & 14.00 & 0.61 & 0.52 \\
\hline S555 & c1cc $2 \mathrm{c}(\mathrm{o} 1) \mathrm{occ} 2$ & -3.48 & -3.37 & 0.03 & 2.33 & 5.81 & 10.70 & 0.49 & 0.57 \\
\hline S557 & $\mathrm{o} 1 \mathrm{cc} 2 \mathrm{c}(\mathrm{c} 1) \mathrm{cco} 2$ & -3.61 & -3.37 & 0.97 & 2.15 & 7.30 & 10.62 & 0.61 & 0.65 \\
\hline S558 & $\mathrm{c} 1 \mathrm{oc} 2 \mathrm{c}(\mathrm{c} 1) \operatorname{ccs} 2$ & -4.15 & -4.06 & 0.16 & 1.54 & 7.23 & 10.03 & 0.36 & 0.39 \\
\hline S559 & $\mathrm{c} 1 \mathrm{cc} 2 \mathrm{c}(\mathrm{o} 1) \operatorname{ccs} 2$ & -4.16 & -4.06 & 0.25 & 1.35 & 7.40 & 9.99 & 0.48 & 0.50 \\
\hline
\end{tabular}




\begin{tabular}{|c|c|c|c|c|c|c|c|c|c|}
\hline S56 & $\mathrm{O}=\mathrm{c} 1 \mathrm{ccc} n[\mathrm{nH}] 1$ & -0.03 & -0.42 & 5.66 & 4.07 & 14.84 & 11.88 & 0.60 & 0.54 \\
\hline S560 & $\mathrm{s} 1 \mathrm{cc} 2 \mathrm{c}(\mathrm{c} 1) \mathrm{cco} 2$ & -4.13 & -4.06 & 0.79 & 1.41 & 8.08 & 10.00 & 0.46 & 0.54 \\
\hline S561 & c1cc2c(o1)[nH]nc2 & -3.30 & -3.14 & 2.34 & 3.62 & 10.55 & 13.13 & 0.46 & 0.49 \\
\hline S562 & $\mathrm{c} 1 \mathrm{oc} 2 \mathrm{c}(\mathrm{c} 1)[\mathrm{nH}] \mathrm{nc} 2$ & -3.32 & -3.14 & 2.92 & 4.37 & 11.31 & 13.64 & 0.47 & 0.54 \\
\hline S563 & $\mathrm{c} 1 \mathrm{cc} 2 \mathrm{c}(\mathrm{o} 1) \mathrm{n}[\mathrm{nH}] \mathrm{c} 2$ & -3.80 & -3.14 & 3.57 & 4.56 & 13.04 & 13.90 & 0.40 & 0.46 \\
\hline S564 & $\mathrm{c} 1 \mathrm{cc} 2 \mathrm{c}(\mathrm{o} 1) \mathrm{c}[\mathrm{nH}] \mathrm{n} 2$ & -3.98 & -3.14 & 3.28 & 3.89 & 12.07 & 13.25 & 0.41 & 0.45 \\
\hline S565 & c1cc2c(o1)onc2 & -1.07 & -1.54 & 3.81 & 4.10 & 11.71 & 11.50 & 0.31 & 0.38 \\
\hline S567 & c1cc2c(o1)noc2 & -1.25 & -1.54 & 4.32 & 4.37 & 13.52 & 11.86 & 0.37 & 0.43 \\
\hline S5673 & $\mathrm{c} 1[\mathrm{nH}] \mathrm{n} 2 \mathrm{c}(\mathrm{c} 1) \mathrm{nnc} 2$ & -1.78 & -2.16 & 11.60 & 9.90 & 27.86 & 22.00 & 0.72 & 0.66 \\
\hline S5674 & c1cn2c([nH]1)nnc2 & -1.72 & -2.16 & 7.57 & 7.14 & 22.74 & 18.24 & 0.41 & 0.46 \\
\hline S5678 & $\mathrm{c} 1 \mathrm{cn} 2 \mathrm{c}(\mathrm{s} 1) \mathrm{nnc} 2$ & -0.46 & -1.25 & 8.24 & 6.96 & 21.17 & 16.05 & 0.66 & 0.60 \\
\hline S5679 & $\mathrm{n} 1 \mathrm{cn} 2 \mathrm{c}(\mathrm{n} 1) \mathrm{cn}[\mathrm{nH}] 2$ & 0.12 & -0.33 & 11.19 & 10.37 & 23.45 & 20.19 & 0.60 & 0.56 \\
\hline S5680 & $\mathrm{n} 1 \mathrm{nc} 2 \mathrm{n}(\mathrm{c} 1) \mathrm{cn}[\mathrm{nH}] 2$ & 0.28 & -0.33 & 7.67 & 7.61 & 19.29 & 16.43 & 0.50 & 0.57 \\
\hline S5684 & $\mathrm{n} 1 \mathrm{nc} 2 \mathrm{n}(\mathrm{c} 1) \mathrm{cns} 2$ & 1.53 & 0.58 & 10.24 & 8.33 & 19.87 & 15.41 & 0.58 & 0.53 \\
\hline S5685 & $\mathrm{c} 1[\mathrm{nH}] \mathrm{n} 2 \mathrm{c}(\mathrm{n} 1) \mathrm{nnc} 2$ & 0.25 & -0.33 & 14.95 & 13.78 & 30.41 & 26.47 & 0.60 & 0.65 \\
\hline S5686 & $\mathrm{c} 1 \mathrm{nn} 2 \mathrm{c}([\mathrm{nH}] 1) \mathrm{nnc} 2$ & -0.15 & -0.33 & 10.90 & 8.55 & 23.79 & 17.93 & 0.53 & 0.47 \\
\hline S5687 & c1on2c(n1)nnc2 & 1.71 & 1.27 & 10.35 & 9.19 & 21.89 & 18.39 & 0.65 & 0.73 \\
\hline S5688 & c1nn2c(o1)nnc2 & 1.78 & 1.27 & 9.57 & 7.69 & 20.00 & 14.45 & 0.33 & 0.34 \\
\hline S569 & $\mathrm{c} 1 \mathrm{cc} 2 \mathrm{c}(\mathrm{o} 1) \mathrm{snc} 2$ & -2.02 & -2.23 & 3.04 & 3.25 & 10.25 & 10.66 & 0.37 & 0.41 \\
\hline S5690 & c1nn2c(s1)nnc2 & 0.84 & 0.58 & 8.59 & 6.93 & 19.53 & 13.84 & 0.42 & 0.45 \\
\hline S57 & $\mathrm{O}=\mathrm{c} 1 \mathrm{nccc}[\mathrm{nH}] 1$ & 0.09 & -0.42 & 9.33 & 7.65 & 22.41 & 19.64 & 0.50 & 0.53 \\
\hline S5703 & $\operatorname{c} 1 \mathrm{ccc} 2 \mathrm{n}(\mathrm{c} 1) \operatorname{cnn} 2$ & -0.82 & -1.78 & 7.00 & 6.66 & 20.29 & 16.85 & 0.99 & 0.94 \\
\hline S5704 & $\mathrm{n} 1 \mathrm{ccn} 2 \mathrm{c}(\mathrm{c} 1) \mathrm{nnc} 2$ & 1.09 & 0.05 & 9.10 & 8.37 & 18.95 & 17.31 & 0.54 & 0.48 \\
\hline S5705 & $\mathrm{c} 1 \mathrm{ncn} 2 \mathrm{c}(\mathrm{c} 1) \mathrm{nnc} 2$ & 1.04 & 0.05 & 8.18 & 7.50 & 18.25 & 15.57 & 0.41 & 0.44 \\
\hline S5706 & c1cnn2c(c1)nnc2 & 0.39 & 0.05 & 7.55 & 7.05 & 19.05 & 15.20 & 0.45 & 0.47 \\
\hline S5707 & $\mathrm{c} 1 \mathrm{ccn} 2 \mathrm{c}(\mathrm{n} 1) \mathrm{nnc} 2$ & 1.00 & 0.05 & 10.65 & 9.62 & 24.42 & 20.23 & 0.51 & 0.44 \\
\hline S5708 & $\mathrm{n} 1 \mathrm{ccn} 2 \mathrm{c}(\mathrm{n} 1) \mathrm{nnc} 2$ & 2.92 & 1.88 & 13.33 & 12.36 & 23.82 & 22.93 & 0.73 & 0.68 \\
\hline S571 & c1cc2c(o1)nsc2 & -2.14 & -2.23 & 3.06 & 3.56 & 11.34 & 11.07 & 0.62 & 0.54 \\
\hline S5711 & $\mathrm{O}=\mathrm{c} 1[\mathrm{nH}] \mathrm{ccn} 2 \mathrm{c} 1 \mathrm{nnc} 2$ & 1.19 & 0.82 & 11.03 & 11.55 & 24.61 & 24.43 & 0.77 & 0.75 \\
\hline S5712 & $\mathrm{O}=\mathrm{c} 1[\mathrm{nH}] \operatorname{ccc} 2 \mathrm{n} 1 \mathrm{cnn} 2$ & 1.33 & 0.82 & 11.09 & 9.69 & 22.29 & 18.75 & 0.26 & 0.32 \\
\hline S5713 & $\mathrm{O}=\mathrm{c} 1 \mathrm{ccc} 2 \mathrm{n}([\mathrm{nH}] 1) \operatorname{cnn} 2$ & 2.36 & 0.82 & 10.09 & 10.70 & 18.40 & 19.56 & 0.50 & 0.49 \\
\hline
\end{tabular}




\begin{tabular}{|c|c|c|c|c|c|c|c|c|c|}
\hline S5714 & $\mathrm{O}=\mathrm{c} 1 \mathrm{ccn} 2 \mathrm{c}([\mathrm{nH}] 1) \mathrm{nnc} 2$ & 2.40 & 0.82 & 11.10 & 8.76 & 19.74 & 18.03 & 0.59 & 0.63 \\
\hline S5723 & $\mathrm{c} 1 \mathrm{ncn} 2 \mathrm{c}(\mathrm{n} 1) \mathrm{nnc} 2$ & 2.88 & 1.88 & 12.10 & 9.88 & 22.52 & 17.65 & 0.88 & 0.80 \\
\hline S5724 & $\mathrm{n} 1 \mathrm{cnn} 2 \mathrm{c}(\mathrm{c} 1) \mathrm{nnc} 2$ & 2.32 & 1.88 & 6.71 & 8.17 & 14.52 & 14.36 & 0.64 & 0.60 \\
\hline S5725 & $\mathrm{n} 1 \mathrm{ncn} 2 \mathrm{c}(\mathrm{n} 1) \mathrm{nnc} 2$ & 4.96 & 3.71 & 15.37 & 13.64 & 22.05 & 22.60 & 0.57 & 0.67 \\
\hline S5727 & $\mathrm{O}=\mathrm{c} 1[\mathrm{nH}] \mathrm{ncn} 2 \mathrm{c} 1 \mathrm{nnc} 2$ & 3.02 & 2.65 & 12.24 & 10.60 & 22.21 & 19.56 & 0.28 & 0.34 \\
\hline S5728 & $\mathrm{O}=\mathrm{c} 1[\mathrm{nH}] \mathrm{ncc} 2 \mathrm{n} 1 \mathrm{cnn} 2$ & 3.16 & 2.65 & 8.89 & 9.61 & 16.06 & 15.62 & 0.32 & 0.37 \\
\hline S5729 & $\mathrm{O}=\mathrm{c} 1 \mathrm{ncn} 2 \mathrm{c}([\mathrm{nH}] 1) \mathrm{nnc} 2$ & 4.15 & 2.65 & 13.07 & 11.39 & 19.62 & 20.91 & 0.83 & 0.77 \\
\hline S573 & $\mathrm{c} 1 \mathrm{cc} 2 \mathrm{c}(\mathrm{o} 1)[\mathrm{nH}] \mathrm{cn} 2$ & -3.54 & -3.14 & 6.24 & 5.25 & 16.78 & 15.77 & 0.58 & 0.57 \\
\hline S5730 & $\mathrm{O}=\mathrm{c} 1 \mathrm{ncc} 2 \mathrm{n}([\mathrm{nH}] 1) \mathrm{cnn} 2$ & 4.15 & 2.65 & 12.99 & 14.19 & 19.17 & 24.18 & 0.46 & 0.50 \\
\hline S5739 & $\mathrm{O}=\mathrm{c} 1 \mathrm{cnc} 2 \mathrm{n}([\mathrm{nH}] 1) \mathrm{cnn} 2$ & 4.12 & 2.65 & 13.53 & 14.16 & 22.66 & 22.89 & 0.42 & 0.36 \\
\hline S574 & $\mathrm{c} 1 \mathrm{oc} 2 \mathrm{c}(\mathrm{c} 1)[\mathrm{nH}] \mathrm{cn} 2$ & -3.38 & -3.14 & 6.62 & 6.66 & 17.55 & 16.94 & 0.45 & 0.51 \\
\hline S5740 & $\mathrm{O}=\mathrm{c} 1 \mathrm{cnn} 2 \mathrm{c}([\mathrm{nH}] 1) \mathrm{nnc} 2$ & 3.62 & 2.65 & 9.95 & 9.65 & 17.49 & 16.33 & 0.67 & 0.61 \\
\hline S5754 & $\mathrm{O}=\mathrm{c} 1 \mathrm{cc}[\mathrm{nH}] \mathrm{c} 2 \mathrm{n} 1 \mathrm{cnn} 2$ & 1.30 & 0.82 & 11.72 & 9.82 & 21.82 & 20.04 & 0.35 & 0.43 \\
\hline S5755 & $\mathrm{O}=\mathrm{c} 1 \mathrm{cc}[\mathrm{nH}] \mathrm{n} 2 \mathrm{c} 1 \mathrm{nnn} 2$ & 1.16 & 0.82 & 16.35 & 15.54 & 30.74 & 31.05 & 0.41 & 0.44 \\
\hline S5757 & $\mathrm{O}=\mathrm{c} 1 \mathrm{nc}[\mathrm{nH}] \mathrm{n} 2 \mathrm{c} 1 \mathrm{nnc} 2$ & 3.02 & 2.65 & 18.82 & 19.03 & 31.94 & 35.68 & 0.33 & 0.38 \\
\hline S5758 & $\mathrm{c} 1 \mathrm{cnc} 2 \mathrm{n}(\mathrm{n} 1) \mathrm{cnn} 2$ & 2.15 & 1.88 & 10.27 & 8.99 & 21.53 & 16.34 & 0.49 & 0.54 \\
\hline S576 & $\mathrm{c} 1 \mathrm{oc} 2 \mathrm{c}(\mathrm{c} 1) \mathrm{ocn} 2$ & -1.58 & -1.54 & 2.17 & 3.63 & 8.70 & 11.18 & 0.62 & 0.54 \\
\hline S5760 & $\mathrm{n} 1 \mathrm{cnn} 2 \mathrm{c}(\mathrm{n} 1) \mathrm{nnc} 2$ & 4.08 & 3.71 & 10.42 & 11.14 & 17.80 & 17.74 & 0.38 & 0.44 \\
\hline S5761 & $\mathrm{O}=\mathrm{c} 1[\mathrm{nH}] \mathrm{cnc} 2 \mathrm{n} 1 \mathrm{cnn} 2$ & 3.15 & 2.65 & 13.96 & 12.19 & 25.10 & 20.01 & 0.64 & 0.67 \\
\hline S5762 & $\mathrm{O}=\mathrm{c} 1[\mathrm{nH}] \mathrm{cnn} 2 \mathrm{c} 1 \mathrm{nnc} 2$ & 2.50 & 2.65 & 13.40 & 11.47 & 24.80 & 20.66 & 0.93 & 0.88 \\
\hline S577 & $\operatorname{c} 1 \mathrm{cc} 2 \mathrm{c}(\mathrm{o} 1) \operatorname{scn} 2$ & -2.53 & -2.23 & 1.44 & 2.54 & 7.93 & 10.24 & 0.66 & 0.59 \\
\hline S578 & $\mathrm{c} 1 \mathrm{oc} 2 \mathrm{c}(\mathrm{c} 1) \operatorname{sen} 2$ & -2.38 & -2.23 & 1.99 & 3.01 & 9.15 & 10.85 & 0.52 & 0.60 \\
\hline S5787 & $\mathrm{O}=\mathrm{c} 1 \operatorname{cssc} 2 \mathrm{n} 1 \mathrm{cnn} 2$ & 2.16 & 1.73 & 9.45 & 8.39 & 18.62 & 15.76 & 0.54 & 0.59 \\
\hline S5788 & $\mathrm{O}=\mathrm{c} 1 \mathrm{cn}[\mathrm{nH}] \mathrm{c} 2 \mathrm{n} 1 \mathrm{cnn} 2$ & 3.10 & 2.65 & 8.92 & 9.75 & 16.23 & 16.90 & 0.33 & 0.36 \\
\hline S5792 & $\mathrm{O}=\mathrm{c} 1 \mathrm{cnsc} 2 \mathrm{n} 1 \mathrm{cnn} 2$ & 3.96 & 3.56 & 10.71 & 9.76 & 17.41 & 14.53 & 0.39 & 0.38 \\
\hline S581 & {$[\mathrm{nH}] 1 \mathrm{nc} 2 \mathrm{c}(\mathrm{n} 1) \mathrm{cco} 2$} & -1.93 & -1.31 & 2.44 & 4.13 & 7.62 & 10.89 & 0.34 & 0.39 \\
\hline S5823 & $\mathrm{c} 1 \mathrm{nn} 2 \mathrm{c}(\mathrm{n} 1) \mathrm{cc}[\mathrm{nH}] 2$ & -2.50 & -2.16 & 8.10 & 6.22 & 19.09 & 15.38 & 0.53 & 0.57 \\
\hline S5824 & $\mathrm{c} 1 \mathrm{cn} 2 \mathrm{c}([\mathrm{nH}] 1) \mathrm{ncn} 2$ & -2.42 & -2.16 & 6.79 & 5.37 & 15.98 & 14.57 & 0.70 & 0.64 \\
\hline S5826 & $\mathrm{c} 1 \mathrm{cn} 2 \mathrm{c}(\mathrm{o} 1) \mathrm{ncn} 2$ & -0.50 & -0.56 & 6.28 & 5.18 & 13.73 & 12.03 & 0.73 & 0.76 \\
\hline S5828 & $\mathrm{c} 1 \mathrm{cn} 2 \mathrm{c}(\mathrm{s} 1) \mathrm{ncn} 2$ & -1.31 & -1.25 & 5.40 & 4.38 & 12.73 & 11.31 & 0.60 & 0.50 \\
\hline S5829 & $\mathrm{c} 1 \mathrm{nn} 2 \mathrm{c}(\mathrm{n} 1) \mathrm{cn}[\mathrm{nH}] 2$ & -0.52 & -0.33 & 6.81 & 7.12 & 13.77 & 14.50 & 0.32 & 0.36 \\
\hline
\end{tabular}




\begin{tabular}{|c|c|c|c|c|c|c|c|c|c|}
\hline S5830 & $\mathrm{c} 1 \mathrm{nn} 2 \mathrm{c}(\mathrm{n} 1)[\mathrm{nH}] \mathrm{nc} 2$ & -0.37 & -0.33 & 5.10 & 6.26 & 10.18 & 13.69 & 0.24 & 0.29 \\
\hline S5832 & c1nn2c(n1)onc2 & 1.84 & 1.27 & 7.46 & 7.03 & 11.76 & 12.49 & 0.56 & 0.52 \\
\hline S5834 & $\mathrm{c} 1 \mathrm{nn} 2 \mathrm{c}(\mathrm{n} 1) \mathrm{snc} 2$ & 0.74 & 0.58 & 4.63 & 6.17 & 8.71 & 11.61 & 0.54 & 0.57 \\
\hline S5835 & $\mathrm{c} 1 \mathrm{nc} 2 \mathrm{n}(\mathrm{n} 1)[\mathrm{nH}] \mathrm{cn} 2$ & -0.48 & -0.33 & 11.66 & 9.52 & 22.53 & 18.58 & 0.88 & 0.86 \\
\hline S5836 & $\mathrm{c} 1 \mathrm{nc} 2 \mathrm{n}(\mathrm{n} 1) \mathrm{nc}[\mathrm{nH}] 2$ & -0.60 & -0.33 & 10.78 & 7.90 & 20.24 & 16.74 & 0.63 & 0.58 \\
\hline S5838 & $\operatorname{c} 1 n c 2 n(n 1) n c o 2$ & 1.31 & 1.27 & 8.36 & 6.17 & 13.73 & 12.04 & 1.05 & 1.08 \\
\hline S5840 & $\mathrm{c} 1 \mathrm{nc} 2 \mathrm{n}(\mathrm{n} 1) \mathrm{ncs} 2$ & 0.27 & 0.58 & 7.33 & 5.47 & 13.72 & 11.59 & 0.64 & 0.58 \\
\hline S5853 & $\mathrm{c} 1 \mathrm{ccc} 2 \mathrm{n}(\mathrm{c} 1) \mathrm{ncn} 2$ & -1.74 & -1.78 & 4.24 & 4.24 & 12.17 & 12.19 & 0.58 & 0.53 \\
\hline S5854 & $\mathrm{n} 1 \mathrm{ccn} 2 \mathrm{c}(\mathrm{c} 1) \mathrm{ncn} 2$ & 0.20 & 0.05 & 4.54 & 5.98 & 9.30 & 12.70 & 0.31 & 0.37 \\
\hline S5855 & $\mathrm{c} 1 \mathrm{ncn} 2 \mathrm{c}(\mathrm{c} 1) \mathrm{ncn} 2$ & 0.20 & 0.05 & 3.01 & 5.78 & 7.58 & 12.43 & 0.32 & 0.35 \\
\hline S5856 & $\mathrm{c} 1 \mathrm{cnn} 2 \mathrm{c}(\mathrm{c} 1) \mathrm{ncn} 2$ & -0.24 & 0.05 & 6.66 & 5.70 & 14.36 & 12.90 & 0.33 & 0.35 \\
\hline S5857 & $\mathrm{c} 1 \mathrm{ccn} 2 \mathrm{c}(\mathrm{n} 1) \mathrm{ncn} 2$ & 0.03 & 0.05 & 7.08 & 6.45 & 16.60 & 13.90 & 0.70 & 0.73 \\
\hline S5858 & $\mathrm{n} 1 \mathrm{ccn} 2 \mathrm{c}(\mathrm{n} 1) \mathrm{ncn} 2$ & 2.01 & 1.88 & 8.99 & 9.21 & 16.08 & 16.66 & 0.61 & 0.52 \\
\hline S5859 & $\mathrm{c} 1 \mathrm{nnn} 2 \mathrm{c}(\mathrm{c} 1) \mathrm{ncn} 2$ & 1.67 & 1.88 & 7.16 & 8.27 & 12.32 & 15.39 & 0.29 & 0.31 \\
\hline S5860 & $\mathrm{n} 1 \mathrm{ncc} 2 \mathrm{n}(\mathrm{c} 1) \mathrm{ncn} 2$ & 2.35 & 1.88 & 6.46 & 8.54 & 11.10 & 15.19 & 0.65 & 0.72 \\
\hline S5861 & $\mathrm{O}=\mathrm{c} 1[\mathrm{nH}] \mathrm{ccn} 2 \mathrm{c} 1 \mathrm{ncn} 2$ & 0.32 & 0.82 & 9.63 & 8.27 & 20.09 & 17.39 & 0.60 & 0.59 \\
\hline S5862 & $\mathrm{O}=\mathrm{c} 1[\mathrm{nH}] \operatorname{ccc} 2 \mathrm{n} 1 \mathrm{ncn} 2$ & 0.29 & 0.82 & 10.22 & 7.67 & 19.52 & 16.20 & 0.44 & 0.40 \\
\hline S5864 & $\mathrm{O}=\mathrm{c} 1 \mathrm{ccn} 2 \mathrm{c}([\mathrm{nH}] 1) \mathrm{ncn} 2$ & 1.48 & 0.82 & 5.59 & 7.22 & 11.70 & 14.75 & 0.31 & 0.38 \\
\hline S5873 & $\mathrm{c} 1 \mathrm{ncn} 2 \mathrm{c}(\mathrm{n} 1) \mathrm{ncn} 2$ & 1.98 & 1.88 & 7.05 & 7.40 & 13.10 & 12.85 & 0.67 & 0.57 \\
\hline S5874 & $\mathrm{n} 1 \mathrm{cnn} 2 \mathrm{c}(\mathrm{c} 1) \mathrm{ncn} 2$ & 1.71 & 1.88 & 5.54 & 6.85 & 9.68 & 12.12 & 0.64 & 0.60 \\
\hline S5875 & $\mathrm{n} 1 \mathrm{ncn} 2 \mathrm{c}(\mathrm{n} 1) \mathrm{ncn} 2$ & 4.12 & 3.71 & 10.46 & 11.18 & 15.03 & 17.86 & 0.45 & 0.43 \\
\hline S5876 & $\mathrm{n} 1 \mathrm{nnn} 2 \mathrm{c}(\mathrm{c} 1) \mathrm{ncn} 2$ & 3.80 & 3.71 & 8.94 & 10.44 & 12.55 & 16.85 & 0.51 & 0.45 \\
\hline S5877 & $\mathrm{O}=\mathrm{c} 1[\mathrm{nH}] \mathrm{ncn} 2 \mathrm{c} 1 \mathrm{ncn} 2$ & 2.22 & 2.65 & 7.54 & 8.01 & 15.52 & 14.05 & 0.61 & 0.64 \\
\hline S5878 & $\mathrm{O}=\mathrm{c} 1[\mathrm{nH}] \mathrm{ncc} 2 \mathrm{n} 1 \mathrm{ncn} 2$ & 2.12 & 2.65 & 7.09 & 7.62 & 13.84 & 13.12 & 0.51 & 0.45 \\
\hline S5879 & $\mathrm{O}=\mathrm{c} 1 \mathrm{ncn} 2 \mathrm{c}([\mathrm{nH}] 1) \mathrm{ncn} 2$ & 3.33 & 2.65 & 8.87 & 10.55 & 16.28 & 19.17 & 0.45 & 0.43 \\
\hline S5890 & $\mathrm{O}=\mathrm{c} 1 \mathrm{cnn} 2 \mathrm{c}([\mathrm{nH}] 1) \mathrm{ncn} 2$ & 2.99 & 2.65 & 8.62 & 9.18 & 12.33 & 15.42 & 0.68 & 0.54 \\
\hline S5904 & $\mathrm{O}=\mathrm{c} 1 \mathrm{cc}[\mathrm{nH}] \mathrm{c} 2 \mathrm{n} 1 \mathrm{ncn} 2$ & 0.28 & 0.82 & 11.38 & 9.43 & 23.10 & 19.99 & 0.62 & 0.70 \\
\hline S5906 & $\mathrm{O}=\mathrm{c} 1 \mathrm{nc}[\mathrm{nH}] \mathrm{c} 2 \mathrm{n} 1 \mathrm{ncn} 2$ & 2.12 & 2.65 & 13.81 & 12.76 & 25.04 & 24.41 & 0.45 & 0.40 \\
\hline S5908 & $\mathrm{c} 1 \mathrm{cnc} 2 \mathrm{n}(\mathrm{n} 1) \mathrm{ncn} 2$ & 1.46 & 1.88 & 8.43 & 6.89 & 14.79 & 12.37 & 0.60 & 0.53 \\
\hline S5909 & $\mathrm{c} 1 \mathrm{nnn} 2 \mathrm{c}(\mathrm{n} 1) \mathrm{ncn} 2$ & 3.43 & 3.71 & 8.36 & 8.87 & 10.77 & 13.56 & 0.43 & 0.48 \\
\hline S5910 & $\mathrm{n} 1 \mathrm{cnn} 2 \mathrm{c}(\mathrm{n} 1) \mathrm{ncn} 2$ & 3.47 & 3.71 & 8.45 & 9.07 & 11.60 & 13.83 & 0.37 & 0.43 \\
\hline
\end{tabular}




\begin{tabular}{|c|c|c|c|c|c|c|c|c|c|}
\hline S5911 & $\mathrm{O}=\mathrm{c} 1[\mathrm{nH}] \mathrm{cnc} 2 \mathrm{n} 1 \mathrm{ncn} 2$ & 2.05 & 2.65 & 12.73 & 9.42 & 19.17 & 15.79 & 0.47 & 0.53 \\
\hline S5912 & $\mathrm{O}=\mathrm{c} 1[\mathrm{nH}] \mathrm{cnn} 2 \mathrm{c} 1 \mathrm{ncn} 2$ & 1.89 & 2.65 & 12.16 & 9.27 & 19.31 & 15.98 & 0.64 & 0.58 \\
\hline S5919 & $\mathrm{O}=\mathrm{c} 1 \mathrm{scnc} 2 \mathrm{n} 1 \mathrm{ncn} 2$ & 2.58 & 3.56 & 9.01 & 8.31 & 14.19 & 13.09 & 0.62 & 0.54 \\
\hline S5937 & $\mathrm{O}=\mathrm{c} 1 \mathrm{ccsc} 2 \mathrm{n} 1 \mathrm{ncn} 2$ & 1.01 & 1.73 & 8.49 & 6.93 & 17.13 & 14.32 & 0.39 & 0.44 \\
\hline S5938 & $\mathrm{O}=\mathrm{c} 1 \mathrm{cn}[\mathrm{nH}] \mathrm{c} 2 \mathrm{n} 1 \mathrm{ncn} 2$ & 2.10 & 2.65 & 8.24 & 9.38 & 16.16 & 16.92 & 0.81 & 0.79 \\
\hline S594 & $\operatorname{c} 1 \mathrm{ccc} 2 \mathrm{c}(\mathrm{c} 1) \operatorname{occ} 2$ & -4.55 & -4.59 & 0.37 & 1.46 & 8.80 & 10.93 & 0.67 & 0.63 \\
\hline S5942 & $\mathrm{O}=\mathrm{c} 1 \mathrm{cnsc} 2 \mathrm{n} 1 \mathrm{ncn} 2$ & 2.84 & 3.56 & 8.49 & 8.33 & 13.87 & 13.14 & 0.27 & 0.38 \\
\hline S595 & c1ncc2c(c1)occ2 & -2.55 & -2.76 & 2.95 & 2.88 & 11.15 & 11.38 & 0.31 & 0.37 \\
\hline S596 & $\mathrm{c} 1 \mathrm{ncc} 2 \mathrm{c}(\mathrm{c} 1) \mathrm{cco} 2$ & -2.53 & -2.76 & 3.27 & 3.14 & 11.71 & 11.64 & 0.54 & 0.62 \\
\hline S597 & $\mathrm{c} 1 \mathrm{cnc} 2 \mathrm{c}(\mathrm{c} 1) \mathrm{cco} 2$ & -2.83 & -2.76 & 2.29 & 3.50 & 11.05 & 11.99 & 0.69 & 0.67 \\
\hline S5974 & $\mathrm{c} 1 \mathrm{cn} 2 \mathrm{c}([\mathrm{nH}] 1) \mathrm{nnn} 2$ & 0.11 & -0.33 & 10.41 & 8.99 & 23.55 & 19.05 & 0.51 & 0.45 \\
\hline S5976 & $\mathrm{c} 1 \mathrm{cn} 2 \mathrm{c}(\mathrm{o} 1) \mathrm{nnn} 2$ & 2.04 & 1.27 & 10.23 & 8.44 & 20.68 & 16.00 & 0.54 & 0.60 \\
\hline S5978 & $\mathrm{c} 1 \mathrm{cn} 2 \mathrm{c}(\mathrm{s} 1) \mathrm{nnn} 2$ & 1.19 & 0.58 & 9.13 & 7.66 & 19.78 & 15.34 & 0.33 & 0.40 \\
\hline S598 & $\mathrm{c} 1 \mathrm{cnc} 2 \mathrm{c}(\mathrm{c} 1) \mathrm{occ} 2$ & -3.01 & -2.76 & 1.68 & 2.78 & 9.78 & 11.29 & 0.42 & 0.50 \\
\hline S60 & $\mathrm{O}=\mathrm{c} 1 \mathrm{ccc} \cos 1$ & 0.84 & 0.49 & 7.41 & 5.35 & 15.00 & 12.52 & 0.41 & 0.40 \\
\hline S600 & $\mathrm{c} 1 \mathrm{nnn} 2 \mathrm{c}(\mathrm{c} 1) \operatorname{cco} 2$ & -0.66 & -0.93 & 5.70 & 6.21 & 16.50 & 14.95 & 0.32 & 0.42 \\
\hline S601 & $\mathrm{n} 1 \mathrm{ncc} 2 \mathrm{c}(\mathrm{c} 1) \operatorname{occ} 2$ & -0.33 & -0.93 & 6.23 & 5.58 & 17.15 & 14.34 & 0.31 & 0.34 \\
\hline S6018 & $\operatorname{c1ccc} 2 \mathrm{n}(\mathrm{c} 1) \mathrm{nnn} 2$ & 0.80 & 0.05 & 7.86 & 7.59 & 19.20 & 16.25 & 0.44 & 0.49 \\
\hline S6019 & $\mathrm{n} 1 \mathrm{ccn} 2 \mathrm{c}(\mathrm{c} 1) \mathrm{nnn} 2$ & 2.69 & 1.88 & 8.15 & 8.80 & 16.16 & 15.58 & 0.75 & 0.80 \\
\hline S602 & $\mathrm{O}=\mathrm{c} 1[\mathrm{nH}] \mathrm{ccc} 2 \mathrm{c} 1 \mathrm{cco} 2$ & -2.33 & -1.99 & 5.38 & 4.87 & 16.36 & 14.28 & 0.69 & 0.61 \\
\hline S6020 & c1ncn2c(c1)nnn2 & 2.67 & 1.88 & 7.35 & 8.60 & 15.44 & 15.31 & 0.67 & 0.62 \\
\hline S6021 & $\mathrm{c} 1 \mathrm{cnn} 2 \mathrm{c}(\mathrm{c} 1) \mathrm{nnn} 2$ & 2.36 & 1.88 & 10.66 & 9.06 & 20.92 & 16.96 & 0.58 & 0.53 \\
\hline S6022 & $\operatorname{c} 1 \mathrm{ccn} 2 \mathrm{c}(\mathrm{n} 1) \mathrm{nnn} 2$ & 2.62 & 1.88 & 11.01 & 9.80 & 21.62 & 17.97 & 0.56 & 0.56 \\
\hline S6026 & $\mathrm{O}=\mathrm{c} 1[\mathrm{nH}] \mathrm{ccn} 2 \mathrm{c} 1 \mathrm{nnn} 2$ & 2.91 & 2.65 & 13.29 & 12.22 & 23.48 & 22.38 & 0.68 & 0.62 \\
\hline S6027 & $\mathrm{O}=\mathrm{c} 1[\mathrm{nH}] \operatorname{ccc} 2 \mathrm{n} 1 \mathrm{nnn} 2$ & 2.87 & 2.65 & 13.83 & 11.63 & 23.96 & 21.19 & 0.57 & 0.63 \\
\hline S6029 & $\mathrm{O}=\mathrm{c} 1 \mathrm{ccn} 2 \mathrm{c}([\mathrm{nH}] 1) \mathrm{nnn} 2$ & 3.93 & 2.65 & 9.76 & 9.99 & 16.42 & 17.16 & 0.46 & 0.42 \\
\hline S603 & $\mathrm{O}=\mathrm{c} 1[\mathrm{nH}] \operatorname{ccc} 2 \mathrm{c} 1 \mathrm{occ} 2$ & -2.23 & -1.99 & 4.92 & 5.37 & 17.29 & 15.06 & 0.49 & 0.43 \\
\hline S6038 & $\mathrm{c} 1 \mathrm{ncn} 2 \mathrm{c}(\mathrm{n} 1) \mathrm{nnn} 2$ & 4.49 & 3.71 & 11.79 & 10.22 & 18.81 & 15.73 & 0.56 & 0.56 \\
\hline S6039 & $\mathrm{n} 1 \mathrm{cnn} 2 \mathrm{c}(\mathrm{c} 1) \mathrm{nnn} 2$ & 4.23 & 3.71 & 10.48 & 9.67 & 17.19 & 15.00 & 0.42 & 0.48 \\
\hline S605 & $\mathrm{O}=\mathrm{c} 1 \mathrm{ccc} 2 \mathrm{c}([\mathrm{nH}] 1) \operatorname{cco} 2$ & -1.24 & -1.99 & 4.96 & 5.09 & 16.73 & 14.48 & 0.41 & 0.45 \\
\hline S6069 & $\mathrm{O}=\mathrm{c} 1 \mathrm{cc}[\mathrm{nH}] \mathrm{c} 2 \mathrm{n} 1 \mathrm{nnn} 2$ & 2.81 & 2.65 & 14.65 & 12.79 & 24.40 & 24.06 & 0.57 & 0.48 \\
\hline
\end{tabular}




\begin{tabular}{|c|c|c|c|c|c|c|c|c|c|}
\hline S6073 & $\mathrm{c} 1 \mathrm{cnc} 2 \mathrm{n}(\mathrm{n} 1) \mathrm{nnn} 2$ & 4.11 & 3.71 & 13.40 & 10.25 & 22.17 & 16.43 & 0.73 & 0.70 \\
\hline S61 & $\mathrm{O}=\mathrm{c} 1 \mathrm{ncccs} 1$ & 0.67 & 0.49 & 7.71 & 6.65 & 18.32 & 17.28 & 0.42 & 0.49 \\
\hline S6104 & $\mathrm{O}=\mathrm{c} 1 \operatorname{cssc} 2 \mathrm{n} 1 \mathrm{nnn} 2$ & 3.56 & 3.56 & 12.04 & 10.29 & 20.29 & 18.38 & 0.58 & 0.55 \\
\hline S62 & $\mathrm{O}=\mathrm{c} 1 \mathrm{cncc}[\mathrm{nH}] 1$ & 0.14 & -0.42 & 8.26 & 6.36 & 16.71 & 15.42 & 0.48 & 0.42 \\
\hline S620 & $\mathrm{c} 1 \mathrm{ncc} 2 \mathrm{c}(\mathrm{n} 1) \mathrm{cco} 2$ & -0.96 & -0.93 & 4.72 & 3.88 & 10.29 & 10.70 & 0.39 & 0.45 \\
\hline S621 & $\mathrm{c} 1 \mathrm{ncc} 2 \mathrm{c}(\mathrm{n} 1) \operatorname{occ} 2$ & -0.79 & -0.93 & 4.45 & 4.33 & 10.45 & 11.15 & 0.64 & 0.61 \\
\hline S623 & c1cc2c(o1)nnnc2 & 1.50 & 0.90 & 8.19 & 8.06 & 18.51 & 16.35 & 0.77 & 0.76 \\
\hline S624 & $\mathrm{O}=\mathrm{c} 1[\mathrm{nH}] \mathrm{ncc} 2 \mathrm{c} 1 \mathrm{cco} 2$ & -0.42 & -0.16 & 4.51 & 4.76 & 13.59 & 11.40 & 0.50 & 0.46 \\
\hline S625 & $\mathrm{O}=\mathrm{c} 1[\mathrm{nH}] \mathrm{ncc} 2 \mathrm{c} 1 \mathrm{occ} 2$ & -0.29 & -0.16 & 5.56 & 5.00 & 15.83 & 11.93 & 0.41 & 0.47 \\
\hline S628 & $\mathrm{O}=\mathrm{c} 1 \mathrm{ncc} 2 \mathrm{c}([\mathrm{nH}] 1) \operatorname{cco} 2$ & 0.70 & -0.16 & 8.00 & 8.55 & 21.83 & 19.37 & 0.58 & 0.49 \\
\hline S653 & $\mathrm{O}=\mathrm{c} 1[\mathrm{nH}] \mathrm{c}(=\mathrm{O}) \mathrm{c} 2 \mathrm{c}([\mathrm{nH}] 1) \mathrm{cco} 2$ & 1.08 & 0.61 & 8.38 & 7.54 & 17.13 & 15.26 & 0.35 & 0.37 \\
\hline S66 & $\mathrm{O}=\mathrm{c} 1 \mathrm{cc}[\mathrm{nH}] \mathrm{c}(=\mathrm{O}) \mathrm{o} 1$ & 2.40 & 1.95 & 12.39 & 9.70 & 20.96 & 18.82 & 0.35 & 0.41 \\
\hline S664 & $\mathrm{O}=\mathrm{c} 1 \mathrm{cc}[\mathrm{nH}] \mathrm{c} 2 \mathrm{c} 1 \mathrm{cco} 2$ & -2.34 & -1.99 & 9.16 & 6.69 & 23.60 & 18.76 & 0.58 & 0.64 \\
\hline S666 & $\mathrm{O}=\mathrm{c} 1 \mathrm{nc}[\mathrm{nH}] \mathrm{c} 2 \mathrm{c} 1 \mathrm{cco} 2$ & -0.48 & -0.16 & 11.26 & 9.89 & 26.25 & 23.38 & 0.58 & 0.49 \\
\hline S667 & c1cnc2c(n1)occ2 & -1.26 & -0.93 & 3.12 & 3.80 & 8.21 & 10.11 & 0.49 & 0.43 \\
\hline S668 & c1nnc2c(n1)cco 2 & 0.98 & 0.90 & 6.98 & 5.92 & 14.41 & 11.77 & 0.38 & 0.41 \\
\hline S669 & $\mathrm{c} 1 \mathrm{nnc} 2 \mathrm{c}(\mathrm{n} 1) \operatorname{occ} 2$ & 0.93 & 0.90 & 6.47 & 5.65 & 13.48 & 11.51 & 0.54 & 0.48 \\
\hline S67 & $\mathrm{O}=\mathrm{c} 1[\mathrm{nH}] \mathrm{ccoc} 1=\mathrm{O}$ & 1.73 & 1.95 & 10.08 & 10.61 & 20.16 & 23.99 & 0.30 & 0.34 \\
\hline S670 & $\mathrm{O}=\mathrm{c} 1[\mathrm{nH}] \mathrm{cnc} 2 \mathrm{c} 1 \mathrm{occ} 2$ & -0.72 & -0.16 & 6.41 & 6.23 & 15.67 & 13.29 & 0.54 & 0.53 \\
\hline S671 & $\mathrm{O}=\mathrm{c} 1[\mathrm{nH}] \mathrm{cnc} 2 \mathrm{c} 1 \mathrm{cco} 2$ & -0.66 & -0.16 & 7.05 & 6.45 & 14.85 & 13.21 & 0.30 & 0.38 \\
\hline S673 & $\mathrm{O}=\mathrm{c} 1 \mathrm{cnc} 2 \mathrm{c}(\mathrm{o} 1) \mathrm{cco} 2$ & 1.46 & 1.44 & 5.96 & 6.33 & 13.23 & 12.80 & 0.35 & 0.45 \\
\hline S68 & $\mathrm{O}=\mathrm{c} 1 \mathrm{cc}[\mathrm{nH}] \mathrm{c}(=\mathrm{O}) \mathrm{s} 1$ & 1.43 & 1.26 & 11.10 & 8.66 & 18.23 & 17.33 & 0.71 & 0.67 \\
\hline S687 & $\mathrm{O}=\mathrm{c} 1[\mathrm{nH}] \mathrm{nnc} 2 \mathrm{c} 1 \mathrm{cco} 2$ & 1.26 & 1.67 & 5.46 & 7.36 & 11.12 & 12.58 & 0.39 & 0.44 \\
\hline S688 & $\mathrm{O}=\mathrm{c} 1[\mathrm{nH}] \mathrm{nnc} 2 \mathrm{c} 1 \mathrm{occ} 2$ & 1.17 & 1.67 & 5.77 & 6.88 & 12.68 & 12.41 & 0.76 & 0.72 \\
\hline S7 & c1ncc $[\mathrm{nH}] 1$ & -3.69 & -3.40 & 6.24 & 5.22 & 17.67 & 16.64 & 0.44 & 0.48 \\
\hline S704 & $\mathrm{O}=\mathrm{c} 1 \mathrm{cn}[\mathrm{nH}] \mathrm{c} 2 \mathrm{c} 1 \mathrm{cco} 2$ & -0.47 & -0.16 & 7.41 & 6.58 & 18.61 & 15.88 & 0.41 & 0.48 \\
\hline S71 & $\mathrm{O}=\mathrm{c} 1 \mathrm{cc}[\mathrm{nH}] \mathrm{cn} 1$ & -0.36 & -0.42 & 12.77 & 10.16 & 28.39 & 24.60 & 0.62 & 0.59 \\
\hline S712 & $\mathrm{O}=\mathrm{c} 1 \mathrm{nn}[\mathrm{nH}] \mathrm{c} 2 \mathrm{c} 1 \mathrm{cco} 2$ & 1.33 & 1.67 & 9.98 & 10.80 & 22.30 & 22.75 & 0.38 & 0.38 \\
\hline S72 & $\mathrm{O}=\mathrm{c} 1 \mathrm{nc}[\mathrm{nH}] \mathrm{cn} 1$ & 1.50 & 1.41 & 15.43 & 12.97 & 30.50 & 28.12 & 0.60 & 0.55 \\
\hline S73 & n1cence 1 & -0.91 & -1.19 & 1.62 & 2.95 & 5.38 & 10.27 & 0.71 & 0.79 \\
\hline S739 & $\mathrm{O}=\mathrm{c} 1 \mathrm{ccc} 2=\mathrm{c} 1 \mathrm{ccc} 2=\mathrm{O}$ & 1.10 & 1.11 & 2.93 & 5.82 & 6.84 & 13.85 & 0.45 & 0.51 \\
\hline
\end{tabular}




\begin{tabular}{|c|c|c|c|c|c|c|c|c|c|}
\hline S74 & n1ccnnc1 & 1.08 & 0.64 & 6.20 & 5.00 & 12.31 & 11.87 & 0.67 & 0.58 \\
\hline S75 & $\mathrm{O}=\mathrm{c} 1 \mathrm{ccnc}[\mathrm{nH}] 1$ & 0.12 & -0.42 & 7.34 & 5.40 & 15.54 & 13.34 & 0.64 & 0.71 \\
\hline S76 & $\mathrm{O}=\mathrm{c} 1 \mathrm{cncco} 1$ & 1.55 & 1.18 & 6.18 & 5.51 & 13.11 & 13.00 & 0.41 & 0.47 \\
\hline S77 & $\mathrm{O}=\mathrm{c} 1 \mathrm{ccnco} 1$ & 1.55 & 1.18 & 4.63 & 5.21 & 11.87 & 11.83 & 0.74 & 0.75 \\
\hline S79 & $\mathrm{O}=\mathrm{c} 1 \mathrm{ccncs} 1$ & 0.68 & 0.49 & 4.42 & 4.40 & 11.07 & 10.99 & 0.58 & 0.49 \\
\hline S7949 & $\operatorname{c} 1 \mathrm{ccc} 2 \mathrm{c}(\mathrm{c} 1) \mathrm{c}[\mathrm{nH}] \mathrm{c} 2$ & -6.74 & -6.19 & 4.45 & 2.46 & 17.68 & 14.22 & 0.87 & 0.93 \\
\hline S7950 & $\operatorname{c} 1 \mathrm{ccc} 2 \mathrm{c}(\mathrm{c} 1) \operatorname{coc} 2$ & -4.55 & -4.59 & -0.47 & 1.46 & 7.84 & 10.93 & 0.30 & 0.36 \\
\hline S7951 & $\operatorname{c} 1 \operatorname{ccc} 2 \mathrm{c}(\mathrm{c} 1) \csc 2$ & -4.95 & -5.28 & -0.61 & 0.71 & 8.15 & 10.30 & 0.37 & 0.36 \\
\hline S7952 & $\operatorname{c} 1 \mathrm{ccc} 2 \mathrm{c}(\mathrm{c} 1) \mathrm{n}[\mathrm{nH}] \mathrm{c} 2$ & -5.09 & -4.36 & 4.22 & 3.37 & 14.31 & 13.78 & 0.49 & 0.66 \\
\hline S7953 & c1ccc $2 \mathrm{c}(\mathrm{c} 1) \operatorname{noc} 2$ & -2.55 & -2.76 & 3.17 & 3.33 & 12.13 & 11.82 & 0.33 & 0.37 \\
\hline S7954 & $\operatorname{c} 1 \operatorname{ccc} 2 \mathrm{c}(\mathrm{c} 1) \mathrm{nsc} 2$ & -3.39 & -3.45 & 2.68 & 2.52 & 10.57 & 11.03 & 0.40 & 0.45 \\
\hline S7955 & $\operatorname{c} 1 \operatorname{ccc} 2 \mathrm{c}(\mathrm{c} 1) \mathrm{n}[\mathrm{nH}] \mathrm{n} 2$ & -3.39 & -2.53 & 3.02 & 3.26 & 9.11 & 11.08 & 0.53 & 0.47 \\
\hline S7956 & c1ccc2c(c1)non2 & -0.50 & -0.93 & 4.76 & 4.17 & 11.18 & 10.47 & 0.42 & 0.47 \\
\hline S7957 & $\operatorname{c} 1 \mathrm{ccc} 2 \mathrm{c}(\mathrm{c} 1) \mathrm{nsn} 2$ & -1.78 & -1.62 & 2.00 & 3.30 & 6.05 & 9.51 & 0.63 & 0.64 \\
\hline S7958 & $\operatorname{c} 1 \operatorname{ccc} 2 \mathrm{c}(\mathrm{c} 1) \operatorname{ccc} 2$ & -5.16 & -5.81 & -1.20 & 0.73 & 7.73 & 11.22 & 0.56 & 0.50 \\
\hline S7959 & $\operatorname{c} 1 \mathrm{ccc} 2 \mathrm{c}(\mathrm{c} 1) \operatorname{cnc} 2$ & -3.17 & -3.98 & 1.66 & 2.35 & 9.92 & 11.87 & 0.51 & 0.47 \\
\hline S7960 & $\operatorname{c1ccc} 2 \mathrm{c}(\mathrm{c} 1) \mathrm{nccc} 2$ & -3.91 & -3.98 & 1.66 & 2.35 & 10.24 & 11.87 & 0.58 & 0.51 \\
\hline S7961 & c1ccc $2 \mathrm{c}(\mathrm{c} 1) \mathrm{nncc} 2$ & -1.85 & -2.15 & 4.01 & 4.99 & 14.36 & 14.77 & 0.65 & 0.56 \\
\hline S7962 & $\operatorname{c} 1 \mathrm{ccc} 2 \mathrm{c}(\mathrm{c} 1) \mathrm{cnn} c 2$ & -0.90 & -2.15 & 4.71 & 4.99 & 16.17 & 14.77 & 0.53 & 0.47 \\
\hline S7963 & $\mathrm{O}=\mathrm{c} 1[\mathrm{nH}] \mathrm{ccc} 2 \mathrm{c} 1 \mathrm{ccc} 2$ & -3.38 & -3.21 & 4.47 & 4.24 & 15.22 & 14.82 & 0.53 & 0.51 \\
\hline S7964 & $\mathrm{O}=\mathrm{c} 1 \mathrm{ccc} 2 \mathrm{c}([\mathrm{nH}] 1) \operatorname{ccc} 2$ & -1.76 & -3.21 & 3.01 & 4.24 & 14.02 & 14.82 & 0.64 & 0.62 \\
\hline S7966 & $\mathrm{O}=\mathrm{c} 1 \mathrm{occc} 2 \mathrm{c} 1 \mathrm{ccc} 2$ & -1.65 & -1.61 & 3.73 & 4.94 & 13.95 & 14.56 & 0.39 & 0.46 \\
\hline S7967 & $\mathrm{O}=\mathrm{c} 1 \mathrm{ccc} 2 \mathrm{c}(\mathrm{o} 1) \operatorname{ccc} 2$ & -0.93 & -1.61 & 4.83 & 4.94 & 15.46 & 14.56 & 0.63 & 0.56 \\
\hline S7970 & $\mathrm{O}=\mathrm{c} 1 \operatorname{ccc} 2 \mathrm{c}(\mathrm{s} 1) \operatorname{ccc} 2$ & -1.45 & -2.30 & 4.33 & 4.08 & 14.40 & 13.56 & 0.48 & 0.41 \\
\hline S7972 & c1ccc2c(c1)ncnc2 & -1.84 & -2.15 & 3.00 & 3.38 & 9.09 & 11.22 & 0.67 & 0.59 \\
\hline S7973 & c1ccc $2 c(c 1) n n n c 2$ & 0.40 & -0.32 & 6.41 & 7.04 & 17.20 & 16.37 & 0.63 & 0.60 \\
\hline S7974 & $\mathrm{O}=\mathrm{c} 1[\mathrm{nH}] \mathrm{ncc} 2 \mathrm{c} 1 \mathrm{ccc} 2$ & -1.36 & -1.38 & 4.00 & 4.07 & 13.31 & 11.88 & 0.68 & 0.58 \\
\hline S7982 & $\mathrm{O}=\mathrm{c} 1 \mathrm{snc} 2 \mathrm{c} 1 \mathrm{ccc} 2$ & -0.33 & -0.47 & 5.59 & 5.35 & 13.81 & 12.52 & 0.75 & 0.69 \\
\hline S7984 & $\mathrm{O}=\mathrm{c} 1 \mathrm{n} \operatorname{cc} 2 \mathrm{c}(\mathrm{s} 1) \operatorname{ccc} 2$ & 0.31 & -0.47 & 6.14 & 6.65 & 17.65 & 17.28 & 0.49 & 0.52 \\
\hline S7986 & $\mathrm{O}=\mathrm{c} 1 \mathrm{cnc} 2 \mathrm{c}([\mathrm{nH}] 1) \mathrm{cccc} 2$ & -0.59 & -1.38 & 5.25 & 6.36 & 13.44 & 15.42 & 0.59 & 0.51 \\
\hline S7987 & $\mathrm{O}=\mathrm{c} 1 \mathrm{nnn} 2 \mathrm{c}([\mathrm{nH}] 1) \operatorname{cccc} 2$ & 1.31 & 0.45 & 8.23 & 10.79 & 19.18 & 22.49 & 0.54 & 0.47 \\
\hline
\end{tabular}




\begin{tabular}{|c|c|c|c|c|c|c|c|c|c|}
\hline S7988 & $\mathrm{O}=\mathrm{c} 1[\mathrm{nH}] \mathrm{c} 2 \operatorname{ccccc} 2 \mathrm{c}(=\mathrm{O})[\mathrm{nH}] 1$ & 0.14 & -0.61 & 6.39 & 6.31 & 13.53 & 15.07 & 0.59 & 0.53 \\
\hline S7990 & $\mathrm{O}=\mathrm{c} 1 \mathrm{oc}(=\mathrm{O}) \mathrm{c} 2 \mathrm{c}([\mathrm{nH}] 1) \operatorname{cccc} 2$ & 1.67 & 0.99 & 8.66 & 9.70 & 17.08 & 18.82 & 0.62 & 0.54 \\
\hline S7992 & $\mathrm{O}=\mathrm{c} 1 \mathrm{sc}(=\mathrm{O}) \mathrm{c} 2 \mathrm{c}([\mathrm{nH}] 1) \operatorname{ccc} 2$ & 0.72 & 0.30 & 7.53 & 8.66 & 14.42 & 17.33 & 0.46 & 0.59 \\
\hline S7994 & $\mathrm{O}=\mathrm{c} 1 \mathrm{cc}[\mathrm{nH}] \mathrm{c} 2 \mathrm{c} 1 \mathrm{ccc} 2$ & -3.16 & -3.21 & 8.61 & 6.76 & 22.75 & 19.78 & 0.53 & 0.44 \\
\hline S7995 & $\mathrm{O}=\mathrm{c} 1 \mathrm{nc}[\mathrm{nH}] \mathrm{c} 2 \mathrm{c} 1 \mathrm{ccc} 2$ & -1.26 & -1.38 & 10.68 & 10.16 & 25.64 & 24.60 & 0.34 & 0.41 \\
\hline S7996 & $\operatorname{c1ccc} 2 \mathrm{c}(\mathrm{c} 1) \mathrm{nccn} 2$ & -2.66 & -2.15 & 1.39 & 2.95 & 6.32 & 10.27 & 0.50 & 0.43 \\
\hline S7997 & c1cce $2 \mathrm{c}(\mathrm{c} 1) \mathrm{nncn} 2$ & -0.52 & -0.32 & 4.31 & 5.00 & 11.45 & 11.87 & 0.70 & 0.60 \\
\hline S7998 & $\mathrm{O}=\mathrm{c} 1[\mathrm{nH}] \mathrm{cnc} 2 \mathrm{c} 1 \mathrm{cccc} 2$ & -2.12 & -1.38 & 5.82 & 5.40 & 13.67 & 13.34 & 0.33 & 0.38 \\
\hline S7999 & $\mathrm{O}=\mathrm{c} 1 \mathrm{cnc} 2 \mathrm{c}(\mathrm{o} 1) \operatorname{ccc} 2$ & 0.23 & 0.22 & 3.70 & 5.51 & 11.11 & 13.00 & 0.64 & 0.71 \\
\hline S80 & n1cncnc1 & 1.06 & 0.64 & 2.52 & 3.82 & 3.82 & 9.28 & 0.70 & 0.60 \\
\hline S8000 & $\mathrm{O}=\mathrm{c} 1 \mathrm{ocn} \operatorname{2c} 1 \mathrm{ccc} 2$ & -0.39 & 0.22 & 3.35 & 5.21 & 10.88 & 11.83 & 0.36 & 0.35 \\
\hline S8002 & $\mathrm{O}=\mathrm{c} 1 \mathrm{scnc} 2 \mathrm{c} 1 \mathrm{ccc} 2$ & -1.25 & -0.47 & 2.69 & 4.40 & 9.59 & 10.99 & 0.56 & 0.48 \\
\hline S8003 & c1ccc2c(c1)nnnn2 & 1.75 & 1.51 & 7.67 & 8.07 & 16.07 & 15.72 & 0.44 & 0.55 \\
\hline S8004 & $\mathrm{O}=\mathrm{c} 1[\mathrm{nH}][\mathrm{n}] \mathrm{c} 2 \mathrm{c}([\mathrm{n}] 1) \mathrm{cccc} 2$ & 0.44 & 0.45 & 5.52 & 6.45 & 17.60 & 14.46 & 0.62 & 0.57 \\
\hline S8007 & $\mathrm{O}=\mathrm{c} 1[\mathrm{nH}] \mathrm{nnc} 2 \mathrm{c} 1 \mathrm{cccc} 2$ & -0.17 & 0.45 & 4.09 & 6.25 & 10.34 & 12.65 & 0.67 & 0.60 \\
\hline S8010 & $\mathrm{O}=\mathrm{c} 1 \mathrm{sn}[\mathrm{n}] \mathrm{c} 2 \mathrm{c} 1 \mathrm{ccc} 2$ & 0.66 & 1.36 & 5.76 & 6.70 & 11.40 & 12.19 & 0.54 & 0.50 \\
\hline S8012 & $\mathrm{O}=\mathrm{c} 1 \mathrm{ccoc} 2 \mathrm{c} 1 \mathrm{ccc} 2$ & -1.99 & -1.61 & 3.89 & 4.06 & 14.31 & 13.46 & 0.35 & 0.42 \\
\hline S8013 & $\mathrm{O}=\mathrm{c} 1 \mathrm{n} \operatorname{coc} 2 \mathrm{c} 1 \mathrm{cccc} 2$ & -0.12 & 0.22 & 4.88 & 6.57 & 16.15 & 17.03 & 0.62 & 0.54 \\
\hline S8014 & $\mathrm{O}=\mathrm{c} 1 \mathrm{c} \csc 2 \mathrm{c} 1 \mathrm{ccc} 2$ & -2.44 & -2.30 & 3.04 & 3.42 & 13.14 & 13.20 & 0.43 & 0.40 \\
\hline S8015 & $\mathrm{O}=\mathrm{c} 1 \mathrm{cn}[\mathrm{nH}] \mathrm{c} 2 \mathrm{c} 1 \mathrm{ccc} 2$ & -1.34 & -1.38 & 6.96 & 6.58 & 17.83 & 16.84 & 0.47 & 0.40 \\
\hline S8017 & $\mathrm{O}=\mathrm{c} 1 \mathrm{cnsc} 2 \mathrm{c} 1 \mathrm{ccc} 2$ & -0.59 & -0.47 & 4.49 & 4.69 & 11.81 & 12.16 & 0.50 & 0.46 \\
\hline S8018 & $\mathrm{O}=\mathrm{c} 1 \mathrm{n} \csc 2 \mathrm{c} 1 \mathrm{ccc} 2$ & -0.75 & -0.47 & 4.78 & 5.99 & 16.17 & 16.93 & 0.32 & 0.43 \\
\hline S8019 & $\mathrm{O}=\mathrm{c} 1 \mathrm{nn}[\mathrm{nH}] \mathrm{c} 2 \mathrm{c} 1 \mathrm{cccc} 2$ & 0.54 & 0.45 & 9.57 & 11.01 & 22.14 & 23.91 & 0.64 & 0.70 \\
\hline S8021 & $\mathrm{O}=\mathrm{c} 1 \mathrm{nnnsc} 2 \mathrm{c} 1 \mathrm{ccc} 2$ & 1.06 & 1.36 & 6.75 & 8.28 & 15.81 & 18.13 & 0.50 & 0.44 \\
\hline $\mathbf{S 8 0 2 3}$ & $\mathrm{O}=\mathrm{c} 1 \mathrm{oc} 2 \operatorname{cccc} 2 \mathrm{c}(=\mathrm{O})[\mathrm{nH}] 1$ & 1.12 & 0.99 & 5.00 & 6.31 & 11.56 & 12.76 & 0.58 & 0.55 \\
\hline S8024 & $\mathrm{O}=\mathrm{c} 1 \mathrm{sc} 2 \operatorname{cccc} 2 \mathrm{c}(=\mathrm{O})[\mathrm{nH}] 1$ & 0.42 & 0.30 & 5.13 & 5.49 & 11.62 & 12.01 & 0.37 & 0.37 \\
\hline S8030 & $\mathrm{O}=\mathrm{c} 1 \mathrm{oc} 2 \operatorname{cccc} 2 \mathrm{c}(=\mathrm{O}) \mathrm{s} 1$ & 1.51 & 1.90 & 6.11 & 7.40 & 12.44 & 13.97 & 0.78 & 0.82 \\
\hline S8032 & $\mathrm{O}=\mathrm{c} 1 \mathrm{oc}(=\mathrm{O}) \mathrm{c} 2 \mathrm{c}(\mathrm{s} 1) \operatorname{ccc} 2$ & 1.79 & 1.90 & 7.17 & 7.62 & 14.75 & 14.71 & 0.39 & 0.50 \\
\hline S8035 & $\mathrm{c} 1 \mathrm{ncc} 2 \mathrm{c}(\mathrm{c} 1) \mathrm{c}[\mathrm{nH}] \mathrm{c} 2$ & -4.73 & -4.36 & 7.28 & 4.68 & 19.58 & 15.80 & 0.46 & 0.45 \\
\hline S8036 & $\mathrm{c} 1 \mathrm{cnc} 2 \mathrm{c}(\mathrm{c} 1) \mathrm{c}[\mathrm{nH}] \mathrm{c} 2$ & -5.24 & -4.36 & 5.40 & 4.08 & 17.37 & 14.87 & 0.39 & 0.48 \\
\hline S8037 & $\mathrm{c} 1 \mathrm{ncc} 2 \mathrm{c}(\mathrm{c} 1) \operatorname{coc} 2$ & -2.59 & -2.76 & 2.32 & 2.87 & 10.22 & 11.37 & 0.44 & 0.50 \\
\hline
\end{tabular}




\begin{tabular}{|c|c|c|c|c|c|c|c|c|c|}
\hline S8038 & $\mathrm{c} 1 \mathrm{cnc} 2 \mathrm{c}(\mathrm{c} 1) \operatorname{coc} 2$ & -3.03 & -2.76 & 2.34 & 3.08 & 10.42 & 11.58 & 0.59 & 0.57 \\
\hline S8039 & $\operatorname{c1ncc} 2 \mathrm{c}(\mathrm{c} 1) \csc 2$ & -2.99 & -3.45 & 1.97 & 2.17 & 10.36 & 10.88 & 0.77 & 0.82 \\
\hline S8040 & $\mathrm{c} 1 \mathrm{cnc} 2 \mathrm{c}(\mathrm{c} 1) \csc 2$ & -3.57 & -3.45 & 1.88 & 2.33 & 10.44 & 10.95 & 0.56 & 0.61 \\
\hline S8041 & $\operatorname{c1ncc} 2 \mathrm{c}(\mathrm{c} 1) \mathrm{n}[\mathrm{nH}] \mathrm{c} 2$ & -3.09 & -2.53 & 7.24 & 5.08 & 16.33 & 14.23 & 0.45 & 0.41 \\
\hline S8042 & $\operatorname{c} 1 \mathrm{ncc} 2 \mathrm{c}(\mathrm{c} 1) \mathrm{c}[\mathrm{nH}] \mathrm{n} 2$ & -3.07 & -2.53 & 7.16 & 5.75 & 17.03 & 15.70 & 0.44 & 0.50 \\
\hline S8043 & $\mathrm{c} 1 \mathrm{cnc} 2 \mathrm{c}(\mathrm{c} 1) \mathrm{c}[\mathrm{nH}] \mathrm{n} 2$ & -3.33 & -2.53 & 5.73 & 6.07 & 18.49 & 16.79 & 0.58 & 0.53 \\
\hline S8044 & $\mathrm{c} 1 \mathrm{cnc} 2 \mathrm{c}(\mathrm{c} 1) \mathrm{n}[\mathrm{nH}] \mathrm{c} 2$ & -3.58 & -2.53 & 5.00 & 4.24 & 12.99 & 12.76 & 0.54 & 0.55 \\
\hline S8045 & c1ncc2c(c1)noc2 & -0.62 & -0.93 & 6.04 & 4.23 & 12.03 & 11.15 & 0.36 & 0.43 \\
\hline S8046 & $\mathrm{c} 1 \mathrm{ncc} 2 \mathrm{c}(\mathrm{c} 1) \operatorname{con} 2$ & -0.61 & -0.93 & 5.78 & 4.90 & 11.49 & 12.61 & 0.59 & 0.54 \\
\hline S8047 & $\mathrm{c} 1 \mathrm{cnc} 2 \mathrm{c}(\mathrm{c} 1) \operatorname{con} 2$ & -0.76 & -0.93 & 6.33 & 6.02 & 17.32 & 14.84 & 0.67 & 0.63 \\
\hline S8049 & $\mathrm{c} 1 \mathrm{ncc} 2 \mathrm{c}(\mathrm{c} 1) \mathrm{nsc} 2$ & -1.43 & -1.62 & 5.30 & 3.47 & 11.22 & 10.49 & 0.47 & 0.51 \\
\hline S8050 & $\operatorname{c} 1 n \operatorname{cc} 2 \mathrm{c}(\mathrm{c} 1) \operatorname{csn} 2$ & -1.41 & -1.62 & 5.36 & 4.14 & 12.30 & 11.96 & 0.32 & 0.40 \\
\hline S8051 & $\operatorname{c} 1 \mathrm{cnc} 2 \mathrm{c}(\mathrm{c} 1) \operatorname{csn} 2$ & -1.72 & -1.62 & 5.11 & 5.21 & 15.96 & 14.04 & 0.51 & 0.42 \\
\hline S8053 & $\mathrm{c} 1 \mathrm{ncc} 2 \mathrm{c}(\mathrm{c} 1) \mathrm{n}[\mathrm{nH}] \mathrm{n} 2$ & -1.42 & -0.70 & 5.92 & 5.13 & 12.34 & 11.89 & 0.52 & 0.45 \\
\hline S8054 & $\mathrm{c} 1 \mathrm{cnc} 2 \mathrm{c}(\mathrm{c} 1) \mathrm{n}[\mathrm{nH}] \mathrm{n} 2$ & -1.64 & -0.70 & 4.05 & 5.20 & 12.67 & 12.43 & 0.46 & 0.47 \\
\hline S8055 & c1ncc2c(c1)non2 & 1.37 & 0.90 & 4.11 & 5.23 & 7.28 & 10.14 & 0.57 & 0.52 \\
\hline S8056 & c1cnc2c(c1)non2 & 1.26 & 0.90 & 7.66 & 6.11 & 14.65 & 11.82 & 0.55 & 0.51 \\
\hline S8057 & $\mathrm{c} 1 \mathrm{ncc} 2 \mathrm{c}(\mathrm{c} 1) \mathrm{nsn} 2$ & 0.17 & 0.21 & 2.82 & 4.41 & 7.09 & 9.32 & 0.34 & 0.41 \\
\hline S8058 & $\mathrm{c} 1 \mathrm{cnc} 2 \mathrm{c}(\mathrm{c} 1) \mathrm{nsn} 2$ & -0.10 & 0.21 & 4.63 & 5.24 & 11.68 & 10.86 & 0.79 & 0.73 \\
\hline S8059 & $\mathrm{n} 1 \mathrm{ccc} 2 \mathrm{c}(\mathrm{c} 1) \mathrm{cncc} 2$ & -1.20 & -2.15 & 3.16 & 3.68 & 8.25 & 11.87 & 0.32 & 0.36 \\
\hline S8060 & c1ncc2c(c1)cncc2 & -1.21 & -2.15 & 2.40 & 3.41 & 7.29 & 11.28 & 0.45 & 0.55 \\
\hline S8061 & $\mathrm{c} 1 \mathrm{cnc} 2 \mathrm{c}(\mathrm{c} 1) \mathrm{cncc} 2$ & -1.89 & -2.15 & 3.80 & 3.63 & 10.33 & 11.78 & 0.32 & 0.40 \\
\hline S8062 & $\mathrm{c} 1 \mathrm{cnc} 2 \mathrm{c}(\mathrm{c} 1) \mathrm{ccnc} 2$ & -1.86 & -2.15 & 4.44 & 4.35 & 12.26 & 13.36 & 0.73 & 0.62 \\
\hline S8063 & $\mathrm{c} 1 \mathrm{cnc} 2 \mathrm{c}(\mathrm{c} 1) \operatorname{ccc} 2$ & -2.35 & -2.15 & 4.23 & 5.12 & 16.06 & 15.04 & 0.40 & 0.53 \\
\hline S8064 & $\mathrm{c} 1 \mathrm{cnc} 2 \mathrm{c}(\mathrm{c} 1) \mathrm{nccc} 2$ & -2.67 & -2.15 & 1.54 & 3.21 & 7.25 & 10.85 & 0.45 & 0.54 \\
\hline S8065 & $\mathrm{n} 1 \mathrm{ccc} 2 \mathrm{c}(\mathrm{c} 1) \mathrm{nncc} 2$ & 0.15 & -0.32 & 6.74 & 6.70 & 14.20 & 15.61 & 0.49 & 0.44 \\
\hline S8066 & $\mathrm{n} 1 \mathrm{ccc} 2 \mathrm{c}(\mathrm{c} 1) \mathrm{ccnn} 2$ & 0.12 & -0.32 & 6.11 & 5.71 & 13.22 & 13.44 & 0.69 & 0.62 \\
\hline S8067 & c1cnc2c(c1)nncc2 & -0.61 & -0.32 & 4.58 & 5.51 & 12.85 & 13.00 & 0.66 & 0.58 \\
\hline S8068 & $\mathrm{c} 1 \mathrm{cnc} 2 \mathrm{c}(\mathrm{c} 1) \mathrm{ccnn} 2$ & -0.23 & -0.32 & 7.39 & 8.14 & 19.48 & 18.78 & 0.80 & 0.83 \\
\hline S8069 & $\mathrm{n} 1 \mathrm{ccc} 2 \mathrm{c}(\mathrm{c} 1) \mathrm{cnnc} 2$ & 1.02 & -0.32 & 5.59 & 5.75 & 13.86 & 13.53 & 0.29 & 0.33 \\
\hline $\mathbf{S 8 0 7 0}$ & $\mathrm{c} 1 \mathrm{ccc} 2 \mathrm{c}(\mathrm{n} 1) \mathrm{cnnc} 2$ & 0.42 & -0.32 & 7.24 & 6.66 & 17.62 & 15.52 & 0.45 & 0.50 \\
\hline
\end{tabular}




\begin{tabular}{|c|c|c|c|c|c|c|c|c|c|}
\hline S8071 & $\mathrm{O}=\mathrm{c} 1[\mathrm{nH}] \mathrm{ccc} 2 \mathrm{c} 1 \mathrm{cnc} 2$ & -1.34 & -1.38 & 7.38 & 6.61 & 16.51 & 17.16 & 0.66 & 0.57 \\
\hline S8072 & $\mathrm{O}=\mathrm{c} 1[\mathrm{nH}] \mathrm{ccc} 2 \mathrm{c} 1 \mathrm{ccnc} 2$ & -1.30 & -1.38 & 6.94 & 6.13 & 14.76 & 15.41 & 0.58 & 0.53 \\
\hline S8073 & $\mathrm{O}=\mathrm{c} 1[\mathrm{nH}] \operatorname{ccc} 2 \mathrm{c} 1 \mathrm{ccc} 2$ & -2.02 & -1.38 & 5.31 & 5.41 & 13.53 & 13.71 & 0.64 & 0.52 \\
\hline S8074 & $\mathrm{O}=\mathrm{c} 1[\mathrm{nH}] \operatorname{ccc} 2 \mathrm{c} 1 \mathrm{nccc} 2$ & -2.09 & -1.38 & 5.61 & 6.70 & 19.71 & 18.35 & 0.32 & 0.44 \\
\hline S8075 & $\mathrm{O}=\mathrm{c} 1 \mathrm{ccc} 2 \mathrm{c}([\mathrm{nH}] 1) \mathrm{ccnc} 2$ & 0.18 & -1.38 & 5.31 & 5.62 & 13.38 & 14.32 & 0.38 & 0.45 \\
\hline S8076 & $\mathrm{O}=\mathrm{c} 1 \mathrm{ccc} 2 \mathrm{c}([\mathrm{nH}] 1) \mathrm{cncc} 2$ & 0.20 & -1.38 & 5.26 & 5.11 & 13.67 & 13.90 & 0.71 & 0.69 \\
\hline S8077 & $\mathrm{O}=\mathrm{c} 1 \mathrm{ccc} 2 \mathrm{c}([\mathrm{nH}] 1) \mathrm{nccc} 2$ & -0.57 & -1.38 & 5.73 & 5.00 & 16.25 & 14.66 & 0.59 & 0.56 \\
\hline S8078 & $\mathrm{O}=\mathrm{c} 1 \mathrm{ccc} 2 \mathrm{c}([\mathrm{nH}] 1) \operatorname{ccc} 2$ & -0.52 & -1.38 & 6.12 & 6.35 & 15.50 & 15.75 & 0.63 & 0.62 \\
\hline $\mathbf{S 8 0 8 3}$ & $\mathrm{O}=\mathrm{c} 1 \mathrm{occc} 2 \mathrm{c} 1 \mathrm{cncc} 2$ & 0.36 & 0.22 & 6.53 & 6.86 & 14.93 & 16.27 & 0.61 & 0.57 \\
\hline S8084 & $\mathrm{O}=\mathrm{c} 1 \mathrm{occc} 2 \mathrm{c} 1 \mathrm{ccnc} 2$ & 0.39 & 0.22 & 5.37 & 5.97 & 12.67 & 13.96 & 0.31 & 0.37 \\
\hline S8085 & $\mathrm{O}=\mathrm{c} 1 \mathrm{occc} 2 \mathrm{c} 1 \mathrm{ccc} 2$ & -0.29 & 0.22 & 3.71 & 5.59 & 11.67 & 12.74 & 0.51 & 0.56 \\
\hline S8086 & $\mathrm{O}=\mathrm{c} 1 \mathrm{occc} 2 \mathrm{c} 1 \mathrm{nccc} 2$ & -0.35 & 0.22 & 6.71 & 7.97 & 19.17 & 18.89 & 0.29 & 0.33 \\
\hline S8087 & $\mathrm{O}=\mathrm{c} 1 \mathrm{ccc} 2 \mathrm{c}(\mathrm{o} 1) \operatorname{ccnc} 2$ & 1.03 & 0.22 & 6.32 & 5.81 & 14.12 & 13.34 & 0.60 & 0.59 \\
\hline S8088 & $\mathrm{O}=\mathrm{c} 1 \mathrm{ccc} 2 \mathrm{c}(\mathrm{o} 1) \mathrm{cncc} 2$ & 1.09 & 0.22 & 7.77 & 6.39 & 15.94 & 14.45 & 0.53 & 0.59 \\
\hline S8090 & $\mathrm{O}=\mathrm{c} 1 \operatorname{ccc} 2 \mathrm{c}(\mathrm{o} 1) \operatorname{ccc} 2$ & 0.30 & 0.22 & 4.84 & 5.89 & 13.64 & 13.88 & 0.49 & 0.55 \\
\hline S81 & c1ncnnn 1 & 2.97 & 2.47 & 6.44 & 6.46 & 11.18 & 12.17 & 0.65 & 0.58 \\
\hline S8107 & $\mathrm{n} 1 \mathrm{cnc} 2 \mathrm{c}(\mathrm{c} 1) \mathrm{ccnc} 2$ & 0.16 & -0.32 & 5.20 & 4.82 & 9.60 & 11.47 & 0.45 & 0.54 \\
\hline S8108 & c1ncc $2 \mathrm{c}(\mathrm{c} 1) \mathrm{ncnc} 2$ & 0.13 & -0.32 & 4.08 & 4.37 & 7.34 & 10.49 & 0.56 & 0.51 \\
\hline S8109 & $\mathrm{c} 1 \mathrm{cnc} 2 \mathrm{c}(\mathrm{c} 1) \mathrm{ncnc} 2$ & -0.56 & -0.32 & 4.66 & 4.62 & 9.52 & 11.04 & 0.48 & 0.55 \\
\hline S8110 & $\mathrm{c} 1 \mathrm{cnc} 2 \mathrm{c}(\mathrm{c} 1) \mathrm{cncn} 2$ & -0.27 & -0.32 & 6.36 & 5.81 & 15.37 & 13.66 & 0.71 & 0.64 \\
\hline S8111 & c1ncc2c(c1)cnnn2 & 2.35 & 1.51 & 8.51 & 8.19 & 16.28 & 15.97 & 0.53 & 0.47 \\
\hline S8112 & $\mathrm{n} 1 \mathrm{ccc} 2 \mathrm{c}(\mathrm{c} 1) \mathrm{cnnn} 2$ & 2.31 & 1.51 & 7.33 & 7.47 & 14.85 & 14.39 & 0.80 & 0.66 \\
\hline S8113 & c1cnc2c(c1)nnnc2 & 1.68 & 1.51 & 8.01 & 7.95 & 17.11 & 15.44 & 0.42 & 0.48 \\
\hline S8114 & $\mathrm{c} 1 \mathrm{cnc} 2 \mathrm{c}(\mathrm{c} 1) \mathrm{cnnn} 2$ & 2.02 & 1.51 & 9.81 & 9.86 & 20.37 & 19.64 & 0.33 & 0.40 \\
\hline S8115 & $\mathrm{O}=\mathrm{c} 1[\mathrm{nH}] \mathrm{ncc} 2 \mathrm{c} 1 \mathrm{cncc} 2$ & 0.61 & 0.45 & 6.22 & 5.88 & 13.75 & 12.98 & 0.30 & 0.34 \\
\hline S8116 & $\mathrm{O}=\mathrm{c} 1[\mathrm{nH}] \mathrm{ncc} 2 \mathrm{c} 1 \mathrm{ccnc} 2$ & 0.66 & 0.45 & 4.62 & 5.66 & 11.05 & 11.83 & 0.32 & 0.42 \\
\hline S8117 & $\mathrm{O}=\mathrm{c} 1[\mathrm{nH}] \mathrm{ncc} 2 \mathrm{c} 1 \mathrm{ccc} 2$ & 0.01 & 0.45 & 4.65 & 5.61 & 11.52 & 11.61 & 0.38 & 0.38 \\
\hline S8118 & $\mathrm{O}=\mathrm{c} 1[\mathrm{nH}] \mathrm{ncc} 2 \mathrm{c} 1 \mathrm{nccc} 2$ & -0.07 & 0.45 & 7.12 & 6.19 & 19.22 & 14.67 & 0.81 & 0.83 \\
\hline $\mathbf{S 8 1 2 3}$ & $\mathrm{O}=\mathrm{c} 1 \mathrm{ncc} 2 \mathrm{c}([\mathrm{nH}] 1) \mathrm{cncc} 2$ & 2.08 & 0.45 & 6.91 & 7.95 & 17.22 & 17.48 & 0.53 & 0.50 \\
\hline S8124 & $\mathrm{O}=\mathrm{c} 1 \mathrm{ncc} 2 \mathrm{c}([\mathrm{nH}] 1) \mathrm{ccnc} 2$ & 2.08 & 0.45 & 7.99 & 8.73 & 18.17 & 18.49 & 0.44 & 0.51 \\
\hline $\mathbf{S 8 1 2 5}$ & $\mathrm{O}=\mathrm{c} 1 \mathrm{ncc} 2 \mathrm{c}([\mathrm{nH}] 1) \operatorname{ccc} 2$ & 1.45 & 0.45 & 8.88 & 10.13 & 20.58 & 21.41 & 0.60 & 0.53 \\
\hline
\end{tabular}




\begin{tabular}{|c|c|c|c|c|c|c|c|c|c|}
\hline S8126 & $\mathrm{O}=\mathrm{c} 1 \mathrm{ncc} 2 \mathrm{c}([\mathrm{nH}] 1) \mathrm{nccc} 2$ & 1.35 & 0.45 & 7.81 & 8.06 & 20.00 & 18.74 & 0.48 & 0.52 \\
\hline S8149 & $\mathrm{O}=\mathrm{c} 1 \mathrm{sncc} 2 \mathrm{c} 1 \mathrm{ccc} 2$ & 0.93 & 1.36 & 6.37 & 6.42 & 12.39 & 11.62 & 0.41 & 0.45 \\
\hline $\mathbf{S 8 1 5 0}$ & $\mathrm{O}=\mathrm{c} 1 \mathrm{sncc} 2 \mathrm{c} 1 \mathrm{nccc} 2$ & 0.88 & 1.36 & 8.63 & 8.01 & 19.01 & 16.01 & 0.62 & 0.66 \\
\hline S8163 & $\mathrm{O}=\mathrm{c} 1 \mathrm{cnc} 2 \mathrm{c}([\mathrm{nH}] 1) \mathrm{ccnc} 2$ & 1.43 & 0.45 & 7.96 & 8.12 & 13.25 & 15.76 & 0.41 & 0.47 \\
\hline S8164 & $\mathrm{O}=\mathrm{c} 1 \mathrm{cnc} 2 \mathrm{c}([\mathrm{nH}] 1) \mathrm{cncc} 2$ & 1.40 & 0.45 & 6.45 & 6.89 & 12.02 & 13.77 & 0.56 & 0.63 \\
\hline S8165 & $\mathrm{O}=\mathrm{c} 1 \mathrm{cnc} 2 \mathrm{c}([\mathrm{nH}] 1) \mathrm{nccc} 2$ & 0.60 & 0.45 & 4.48 & 6.35 & 12.10 & 13.59 & 0.77 & 0.74 \\
\hline S8166 & $\mathrm{O}=\mathrm{c} 1 \mathrm{cnc} 2 \mathrm{c}([\mathrm{nH}] 1) \operatorname{ccc} 2$ & 0.97 & 0.45 & 8.54 & 9.61 & 15.63 & 18.88 & 0.53 & 0.44 \\
\hline S8167 & $\mathrm{O}=\mathrm{c} 1 \mathrm{nnc} 2 \mathrm{c}([\mathrm{nH}] 1) \mathrm{ccnc} 2$ & 3.28 & 2.28 & 11.03 & 12.25 & 18.98 & 22.18 & 0.61 & 0.55 \\
\hline S8168 & $\mathrm{O}=\mathrm{c} 1 \mathrm{nnnc} 2 \mathrm{c}([\mathrm{nH}] 1) \mathrm{cncc} 2$ & 3.20 & 2.28 & 9.54 & 10.75 & 17.48 & 19.59 & 0.57 & 0.50 \\
\hline S8169 & $\mathrm{O}=\mathrm{c} 1 \mathrm{nnn} 2 \mathrm{c}([\mathrm{nH}] 1) \mathrm{nccc} 2$ & 2.48 & 2.28 & 7.55 & 10.44 & 17.37 & 19.91 & 0.45 & 0.41 \\
\hline S8170 & $\mathrm{O}=\mathrm{c} 1 \mathrm{nnnc} 2 \mathrm{c}([\mathrm{nH}] 1) \mathrm{cccn} 2$ & 2.86 & 2.28 & 11.68 & 14.42 & 21.17 & 26.78 & 0.57 & 0.48 \\
\hline S8171 & $\mathrm{O}=\mathrm{c} 1[\mathrm{nH}] \mathrm{c} 2 \mathrm{ccncc} 2 \mathrm{c}(=\mathrm{O})[\mathrm{nH}] 1$ & 2.15 & 1.22 & 9.07 & 8.43 & 14.82 & 16.25 & 0.59 & 0.53 \\
\hline S8172 & $\mathrm{O}=\mathrm{c} 1[\mathrm{nH}] \mathrm{c} 2 \mathrm{cnccc} 2 \mathrm{c}(=\mathrm{O})[\mathrm{nH}] 1$ & 2.16 & 1.22 & 7.49 & 7.44 & 11.81 & 14.09 & 0.60 & 0.52 \\
\hline S8173 & $\mathrm{O}=\mathrm{c} 1[\mathrm{nH}] \mathrm{c} 2 \mathrm{ncccc} 2 \mathrm{c}(=\mathrm{O})[\mathrm{nH}] 1$ & 1.40 & 1.22 & 5.10 & 6.60 & 11.18 & 13.15 & 0.54 & 0.46 \\
\hline S8174 & $\mathrm{O}=\mathrm{c} 1[\mathrm{nH}] \mathrm{c} 2 \mathrm{cccnc} 2 \mathrm{c}(=\mathrm{O})[\mathrm{nH}] 1$ & 1.40 & 1.22 & 9.52 & 9.24 & 19.63 & 18.87 & 0.58 & 0.52 \\
\hline S8175 & $\mathrm{O}=\mathrm{c} 1[\mathrm{nH}] \mathrm{c} 2 \mathrm{cnccc} 2[\mathrm{nH}] \mathrm{c} 1=\mathrm{O}$ & 2.58 & 1.22 & 8.51 & 9.79 & 17.55 & 22.23 & 0.35 & 0.43 \\
\hline S8176 & $\mathrm{O}=\mathrm{c} 1[\mathrm{nH}] \mathrm{c} 2 \mathrm{ncccc} 2[\mathrm{nH}] \mathrm{c} 1=\mathrm{O}$ & 1.82 & 1.22 & 8.88 & 10.40 & 19.63 & 24.42 & 0.58 & 0.55 \\
\hline S8177 & $\mathrm{O}=\mathrm{c} 1 \mathrm{oc}(=\mathrm{O}) \mathrm{c} 2 \mathrm{c}([\mathrm{nH}] 1) \mathrm{ccnc} 2$ & 3.64 & 2.82 & 11.50 & 11.38 & 17.83 & 19.38 & 0.58 & 0.51 \\
\hline S8178 & $\mathrm{O}=\mathrm{c} 1 \mathrm{oc}(=\mathrm{O}) \mathrm{c} 2 \mathrm{c}([\mathrm{nH}] 1) \mathrm{cncc} 2$ & 3.64 & 2.82 & 9.98 & 9.98 & 15.90 & 16.65 & 0.24 & 0.29 \\
\hline S8179 & $\mathrm{O}=\mathrm{c} 1 \mathrm{oc}(=\mathrm{O}) \mathrm{c} 2 \mathrm{c}([\mathrm{nH}] 1) \mathrm{nccc} 2$ & 2.91 & 2.82 & 7.39 & 9.48 & 15.00 & 16.18 & 0.71 & 0.62 \\
\hline S8185 & $\mathrm{O}=\mathrm{c} 1 \mathrm{sc}(=\mathrm{O}) \mathrm{c} 2 \mathrm{c}([\mathrm{nH}] 1) \mathrm{ccnc} 2$ & 2.69 & 2.13 & 10.20 & 10.37 & 15.18 & 17.96 & 0.54 & 0.59 \\
\hline S8186 & $\mathrm{O}=\mathrm{c} 1 \mathrm{sc}(=\mathrm{O}) \mathrm{c} 2 \mathrm{c}([\mathrm{nH}] 1) \mathrm{cncc} 2$ & 2.70 & 2.13 & 8.50 & 9.00 & 13.03 & 15.30 & 0.56 & 0.61 \\
\hline S8187 & $\mathrm{O}=\mathrm{c} 1 \mathrm{sc}(=\mathrm{O}) \mathrm{c} 2 \mathrm{c}([\mathrm{nH}] 1) \mathrm{nccc} 2$ & 1.87 & 2.13 & 6.55 & 8.48 & 13.03 & 14.78 & 0.57 & 0.51 \\
\hline S8188 & $\mathrm{O}=\mathrm{c} 1 \mathrm{sc}(=\mathrm{O}) \mathrm{c} 2 \mathrm{c}([\mathrm{nH}] 1) \operatorname{ccc} 2$ & 1.89 & 2.13 & 10.60 & 12.14 & 19.41 & 21.84 & 0.82 & 0.75 \\
\hline S8189 & $\mathrm{O}=\mathrm{c} 1[\mathrm{nH}] \mathrm{c} 2 \mathrm{ccncc} 2 \mathrm{sc} 1=\mathrm{O}$ & 2.83 & 2.13 & 9.52 & 10.86 & 16.78 & 21.64 & 0.63 & 0.53 \\
\hline S8190 & $\mathrm{O}=\mathrm{c} 1[\mathrm{nH}] \mathrm{c} 2 \mathrm{cnccc} 2 \mathrm{sc} 1=\mathrm{O}$ & 2.80 & 2.13 & 7.73 & 9.85 & 14.87 & 20.30 & 0.68 & 0.64 \\
\hline S8191 & $\mathrm{O}=\mathrm{c} 1[\mathrm{nH}] \mathrm{c} 2 \mathrm{ncccc} 2 \mathrm{sc} 1=\mathrm{O}$ & 1.94 & 2.13 & 7.03 & 9.86 & 16.01 & 21.70 & 0.79 & 0.74 \\
\hline S8193 & $\mathrm{O}=\mathrm{c} 1 \mathrm{cc}[\mathrm{nH}] \mathrm{c} 2 \mathrm{c} 1 \mathrm{ccnc} 2$ & -1.15 & -1.38 & 10.48 & 7.58 & 21.64 & 18.73 & 0.83 & 0.75 \\
\hline S8194 & $\mathrm{O}=\mathrm{c} 1 \mathrm{cc}[\mathrm{nH}] \mathrm{c} 2 \mathrm{c} 1 \mathrm{cnc} 2$ & -1.13 & -1.38 & 11.36 & 9.17 & 23.55 & 22.19 & 0.65 & 0.55 \\
\hline S8195 & $\mathrm{O}=\mathrm{c} 1 \mathrm{cc}[\mathrm{nH}] \mathrm{c} 2 \mathrm{c} 1 \mathrm{nccc} 2$ & -1.86 & -1.38 & 10.85 & 10.49 & 27.71 & 25.28 & 0.84 & 0.84 \\
\hline S8196 & $\mathrm{O}=\mathrm{c} 1 \mathrm{cc}[\mathrm{nH}] \mathrm{c} 2 \mathrm{c} 1 \mathrm{ccc} 2$ & -1.89 & -1.38 & 6.33 & 6.25 & 18.04 & 16.10 & 0.30 & 0.35 \\
\hline
\end{tabular}




\begin{tabular}{|c|c|c|c|c|c|c|c|c|c|}
\hline S8197 & $\mathrm{O}=\mathrm{c} 1 \mathrm{nc}[\mathrm{nH}] \mathrm{c} 2 \mathrm{c} 1 \mathrm{ccnc} 2$ & 0.73 & 0.45 & 12.43 & 10.42 & 24.41 & 22.31 & 0.61 & 0.56 \\
\hline S8198 & $\mathrm{O}=\mathrm{c} 1 \mathrm{nc}[\mathrm{nH}] \mathrm{c} 2 \mathrm{c} 1 \mathrm{cncc} 2$ & 0.73 & 0.45 & 13.46 & 12.28 & 26.31 & 26.36 & 0.45 & 0.41 \\
\hline S8199 & $\mathrm{O}=\mathrm{c} 1 \mathrm{nc}[\mathrm{nH}] \mathrm{c} 2 \mathrm{c} 1 \mathrm{nccc} 2$ & 0.09 & 0.45 & 13.00 & 14.27 & 29.69 & 30.94 & 0.42 & 0.48 \\
\hline S82 & $\mathrm{O}=\mathrm{c} 1 \mathrm{cncn}[\mathrm{nH}] 1$ & 1.86 & 1.41 & 4.83 & 5.60 & 10.72 & 11.19 & 0.60 & 0.67 \\
\hline $\mathbf{S 8 2 0 0}$ & $\mathrm{O}=\mathrm{c} 1 \mathrm{nc}[\mathrm{nH}] \mathrm{c} 2 \mathrm{c} 1 \mathrm{ccc} 2$ & 0.03 & 0.45 & 8.27 & 9.32 & 21.04 & 20.18 & 0.61 & 0.53 \\
\hline S8201 & $\mathrm{n} 1 \mathrm{ccc} 2 \mathrm{c}(\mathrm{c} 1) \mathrm{nccn} 2$ & -0.61 & -0.32 & 4.62 & 4.61 & 10.43 & 11.02 & 0.67 & 0.71 \\
\hline $\mathbf{S 8 2 0 2}$ & $\operatorname{c} 1 \mathrm{ccc} 2 \mathrm{c}(\mathrm{n} 1) \operatorname{nccn} 2$ & -1.14 & -0.32 & 3.23 & 4.95 & 11.88 & 11.77 & 0.77 & 0.73 \\
\hline $\mathbf{S 8 2 0 3}$ & $\mathrm{n} 1 \mathrm{ccc} 2 \mathrm{c}(\mathrm{c} 1) \mathrm{ncnn} 2$ & 1.46 & 1.51 & 4.15 & 6.10 & 7.83 & 11.38 & 0.36 & 0.37 \\
\hline S8204 & $\mathrm{n} 1 \mathrm{ccc} 2 \mathrm{c}(\mathrm{c} 1) \mathrm{nncn} 2$ & 1.48 & 1.51 & 4.15 & 6.37 & 8.21 & 11.98 & 0.34 & 0.41 \\
\hline $\mathbf{S 8 2 0 7}$ & $\mathrm{O}=\mathrm{c} 1[\mathrm{nH}] \mathrm{cnc} 2 \mathrm{c} 1 \mathrm{ccnc} 2$ & 0.01 & 0.45 & 8.76 & 7.67 & 14.68 & 14.77 & 0.59 & 0.59 \\
\hline S8208 & $\mathrm{O}=\mathrm{c} 1[\mathrm{nH}] \mathrm{cnc} 2 \mathrm{c} 1 \mathrm{cncc} 2$ & -0.06 & 0.45 & 8.91 & 7.43 & 15.53 & 14.94 & 0.49 & 0.40 \\
\hline S8209 & $\mathrm{O}=\mathrm{c} 1[\mathrm{nH}] \mathrm{cnc} 2 \mathrm{c} 1 \mathrm{nccc} 2$ & -0.86 & 0.45 & 6.98 & 7.09 & 18.19 & 15.20 & 0.64 & 0.54 \\
\hline $\mathbf{S 8 2 1 0}$ & $\mathrm{O}=\mathrm{c} 1[\mathrm{nH}] \mathrm{cnc} 2 \mathrm{c} 1 \mathrm{ccc} 2$ & -0.49 & 0.45 & 7.24 & 7.71 & 15.45 & 14.76 & 0.38 & 0.41 \\
\hline S8211 & $\mathrm{O}=\mathrm{c} 1 \mathrm{cnc} 2 \mathrm{c}(\mathrm{o} 1) \mathrm{ccnc} 2$ & 2.26 & 2.05 & 6.64 & 6.76 & 11.19 & 12.62 & 0.58 & 0.65 \\
\hline $\mathbf{S 8 2 1 2}$ & $\mathrm{O}=\mathrm{c} 1 \mathrm{cnc} 2 \mathrm{c}(\mathrm{o} 1) \mathrm{cncc} 2$ & 2.26 & 2.05 & 6.88 & 6.62 & 11.72 & 12.15 & 0.54 & 0.49 \\
\hline S8215 & $\mathrm{O}=\mathrm{c} 1 \mathrm{ocnc} 2 \mathrm{c} 1 \mathrm{ccnc} 2$ & 1.71 & 2.05 & 6.01 & 6.62 & 10.56 & 12.07 & 0.43 & 0.54 \\
\hline S8216 & $\mathrm{O}=\mathrm{c} 1 \mathrm{ocnc} 2 \mathrm{c} 1 \mathrm{cncc} 2$ & 1.63 & 2.05 & 6.08 & 6.79 & 11.74 & 12.81 & 0.65 & 0.60 \\
\hline $\mathbf{S 8 2 1 7}$ & $\mathrm{O}=\mathrm{c} 1 \mathrm{ocnc} 2 \mathrm{c} 1 \mathrm{nccc} 2$ & 0.89 & 2.05 & 5.66 & 7.48 & 15.33 & 14.49 & 0.38 & 0.46 \\
\hline S8218 & $\mathrm{O}=\mathrm{c} 1 \mathrm{ocn} \operatorname{c} 2 \mathrm{c} 1 \mathrm{ccc} 2$ & 1.25 & 2.05 & 5.07 & 7.01 & 10.31 & 12.54 & 0.45 & 0.51 \\
\hline $\mathbf{S 8 2 2 2}$ & $\mathrm{O}=\mathrm{c} 1 \mathrm{cnc} 2 \mathrm{c}(\mathrm{s} 1) \operatorname{ccc} 2$ & 1.00 & 1.36 & 6.36 & 6.93 & 12.06 & 14.31 & 0.35 & 0.41 \\
\hline S8226 & $\mathrm{O}=\mathrm{c} 1 \mathrm{scn} \operatorname{c} 2 \mathrm{c} 1 \mathrm{ccc} 2$ & 0.26 & 1.36 & 4.21 & 6.24 & 10.26 & 11.77 & 0.73 & 0.71 \\
\hline S8227 & $\mathrm{n} 1 \mathrm{ccc} 2 \mathrm{c}(\mathrm{c} 1) \mathrm{nnnn} 2$ & 3.68 & 3.34 & 8.02 & 8.88 & 13.19 & 14.58 & 0.61 & 0.51 \\
\hline $\mathbf{S 8 2 2 8}$ & c1ccc $2 \mathrm{c}(\mathrm{n} 1) \mathrm{nnnn} 2$ & 3.34 & 3.34 & 10.32 & 10.13 & 17.99 & 17.32 & 0.55 & 0.47 \\
\hline S8229 & $\mathrm{O}=\mathrm{c} 1[\mathrm{nH}][\mathrm{n}] \mathrm{c} 2 \mathrm{c}([\mathrm{n}] 1) \mathrm{ccnc} 2$ & 2.35 & 2.28 & 7.57 & 7.83 & 16.66 & 13.98 & 0.77 & 0.63 \\
\hline $\mathbf{S 8 2 3}$ & {$[\mathrm{nH}] 1 \mathrm{cc} 2 \mathrm{c}(\mathrm{c} 1) \operatorname{ccs} 2$} & -6.33 & -5.66 & 4.23 & 2.52 & 16.34 & 13.32 & 0.35 & 0.39 \\
\hline $\mathbf{S 8 2 3 0}$ & $\mathrm{O}=\mathrm{c} 1[\mathrm{nH}][\mathrm{n}] \mathrm{c} 2 \mathrm{c}([\mathrm{n}] 1) \mathrm{cncc} 2$ & 2.44 & 2.28 & 7.35 & 8.43 & 16.39 & 15.28 & 0.38 & 0.46 \\
\hline S824 & $\mathrm{o} 1 \mathrm{cc} 2 \mathrm{c}(\mathrm{c} 1) \operatorname{ccs} 2$ & -4.15 & -4.06 & 0.27 & 1.41 & 7.60 & 10.00 & 0.38 & 0.39 \\
\hline S8241 & $\mathrm{O}=\mathrm{c} 1[\mathrm{nH}] \mathrm{nnc} 2 \mathrm{c} 1 \mathrm{cncc} 2$ & 1.82 & 2.28 & 5.49 & 7.72 & 9.88 & 13.01 & 0.77 & 0.70 \\
\hline $\mathbf{S 8 2 4 2}$ & $\mathrm{O}=\mathrm{c} 1[\mathrm{nH}] \mathrm{nnc} 2 \mathrm{c} 1 \mathrm{ccnc} 2$ & 1.91 & 2.28 & 5.58 & 8.22 & 8.95 & 13.44 & 0.36 & 0.43 \\
\hline S8244 & $\mathrm{O}=\mathrm{c} 1[\mathrm{nH}] \mathrm{nnc} 2 \mathrm{c} 1 \mathrm{nccc} 2$ & 1.10 & 2.28 & 6.36 & 7.61 & 15.27 & 13.76 & 0.68 & 0.63 \\
\hline S825 & $\mathrm{c} 1 \mathrm{cc} 2 \mathrm{c}(\mathrm{s} 1) \operatorname{scc} 2$ & -4.59 & -4.75 & -0.12 & 0.77 & 7.50 & 9.39 & 0.55 & 0.55 \\
\hline
\end{tabular}




\begin{tabular}{|c|c|c|c|c|c|c|c|c|c|}
\hline S8255 & $\mathrm{O}=\mathrm{c} 1 \mathrm{sn}[\mathrm{n}] \mathrm{c} 2 \mathrm{c} 1 \mathrm{ccc} 2$ & 2.21 & 3.19 & 7.56 & 8.91 & 12.65 & 13.82 & 0.51 & 0.44 \\
\hline S826 & $\mathrm{c} 1 \mathrm{cc} 2 \mathrm{c}(\mathrm{s} 1) \operatorname{ccs} 2$ & -4.58 & -4.75 & -0.17 & 0.62 & 7.36 & 9.37 & 0.33 & 0.43 \\
\hline S8261 & $\mathrm{O}=\mathrm{c} 1 \mathrm{c} \operatorname{coc} 2 \mathrm{c} 1 \mathrm{ccn} 22$ & 0.09 & 0.22 & 6.21 & 5.46 & 13.63 & 13.22 & 0.59 & 0.56 \\
\hline S8262 & $\mathrm{O}=\mathrm{c} 1 \mathrm{c} \operatorname{coc} 2 \mathrm{c} 1 \mathrm{cnc} 2$ & 0.05 & 0.22 & 6.51 & 5.97 & 15.15 & 15.16 & 0.61 & 0.61 \\
\hline S8263 & $\mathrm{O}=\mathrm{c} 1 \mathrm{c} \operatorname{coc} 2 \mathrm{c} 1 \mathrm{nccc} 2$ & -0.73 & 0.22 & 5.64 & 6.64 & 18.87 & 17.35 & 0.51 & 0.65 \\
\hline S8264 & $\mathrm{O}=\mathrm{c} 1 \mathrm{c} \operatorname{coc} 2 \mathrm{c} 1 \mathrm{ccc} 2$ & -0.45 & 0.22 & 3.77 & 5.29 & 11.76 & 12.21 & 0.61 & 0.69 \\
\hline S8265 & $\mathrm{O}=\mathrm{c} 1 \mathrm{ncoc} 2 \mathrm{c} 1 \mathrm{ccnc} 2$ & 1.93 & 2.05 & 7.65 & 7.41 & 15.87 & 15.55 & 0.43 & 0.48 \\
\hline S8266 & $\mathrm{O}=\mathrm{c} 1 \mathrm{ncoc} 2 \mathrm{c} 1 \mathrm{cncc} 2$ & 1.89 & 2.05 & 8.12 & 8.19 & 17.35 & 18.08 & 0.78 & 0.66 \\
\hline S8267 & $\mathrm{O}=\mathrm{c} 1 \mathrm{n} \operatorname{coc} 2 \mathrm{c} 1 \mathrm{n} \operatorname{ccc} 2$ & 1.20 & 2.05 & 8.04 & 9.54 & 20.99 & 21.77 & 0.43 & 0.48 \\
\hline S8268 & $\mathrm{O}=\mathrm{c} 1 \mathrm{n} \operatorname{coc} 2 \mathrm{c} 1 \mathrm{ccc} 2$ & 1.45 & 2.05 & 5.63 & 7.47 & 14.69 & 15.04 & 0.79 & 0.67 \\
\hline S827 & $\operatorname{s1cc} 2 \mathrm{c}(\mathrm{c} 1) \operatorname{ccs} 2$ & -4.57 & -4.75 & 0.05 & 0.67 & 7.67 & 9.38 & 0.71 & 0.59 \\
\hline S8273 & $\mathrm{O}=\mathrm{c} 1 \mathrm{cn}[\mathrm{nH}] \mathrm{c} 2 \mathrm{c} 1 \mathrm{ccnc} 2$ & 0.65 & 0.45 & 8.57 & 7.11 & 16.51 & 15.15 & 0.58 & 0.53 \\
\hline S8274 & $\mathrm{O}=\mathrm{c} 1 \mathrm{cn}[\mathrm{nH}] \mathrm{c} 2 \mathrm{c} 1 \mathrm{cncc} 2$ & 0.64 & 0.45 & 9.66 & 8.44 & 18.47 & 18.01 & 0.58 & 0.52 \\
\hline S8276 & $\mathrm{O}=\mathrm{c} 1 \mathrm{cn}[\mathrm{nH}] \mathrm{c} 2 \mathrm{c} 1 \mathrm{ccc} 2$ & -0.01 & 0.45 & 4.23 & 6.46 & 12.70 & 14.00 & 0.40 & 0.48 \\
\hline S828 & $\mathrm{c} 1 \mathrm{cc} 2 \mathrm{c}(\mathrm{s} 1)[\mathrm{nH}] \mathrm{nc} 2$ & -3.80 & -3.83 & 2.48 & 2.99 & 10.32 & 12.74 & 0.81 & 0.74 \\
\hline $\mathbf{S 8 2 8 3}$ & $\mathrm{O}=\mathrm{c} 1 \mathrm{cnsc} 2 \mathrm{c} 1 \mathrm{nccc} 2$ & 0.57 & 1.36 & 7.09 & 7.01 & 17.18 & 15.51 & 0.82 & 0.77 \\
\hline S8289 & $\mathrm{O}=\mathrm{c} 1 \mathrm{nn}[\mathrm{nH}] \mathrm{c} 2 \mathrm{c} 1 \mathrm{ccnc} 2$ & 2.52 & 2.28 & 11.24 & 10.97 & 20.75 & 20.97 & 0.64 & 0.53 \\
\hline S829 & $\mathrm{c} 1 \mathrm{sc} 2 \mathrm{c}(\mathrm{c} 1)[\mathrm{nH}] \mathrm{nc} 2$ & -3.75 & -3.83 & 3.18 & 3.55 & 11.18 & 12.91 & 0.81 & 0.78 \\
\hline $\mathbf{S 8 2 9 0}$ & $\mathrm{O}=\mathrm{c} 1 \mathrm{nn}[\mathrm{nH}] \mathrm{c} 2 \mathrm{c} 1 \mathrm{cncc} 2$ & 2.50 & 2.28 & 12.35 & 12.57 & 22.44 & 24.43 & 0.73 & 0.60 \\
\hline S8292 & $\mathrm{O}=\mathrm{c} 1 \mathrm{nn}[\mathrm{nH}] \mathrm{c} 2 \mathrm{c} 1 \mathrm{ccc} 2$ & 1.90 & 2.28 & 7.00 & 10.55 & 17.35 & 20.33 & 0.45 & 0.56 \\
\hline S83 & $\mathrm{O}=\mathrm{c} \operatorname{lncnc}[\mathrm{nH}] 1$ & 2.03 & 1.41 & 11.12 & 8.21 & 20.61 & 16.87 & 0.53 & 0.45 \\
\hline S830 & $\mathrm{c} 1 \mathrm{cc} 2 \mathrm{c}(\mathrm{s} 1) \mathrm{n}[\mathrm{nH}] \mathrm{c} 2$ & -4.53 & -3.83 & 4.09 & 3.69 & 13.82 & 12.99 & 0.35 & 0.42 \\
\hline S8303 & $\mathrm{O}=\mathrm{c} 1 \mathrm{oc} 2 \mathrm{cnccc} 2 \mathrm{c}(=\mathrm{O})[\mathrm{nH}] 1$ & 3.18 & 2.82 & 8.47 & 8.01 & 12.77 & 12.59 & 0.57 & 0.51 \\
\hline S8304 & $\mathrm{O}=\mathrm{c} 1 \mathrm{oc} 2 \mathrm{ccncc} 2 \mathrm{c}(=\mathrm{O})[\mathrm{nH}] 1$ & 3.12 & 2.82 & 8.29 & 7.92 & 12.13 & 13.23 & 0.61 & 0.53 \\
\hline S8305 & $\mathrm{O}=\mathrm{c} 1 \mathrm{oc} 2 \operatorname{ccc} n \mathrm{c} 2 \mathrm{c}(=\mathrm{O})[\mathrm{nH}] 1$ & 2.36 & 2.82 & 7.51 & 8.09 & 15.03 & 14.96 & 0.32 & 0.41 \\
\hline S8306 & $\mathrm{O}=\mathrm{c} 1 \mathrm{oc} 2 \mathrm{ncccc} 2 \mathrm{c}(=\mathrm{O})[\mathrm{nH}] 1$ & 2.63 & 2.82 & 6.73 & 8.34 & 13.56 & 13.27 & 0.63 & 0.51 \\
\hline S8307 & $\mathrm{O}=\mathrm{c} 1 \mathrm{sc} 2 \mathrm{cnccc} 2 \mathrm{c}(=\mathrm{O})[\mathrm{nH}] 1$ & 2.44 & 2.13 & 6.74 & 7.16 & 10.87 & 11.74 & 0.35 & 0.40 \\
\hline S8308 & $\mathrm{O}=\mathrm{c} 1 \mathrm{sc} 2 \mathrm{ccncc} 2 \mathrm{c}(=\mathrm{O})[\mathrm{nH}] 1$ & 2.40 & 2.13 & 6.43 & 7.14 & 10.27 & 12.57 & 0.35 & 0.43 \\
\hline S8309 & $\mathrm{O}=\mathrm{c} 1 \mathrm{sc} 2 \mathrm{cccnc} 2 \mathrm{c}(=\mathrm{O})[\mathrm{nH}] 1$ & 1.56 & 2.13 & 7.63 & 7.35 & 15.68 & 14.40 & 0.66 & 0.57 \\
\hline S831 & $\mathrm{c} 1 \mathrm{cc} 2 \mathrm{c}(\mathrm{s} 1) \mathrm{c}[\mathrm{nH}] \mathrm{n} 2$ & -4.60 & -3.83 & 3.96 & 3.19 & 12.51 & 12.78 & 0.44 & 0.44 \\
\hline S832 & $\mathrm{c} 1 \mathrm{cc} 2 \mathrm{c}(\mathrm{s} 1) \mathrm{onc} 2$ & -1.76 & -2.23 & 3.28 & 3.34 & 11.23 & 10.89 & 0.58 & 0.61 \\
\hline
\end{tabular}




\begin{tabular}{|c|c|c|c|c|c|c|c|c|c|}
\hline S833 & $\mathrm{c} 1 \mathrm{cc} 2 \mathrm{c}(\mathrm{s} 1) \mathrm{cno} 2$ & -1.82 & -2.23 & 3.43 & 3.14 & 11.50 & 10.85 & 0.39 & 0.46 \\
\hline S8330 & $\mathrm{O}=\mathrm{c} 1 \mathrm{oc} 2 \mathrm{ncccc} 2 \mathrm{c}(=\mathrm{O}) \mathrm{s} 1$ & 2.92 & 3.73 & 7.71 & 8.95 & 14.26 & 13.85 & 0.58 & 0.51 \\
\hline S8332 & $\mathrm{O}=\mathrm{c} 1 \mathrm{sc} 2 \mathrm{c}(\mathrm{s} 1) \operatorname{ccc} 2$ & 0.18 & 0.06 & 6.01 & 5.76 & 13.08 & 13.36 & 0.40 & 0.47 \\
\hline S834 & c1cc2c(s1)noc2 & -2.02 & -2.23 & 3.78 & 3.54 & 12.52 & 11.01 & 0.57 & 0.46 \\
\hline S8343 & $\mathrm{c} 1 \mathrm{nnnc} 2 \mathrm{c}(\mathrm{c} 1) \mathrm{c}[\mathrm{nH}] \mathrm{c} 2$ & -3.17 & -2.53 & 8.66 & 7.32 & 22.15 & 18.70 & 0.54 & 0.58 \\
\hline S8344 & $\mathrm{c} 1 \mathrm{nnn} 2 \mathrm{c}(\mathrm{c} 1) \operatorname{coc} 2$ & -1.08 & -0.93 & 4.66 & 5.51 & 14.08 & 14.27 & 0.53 & 0.48 \\
\hline S8345 & $\mathrm{c} 1 \mathrm{nnn} 2 \mathrm{c}(\mathrm{c} 1) \csc 2$ & -1.59 & -1.62 & 4.53 & 4.81 & 14.72 & 13.78 & 0.59 & 0.68 \\
\hline S8346 & $\mathrm{c} 1 \mathrm{nnc} 2 \mathrm{c}(\mathrm{c} 1) \mathrm{n}[\mathrm{nH}] \mathrm{c} 2$ & -1.54 & -0.70 & 8.48 & 6.97 & 18.73 & 15.47 & 0.75 & 0.64 \\
\hline S8347 & $\mathrm{c} 1 \mathrm{nnc} 2 \mathrm{c}(\mathrm{c} 1) \mathrm{c}[\mathrm{nH}] \mathrm{n} 2$ & -1.25 & -0.70 & 9.05 & 9.47 & 21.97 & 20.96 & 0.42 & 0.49 \\
\hline S8349 & $\mathrm{c} 1 \mathrm{nnc} 2 \mathrm{c}(\mathrm{c} 1) \operatorname{con} 2$ & 1.17 & 0.90 & 9.04 & 8.61 & 18.07 & 17.88 & 0.34 & 0.42 \\
\hline S835 & $\mathrm{c} 1 \mathrm{cc} 2 \mathrm{c}(\mathrm{s} 1) \operatorname{con} 2$ & -2.09 & -2.23 & 3.09 & 3.04 & 11.42 & 10.80 & 0.58 & 0.63 \\
\hline S8351 & $\mathrm{c} 1 \mathrm{nnn} 2 \mathrm{c}(\mathrm{c} 1) \operatorname{csn} 2$ & 0.30 & 0.21 & 8.25 & 7.85 & 19.00 & 17.22 & 0.65 & 0.67 \\
\hline S8352 & $\mathrm{c} 1 \mathrm{nnc} 2 \mathrm{c}(\mathrm{c} 1) \mathrm{n}[\mathrm{nH}] \mathrm{n} 2$ & 0.39 & 1.13 & 7.45 & 8.09 & 16.57 & 15.49 & 0.83 & 0.83 \\
\hline S8355 & $\mathrm{c} 1 \mathrm{nnc} 2 \mathrm{c}(\mathrm{c} 1) \mathrm{ccnn} 2$ & 1.81 & 1.51 & 10.07 & 10.87 & 19.82 & 21.87 & 0.33 & 0.33 \\
\hline S8357 & n1ncc2c(c1)nncc2 & 2.39 & 1.51 & 9.53 & 8.44 & 16.48 & 16.53 & 0.65 & 0.66 \\
\hline S8358 & $\mathrm{O}=\mathrm{c} 1[\mathrm{nH}] \mathrm{ccc} 2 \mathrm{c} 1 \mathrm{nncc} 2$ & -0.01 & 0.45 & 8.84 & 10.09 & 22.01 & 22.93 & 0.56 & 0.48 \\
\hline S8359 & $\mathrm{O}=\mathrm{c} 1[\mathrm{nH}] \mathrm{ccc} 2 \mathrm{c} 1 \mathrm{ccnn} 2$ & 0.15 & 0.45 & 8.06 & 8.31 & 16.21 & 16.55 & 0.40 & 0.50 \\
\hline S836 & $\mathrm{c} 1 \mathrm{cc} 2 \mathrm{c}(\mathrm{s} 1) \mathrm{snc} 2$ & -2.47 & -2.92 & 2.69 & 2.50 & 9.84 & 10.08 & 0.59 & 0.49 \\
\hline S8365 & $\mathrm{O}=\mathrm{c} 1 \mathrm{occc} 2 \mathrm{c} 1 \mathrm{ccnn} 2$ & 1.85 & 2.05 & 5.37 & 7.64 & 10.69 & 14.38 & 0.44 & 0.44 \\
\hline S8366 & $\mathrm{O}=\mathrm{c} 1 \mathrm{ccc} 2 \mathrm{c}(\mathrm{o} 1) \operatorname{ccn} n 2$ & 2.35 & 2.05 & 6.19 & 7.78 & 11.73 & 14.91 & 0.24 & 0.30 \\
\hline S837 & $\mathrm{c} 1 \mathrm{sc} 2 \mathrm{c}(\mathrm{c} 1) \mathrm{snc} 2$ & -2.46 & -2.92 & 3.00 & 2.35 & 10.39 & 10.07 & 0.62 & 0.75 \\
\hline S8376 & $\mathrm{c} 1 \mathrm{nnc} 2 \mathrm{c}(\mathrm{c} 1) \mathrm{cncn} 2$ & 1.79 & 1.51 & 9.14 & 8.27 & 17.96 & 16.15 & 0.57 & 0.51 \\
\hline S8377 & $\mathrm{n} 1 \mathrm{cnc} 2 \mathrm{c}(\mathrm{c} 1) \mathrm{nncc} 2$ & 1.45 & 1.51 & 4.78 & 6.63 & 9.96 & 12.55 & 0.59 & 0.47 \\
\hline S8378 & $\mathrm{c} 1 \mathrm{nnc} 2 \mathrm{c}(\mathrm{c} 1) \mathrm{cnnn} 2$ & 4.03 & 3.34 & 12.73 & 12.02 & 20.14 & 21.49 & 0.59 & 0.62 \\
\hline S838 & $\mathrm{c} 1 \mathrm{cc} 2 \mathrm{c}(\mathrm{s} 1) \mathrm{nsc} 2$ & -2.85 & -2.92 & 3.21 & 2.73 & 11.03 & 10.22 & 0.61 & 0.51 \\
\hline S8380 & $\mathrm{O}=\mathrm{c} 1[\mathrm{nH}] \mathrm{ncc} 2 \mathrm{c} 1 \mathrm{nncc} 2$ & 1.95 & 2.28 & 9.73 & 9.01 & 20.67 & 18.01 & 0.62 & 0.55 \\
\hline S8381 & $\mathrm{O}=\mathrm{c} 1[\mathrm{nH}] \mathrm{ncc} 2 \mathrm{c} 1 \mathrm{ccnn} 2$ & 2.13 & 2.28 & 5.71 & 8.22 & 10.18 & 13.80 & 0.64 & 0.55 \\
\hline S839 & $\mathrm{c} 1 \mathrm{cc} 2 \mathrm{c}(\mathrm{s} 1) \operatorname{csn} 2$ & -2.93 & -2.92 & 2.33 & 2.23 & 9.52 & 10.01 & 0.30 & 0.30 \\
\hline S840 & $\mathrm{c} 1 \mathrm{cc} 2 \mathrm{c}(\mathrm{s} 1)[\mathrm{nH}] \mathrm{cn} 2$ & -4.18 & -3.83 & 6.25 & 4.67 & 16.50 & 15.53 & 0.43 & 0.50 \\
\hline S8405 & $\mathrm{O}=\mathrm{c} 1 \mathrm{cnc} 2 \mathrm{c}([\mathrm{nH}] 1) \mathrm{nnncc} 2$ & 2.66 & 2.28 & 6.98 & 7.91 & 11.76 & 14.18 & 0.44 & 0.53 \\
\hline S8407 & $\mathrm{O}=\mathrm{c} 1 \mathrm{nnc} 2 \mathrm{c}([\mathrm{nH}] 1) \mathrm{nncc} 2$ & 4.44 & 4.11 & 9.87 & 11.43 & 16.46 & 19.26 & 0.62 & 0.69 \\
\hline
\end{tabular}




\begin{tabular}{|c|c|c|c|c|c|c|c|c|c|}
\hline S8409 & $\mathrm{O}=\mathrm{c} 1[\mathrm{nH}] \mathrm{c} 2 \mathrm{nnccc} 2 \mathrm{c}(=\mathrm{O})[\mathrm{nH}] 1$ & 3.54 & 3.05 & 8.69 & 8.76 & 12.38 & 14.42 & 0.42 & 0.37 \\
\hline S841 & $\mathrm{c} 1 \mathrm{sc} 2 \mathrm{c}(\mathrm{c} 1)[\mathrm{nH}] \mathrm{cn} 2$ & -4.05 & -3.83 & 6.75 & 5.73 & 17.46 & 15.91 & 0.69 & 0.75 \\
\hline S8416 & $\mathrm{O}=\mathrm{c} 1 \mathrm{sc}(=\mathrm{O}) \mathrm{c} 2 \mathrm{c}([\mathrm{nH}] 1) \mathrm{nncc} 2$ & 3.95 & 3.96 & 9.31 & 9.84 & 13.41 & 15.00 & 0.68 & 0.60 \\
\hline S8419 & $\mathrm{O}=\mathrm{c} 1 \mathrm{cc}[\mathrm{nH}] \mathrm{c} 2 \mathrm{c} 1 \mathrm{ccnn} 2$ & 0.25 & 0.45 & 8.61 & 8.10 & 16.85 & 17.30 & 0.77 & 0.66 \\
\hline S842 & $\mathrm{c} 1 \mathrm{nc} 2 \mathrm{c}(\mathrm{o} 1) \mathrm{scc} 2$ & -2.41 & -2.23 & 1.56 & 2.52 & 7.63 & 10.19 & 0.48 & 0.45 \\
\hline S8421 & $\mathrm{O}=\mathrm{c} 1 \mathrm{nc}[\mathrm{nH}] \mathrm{c} 2 \mathrm{c} 1 \mathrm{ccnn} 2$ & 2.16 & 2.28 & 10.31 & 10.60 & 19.59 & 20.13 & 0.58 & 0.47 \\
\hline S8423 & $\mathrm{c} 1 \mathrm{cnc} 2 \mathrm{c}(\mathrm{n} 1) \mathrm{nncc} 2$ & 0.96 & 1.51 & 6.61 & 7.64 & 16.01 & 14.77 & 0.68 & 0.60 \\
\hline S8426 & $\mathrm{O}=\mathrm{c} 1[\mathrm{nH}] \mathrm{cnc} 2 \mathrm{c} 1 \mathrm{ccnn} 2$ & 1.75 & 2.28 & 10.43 & 11.00 & 19.91 & 18.43 & 0.75 & 0.77 \\
\hline S8427 & $\mathrm{O}=\mathrm{c} 1[\mathrm{nH}] \mathrm{cnc} 2 \mathrm{c} 1 \mathrm{nncc} 2$ & 1.25 & 2.28 & 10.42 & 10.15 & 21.06 & 19.04 & 0.32 & 0.39 \\
\hline S843 & $\mathrm{c} 1 \mathrm{oc} 2 \mathrm{c}(\mathrm{n} 1) \operatorname{scc} 2$ & -2.34 & -2.23 & 1.62 & 2.83 & 8.05 & 10.36 & 0.51 & 0.45 \\
\hline S8430 & $\mathrm{O}=\mathrm{c} 1 \mathrm{ocn} \operatorname{2c} 1 \mathrm{ccnn} 2$ & 3.46 & 3.88 & 8.78 & 9.44 & 15.25 & 15.02 & 0.74 & 0.68 \\
\hline S844 & c1sc2c(c1)ncs2 & -3.12 & -2.92 & 0.96 & 1.85 & 7.46 & 9.81 & 0.32 & 0.39 \\
\hline S845 & $\mathrm{c} 1 \mathrm{sc} 2 \mathrm{c}(\mathrm{c} 1) \operatorname{sen} 2$ & -3.02 & -2.92 & 1.55 & 2.20 & 8.47 & 10.01 & 0.46 & 0.60 \\
\hline S8454 & $\mathrm{O}=\mathrm{c} 1 \mathrm{c} \operatorname{coc} 2 \mathrm{c} 1 \mathrm{ccn} n 2$ & 1.78 & 2.05 & 6.45 & 7.72 & 12.41 & 14.21 & 0.63 & 0.61 \\
\hline S8456 & $\mathrm{O}=\mathrm{c} 1 \mathrm{n} \operatorname{coc} 2 \mathrm{c} 1 \mathrm{ccnn} 2$ & 3.64 & 3.88 & 8.81 & 9.33 & 14.30 & 15.80 & 0.32 & 0.40 \\
\hline S846 & $\mathrm{c} 1 \mathrm{cc} 2 \mathrm{c}(\mathrm{s} 1)[\mathrm{nH}] \mathrm{nn} 2$ & -2.01 & -2.00 & 5.78 & 6.15 & 15.85 & 16.45 & 0.60 & 0.59 \\
\hline S8460 & $\mathrm{O}=\mathrm{c} 1 \mathrm{cn}[\mathrm{nH}] \mathrm{c} 2 \mathrm{c} 1 \mathrm{ccnn} 2$ & 2.09 & 2.28 & 5.91 & 8.02 & 10.91 & 14.55 & 0.42 & 0.37 \\
\hline S8468 & $\mathrm{O}=\mathrm{c} 1 \mathrm{nn}[\mathrm{nH}] \mathrm{c} 2 \mathrm{c} 1 \mathrm{ccnn} 2$ & 3.99 & 4.11 & 9.26 & 11.54 & 15.97 & 19.64 & 0.35 & 0.46 \\
\hline S847 & $\mathrm{c} 1 \mathrm{sc} 2 \mathrm{c}(\mathrm{c} 1)[\mathrm{nH}] \mathrm{nn} 2$ & -1.86 & -2.00 & 6.39 & 7.21 & 16.84 & 16.83 & 0.28 & 0.37 \\
\hline S8472 & $\mathrm{O}=\mathrm{c} 1 \mathrm{ccc}(=\mathrm{O}) \mathrm{n} 2 \mathrm{n} 1 \mathrm{cccc} 2$ & 0.36 & 0.89 & 3.83 & 6.12 & 9.96 & 13.76 & 0.52 & 0.45 \\
\hline S848 & {$[\mathrm{nH}] 1 \mathrm{nc} 2 \mathrm{c}(\mathrm{n} 1) \mathrm{ccs} 2$} & -2.76 & -2.00 & 2.87 & 3.34 & 8.02 & 10.20 & 0.50 & 0.40 \\
\hline S849 & c1cc2c(s1)onn2 & -0.15 & -0.40 & 5.01 & 4.95 & 11.54 & 12.44 & 0.65 & 0.57 \\
\hline S850 & c1cc2c(s1)nno2 & -0.05 & -0.40 & 5.71 & 5.25 & 12.75 & 12.61 & 0.47 & 0.52 \\
\hline S851 & $\mathrm{c} 1 \mathrm{cc} 2 \mathrm{c}(\mathrm{s} 1) \operatorname{snn} 2$ & -0.94 & -1.09 & 4.30 & 4.21 & 11.95 & 11.89 & 0.60 & 0.53 \\
\hline S852 & c1cc2c(s1)nns2 & -0.83 & -1.09 & 4.95 & 4.57 & 13.02 & 12.09 & 0.47 & 0.61 \\
\hline S8528 & {$[\mathrm{nH}] 1 \mathrm{cc} 2 \mathrm{c}(\mathrm{c} 1) \mathrm{cnnc} 2$} & -2.47 & -2.53 & 10.45 & 7.92 & 25.63 & 19.62 & 0.82 & 0.73 \\
\hline S8529 & o1cc2c(c1)cnnc2 & -0.38 & -0.93 & 5.18 & 5.30 & 16.31 & 14.07 & 0.41 & 0.47 \\
\hline S853 & $\mathrm{O}=\mathrm{c} 1 \mathrm{c}(=\mathrm{O}) \mathrm{c} 2 \mathrm{c}(\mathrm{c} 1=\mathrm{O}) \mathrm{scc} 2$ & 3.74 & 4.62 & 9.85 & 10.06 & 16.80 & 20.55 & 0.84 & 0.94 \\
\hline $\mathbf{S 8 5 3 0}$ & $\operatorname{s} 1 \mathrm{cc} 2 \mathrm{c}(\mathrm{c} 1) \mathrm{cnn} \mathrm{c} 2$ & -0.77 & -1.62 & 4.96 & 4.66 & 16.65 & 13.71 & 0.40 & 0.32 \\
\hline S8531 & $\mathrm{n} 1 \mathrm{ncc} 2 \mathrm{c}(\mathrm{c} 1) \mathrm{n}[\mathrm{nH}] \mathrm{c} 2$ & -0.85 & -0.70 & 10.52 & 8.48 & 22.95 & 18.41 & 0.52 & 0.50 \\
\hline S8532 & $\mathrm{n} 1 \mathrm{ncc} 2 \mathrm{c}(\mathrm{c} 1) \operatorname{noc} 2$ & 1.56 & 0.90 & 9.06 & 6.82 & 16.87 & 14.18 & 0.52 & 0.62 \\
\hline
\end{tabular}




\begin{tabular}{|c|c|c|c|c|c|c|c|c|c|}
\hline S8533 & $\mathrm{n} 1 \mathrm{ncc} 2 \mathrm{c}(\mathrm{c} 1) \mathrm{nsc} 2$ & 0.80 & 0.21 & 8.40 & 6.11 & 18.10 & 13.67 & 0.33 & 0.43 \\
\hline S8534 & $\mathrm{n} 1 \mathrm{ncc} 2 \mathrm{c}(\mathrm{c} 1) \mathrm{n}[\mathrm{nH}] \mathrm{n} 2$ & 0.78 & 1.13 & 9.15 & 8.02 & 18.44 & 14.94 & 0.33 & 0.43 \\
\hline S8537 & $\mathrm{n} 1 \mathrm{ncc} 2 \mathrm{c}(\mathrm{c} 1) \mathrm{cnnc} 2$ & 3.17 & 1.51 & 7.26 & 7.54 & 11.78 & 14.54 & 0.57 & 0.49 \\
\hline S8538 & $\mathrm{O}=\mathrm{c} 1[\mathrm{nH}] \mathrm{ccc} 2 \mathrm{c} 1 \mathrm{cnnc} 2$ & 0.97 & 0.45 & 10.47 & 9.52 & 20.78 & 19.99 & 0.47 & 0.58 \\
\hline S854 & $\mathrm{o} 1 \mathrm{nc} 2 \mathrm{c}(\mathrm{n} 1) \operatorname{ccs} 2$ & 0.08 & -0.40 & 6.46 & 4.14 & 12.80 & 9.55 & 0.35 & 0.35 \\
\hline S8541 & $\mathrm{O}=\mathrm{c} 1 \mathrm{occc} 2 \mathrm{c} 1 \mathrm{cnn} \mathrm{c} 2$ & 2.63 & 2.05 & 8.84 & 8.92 & 16.12 & 17.92 & 0.50 & 0.44 \\
\hline S8542 & $\mathrm{O}=\mathrm{c} 1 \mathrm{ccc} 2 \mathrm{c}(\mathrm{o} 1) \mathrm{cnnc} 2$ & 3.29 & 2.05 & 9.85 & 8.28 & 16.23 & 15.48 & 0.69 & 0.59 \\
\hline S8547 & c1ncc $2 \mathrm{c}(\mathrm{n} 1) \mathrm{cnnc} 2$ & 2.38 & 1.51 & 7.62 & 6.83 & 14.61 & 12.98 & 0.77 & 0.80 \\
\hline S8548 & n1ncc $2 \mathrm{c}(\mathrm{c} 1) \mathrm{nnnc} 2$ & 4.52 & 3.34 & 11.22 & 9.64 & 15.26 & 16.24 & 0.48 & 0.39 \\
\hline S8549 & $\mathrm{O}=\mathrm{c} 1[\mathrm{nH}] \mathrm{ncc} 2 \mathrm{c} 1 \mathrm{cnnc} 2$ & 2.88 & 2.28 & 8.21 & 8.49 & 14.62 & 15.17 & 1.07 & 0.95 \\
\hline S855 & $\operatorname{s} 1 \mathrm{nc} 2 \mathrm{c}(\mathrm{n} 1) \operatorname{ccs} 2$ & -1.13 & -1.09 & 3.80 & 3.28 & 7.94 & 8.60 & 0.34 & 0.42 \\
\hline S856 & $\mathrm{O}=\mathrm{c} 1[\mathrm{nH}] \mathrm{c} 2 \mathrm{c}([\mathrm{nH}] 1) \operatorname{ccs} 2$ & -2.11 & -3.06 & 2.98 & 4.28 & 13.38 & 14.87 & 0.37 & 0.44 \\
\hline S8561 & $\mathrm{O}=\mathrm{c} 1 \mathrm{cnc} 2 \mathrm{c}([\mathrm{nH}] 1) \mathrm{cnnc} 2$ & 3.66 & 2.28 & 10.32 & 9.67 & 16.98 & 16.35 & 0.32 & 0.32 \\
\hline S8562 & $\mathrm{O}=\mathrm{c} 1 \mathrm{nnc} 2 \mathrm{c}([\mathrm{nH}] 1) \mathrm{cnn} c 2$ & 5.43 & 4.11 & 13.65 & 13.24 & 18.26 & 21.53 & 0.59 & 0.46 \\
\hline S8563 & $\mathrm{O}=\mathrm{c} 1[\mathrm{nH}] \mathrm{c} 2 \mathrm{cnncc} 2 \mathrm{c}(=\mathrm{O})[\mathrm{nH}] 1$ & 4.39 & 3.05 & 11.43 & 10.59 & 17.19 & 17.52 & 0.40 & 0.48 \\
\hline S8567 & $\mathrm{O}=\mathrm{c} 1 \mathrm{sc}(=\mathrm{O}) \mathrm{c} 2 \mathrm{c}([\mathrm{nH}] 1) \mathrm{cnnc} 2$ & 4.91 & 3.96 & 12.48 & 11.73 & 16.82 & 18.19 & 0.70 & 0.64 \\
\hline S8569 & $\mathrm{O}=\mathrm{c} 1 \mathrm{cc}[\mathrm{nH}] \mathrm{c} 2 \mathrm{c} 1 \mathrm{cnnc} 2$ & 1.14 & 0.45 & 13.77 & 11.02 & 23.52 & 23.39 & 0.34 & 0.44 \\
\hline S857 & $\mathrm{O}=\mathrm{c} 1 \mathrm{oc} 2 \mathrm{c}([\mathrm{nH}] 1) \operatorname{ccs} 2$ & -0.62 & -1.46 & 5.95 & 6.45 & 15.71 & 15.26 & 0.34 & 0.46 \\
\hline S8570 & $\mathrm{O}=\mathrm{c} 1 \mathrm{nc}[\mathrm{nH}] \mathrm{c} 2 \mathrm{c} 1 \mathrm{cnnc} 2$ & 2.96 & 2.28 & 15.90 & 13.56 & 25.57 & 26.32 & 0.38 & 0.45 \\
\hline S8571 & $\mathrm{c} 1 \mathrm{cnc} 2 \mathrm{c}(\mathrm{n} 1) \mathrm{cnnc} 2$ & 1.71 & 1.51 & 8.41 & 7.30 & 17.20 & 14.02 & 0.61 & 0.61 \\
\hline S8572 & $\mathrm{n} 1 \mathrm{cnc} 2 \mathrm{c}(\mathrm{n} 1) \mathrm{cnnc} 2$ & 3.72 & 3.34 & 8.77 & 8.49 & 13.78 & 13.73 & 0.54 & 0.68 \\
\hline S8573 & $\mathrm{O}=\mathrm{c} 1[\mathrm{nH}] \mathrm{cnc} 2 \mathrm{c} 1 \mathrm{cnnc} 2$ & 2.31 & 2.28 & 12.45 & 10.72 & 21.54 & 18.61 & 0.50 & 0.41 \\
\hline S8574 & $\mathrm{O}=\mathrm{c} 1 \mathrm{cnc} 2 \mathrm{c}(\mathrm{o} 1) \mathrm{cnnc} 2$ & 4.53 & 3.88 & 11.28 & 8.89 & 16.79 & 14.02 & 0.37 & 0.45 \\
\hline S8575 & $\mathrm{O}=\mathrm{c} 1 \mathrm{ocnc} 2 \mathrm{c} 1 \mathrm{cnnc} 2$ & 3.96 & 3.88 & 10.60 & 9.23 & 16.55 & 15.29 & 0.57 & 0.48 \\
\hline S858 & $\mathrm{O}=\mathrm{c} 1 \mathrm{oc} 2 \mathrm{c}([\mathrm{nH}] 1) \operatorname{scc} 2$ & -0.67 & -1.46 & 5.50 & 5.69 & 15.22 & 15.06 & 0.92 & 0.85 \\
\hline S8582 & $\mathrm{O}=\mathrm{c} 1[\mathrm{nH}] \mathrm{nnc} 2 \mathrm{c} 1 \mathrm{cnnc} 2$ & 4.14 & 4.11 & 10.21 & 10.71 & 15.26 & 16.04 & 0.62 & 0.55 \\
\hline S8587 & $\mathrm{O}=\mathrm{c} 1 \mathrm{c} \operatorname{coc} 2 \mathrm{c} 1 \mathrm{cnn} c 2$ & 2.36 & 2.05 & 9.53 & 8.39 & 17.33 & 17.16 & 0.31 & 0.40 \\
\hline S8588 & $\mathrm{O}=\mathrm{c} 1 \mathrm{n} \operatorname{coc} 2 \mathrm{c} 1 \mathrm{cnn} c 2$ & 4.16 & 3.88 & 12.01 & 10.04 & 17.88 & 18.85 & 0.69 & 0.63 \\
\hline S859 & $\mathrm{O}=\mathrm{c} 1 \mathrm{sc} 2 \mathrm{c}([\mathrm{nH}] 1) \operatorname{ccs} 2$ & -1.39 & -2.15 & 5.30 & 5.49 & 14.28 & 14.18 & 0.64 & 0.52 \\
\hline S8590 & $\mathrm{O}=\mathrm{c} 1 \mathrm{cn}[\mathrm{nH}] \mathrm{c} 2 \mathrm{c} 1 \mathrm{cnnc} 2$ & 2.89 & 2.28 & 11.90 & 9.99 & 18.86 & 18.56 & 0.40 & 0.49 \\
\hline S8594 & $\mathrm{O}=\mathrm{c} 1 \mathrm{nn}[\mathrm{nH}] \mathrm{c} 2 \mathrm{c} 1 \mathrm{cnnc} 2$ & 4.70 & 4.11 & 14.76 & 13.55 & 21.55 & 23.75 & 0.31 & 0.40 \\
\hline
\end{tabular}




\begin{tabular}{|c|c|c|c|c|c|c|c|c|c|}
\hline S8599 & $\mathrm{O}=\mathrm{c} 1 \mathrm{sc} 2 \mathrm{cnncc} 2 \mathrm{c}(=\mathrm{O})[\mathrm{nH}] 1$ & 4.66 & 3.96 & 10.93 & 9.83 & 15.59 & 14.55 & 0.32 & 0.38 \\
\hline $\mathbf{S 8 6 0}$ & $\mathrm{O}=\mathrm{c} 1 \mathrm{sc} 2 \mathrm{c}([\mathrm{nH}] 1) \mathrm{scc} 2$ & -1.41 & -2.15 & 4.49 & 4.78 & 13.44 & 14.00 & 0.61 & 0.51 \\
\hline S861 & $\operatorname{c} 1 \operatorname{ccc} 2 \mathrm{c}(\mathrm{c} 1) \operatorname{scc} 2$ & -4.87 & -5.28 & -0.22 & 0.71 & 8.17 & 10.30 & 0.42 & 0.51 \\
\hline S8611 & $\mathrm{O}=\mathrm{c} 1 \mathrm{cccc} 2 \mathrm{n} 1 \mathrm{cc}[\mathrm{nH}] 2$ & -3.16 & -2.84 & 9.59 & 6.49 & 24.24 & 19.41 & 0.74 & 0.83 \\
\hline S8612 & $\mathrm{O}=\mathrm{c} 1[\mathrm{nH}] \mathrm{ccc} 2 \mathrm{c} 1 \mathrm{c}[\mathrm{nH}] \mathrm{c} 2$ & -4.59 & -3.59 & 5.59 & 5.30 & 18.78 & 17.09 & 0.50 & 0.66 \\
\hline S8616 & $\mathrm{O}=\mathrm{c} 1[\mathrm{nH}] \operatorname{ccc} 2 \mathrm{c} 1 \operatorname{coc} 2$ & -2.35 & -1.99 & 4.32 & 5.21 & 14.19 & 14.69 & 0.54 & 0.48 \\
\hline S862 & $\operatorname{c} 1 \mathrm{ncc} 2 \mathrm{c}(\mathrm{c} 1) \operatorname{scc} 2$ & -2.88 & -3.45 & 2.38 & 2.18 & 10.27 & 10.89 & 0.93 & 0.97 \\
\hline S8620 & $\mathrm{O}=\mathrm{c} 1[\mathrm{nH}] \operatorname{ccc} 2 \mathrm{c} 1 \mathrm{csc} 2$ & -2.91 & -2.68 & 4.18 & 4.40 & 14.32 & 13.95 & 0.53 & 0.62 \\
\hline S8621 & $\mathrm{O}=\mathrm{c} 1 \mathrm{ccc} 2 \mathrm{c}([\mathrm{nH}] 1) \csc 2$ & -1.63 & -2.68 & 3.70 & 4.05 & 14.24 & 13.81 & 0.49 & 0.60 \\
\hline S8623 & $\mathrm{O}=\mathrm{c} 1 \mathrm{cccc} 2 \mathrm{n} 1 \mathrm{cn}[\mathrm{nH}] 2$ & -1.01 & -1.01 & 8.36 & 7.88 & 19.49 & 19.61 & 0.67 & 0.66 \\
\hline S8624 & $\mathrm{O}=\mathrm{c} 1[\mathrm{nH}] \operatorname{ccc} 2 \mathrm{c} 1 \mathrm{c}[\mathrm{nH}] \mathrm{n} 2$ & -2.81 & -1.76 & 5.09 & 5.95 & 15.04 & 15.20 & 0.57 & 0.66 \\
\hline S8625 & $\mathrm{O}=\mathrm{c} 1[\mathrm{nH}] \mathrm{ccc} 2 \mathrm{c} 1 \mathrm{n}[\mathrm{nH}] \mathrm{c} 2$ & -2.96 & -1.76 & 5.70 & 7.22 & 20.20 & 19.68 & 0.32 & 0.44 \\
\hline S8626 & $\mathrm{O}=\mathrm{c} 1 \mathrm{ccc} 2 \mathrm{c}([\mathrm{nH}] 1) \mathrm{n}[\mathrm{nH}] \mathrm{c} 2$ & -1.83 & -1.76 & 7.84 & 6.52 & 20.11 & 17.30 & 0.75 & 0.65 \\
\hline S863 & $\operatorname{c} 1 \mathrm{ncc} 2 \mathrm{c}(\mathrm{c} 1) \operatorname{ccs} 2$ & -2.90 & -3.45 & 2.72 & 2.38 & 10.81 & 10.97 & 0.42 & 0.50 \\
\hline S8630 & $\mathrm{O}=\mathrm{c} 1[\mathrm{nH}] \operatorname{ccc} 2 \mathrm{c} 1 \operatorname{con} 2$ & -0.23 & -0.16 & 7.22 & 6.82 & 13.63 & 14.14 & 0.31 & 0.42 \\
\hline S8631 & $\mathrm{O}=\mathrm{c} 1[\mathrm{nH}] \mathrm{ccc} 2 \mathrm{c} 1 \mathrm{noc} 2$ & -0.39 & -0.16 & 7.18 & 8.08 & 18.75 & 18.62 & 0.53 & 0.52 \\
\hline S8635 & $\mathrm{O}=\mathrm{c} 1 \operatorname{ccc} 2 \mathrm{n} 1 \mathrm{cns} 2$ & 0.15 & -0.10 & 4.61 & 6.51 & 12.69 & 15.43 & 0.97 & 0.97 \\
\hline S864 & $\operatorname{c} 1 \mathrm{cnc} 2 \mathrm{c}(\mathrm{c} 1) \operatorname{ccs} 2$ & -3.40 & -3.45 & 2.29 & 2.65 & 10.66 & 11.08 & 0.52 & 0.38 \\
\hline S8640 & $\mathrm{O}=\mathrm{c} 1 \mathrm{cccc} 2 \mathrm{n} 1[\mathrm{nH}] \mathrm{cn} 2$ & -1.24 & -1.01 & 8.58 & 7.50 & 17.94 & 16.50 & 0.37 & 0.46 \\
\hline S8645 & $\mathrm{O}=\mathrm{c} 1 \mathrm{ccc} 2 \mathrm{n} 1 \mathrm{ncs} 2$ & -0.94 & -0.10 & 6.26 & 6.45 & 19.60 & 17.51 & 0.73 & 0.68 \\
\hline S8648 & $\mathrm{O}=\mathrm{c} 1[\mathrm{nH}] \operatorname{ccc} 2 \mathrm{c} 1 \mathrm{n}[\mathrm{nH}] \mathrm{n} 2$ & -1.10 & 0.07 & 5.88 & 6.85 & 16.87 & 15.54 & 0.39 & 0.43 \\
\hline S8649 & $\mathrm{O}=\mathrm{c} 1 \mathrm{ccc} 2 \mathrm{c}([\mathrm{nH}] 1) \mathrm{n}[\mathrm{nH}] \mathrm{n} 2$ & -0.13 & 0.07 & 6.27 & 6.69 & 15.43 & 14.35 & 0.41 & 0.49 \\
\hline S865 & $\mathrm{c} 1 \mathrm{cnc} 2 \mathrm{c}(\mathrm{c} 1) \operatorname{scc} 2$ & -3.48 & -3.45 & 1.60 & 2.11 & 9.47 & 10.86 & 0.75 & 0.67 \\
\hline S8655 & $\mathrm{O}=\mathrm{c} 1[\mathrm{nH}] \mathrm{ccc} 2 \mathrm{c} 1$ non 2 & 1.80 & 1.67 & 10.45 & 8.66 & 17.86 & 15.82 & 0.41 & 0.53 \\
\hline S8656 & $\mathrm{O}=\mathrm{c} 1 \mathrm{ccc} 2 \mathrm{c}([\mathrm{nH}] 1)$ non 2 & 2.73 & 1.67 & 6.77 & 6.71 & 10.95 & 12.14 & 0.69 & 0.69 \\
\hline S8657 & $\mathrm{O}=\mathrm{c} 1[\mathrm{nH}] \operatorname{ccc} 2 \mathrm{c} 1 \mathrm{nsn} 2$ & 0.38 & 0.98 & 7.89 & 7.73 & 17.40 & 14.75 & 0.73 & 0.65 \\
\hline S8658 & $\mathrm{O}=\mathrm{c} 1 \mathrm{ccc} 2 \mathrm{c}([\mathrm{nH}] 1) \mathrm{nsn} 2$ & 1.48 & 0.98 & 4.52 & 5.90 & 11.24 & 11.38 & 0.86 & 0.73 \\
\hline S866 & $\mathrm{c} 1 \mathrm{nnnc} 2 \mathrm{c}(\mathrm{c} 1) \operatorname{scc} 2$ & -1.41 & -1.62 & 4.06 & 4.60 & 14.19 & 13.69 & 0.76 & 0.63 \\
\hline S8664 & $\mathrm{O}=\mathrm{c} 1 \mathrm{ccc} 2 \mathrm{n} 1 \mathrm{ccc} 2$ & -2.30 & -2.46 & 4.44 & 4.36 & 16.04 & 15.08 & 0.75 & 0.74 \\
\hline S8665 & $\mathrm{O}=\mathrm{c} 1 \mathrm{ccc} 2 \mathrm{n} 1 \mathrm{ccnc} 2$ & -0.31 & -0.63 & 4.52 & 6.08 & 13.37 & 15.33 & 0.42 & 0.54 \\
\hline S8666 & $\mathrm{O}=\mathrm{c} 1 \mathrm{ccc} 2 \mathrm{n} 1 \mathrm{cnc} 2$ & -0.37 & -0.63 & 5.40 & 6.67 & 14.62 & 17.27 & 0.61 & 0.49 \\
\hline
\end{tabular}




\begin{tabular}{|c|c|c|c|c|c|c|c|c|c|}
\hline S8667 & $\mathrm{O}=\mathrm{c} 1 \mathrm{cccc} 2 \mathrm{n} 1 \mathrm{nccc} 2$ & -1.40 & -0.63 & 7.03 & 6.77 & 21.71 & 18.63 & 0.29 & 0.30 \\
\hline S8668 & $\mathrm{O}=\mathrm{c} 1 \mathrm{ccc} 2 \mathrm{n} 1 \mathrm{ccc} 2$ & -1.06 & -0.63 & 5.32 & 5.42 & 14.49 & 13.75 & 0.66 & 0.66 \\
\hline S867 & $\mathrm{c} 1 \mathrm{nnc} 2 \mathrm{c}(\mathrm{c} 1) \mathrm{ccs} 2$ & -1.31 & -1.62 & 5.02 & 5.33 & 15.40 & 14.00 & 0.33 & 0.42 \\
\hline S8672 & $\mathrm{O}=\mathrm{c} 1[\mathrm{nH}] \operatorname{ccc} 2 \mathrm{c} 1 \mathrm{c}(=\mathrm{O})[\mathrm{nH}] \mathrm{cc} 2$ & -2.17 & -0.61 & 6.27 & 7.84 & 23.51 & 21.12 & 0.49 & 0.62 \\
\hline S8673 & $\mathrm{O}=\mathrm{c} 1[\mathrm{nH}] \operatorname{ccc} 2 \mathrm{c} 1 \mathrm{cc}[\mathrm{nH}] \mathrm{c} 2=\mathrm{O}$ & -1.43 & -0.61 & 5.11 & 6.96 & 13.14 & 16.00 & 0.59 & 0.68 \\
\hline S8676 & $\mathrm{O}=\mathrm{c} 1 \mathrm{ccc} 2 \mathrm{c}([\mathrm{nH}] 1)[\mathrm{nH}] \mathrm{c}(=\mathrm{O}) \mathrm{cc} 2$ & 1.66 & -0.61 & 8.31 & 7.90 & 15.33 & 16.87 & 0.45 & 0.54 \\
\hline S8677 & $\mathrm{O}=\mathrm{c} 1 \mathrm{ccc} 2 \mathrm{c}([\mathrm{nH}] 1) \operatorname{ccc}(=\mathrm{O})[\mathrm{nH}] 2$ & 1.80 & -0.61 & 7.36 & 6.95 & 15.17 & 16.58 & 0.52 & 0.62 \\
\hline S8678 & $\mathrm{O}=\mathrm{c} 1 \mathrm{cccc} 2 \mathrm{n} 1 \mathrm{cc}[\mathrm{nH}] \mathrm{c} 2=\mathrm{O}$ & -0.50 & 0.14 & 7.25 & 7.14 & 15.95 & 16.23 & 0.67 & 0.53 \\
\hline S8679 & $\mathrm{O}=\mathrm{c} 1 \mathrm{ccc} 2 \mathrm{n} 1 \mathrm{c}(=\mathrm{O})[\mathrm{nH}] \mathrm{cc} 2$ & -1.31 & 0.14 & 8.44 & 7.97 & 23.55 & 21.53 & 0.63 & 0.52 \\
\hline S868 & $\mathrm{n} 1 \mathrm{ncc} 2 \mathrm{c}(\mathrm{c} 1) \operatorname{scc} 2$ & -0.71 & -1.62 & 5.69 & 4.87 & 16.58 & 13.80 & 0.55 & 0.45 \\
\hline S8681 & $\mathrm{O}=\mathrm{c} 1 \mathrm{ccn} 2 \mathrm{c}([\mathrm{nH}] 1) \operatorname{ccc} 2=\mathrm{O}$ & 1.02 & 0.14 & 6.73 & 8.62 & 13.81 & 19.67 & 0.65 & 0.66 \\
\hline S8682 & $\mathrm{O}=\mathrm{c} 1[\mathrm{nH}] \mathrm{cc} 2 \mathrm{c}(\mathrm{c} 1) \mathrm{cc}[\mathrm{nH}] \mathrm{c} 2=\mathrm{O}$ & -0.36 & -0.61 & 7.29 & 7.21 & 17.64 & 17.01 & 0.42 & 0.51 \\
\hline S8683 & $\mathrm{O}=\mathrm{c} 1[\mathrm{nH}] \mathrm{cc} 2 \mathrm{c}(\mathrm{c} 1) \mathrm{c}(=\mathrm{O})[\mathrm{nH}] \mathrm{cc} 2$ & -0.50 & -0.61 & 8.02 & 8.29 & 19.70 & 20.83 & 0.32 & 0.33 \\
\hline S8686 & $\mathrm{O}=\mathrm{c} 1 \mathrm{occc} 2 \mathrm{c} 1 \mathrm{c}(=\mathrm{O})[\mathrm{nH}] \mathrm{cc} 2$ & -0.38 & 0.99 & 9.08 & 9.66 & 24.62 & 22.48 & 0.66 & 0.67 \\
\hline S8687 & $\mathrm{O}=\mathrm{c} 1 \mathrm{occc} 2 \mathrm{c} 1 \mathrm{cc}[\mathrm{nH}] \mathrm{c} 2=\mathrm{O}$ & 0.34 & 0.99 & 7.87 & 8.05 & 15.57 & 15.68 & 0.63 & 0.55 \\
\hline S869 & $\mathrm{O}=\mathrm{c} 1[\mathrm{nH}] \operatorname{ccc} 2 \mathrm{c} 1 \mathrm{ccs} 2$ & -2.85 & -2.68 & 5.26 & 4.15 & 16.03 & 13.82 & 0.41 & 0.52 \\
\hline S8690 & $\mathrm{O}=\mathrm{c} 1 \mathrm{ccc} 2 \mathrm{c}(\mathrm{o} 1) \mathrm{cc}[\mathrm{nH}] \mathrm{c} 2=\mathrm{O}$ & 0.85 & 0.99 & 10.00 & 8.56 & 19.13 & 17.77 & 0.32 & 0.41 \\
\hline S87 & $\mathrm{O}=\mathrm{c} 1 \mathrm{ncncs} 1$ & 2.38 & 2.32 & 7.96 & 6.38 & 15.52 & 13.41 & 0.62 & 0.77 \\
\hline S870 & $\mathrm{O}=\mathrm{c} 1[\mathrm{nH}] \mathrm{ccc} 2 \mathrm{c} 1 \mathrm{scc} 2$ & -2.86 & -2.68 & 4.93 & 4.52 & 16.72 & 14.07 & 0.59 & 0.49 \\
\hline S871 & $\mathrm{O}=\mathrm{c} 1 \mathrm{ccc} 2 \mathrm{c}([\mathrm{nH}] 1) \operatorname{scc} 2$ & -1.52 & -2.68 & 3.45 & 3.92 & 14.44 & 13.82 & 0.47 & 0.54 \\
\hline S8718 & $\mathrm{O}=\mathrm{c} 1[\mathrm{nH}] \mathrm{ccc} 2 \mathrm{c} 1 \mathrm{ncnc} 2$ & 0.03 & 0.45 & 8.19 & 7.99 & 19.36 & 17.64 & 0.30 & 0.40 \\
\hline S8719 & $\mathrm{O}=\mathrm{c} 1[\mathrm{nH}] \mathrm{ccc} 2 \mathrm{c} 1 \mathrm{cncn} 2$ & 0.08 & 0.45 & 8.46 & 7.18 & 14.94 & 14.75 & 0.31 & 0.41 \\
\hline S872 & $\mathrm{O}=\mathrm{c} 1 \mathrm{ccc} 2 \mathrm{c}([\mathrm{nH}] 1) \operatorname{ccs} 2$ & -1.53 & -2.68 & 4.46 & 4.31 & 15.94 & 13.89 & 0.32 & 0.39 \\
\hline S8720 & $\mathrm{O}=\mathrm{c} 1 \mathrm{ccc} 2 \mathrm{c}([\mathrm{nH}] 1) \operatorname{cncn} 2$ & 1.50 & 0.45 & 6.72 & 6.63 & 13.40 & 13.54 & 0.38 & 0.49 \\
\hline S8721 & $\mathrm{O}=\mathrm{c} 1 \mathrm{ccc} 2 \mathrm{c}([\mathrm{nH}] 1) \mathrm{ncnc} 2$ & 1.41 & 0.45 & 6.55 & 5.79 & 13.99 & 12.86 & 0.65 & 0.62 \\
\hline S8722 & $\mathrm{O}=\mathrm{c} 1 \mathrm{cccc} 2 \mathrm{n} 1 \mathrm{cncn} 2$ & 0.96 & 1.20 & 4.94 & 7.14 & 11.63 & 14.65 & 0.63 & 0.55 \\
\hline S8736 & $\mathrm{O}=\mathrm{c} 1[\mathrm{nH}] \mathrm{nc} 2 \mathrm{c}(\mathrm{c} 1) \mathrm{cc}[\mathrm{nH}] \mathrm{c} 2=\mathrm{O}$ & 0.94 & 1.22 & 9.30 & 7.87 & 18.09 & 16.94 & 0.39 & 0.43 \\
\hline S8737 & $\mathrm{O}=\mathrm{c} 1[\mathrm{nH}] \mathrm{nc} 2 \mathrm{c}(\mathrm{c} 1) \mathrm{c}(=\mathrm{O})[\mathrm{nH}] \mathrm{cc} 2$ & 0.93 & 1.22 & 8.73 & 7.66 & 18.39 & 16.13 & 0.98 & 1.00 \\
\hline S8742 & $\mathrm{O}=\mathrm{c} 1 \mathrm{ccc} 2 \mathrm{c}([\mathrm{nH}] 1) \operatorname{cnc}(=\mathrm{O})[\mathrm{nH}] 2$ & 3.68 & 1.22 & 10.51 & 9.61 & 20.43 & 19.84 & 0.44 & 0.57 \\
\hline S8743 & $\mathrm{O}=\mathrm{c} 1 \mathrm{ccc} 2 \mathrm{c}([\mathrm{nH}] 1)[\mathrm{nH}] \mathrm{c}(=\mathrm{O}) \mathrm{nc} 2$ & 3.53 & 1.22 & 11.39 & 11.06 & 20.71 & 20.53 & 0.77 & 0.65 \\
\hline S875 & $\mathrm{O}=\mathrm{c} 1 \mathrm{occc} 2 \mathrm{c} 1 \mathrm{ccs} 2$ & -1.19 & -1.08 & 4.30 & 4.62 & 14.49 & 13.50 & 0.57 & 0.51 \\
\hline
\end{tabular}




\begin{tabular}{|c|c|c|c|c|c|c|c|c|c|}
\hline S876 & $\mathrm{O}=\mathrm{c} 1 \mathrm{occc} 2 \mathrm{c} 1 \mathrm{scc} 2$ & -1.18 & -1.08 & 4.72 & 5.28 & 15.65 & 13.83 & 0.33 & 0.41 \\
\hline S877 & $\mathrm{O}=\mathrm{c} 1 \mathrm{ccc} 2 \mathrm{c}(\mathrm{o} 1) \operatorname{scc} 2$ & -0.57 & -1.08 & 5.23 & 5.09 & 15.43 & 13.69 & 0.65 & 0.60 \\
\hline S878 & $\mathrm{O}=\mathrm{c} 1 \mathrm{ccc} 2 \mathrm{c}(\mathrm{o} 1) \operatorname{ccs} 2$ & -0.61 & -1.08 & 4.50 & 4.67 & 15.14 & 13.53 & 0.43 & 0.54 \\
\hline S8790 & $\mathrm{O}=\mathrm{c} 1 \mathrm{cnc} 2 \mathrm{c}([\mathrm{nH}] 1) \mathrm{cc}[\mathrm{nH}] \mathrm{c} 2=\mathrm{O}$ & 1.22 & 1.22 & 10.52 & 11.51 & 21.68 & 23.20 & 0.61 & 0.53 \\
\hline S8791 & $\mathrm{O}=\mathrm{c} 1 \mathrm{cnc} 2 \mathrm{c}([\mathrm{nH}] 1) \mathrm{c}(=\mathrm{O})[\mathrm{nH}] \mathrm{cc} 2$ & 1.33 & 1.22 & 9.05 & 9.30 & 17.79 & 17.36 & 0.69 & 0.58 \\
\hline S8792 & $\mathrm{O}=\mathrm{c} 1 \mathrm{ccc} 2 \mathrm{c}([\mathrm{nH}] 1)[\mathrm{nH}] \mathrm{c}(=\mathrm{O}) \mathrm{cn} 2$ & 2.84 & 1.22 & 11.40 & 10.50 & 17.37 & 17.75 & 0.37 & 0.47 \\
\hline S8793 & $\mathrm{O}=\mathrm{c} 1 \mathrm{cnc} 2 \mathrm{c}([\mathrm{nH}] 1) \operatorname{ccc}(=\mathrm{O})[\mathrm{nH}] 2$ & 2.91 & 1.22 & 10.08 & 8.20 & 18.05 & 16.37 & 0.66 & 0.62 \\
\hline S8798 & $\mathrm{O}=\mathrm{c} 1 \mathrm{ccc} 2 \mathrm{c}([\mathrm{nH}] 1)[\mathrm{nH}] \mathrm{c}(=\mathrm{O}) \mathrm{nn} 2$ & 4.76 & 3.05 & 14.94 & 14.68 & 22.53 & 23.66 & 0.77 & 0.69 \\
\hline S88 & c1cnnnn 1 & 3.09 & 2.47 & 10.60 & 8.07 & 16.78 & 15.72 & 0.57 & 0.47 \\
\hline S8802 & $\mathrm{O}=\mathrm{c} 1[\mathrm{nH}] \mathrm{c} 2 \mathrm{cc}[\mathrm{nH}] \mathrm{c}(=\mathrm{O}) \mathrm{c} 2 \mathrm{c}(=\mathrm{O})[\mathrm{nH}] 1$ & 1.35 & 1.99 & 11.48 & 10.70 & 24.94 & 22.67 & 0.87 & 0.76 \\
\hline S8803 & $\mathrm{O}=\mathrm{c} 1[\mathrm{nH}] \mathrm{c}(=\mathrm{O}) \mathrm{c} 2 \mathrm{c}([\mathrm{nH}] 1) \mathrm{c}(=\mathrm{O})[\mathrm{nH}] \mathrm{cc} 2$ & 2.05 & 1.99 & 9.60 & 8.91 & 16.53 & 16.35 & 0.40 & 0.52 \\
\hline S8804 & $\mathrm{O}=\mathrm{c} 1 \mathrm{ccc} 2 \mathrm{c}([\mathrm{nH}] 1)[\mathrm{nH}] \mathrm{c}(=\mathrm{O})[\mathrm{nH}] \mathrm{c} 2=\mathrm{O}$ & 3.48 & 1.99 & 12.65 & 10.77 & 18.07 & 18.42 & 0.30 & 0.37 \\
\hline S8805 & $\mathrm{O}=\mathrm{c} 1 \mathrm{ccc} 2 \mathrm{c}([\mathrm{nH}] 1) \mathrm{c}(=\mathrm{O})[\mathrm{nH}] \mathrm{c}(=\mathrm{O})[\mathrm{nH}] 2$ & 3.55 & 1.99 & 11.25 & 8.90 & 19.35 & 16.93 & 0.35 & 0.45 \\
\hline S8807 & $\mathrm{O}=\mathrm{c} 1[\mathrm{nH}] \mathrm{c} 2 \operatorname{cccc}(=\mathrm{O}) \mathrm{n} 2 \mathrm{c}(=\mathrm{O})[\mathrm{nH}] 1$ & 2.27 & 2.74 & 9.67 & 10.77 & 20.60 & 22.77 & 0.57 & 51 \\
\hline S8809 & $\mathrm{O}=\mathrm{c} 1 \mathrm{ccc} 2 \mathrm{c}([\mathrm{nH}] 1)[\mathrm{nH}] \mathrm{c}(=\mathrm{O}) \mathrm{c}(=\mathrm{O})[\mathrm{nH}] 2$ & 4.07 & 1.99 & 11.62 & 12.01 & 19.65 & 24.51 & 0.77 & 0.66 \\
\hline S8825 & $\mathrm{O}=\mathrm{c} 1 \mathrm{ccc} 2 \mathrm{c}([\mathrm{nH}] 1)[\mathrm{nH}] \mathrm{c}(=\mathrm{O}) \mathrm{sc} 2=\mathrm{O}$ & 4.07 & 2.90 & 13.65 & 12.78 & 17.70 & 19.70 & 0.38 & 0.39 \\
\hline S8826 & $\mathrm{O}=\mathrm{c} 1 \mathrm{ccc} 2 \mathrm{c}([\mathrm{nH}] 1) \mathrm{c}(=\mathrm{O}) \mathrm{sc}(=\mathrm{O})[\mathrm{nH}] 2$ & 4.12 & 2.90 & 12.43 & 10.20 & 19.49 & 17.86 & 0.64 & 0.55 \\
\hline S8839 & $\mathrm{O}=\mathrm{c} 1 \operatorname{ccc} 2 \mathrm{n} 1 \mathrm{c}(=\mathrm{O}) \mathrm{cc}[\mathrm{nH}] 2$ & -1.14 & 0.14 & 12.42 & 11.88 & 29.03 & 29.26 & 0.36 & 0.46 \\
\hline S884 & $\mathrm{O}=\mathrm{c} 1 \mathrm{ccc} 2 \mathrm{c}(\mathrm{s} 1) \operatorname{ccs} 2$ & -1.21 & -1.77 & 4.67 & 3.83 & 14.64 & 12.54 & 0.73 & 0.62 \\
\hline S8841 & $\mathrm{O}=\mathrm{c} 1 \mathrm{nc}[\mathrm{nH}] \mathrm{c} 2 \mathrm{c} 1 \mathrm{cc}[\mathrm{nH}] \mathrm{c} 2=\mathrm{O}$ & 0.98 & 1.22 & 8.98 & 12.50 & 21.63 & 23.41 & 0.33 & 0.42 \\
\hline S8842 & $\mathrm{O}=\mathrm{c} 1 \mathrm{nc}[\mathrm{nH}] \mathrm{c} 2 \mathrm{c} 1 \mathrm{c}(=\mathrm{O})[\mathrm{nH}] \mathrm{cc} 2$ & -0.05 & 1.22 & 13.30 & 15.85 & 32.51 & 35.30 & 0.71 & 0.64 \\
\hline S8843 & $\mathrm{O}=\mathrm{c} 1 \mathrm{ccc} 2 \mathrm{c}([\mathrm{nH}] 1) \mathrm{c}(=\mathrm{O}) \mathrm{nc}[\mathrm{nH}] 2$ & 2.09 & 1.22 & 15.42 & 11.43 & 26.45 & 25.27 & 0.57 & 0.45 \\
\hline S8844 & $\mathrm{O}=\mathrm{c} 1 \mathrm{ccc} 2 \mathrm{c}([\mathrm{nH}] 1)[\mathrm{nH}] \mathrm{cnc} 2=\mathrm{O}$ & 2.15 & 1.22 & 16.57 & 14.93 & 28.11 & 28.08 & 0.67 & 0.57 \\
\hline S8847 & $\mathrm{O}=\mathrm{c} 1[\mathrm{nH}] \operatorname{ccc} 2 \mathrm{c} 1 \mathrm{nccn} 2$ & -0.76 & 0.45 & 6.61 & 6.84 & 17.86 & 14.99 & 0.30 & 0.40 \\
\hline S8848 & $\mathrm{O}=\mathrm{c} 1 \mathrm{ccc} 2 \mathrm{c}([\mathrm{nH}] 1) \mathrm{nccn} 2$ & 0.68 & 0.45 & 4.64 & 6.08 & 13.12 & 13.34 & 0.40 & 0.53 \\
\hline $\mathbf{S 8 8 5 0}$ & $\mathrm{O}=\mathrm{c} 1[\mathrm{nH}] \mathrm{ccc} 2 \mathrm{c} 1 \mathrm{ncnn} 2$ & 1.48 & 2.28 & 9.64 & 9.15 & 17.38 & 16.53 & 0.51 & 0.42 \\
\hline S8851 & $\mathrm{O}=\mathrm{c} 1[\mathrm{nH}] \mathrm{ccc} 2 \mathrm{c} 1 \mathrm{nncn} 2$ & 1.44 & 2.28 & 10.32 & 9.64 & 20.24 & 18.27 & 0.64 & 0.52 \\
\hline S8852 & $\mathrm{O}=\mathrm{c} 1 \mathrm{ccc} 2 \mathrm{c}([\mathrm{nH}] 1) \mathrm{nnncn} 2$ & 2.84 & 2.28 & 7.24 & 7.38 & 12.64 & 13.37 & 0.31 & 0.33 \\
\hline S8853 & $\mathrm{O}=\mathrm{c} 1 \mathrm{ccc} 2 \mathrm{c}([\mathrm{nH}] 1) \mathrm{ncnn} 2$ & 2.83 & 2.28 & 7.33 & 7.89 & 12.07 & 13.79 & 0.68 & 0.65 \\
\hline S8856 & $\mathrm{O}=\mathrm{c} 1[\mathrm{nH}] \mathrm{ccc} 2 \mathrm{c} 1 \mathrm{nc}[\mathrm{nH}] \mathrm{c} 2=\mathrm{O}$ & -0.19 & 1.22 & 7.32 & 8.95 & 18.55 & 17.40 & 0.37 & 0.47 \\
\hline S8857 & $\mathrm{O}=\mathrm{c} 1[\mathrm{nH}] \operatorname{ccc} 2 \mathrm{c} 1 \mathrm{c}(=\mathrm{O})[\mathrm{nH}] \mathrm{cn} 2$ & -0.89 & 1.22 & 7.27 & 8.53 & 21.89 & 17.88 & 0.27 & 0.35 \\
\hline
\end{tabular}




\begin{tabular}{|c|c|c|c|c|c|c|c|c|c|}
\hline S8858 & $\mathrm{O}=\mathrm{c} 1 \mathrm{ccc} 2 \mathrm{c}([\mathrm{nH}] 1) \mathrm{c}(=\mathrm{O})[\mathrm{nH}] \mathrm{cn} 2$ & 1.27 & 1.22 & 10.53 & 9.29 & 20.92 & 17.32 & 0.63 & 0.63 \\
\hline S8859 & $\mathrm{O}=\mathrm{c} 1 \mathrm{ccc} 2 \mathrm{c}([\mathrm{nH}] 1) \mathrm{nc}[\mathrm{nH}] \mathrm{c} 2=\mathrm{O}$ & 1.14 & 1.22 & 11.26 & 8.85 & 20.88 & 17.43 & 0.62 & 0.50 \\
\hline S8862 & $\mathrm{O}=\mathrm{c} 1 \mathrm{cnc} 2 \mathrm{c}(\mathrm{o} 1) \mathrm{cc}[\mathrm{nH}] \mathrm{c} 2=\mathrm{O}$ & 2.04 & 2.82 & 10.77 & 9.97 & 19.83 & 19.08 & 0.71 & 0.57 \\
\hline S887 & $\mathrm{c} 1 \mathrm{ncc} 2 \mathrm{c}(\mathrm{n} 1) \operatorname{ccs} 2$ & -1.47 & -1.62 & 3.79 & 3.18 & 9.13 & 10.23 & 0.60 & 0.48 \\
\hline S888 & $\mathrm{c} 1 \mathrm{ncc} 2 \mathrm{c}(\mathrm{n} 1) \operatorname{scc} 2$ & -1.38 & -1.62 & 3.80 & 3.53 & 9.37 & 10.37 & 0.43 & 0.50 \\
\hline S8882 & $\mathrm{O}=\mathrm{c} 1 \mathrm{ccc} 2 \mathrm{c}([\mathrm{nH}] 1) \mathrm{c}(=\mathrm{O}) \operatorname{sen} 2$ & 2.13 & 2.13 & 7.24 & 7.23 & 16.09 & 13.64 & 0.69 & 0.56 \\
\hline $\mathbf{S 8 8 8 3}$ & $\mathrm{O}=\mathrm{c} 1 \mathrm{ccc} 2 \mathrm{c}([\mathrm{nH}] 1) \mathrm{ncsc} 2=\mathrm{O}$ & 2.06 & 2.13 & 8.13 & 7.51 & 15.90 & 14.10 & 0.51 & 0.59 \\
\hline S8887 & $\mathrm{O}=\mathrm{c} 1 \mathrm{ccc} 2 \mathrm{c}([\mathrm{nH}] 1) \mathrm{nnnn} 2$ & 5.09 & 4.11 & 11.43 & 10.21 & 16.68 & 16.07 & 0.55 & 0.47 \\
\hline S889 & $\mathrm{c} 1 \mathrm{cc} 2 \mathrm{c}(\mathrm{s} 1) \mathrm{cnnn} 2$ & 0.76 & 0.21 & 7.07 & 6.70 & 17.45 & 15.31 & 0.50 & 0.64 \\
\hline $\mathbf{S 8 9 0}$ & $\mathrm{c} 1 \mathrm{cc} 2 \mathrm{c}(\mathrm{s} 1) \mathrm{nnn} \mathrm{c} 2$ & 0.86 & 0.21 & 7.38 & 7.24 & 17.76 & 15.54 & 0.71 & 0.60 \\
\hline S891 & $\mathrm{O}=\mathrm{c} 1[\mathrm{nH}] \mathrm{ncc} 2 \mathrm{c} 1 \mathrm{ccs} 2$ & -0.93 & -0.85 & 3.82 & 4.02 & 12.93 & 10.90 & 0.52 & 0.66 \\
\hline S892 & $\mathrm{O}=\mathrm{c} 1[\mathrm{nH}] \mathrm{ncc} 2 \mathrm{c} 1 \mathrm{scc} 2$ & -0.89 & -0.85 & 4.90 & 4.20 & 14.71 & 11.07 & 0.67 & 0.52 \\
\hline S8932 & $\mathrm{O}=\mathrm{c} 1 \mathrm{c} \operatorname{coc} 2 \mathrm{c} 1 \mathrm{c}(=\mathrm{O})[\mathrm{nH}] \mathrm{cc} 2$ & -0.77 & 0.99 & 8.73 & 8.31 & 24.88 & 20.78 & 0.60 & 0.57 \\
\hline S895 & $\mathrm{O}=\mathrm{c} 1 \mathrm{ncc} 2 \mathrm{c}([\mathrm{nH}] 1) \operatorname{ccs} 2$ & 0.38 & -0.85 & 7.32 & 7.76 & 20.90 & 18.73 & 0.67 & 0.67 \\
\hline S896 & $\mathrm{O}=\mathrm{c} 1 \mathrm{ncc} 2 \mathrm{c}([\mathrm{nH}] 1) \operatorname{scc} 2$ & 0.39 & -0.85 & 6.07 & 7.17 & 18.97 & 18.58 & 0.71 & 0.60 \\
\hline S9 & c1nces1 & -2.53 & -2.49 & 1.87 & 2.03 & 8.82 & 10.82 & 0.30 & 0.40 \\
\hline S9057 & $\mathrm{O}=\mathrm{c} 1 \mathrm{ccc} 2 \mathrm{c}(\mathrm{o} 1) \operatorname{coc} 2$ & -0.17 & -0.39 & 5.91 & 5.44 & 15.68 & 13.98 & 0.35 & 0.46 \\
\hline S9059 & $\mathrm{O}=\mathrm{c} 1 \mathrm{ccc} 2 \mathrm{c}(\mathrm{o} 1) \csc 2$ & -0.64 & -1.08 & 5.53 & 4.75 & 15.69 & 13.55 & 0.70 & 0.61 \\
\hline S9067 & $\mathrm{O}=\mathrm{c} 1 \mathrm{ccc} 2 \mathrm{c}(\mathrm{o} 1) \operatorname{con} 2$ & 1.82 & 1.44 & 5.38 & 6.45 & 11.63 & 13.01 & 0.59 & 0.63 \\
\hline S9072 & $\mathrm{O}=\mathrm{c} 1 \mathrm{occc} 2 \mathrm{c} 1 \mathrm{n}[\mathrm{nH}] \mathrm{n} 2$ & 0.52 & 1.67 & 6.58 & 7.92 & 16.27 & 15.43 & 0.59 & 0.49 \\
\hline S9080 & $\mathrm{O}=\mathrm{c} 1[\mathrm{nH}] \mathrm{cc} 2 \mathrm{c}(\mathrm{c} 1) \mathrm{oc}(=\mathrm{O}) \mathrm{cc} 2$ & 1.86 & 0.99 & 12.10 & 9.10 & 20.53 & 18.57 & 0.48 & 0.57 \\
\hline S9081 & $\mathrm{O}=\mathrm{c} 1[\mathrm{nH}] \mathrm{cc} 2 \mathrm{c}(\mathrm{c} 1) \mathrm{ccc}(=\mathrm{O}) \mathrm{o} 2$ & 2.03 & 0.99 & 7.29 & 7.82 & 15.04 & 16.14 & 0.72 & 0.64 \\
\hline S9082 & $\mathrm{O}=\mathrm{c} 1 \mathrm{occc} 2 \mathrm{c} 1 \mathrm{c}(=\mathrm{O}) \mathrm{occ} 2$ & 1.37 & 2.59 & 9.27 & 10.82 & 22.44 & 23.24 & 0.62 & 0.66 \\
\hline S9083 & $\mathrm{O}=\mathrm{c} 1 \mathrm{occc} 2 \mathrm{c} 1 \mathrm{ccoc} 2=\mathrm{O}$ & 2.09 & 2.59 & 5.20 & 7.86 & 10.54 & 14.21 & 0.36 & 0.46 \\
\hline S91 & $\mathrm{O}=\mathrm{c} 1 \mathrm{ncc} \cos 1$ & 2.46 & 2.32 & 9.53 & 6.90 & 17.58 & 14.00 & 0.33 & 0.44 \\
\hline S9104 & $\mathrm{O}=\mathrm{c} 1 \mathrm{occc} 2 \mathrm{c} 1 \mathrm{ncnc} 2$ & 1.72 & 2.05 & 7.85 & 8.41 & 17.36 & 16.99 & 0.30 & 0.41 \\
\hline S9105 & $\mathrm{O}=\mathrm{c} 1 \mathrm{occc} 2 \mathrm{c} 1 \mathrm{cncn} 2$ & 1.77 & 2.05 & 6.04 & 6.93 & 11.97 & 13.15 & 0.76 & 0.65 \\
\hline S9106 & $\mathrm{O}=\mathrm{c} 1 \mathrm{ccc} 2 \mathrm{c}(\mathrm{o} 1) \mathrm{cncn} 2$ & 2.37 & 2.05 & 6.17 & 6.75 & 12.37 & 12.47 & 0.86 & 0.82 \\
\hline S9110 & $\mathrm{O}=\mathrm{c} 1 \mathrm{ccc} 2 \mathrm{c}(\mathrm{o} 1) \mathrm{cnnn} 2$ & 4.59 & 3.88 & 10.49 & 9.66 & 16.25 & 15.75 & 0.61 & 0.50 \\
\hline S9127 & $\mathrm{O}=\mathrm{c} 1[\mathrm{nH}] \mathrm{cc} 2 \mathrm{c}(\mathrm{n} 1) \operatorname{oc}(=\mathrm{O}) \mathrm{cc} 2$ & 3.26 & 2.82 & 15.16 & 13.38 & 25.71 & 25.00 & 0.55 & 0.47 \\
\hline S915 & $\mathrm{O}=\mathrm{c} 1 \mathrm{cnc} 2 \mathrm{c}([\mathrm{nH}] 1) \operatorname{scc} 2$ & -0.20 & -0.85 & 5.16 & 5.81 & 13.28 & 14.33 & 0.51 & 0.41 \\
\hline
\end{tabular}




\begin{tabular}{|c|c|c|c|c|c|c|c|c|c|}
\hline S916 & $\mathrm{O}=\mathrm{c} 1 \mathrm{cnc} 2 \mathrm{c}([\mathrm{nH}] 1) \operatorname{ccs} 2$ & -0.12 & -0.85 & 6.50 & 6.74 & 15.14 & 14.62 & 0.52 & 0.67 \\
\hline S917 & $\mathrm{O}=\mathrm{c} 1 \mathrm{nnn} 2 \mathrm{c}([\mathrm{nH}] 1) \sec 2$ & 1.72 & 0.98 & 8.55 & 10.09 & 19.42 & 21.33 & 0.38 & 0.46 \\
\hline S918 & $\mathrm{O}=\mathrm{c} 1 \mathrm{nnc} 2 \mathrm{c}([\mathrm{nH}] 1) \operatorname{ccs} 2$ & 1.83 & 0.98 & 9.93 & 11.21 & 21.63 & 21.71 & 0.72 & 0.84 \\
\hline S919 & $\mathrm{O}=\mathrm{c} 1[\mathrm{nH}] \mathrm{c}(=\mathrm{O}) \mathrm{c} 2 \mathrm{c}([\mathrm{nH}] 1) \mathrm{scc} 2$ & 0.53 & -0.08 & 6.47 & 5.91 & 13.74 & 13.99 & 0.56 & 0.44 \\
\hline S9190 & $\mathrm{O}=\mathrm{c} 1 \mathrm{cc}[\mathrm{nH}] \mathrm{c} 2 \mathrm{c} 1 \mathrm{c} \operatorname{coc} 2=\mathrm{O}$ & 0.76 & 0.99 & 7.86 & 8.32 & 17.03 & 16.75 & 0.55 & 0.48 \\
\hline S9191 & $\mathrm{O}=\mathrm{c} 1 \mathrm{cc}[\mathrm{nH}] \mathrm{c} 2 \mathrm{c} 1 \mathrm{c}(=\mathrm{O}) \mathrm{occ} 2$ & -0.20 & 0.99 & 13.81 & 13.60 & 31.13 & 30.23 & 0.44 & 0.59 \\
\hline S9193 & $\mathrm{O}=\mathrm{c} 1 \mathrm{ccc} 2 \mathrm{c}(\mathrm{o} 1)[\mathrm{nH}] \operatorname{ccc} 2=\mathrm{O}$ & 1.06 & 0.99 & 11.31 & 9.26 & 20.20 & 19.98 & 0.41 & 0.52 \\
\hline S9197 & $\mathrm{O}=\mathrm{c} 1 \mathrm{ccc} 2 \mathrm{c}(\mathrm{o} 1)[\mathrm{nH}] \mathrm{cnc} 2=\mathrm{O}$ & 2.90 & 2.82 & 13.42 & 11.91 & 23.40 & 22.94 & 0.32 & 0.41 \\
\hline S9198 & $\mathrm{O}=\mathrm{c} 1 \mathrm{occc} 2 \mathrm{c} 1 \mathrm{nccn} 2$ & 0.98 & 2.05 & 5.26 & 7.60 & 15.11 & 14.82 & 0.52 & 0.67 \\
\hline S920 & $\mathrm{O}=\mathrm{c} 1[\mathrm{nH}] \mathrm{c}(=\mathrm{O}) \mathrm{c} 2 \mathrm{c}([\mathrm{nH}] 1) \mathrm{ccs} 2$ & 0.54 & -0.08 & 7.68 & 6.67 & 15.84 & 14.31 & 0.32 & 0.35 \\
\hline S9207 & $\mathrm{O}=\mathrm{c} 1 \mathrm{ccc} 2 \mathrm{c}(\mathrm{o} 1) \mathrm{nc}[\mathrm{nH}] \mathrm{c} 2=\mathrm{O}$ & 2.24 & 2.82 & 11.93 & 10.59 & 21.30 & 17.91 & 0.70 & 0.56 \\
\hline S921 & $\mathrm{O}=\mathrm{c} 1[\mathrm{nH}] \mathrm{c} 2 \mathrm{sccc} 2[\mathrm{nH}] \mathrm{c} 1=\mathrm{O}$ & 0.73 & -0.08 & 6.64 & 8.92 & 18.56 & 23.29 & 0.67 & 0.62 \\
\hline S922 & $\mathrm{O}=\mathrm{c} 1 \mathrm{oc}(=\mathrm{O}) \mathrm{c} 2 \mathrm{c}([\mathrm{nH}] 1) \mathrm{scc} 2$ & 2.00 & 1.52 & 8.92 & 9.07 & 17.15 & 17.68 & 0.63 & 0.52 \\
\hline S923 & $\mathrm{O}=\mathrm{c} 1 \mathrm{oc}(=\mathrm{O}) \mathrm{c} 2 \mathrm{c}([\mathrm{nH}] 1) \mathrm{ccs} 2$ & 2.03 & 1.52 & 10.02 & 10.13 & 19.01 & 18.08 & 0.76 & 0.60 \\
\hline S924 & $\mathrm{O}=\mathrm{c} 1[\mathrm{nH}] \mathrm{c} 2 \mathrm{scc} 2 \mathrm{oc} 1=\mathrm{O}$ & 1.70 & 1.52 & 7.52 & 10.03 & 17.21 & 22.89 & 1.04 & 1.10 \\
\hline S925 & $\mathrm{O}=\mathrm{c} 1[\mathrm{nH}] \mathrm{c} 2 \operatorname{css} \mathrm{2} 2 \mathrm{oc} 1=\mathrm{O}$ & 1.74 & 1.52 & 8.40 & 10.84 & 17.98 & 23.11 & 0.42 & 0.57 \\
\hline S9258 & $\mathrm{O}=\mathrm{c} 1 \mathrm{c} \operatorname{coc} 2 \mathrm{c} 1 \mathrm{ccoc} 2=\mathrm{O}$ & 1.88 & 2.59 & 6.14 & 7.76 & 12.63 & 13.98 & 0.48 & 0.41 \\
\hline S9259 & $\mathrm{O}=\mathrm{c} 1 \mathrm{c} \operatorname{coc} 2 \mathrm{c} 1 \mathrm{c}(=\mathrm{O}) \mathrm{occ} 2$ & 1.00 & 2.59 & 8.03 & 9.44 & 21.72 & 21.53 & 0.35 & 0.46 \\
\hline S926 & $\mathrm{O}=\mathrm{c} 1 \mathrm{sc}(=\mathrm{O}) \mathrm{c} 2 \mathrm{c}([\mathrm{nH}] 1) \mathrm{scc} 2$ & 1.07 & 0.83 & 7.57 & 8.05 & 14.19 & 16.19 & 0.27 & 0.39 \\
\hline S927 & $\mathrm{O}=\mathrm{c} 1 \mathrm{sc}(=\mathrm{O}) \mathrm{c} 2 \mathrm{c}([\mathrm{nH}] 1) \mathrm{ccs} 2$ & 1.06 & 0.83 & 9.04 & 9.09 & 16.56 & 16.58 & 0.49 & 0.64 \\
\hline S928 & $\mathrm{O}=\mathrm{c} 1[\mathrm{nH}] \mathrm{c} 2 \mathrm{sccc} 2 \mathrm{sc} 1=\mathrm{O}$ & 1.04 & 0.83 & 6.24 & 9.14 & 15.80 & 21.91 & 0.30 & 0.42 \\
\hline S929 & $\mathrm{O}=\mathrm{c} 1[\mathrm{nH}] \mathrm{c} 2 \operatorname{cssc} 2 \mathrm{sc} 1=\mathrm{O}$ & 1.04 & 0.83 & 7.68 & 9.91 & 17.20 & 22.10 & 0.45 & 0.60 \\
\hline S930 & $\mathrm{O}=\mathrm{c} 1 \mathrm{cc}[\mathrm{nH}] \mathrm{c} 2 \mathrm{c} 1 \mathrm{scc} 2$ & -2.75 & -2.68 & 9.63 & 7.33 & 24.84 & 19.12 & 0.51 & 0.64 \\
\hline S931 & $\mathrm{O}=\mathrm{c} 1 \mathrm{cc}[\mathrm{nH}] \mathrm{c} 2 \mathrm{c} 1 \mathrm{ccs} 2$ & -2.81 & -2.68 & 9.13 & 6.14 & 23.31 & 18.62 & 0.83 & 0.79 \\
\hline S932 & $\mathrm{O}=\mathrm{c} 1 \mathrm{nc}[\mathrm{nH}] \mathrm{c} 2 \mathrm{c} 1 \mathrm{scc} 2$ & -0.84 & -0.85 & 11.61 & 10.78 & 27.24 & 23.96 & 0.32 & 0.45 \\
\hline S933 & $\mathrm{O}=\mathrm{c} 1 \mathrm{nc}[\mathrm{nH}] \mathrm{c} 2 \mathrm{c} 1 \mathrm{ccs} 2$ & -0.95 & -0.85 & 11.15 & 9.39 & 26.03 & 23.38 & 0.48 & 0.63 \\
\hline S934 & $\mathrm{c} 1 \mathrm{cnc} 2 \mathrm{c}(\mathrm{n} 1) \operatorname{scc} 2$ & -2.00 & -1.62 & 2.24 & 3.02 & 6.98 & 9.39 & 0.34 & 0.46 \\
\hline S935 & $\mathrm{c} 1 \mathrm{nnc} 2 \mathrm{c}(\mathrm{n} 1) \mathrm{ccs} 2$ & 0.17 & 0.21 & 5.98 & 5.12 & 13.25 & 11.01 & 0.70 & 0.55 \\
\hline S936 & $\mathrm{c} 1 \mathrm{nnc} 2 \mathrm{c}(\mathrm{n} 1) \operatorname{scc} 2$ & 0.15 & 0.21 & 5.50 & 4.92 & 12.50 & 10.93 & 0.99 & 1.05 \\
\hline S937 & $\mathrm{O}=\mathrm{c} 1[\mathrm{nH}] \mathrm{cnc} 2 \mathrm{c} 1 \mathrm{scc} 2$ & -1.47 & -0.85 & 6.32 & 5.45 & 15.10 & 12.50 & 0.68 & 0.58 \\
\hline S938 & $\mathrm{O}=\mathrm{c} 1[\mathrm{nH}] \mathrm{cnc} 2 \mathrm{c} 1 \mathrm{ccs} 2$ & -1.43 & -0.85 & 6.72 & 5.62 & 14.55 & 12.48 & 0.69 & 0.54 \\
\hline
\end{tabular}




\begin{tabular}{|c|c|c|c|c|c|c|c|c|c|}
\hline S941 & $\mathrm{O}=\mathrm{c} 1 \mathrm{ocn} \operatorname{c} 2 \mathrm{c} 1 \mathrm{scc} 2$ & 0.21 & 0.75 & 4.67 & 5.33 & 12.76 & 11.01 & 0.45 & 0.56 \\
\hline S942 & $\mathrm{O}=\mathrm{c} 1 \mathrm{ocn} \mathrm{c} 2 \mathrm{c} 1 \mathrm{ccs} 2$ & 0.24 & 0.75 & 3.83 & 5.20 & 11.21 & 10.91 & 0.61 & 0.55 \\
\hline S945 & $\mathrm{O}=\mathrm{c} 1 \mathrm{scn} \mathrm{c} 2 \mathrm{c} 1 \mathrm{scc} 2$ & -0.68 & 0.06 & 3.87 & 4.52 & 11.49 & 10.16 & 0.75 & 0.73 \\
\hline S946 & $\mathrm{O}=\mathrm{c} 1 \mathrm{scn} \mathrm{c} 2 \mathrm{c} 1 \mathrm{ccs} 2$ & -0.63 & 0.06 & 3.37 & 4.41 & 10.13 & 10.06 & 0.68 & 0.62 \\
\hline S947 & $\mathrm{n} 1 \mathrm{nnc} 2 \mathrm{c}(\mathrm{n} 1) \mathrm{scc} 2$ & 2.42 & 2.04 & 9.30 & 8.04 & 17.50 & 14.80 & 0.42 & 0.53 \\
\hline S954 & $\mathrm{O}=\mathrm{c} 1[\mathrm{nH}] \mathrm{nnc} 2 \mathrm{c} 1 \mathrm{ccs} 2$ & 0.48 & 0.98 & 4.98 & 6.52 & 10.95 & 11.80 & 0.74 & 0.62 \\
\hline S955 & $\mathrm{O}=\mathrm{c} 1[\mathrm{nH}] \mathrm{nnc} 2 \mathrm{c} 1 \mathrm{scc} 2$ & 0.45 & 0.98 & 5.29 & 6.15 & 11.91 & 11.75 & 0.31 & 0.42 \\
\hline S964 & $\mathrm{O}=\mathrm{c} 1 \mathrm{ccoc} 2 \mathrm{c} 1 \mathrm{scc} 2$ & -1.47 & -1.08 & 4.37 & 4.30 & 15.56 & 12.71 & 0.33 & 0.44 \\
\hline S965 & $\mathrm{O}=\mathrm{c} 1 \mathrm{c} \operatorname{coc} 2 \mathrm{c} 1 \mathrm{ccs} 2$ & -1.47 & -1.08 & 4.56 & 3.92 & 14.86 & 12.43 & 0.61 & 0.70 \\
\hline S966 & $\mathrm{O}=\mathrm{c} 1 \mathrm{n} \operatorname{coc} 2 \mathrm{c} 1 \mathrm{scc} 2$ & 0.39 & 0.75 & 5.95 & 6.86 & 17.60 & 16.30 & 0.63 & 0.51 \\
\hline S967 & $\mathrm{O}=\mathrm{c} 1 \mathrm{n} \operatorname{coc} 2 \mathrm{c} 1 \mathrm{ccs} 2$ & 0.34 & 0.75 & 5.63 & 6.28 & 16.58 & 15.94 & 0.61 & 0.70 \\
\hline S968 & $\mathrm{O}=\mathrm{c} 1 \operatorname{css} 2 \mathrm{c} 1 \operatorname{cs} 2$ & -2.01 & -1.77 & 3.93 & 3.25 & 14.02 & 12.16 & 0.29 & 0.41 \\
\hline S9686 & $\mathrm{n} 1 \mathrm{cnc} 2 \mathrm{c}(\mathrm{c} 1) \mathrm{c}[\mathrm{nH}] \mathrm{c} 2$ & -3.16 & -2.53 & 8.39 & 5.71 & 18.06 & 15.15 & 0.45 & 0.60 \\
\hline S9687 & $\mathrm{n} 1 \mathrm{cnc} 2 \mathrm{c}(\mathrm{c} 1) \operatorname{coc} 2$ & -1.04 & -0.93 & 4.69 & 3.90 & 10.64 & 10.73 & 0.36 & 0.47 \\
\hline S9688 & $\mathrm{n} 1 \mathrm{cnc} 2 \mathrm{c}(\mathrm{c} 1) \mathrm{csc} 2$ & -1.55 & -1.62 & 4.29 & 3.20 & 10.70 & 10.24 & 0.70 & 0.56 \\
\hline S9689 & $\mathrm{c} 1 \mathrm{ncc} 2 \mathrm{c}(\mathrm{n} 1) \mathrm{c}[\mathrm{nH}] \mathrm{n} 2$ & -1.52 & -0.70 & 8.07 & 6.02 & 14.59 & 13.39 & 0.38 & 0.37 \\
\hline S969 & $\mathrm{O}=\mathrm{c} 1 \operatorname{cssc} 2 \mathrm{c} 1 \mathrm{scc} 2$ & -1.98 & -1.77 & 3.69 & 3.68 & 14.71 & 12.46 & 0.65 & 0.51 \\
\hline S9690 & $\mathrm{c} 1 \mathrm{ncc} 2 \mathrm{c}(\mathrm{n} 1) \mathrm{n}[\mathrm{nH}] \mathrm{c} 2$ & -1.28 & -0.70 & 8.86 & 7.19 & 18.99 & 15.95 & 1.00 & 0.82 \\
\hline S9692 & c1ncc2c(n1)noc2 & 1.19 & 0.90 & 8.87 & 6.33 & 16.81 & 12.86 & 0.71 & 0.58 \\
\hline S9694 & $\mathrm{c} 1 \mathrm{ncc} 2 \mathrm{c}(\mathrm{n} 1) \mathrm{nsc} 2$ & 0.30 & 0.21 & 7.78 & 5.57 & 15.92 & 12.21 & 0.43 & 0.59 \\
\hline S9695 & $\mathrm{n} 1 \mathrm{cnc} 2 \mathrm{c}(\mathrm{c} 1) \mathrm{n}[\mathrm{nH}] \mathrm{n} 2$ & 0.38 & 1.13 & 7.11 & 6.48 & 13.13 & 11.94 & 0.41 & 0.53 \\
\hline S9696 & $\mathrm{n} 1 \mathrm{cnc} 2 \mathrm{c}(\mathrm{c} 1)$ non 2 & 3.14 & 2.73 & 7.80 & 6.58 & 11.58 & 10.19 & 0.24 & 0.36 \\
\hline S9697 & $\mathrm{n} 1 \mathrm{cnc} 2 \mathrm{c}(\mathrm{c} 1) \mathrm{nsn} 2$ & 1.89 & 2.04 & 5.08 & 5.76 & 9.53 & 9.37 & 0.61 & 0.54 \\
\hline S97 & $\mathrm{O}=\mathrm{c} 1 \mathrm{ccocc} 1$ & -0.58 & -0.65 & 4.94 & 4.06 & 15.76 & 13.46 & 0.52 & 0.40 \\
\hline S970 & $\mathrm{O}=\mathrm{c} 1 \mathrm{cn}[\mathrm{nH}] \mathrm{c} 2 \mathrm{c} 1 \mathrm{scc} 2$ & -0.92 & -0.85 & 8.25 & 7.01 & 20.28 & 16.12 & 0.73 & 0.81 \\
\hline S9704 & $\mathrm{c} 1 \mathrm{ncc} 2 \mathrm{c}(\mathrm{n} 1) \mathrm{ncnc} 2$ & 1.76 & 1.51 & 7.55 & 6.21 & 13.33 & 11.62 & 0.71 & 0.53 \\
\hline S9705 & $\mathrm{n} 1 \mathrm{cnc} 2 \mathrm{c}(\mathrm{c} 1) \mathrm{ncnc} 2$ & 1.49 & 1.51 & 4.03 & 5.47 & 5.43 & 9.99 & 0.68 & 0.61 \\
\hline S9706 & $\mathrm{n} 1 \mathrm{cnc} 2 \mathrm{c}(\mathrm{c} 1) \mathrm{cnnn} 2$ & 3.99 & 3.34 & 11.33 & 9.69 & 18.44 & 16.37 & 0.47 & 0.62 \\
\hline S9707 & $\mathrm{n} 1 \mathrm{cnc} 2 \mathrm{c}(\mathrm{c} 1) \mathrm{nnnc} 2$ & 3.68 & 3.34 & 8.36 & 8.50 & 14.26 & 13.75 & 0.29 & 0.41 \\
\hline S9708 & $\mathrm{O}=\mathrm{c} 1[\mathrm{nH}] \mathrm{ncc} 2 \mathrm{c} 1 \mathrm{ncnc} 2$ & 1.99 & 2.28 & 7.91 & 7.19 & 17.06 & 13.31 & 0.62 & 0.55 \\
\hline S9709 & $\mathrm{O}=\mathrm{c} 1[\mathrm{nH}] \mathrm{ncc} 2 \mathrm{c} 1 \mathrm{cncn} 2$ & 2.04 & 2.28 & 5.53 & 6.83 & 10.49 & 11.41 & 0.76 & 0.69 \\
\hline
\end{tabular}




\begin{tabular}{|c|c|c|c|c|c|c|c|c|c|}
\hline S971 & $\mathrm{O}=\mathrm{c} 1 \mathrm{cn}[\mathrm{nH}] \mathrm{c} 2 \mathrm{c} 1 \mathrm{ccs} 2$ & -0.98 & -0.85 & 7.33 & 6.01 & 18.37 & 15.70 & 0.62 & 0.60 \\
\hline S9712 & $\mathrm{O}=\mathrm{c} 1 \mathrm{ncc} 2 \mathrm{c}([\mathrm{nH}] 1) \mathrm{ncnc} 2$ & 3.30 & 2.28 & 8.61 & 8.56 & 17.71 & 16.29 & 0.84 & 0.73 \\
\hline S9713 & $\mathrm{O}=\mathrm{c} 1 \mathrm{ncc} 2 \mathrm{c}([\mathrm{nH}] 1) \mathrm{cncn} 2$ & 3.40 & 2.28 & 9.88 & 9.85 & 18.63 & 17.96 & 0.53 & 0.70 \\
\hline S9732 & $\mathrm{O}=\mathrm{c} 1 \mathrm{cnc} 2 \mathrm{c}([\mathrm{nH}] 1) \mathrm{cncn} 2$ & 3.00 & 2.28 & 9.91 & 9.55 & 14.20 & 15.92 & 0.69 & 0.83 \\
\hline S9733 & $\mathrm{O}=\mathrm{c} 1 \mathrm{cnc} 2 \mathrm{c}([\mathrm{nH}] 1) \mathrm{ncnc} 2$ & 2.66 & 2.28 & 6.91 & 7.52 & 11.44 & 12.63 & 0.53 & 0.71 \\
\hline S9734 & $\mathrm{O}=\mathrm{c} 1 \mathrm{nnc} 2 \mathrm{c}([\mathrm{nH}] 1) \mathrm{cncn} 2$ & 4.84 & 4.11 & 13.28 & 13.80 & 19.66 & 22.59 & 0.70 & 0.52 \\
\hline S9735 & $\mathrm{O}=\mathrm{c} 1 \mathrm{nnc} 2 \mathrm{c}([\mathrm{nH}] 1) \mathrm{ncnc} 2$ & 4.49 & 4.11 & 10.16 & 11.32 & 16.81 & 18.31 & 0.41 & 0.54 \\
\hline S9736 & $\mathrm{O}=\mathrm{c} 1[\mathrm{nH}] \mathrm{c} 2 \mathrm{cncnc} 2 \mathrm{c}(=\mathrm{O})[\mathrm{nH}] 1$ & 3.46 & 3.05 & 10.85 & 9.78 & 18.04 & 16.60 & 0.73 & 0.65 \\
\hline S9737 & $\mathrm{O}=\mathrm{c} 1[\mathrm{nH}] \mathrm{c} 2 \mathrm{ncncc} 2 \mathrm{c}(=\mathrm{O})[\mathrm{nH}] 1$ & 3.44 & 3.05 & 8.55 & 8.14 & 11.89 & 13.03 & 0.62 & 0.56 \\
\hline S9738 & $\mathrm{O}=\mathrm{c} 1[\mathrm{nH}] \mathrm{c} 2 \mathrm{ncncc} 2[\mathrm{nH}] \mathrm{c} 1=\mathrm{O}$ & 3.80 & 3.05 & 9.94 & 10.43 & 17.61 & 21.05 & 0.72 & 0.68 \\
\hline S9740 & $\mathrm{O}=\mathrm{c} 1 \mathrm{oc}(=\mathrm{O}) \mathrm{c} 2 \mathrm{c}([\mathrm{nH}] 1) \mathrm{ncnc} 2$ & 4.93 & 4.65 & 11.22 & 10.57 & 15.91 & 15.45 & 0.28 & 0.40 \\
\hline S9743 & $\mathrm{O}=\mathrm{c} 1 \mathrm{sc}(=\mathrm{O}) \mathrm{c} 2 \mathrm{c}([\mathrm{nH}] 1) \mathrm{cncn} 2$ & 3.90 & 3.96 & 11.88 & 11.88 & 17.89 & 18.52 & 0.50 & 0.63 \\
\hline S9744 & $\mathrm{O}=\mathrm{c} 1 \mathrm{sc}(=\mathrm{O}) \mathrm{c} 2 \mathrm{c}([\mathrm{nH}] 1) \mathrm{ncnc} 2$ & 3.88 & 3.96 & 9.35 & 9.60 & 12.91 & 14.12 & 0.76 & 0.61 \\
\hline S9746 & $\mathrm{O}=\mathrm{c} 1[\mathrm{nH}] \mathrm{c} 2 \mathrm{ncnc} 2 \mathrm{sc} 1=\mathrm{O}$ & 3.94 & 3.96 & 9.47 & 10.43 & 15.37 & 19.04 & 0.59 & 0.82 \\
\hline S9747 & $\mathrm{O}=\mathrm{c} 1 \mathrm{cc}[\mathrm{nH}] \mathrm{c} 2 \mathrm{c} 1 \mathrm{cncn} 2$ & 0.19 & 0.45 & 9.41 & 8.08 & 19.05 & 17.21 & 0.76 & 0.68 \\
\hline S9748 & $\mathrm{O}=\mathrm{c} 1 \mathrm{cc}[\mathrm{nH}] \mathrm{c} 2 \mathrm{c} 1 \mathrm{ncnc} 2$ & 0.20 & 0.45 & 12.70 & 10.72 & 26.58 & 22.94 & 0.52 & 0.65 \\
\hline S9749 & $\mathrm{O}=\mathrm{c} 1 \mathrm{nc}[\mathrm{nH}] \mathrm{c} 2 \mathrm{c} 1 \mathrm{cncn} 2$ & 2.07 & 2.28 & 11.40 & 10.85 & 21.89 & 20.64 & 0.86 & 0.71 \\
\hline S9750 & $\mathrm{O}=\mathrm{c} 1 \mathrm{nc}[\mathrm{nH}] \mathrm{c} 2 \mathrm{c} 1 \mathrm{ncnc} 2$ & 2.11 & 2.28 & 14.87 & 13.94 & 28.50 & 27.36 & 0.37 & 0.50 \\
\hline S9751 & $\mathrm{c} 1 \mathrm{ncc} 2 \mathrm{c}(\mathrm{n} 1) \mathrm{nccn} 2$ & 0.99 & 1.51 & 6.18 & 6.03 & 12.12 & 11.23 & 0.70 & 0.56 \\
\hline S9752 & c1ncc $2 \mathrm{c}(\mathrm{n} 1) \mathrm{ncnn} 2$ & 3.07 & 3.34 & 7.03 & 7.45 & 9.26 & 11.44 & 0.57 & 0.48 \\
\hline S9753 & $\mathrm{n} 1 \mathrm{cnc} 2 \mathrm{c}(\mathrm{c} 1) \mathrm{ncnn} 2$ & 3.09 & 3.34 & 7.42 & 7.90 & 12.44 & 12.42 & 0.70 & 0.60 \\
\hline S9754 & $\mathrm{O}=\mathrm{c} 1[\mathrm{nH}] \mathrm{cnc} 2 \mathrm{c} 1 \mathrm{cncn} 2$ & 1.62 & 2.28 & 10.47 & 9.15 & 16.01 & 15.05 & 0.41 & 0.51 \\
\hline S9755 & $\mathrm{O}=\mathrm{c} 1[\mathrm{nH}] \mathrm{cnc} 2 \mathrm{c} 1 \mathrm{ncnc} 2$ & 1.33 & 2.28 & 9.96 & 8.77 & 18.15 & 15.33 & 0.77 & 0.66 \\
\hline S9758 & $\mathrm{O}=\mathrm{c} 1 \mathrm{ocnc} 2 \mathrm{c} 1 \mathrm{cncn} 2$ & 3.33 & 3.88 & 8.42 & 8.00 & 11.55 & 12.21 & 0.94 & 0.87 \\
\hline S9759 & $\mathrm{O}=\mathrm{c} 1 \mathrm{ocnc} 2 \mathrm{c} 1 \mathrm{ncnc} 2$ & 3.03 & 3.88 & 8.17 & 8.30 & 14.79 & 13.43 & 0.61 & 0.80 \\
\hline S976 & $\mathrm{O}=\mathrm{c} 1 \mathrm{n} \csc 2 \mathrm{c} 1 \mathrm{scc} 2$ & -0.29 & 0.06 & 5.70 & 6.30 & 17.50 & 16.21 & 0.32 & 0.45 \\
\hline S977 & $\mathrm{O}=\mathrm{c} 1 \mathrm{n} \csc 2 \mathrm{c} 1 \mathrm{ccs} 2$ & -0.35 & 0.06 & 4.56 & 5.67 & 15.69 & 15.82 & 0.33 & 0.47 \\
\hline S9771 & $\mathrm{O}=\mathrm{c} 1[\mathrm{nH}] \mathrm{nnc} 2 \mathrm{c} 1 \mathrm{ncnc} 2$ & 3.22 & 4.11 & 7.73 & 8.99 & 13.62 & 13.25 & 0.31 & 0.43 \\
\hline S978 & $\mathrm{O}=\mathrm{c} 1 \mathrm{nn}[\mathrm{nH}] \mathrm{c} 2 \mathrm{c} 1 \mathrm{scc} 2$ & 0.95 & 0.98 & 10.79 & 11.48 & 23.97 & 23.21 & 0.57 & 0.43 \\
\hline S9781 & $\mathrm{O}=\mathrm{c} 1 \mathrm{ccoc} 2 \mathrm{c} 1 \mathrm{cncn} 2$ & 1.64 & 2.05 & 6.61 & 6.61 & 12.69 & 12.61 & 0.86 & 0.68 \\
\hline S9782 & $\mathrm{O}=\mathrm{c} 1 \mathrm{ccoc} 2 \mathrm{c} 1 \mathrm{ncnc} 2$ & 1.40 & 2.05 & 7.79 & 7.45 & 18.00 & 15.82 & 0.58 & 0.43 \\
\hline
\end{tabular}




\begin{tabular}{|c|c|c|c|c|c|c|c|c|c|}
\hline S9783 & $\mathrm{O}=\mathrm{c} 1 \mathrm{n} \operatorname{coc} 2 \mathrm{c} 1 \mathrm{cncn} 2$ & 3.49 & 3.88 & 8.96 & 8.49 & 15.78 & 14.79 & 0.62 & 0.89 \\
\hline S9784 & $\mathrm{O}=\mathrm{c} 1 \mathrm{ncoc} 2 \mathrm{c} 1 \mathrm{ncnc} 2$ & 3.28 & 3.88 & 10.36 & 9.78 & 20.21 & 18.99 & 0.52 & 0.67 \\
\hline S9787 & $\mathrm{O}=\mathrm{c} 1 \mathrm{cn}[\mathrm{nH}] \mathrm{c} 2 \mathrm{c} 1 \mathrm{cncn} 2$ & 2.03 & 2.28 & 6.33 & 7.73 & 12.60 & 13.87 & 0.81 & 0.93 \\
\hline S979 & $\mathrm{O}=\mathrm{c} 1 \mathrm{nn}[\mathrm{nH}] \mathrm{c} 2 \mathrm{c} 1 \mathrm{ccs} 2$ & 0.85 & 0.98 & 9.89 & 10.29 & 22.31 & 22.71 & 0.51 & 0.66 \\
\hline S9795 & $\mathrm{O}=\mathrm{c} 1 \mathrm{nn}[\mathrm{nH}] \mathrm{c} 2 \mathrm{c} 1 \mathrm{cncn} 2$ & 3.88 & 4.11 & 9.74 & 11.52 & 17.42 & 19.55 & 0.71 & 0.67 \\
\hline S98 & $\mathrm{O}=\mathrm{c} 1 \mathrm{ccocn} 1$ & 1.24 & 1.18 & 7.88 & 6.57 & 19.00 & 17.03 & 0.65 & 0.55 \\
\hline S9802 & $\mathrm{O}=\mathrm{c} 1 \mathrm{loc} 2 \mathrm{ncncc} 2 \mathrm{c}(=\mathrm{O})[\mathrm{nH}] 1$ & 4.68 & 4.65 & 10.72 & 9.36 & 14.61 & 12.44 & 0.63 & 0.79 \\
\hline S9803 & $\mathrm{O}=\mathrm{c} 1 \mathrm{oc} 2 \mathrm{cncnc} 2 \mathrm{c}(=\mathrm{O})[\mathrm{nH}] 1$ & 4.47 & 4.65 & 10.75 & 9.21 & 15.27 & 13.49 & 0.43 & 0.62 \\
\hline S9805 & $\mathrm{O}=\mathrm{c} 1 \mathrm{sc} 2 \mathrm{cncnc} 2 \mathrm{c}(=\mathrm{O})[\mathrm{nH}] 1$ & 3.63 & 3.96 & 8.68 & 8.43 & 13.91 & 12.84 & 0.38 & 0.50 \\
\hline S9816 & $\mathrm{O}=\mathrm{c} 1 \mathrm{sc} 2 \mathrm{c}(\mathrm{s} 1) \mathrm{cncn} 2$ & 2.17 & 1.89 & 5.76 & 6.10 & 10.00 & 11.02 & 0.73 & 0.57 \\
\hline S9822 & $\mathrm{n} 1 \mathrm{nnnc} 2 \mathrm{c}(\mathrm{c} 1) \mathrm{c}[\mathrm{nH}] \mathrm{c} 2$ & -0.93 & -0.70 & 11.81 & 9.97 & 25.88 & 21.22 & 0.78 & 0.71 \\
\hline $\mathbf{S 9 8 2 3}$ & $\mathrm{n} 1 \mathrm{nnc} 2 \mathrm{c}(\mathrm{c} 1) \operatorname{coc} 2$ & 1.11 & 0.90 & 7.68 & 7.35 & 17.68 & 15.67 & 0.91 & 0.74 \\
\hline S9824 & $\mathrm{n} 1 \mathrm{nnc} 2 \mathrm{c}(\mathrm{c} 1) \mathrm{csc} 2$ & 0.63 & 0.21 & 7.40 & 6.71 & 18.22 & 15.31 & 0.70 & 0.55 \\
\hline S9826 & $\mathrm{n} 1 \mathrm{nnc} 2 \mathrm{c}(\mathrm{c} 1) \mathrm{c}[\mathrm{nH}] \mathrm{n} 2$ & 0.95 & 1.13 & 12.40 & 11.61 & 24.44 & 22.37 & 0.69 & 0.49 \\
\hline S9828 & $\mathrm{n} 1 \mathrm{nnc} 2 \mathrm{c}(\mathrm{c} 1) \operatorname{con} 2$ & 3.30 & 2.73 & 12.20 & 9.95 & 19.67 & 18.15 & 0.29 & 0.48 \\
\hline S9830 & $\mathrm{n} 1 \mathrm{nnn} 2 \mathrm{c}(\mathrm{c} 1) \operatorname{csn} 2$ & 2.49 & 2.04 & 11.16 & 9.24 & 20.79 & 17.63 & 0.74 & 0.74 \\
\hline S9831 & $\mathrm{n} 1 \mathrm{nnc} 2 \mathrm{c}(\mathrm{c} 1) \mathrm{n}[\mathrm{nH}] \mathrm{n} 2$ & 2.57 & 2.96 & 10.80 & 10.39 & 19.40 & 17.24 & 0.25 & 0.36 \\
\hline S985 & $\mathrm{O}=\mathrm{c} 1 \mathrm{oc} 2 \operatorname{cssc} 2 \mathrm{c}(=\mathrm{O})[\mathrm{nH}] 1$ & 1.63 & 1.52 & 6.47 & 6.33 & 12.59 & 11.91 & 0.40 & 0.58 \\
\hline S986 & $\mathrm{O}=\mathrm{c} 1 \mathrm{oc} 2 \operatorname{secc} 2 \mathrm{c}(=\mathrm{O})[\mathrm{nH}] 1$ & 1.65 & 1.52 & 5.37 & 6.38 & 11.01 & 11.81 & 0.52 & 0.75 \\
\hline S9867 & $\mathrm{O}=\mathrm{c} 1 \mathrm{cnc} 2 \mathrm{c}([\mathrm{nH}] 1) \mathrm{nnnc} 2$ & 4.88 & 4.11 & 11.28 & 10.10 & 16.75 & 15.46 & 0.97 & 0.77 \\
\hline S9869 & $\mathrm{O}=\mathrm{c} 1 \mathrm{nn} \mathrm{c} 2 \mathrm{c}([\mathrm{nH}] 1) \mathrm{nnnc} 2$ & 6.63 & 5.94 & 14.12 & 13.33 & 17.41 & 19.90 & 0.76 & 0.70 \\
\hline S987 & $\mathrm{O}=\mathrm{c} 1 \mathrm{sc} 2 \operatorname{ccsc} 2 \mathrm{c}(=\mathrm{O})[\mathrm{nH}] 1$ & 0.83 & 0.83 & 6.16 & 5.54 & 12.08 & 11.17 & 0.59 & 0.79 \\
\hline S9871 & $\mathrm{O}=\mathrm{c} 1[\mathrm{nH}] \mathrm{c} 2 \mathrm{nnncc} 2 \mathrm{c}(=\mathrm{O})[\mathrm{nH}] 1$ & 5.71 & 4.88 & 12.86 & 11.31 & 16.97 & 16.55 & 0.38 & 0.56 \\
\hline S9878 & $\mathrm{O}=\mathrm{c} 1 \mathrm{sc}(=\mathrm{O}) \mathrm{c} 2 \mathrm{c}([\mathrm{nH}] 1) \mathrm{nnnc} 2$ & 6.10 & 5.79 & 13.38 & 11.98 & 16.17 & 16.59 & 0.82 & 0.80 \\
\hline S988 & $\mathrm{O}=\mathrm{c} 1 \mathrm{sc} 2 \operatorname{scc} 2 \mathrm{c}(=\mathrm{O})[\mathrm{nH}] 1$ & 0.85 & 0.83 & 5.10 & 5.54 & 10.56 & 11.04 & 0.77 & 0.80 \\
\hline S9885 & c1cnc2c(n1)nnnc2 & 3.28 & 3.34 & 10.35 & 9.74 & 18.87 & 16.47 & 0.74 & 0.56 \\
\hline S989 & $\mathrm{O}=\mathrm{c} 1 \mathrm{oc} 2 \mathrm{c}(\mathrm{o} 1) \operatorname{ccs} 2$ & 0.88 & 0.14 & 5.39 & 5.53 & 13.53 & 13.37 & 0.79 & 0.76 \\
\hline S99 & $\mathrm{O}=\mathrm{c} 1 \mathrm{n} \operatorname{cocn} 1$ & 3.05 & 3.01 & 10.76 & 8.49 & 21.30 & 19.31 & 0.30 & 0.51 \\
\hline S992 & $\mathrm{O}=\mathrm{c} 1 \mathrm{oc} 2 \mathrm{scc} 2 \mathrm{oc} 1=\mathrm{O}$ & 2.74 & 3.12 & 7.68 & 9.62 & 14.35 & 20.63 & 0.88 & 0.62 \\
\hline S993 & $\mathrm{O}=\mathrm{c} 1 \mathrm{oc}(=\mathrm{O}) \mathrm{c} 2 \mathrm{c}(\mathrm{o} 1) \mathrm{ccs} 2$ & 3.13 & 3.12 & 8.95 & 8.44 & 16.05 & 14.48 & 0.79 & 0.65 \\
\hline S994 & $\mathrm{O}=\mathrm{c} 1 \mathrm{oc}(=\mathrm{O}) \mathrm{c} 2 \mathrm{c}(\mathrm{o} 1) \mathrm{scc} 2$ & 3.15 & 3.12 & 7.80 & 8.20 & 14.37 & 14.30 & 0.93 & 0.95 \\
\hline
\end{tabular}


